Florida International University FIU Digital Commons

$2-21-2013$

\title{
Selective and Specific Activation of Rab5 during Endocytosis of Receptor Tyrosine Kinases
}

Ivan Jozic

Florida International University, ijozi001@fiu.edu

DOI: $10.25148 /$ etd.FI13040302

Follow this and additional works at: https://digitalcommons.fiu.edu/etd

\section{Recommended Citation}

Jozic, Ivan, "Selective and Specific Activation of Rab5 during Endocytosis of Receptor Tyrosine Kinases" (2013). FIU Electronic Theses and Dissertations. 813.

https://digitalcommons.fiu.edu/etd/813 


\title{
FLORIDA INTERNATIONAL UNIVERSITY
}

Miami, Florida

\section{SELECTIVE AND SPECIFIC ACTIVATION OF RAB5 DURING ENDOCYTOSIS OF RECEPTOR TYROSINE KINASES}

\author{
A dissertation submitted in partial fulfillment of \\ the requirements for the degree of \\ DOCTOR OF PHILOSOPHY \\ in \\ BIOLOGY
}

by

Ivan Jozic

2013 
To: Dean Kenneth G. Furton

College of Arts and Sciences

This dissertation, written by Ivan Jozic, and entitled Selective and Specific Activation of Rab5 during Endocytosis of Epidermal Growth Factor Receptor, having been approved in respect to style and intellectual content, is referred to you for judgment.

We have read this dissertation and recommend that it be approved.

$\begin{array}{r}\text { Lou Kim } \\ \hline \text { Lidia Kos } \\ \hline \text { John Makemson } \\ \hline \text { Helen Tempest } \\ \hline \text { M. Alejandro Barbieri, Major Professor }\end{array}$

Date of defense: February 21, 2013

The dissertation of Ivan Jozic is approved.

$\begin{array}{r}\begin{array}{r}\text { Dean Kenneth G. Furton } \\ \text { College of Arts and Sciences }\end{array} \\ \hline \begin{array}{r}\text { Dean Lakshmi Reddi } \\ \text { University Graduate School }\end{array}\end{array}$

Florida International University, 2013 
C Copyright 2013 by Ivan Jozic

All rights reserved. 


\section{DEDICATION}

I want to dedicate this dissertation to my family; to my parents for believing in me and making this dream come true; to my brother Dejan who stood by me through thick and thin and who provided me with the emotional support to achieve this goal.

Thank you all for believing in me and being part of this astonishing dream. 


\section{ACKNOWLEDGMENTS}

First and foremost I wish to thank my mentor Dr. M. Alejandro Barbieri for first providing me with a wonderful opportunity and then allowing me the autonomy to independently develop and execute this project. Next, I would like to thank all my committee members: Dr. John Makemson, Dr. Lou Kim, Dr. Helen Tempest and Dr. Lidia Kos, for all their help and all their insightful remarks throughout this journey. I also wish to thank all the past and present members of the Barbieri lab: Monica Rodriguez Silva, Alvaro E. Velandia, Rudolph Samar Saliba, Ana Meneses, Adriana Galvis, Travis Murphy, Hugo Giambini and Alejandro Conde, all of whom helped me at the various stages of this project; without your patience, support and understanding, none of this work would have been possible. 
ABSTRACT OF THE DISSERTATION

\title{
SELECTIVE AND SPECIFIC ACTIVATION OF RAB5 DURING ENDOCYTOSIS OF \\ RECEPTOR TYROSINE KINASES
}

\author{
by
}

Ivan Jozic

Florida Inernational University, 2013

Miami, Florida

\section{Professor M. Alejandro Barbieri, Major Professor}

The Rab family of proteins are low molecular weight GTPases that have the ability to switch between GTP- (active) and GDP- (inactive) bound form, and in that sense act as molecular switches. Through distinct localization on various vesicles and organelles and by cycling through GTP/GDP bound forms, Rabs are able to recruit and activate numerous effector proteins, both spatially and temporally, and hence behave as key regulators of trafficking in both endocytic and biosynhtetic pathways. The Rab5 protein has been shown to regulate transport from plasma membrane to the early endosome as well as activate signaling pathways from the early endosome. This dissertation focused on understanding Rab5 activation via endocytosis of receptor tyrosine kinases (RTKs). First, tyrosine kinase activity of RTKs was linked to endosome fusion by demonstrating that tyrosine kinase inhibitors block endosome fusion and activation of Rab5, and a constitutively active form of Rab5 is able to rescue endosome fusion. However, depending on how much ligand is available at the cell surface, the receptor-ligand complexes can be internalized via a number of distinct pathways. Similarly, Rab5 was activated in a ligand-dependent concentration dependent manner via clathrin- and 
caveolin-mediated pathways, as well as a pathway independent of both. However, overexpression Rabex-5, a nucleotide exchange factor for Rab5, is able to rescue activation even when all of the pathways of EGF-receptor internalization were blocked. Next, the three naturally occurring splice variants of Rabex- 5 selectively activated Rab5. Lastly, Rabex-5 inhibits differentiation of 3T3-L1 and PC12 cells through 1) degradation of signaling endosome via Rab5-dependent fusion with the early endosome, 2) and inhibition of signaling cascade via ubiquitination of Ras through the $\mathrm{ZnF}$ domain at the N-terminus of Rabex-5.

In conclusion, these data shed light on complexity of the endosomal trafficking system where tyrosine kinase activity of the receptor is able to affect endosome fusion; how different endocytic pathways affect activation of one of the key regulators of early endocytic events; and how selective activation of Rab5 via Rabex-5 can control adipogenesis and neurogenesis. 


\section{TABLE OF CONTENTS}

CHAPTER

PAGE

1. Introduction. 1

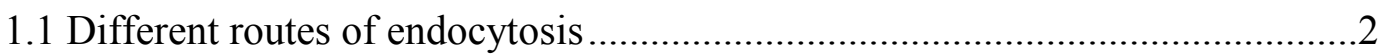

1.2 Abnormalities associated with derailed endocytosis .......................................6

1.3 Endocytosis of Epidermal Growth Factor Receptor (EGFR) ……....................

1.4 Disorders arising from misregulation of EGFR ...............................................10

1.5 Role of small GTPases in intracellular trafficking ........................................11

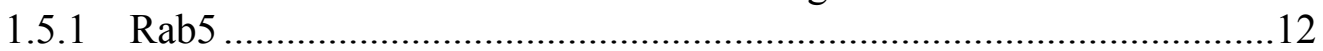

1.5.2 Guanine Nucleotide Exchange Factors (GEFs) ....................................13

1.5.2.1 Ras interaction/interference 1 ................................................14

1.5.2.2 Rabaptin-5 associated exchange factor for Rab5 .....................14

1.6 Disorders associated with aberrant activation of Rab5 …...............................17

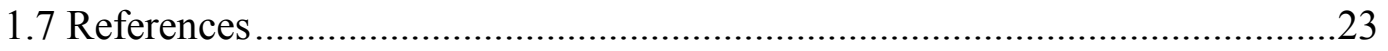

2. Tyrosine kinase activity of receptor tyrosine kinases is required for endosome fusion

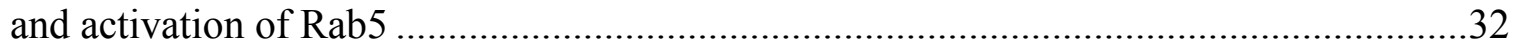

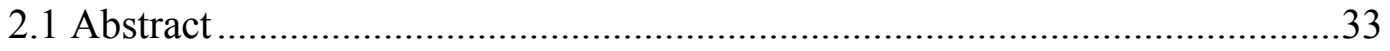

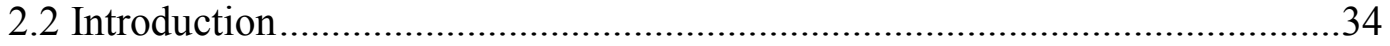

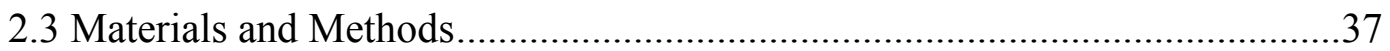

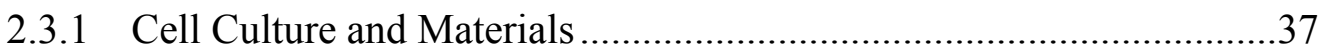

2.3.2 Endocytic Probes and Fractionation .......................................................37

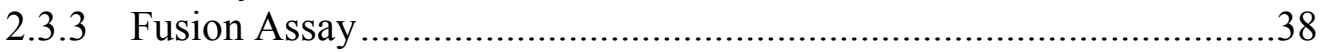

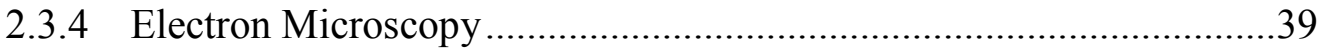

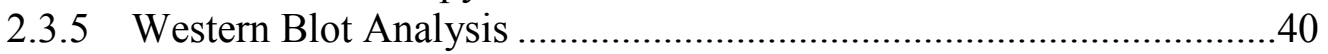

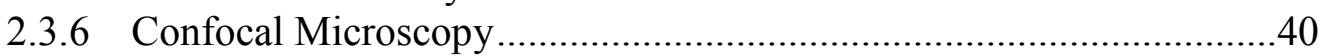

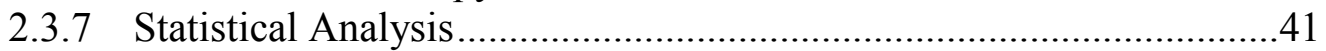

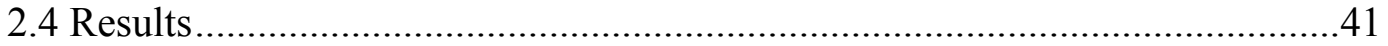

2.4.1 Insulin receptor tyrosine kinase inhibitor blocks in vitro endosome

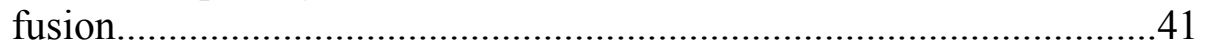

2.4.2 Insulin receptor inhibitor block fusion between endosomes in intact

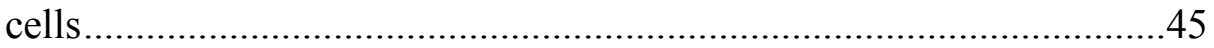

2.4.3 HNMPA-(AM) $)_{3}$ inhibitor bocks Rab5 activation and targeting of Rin1 to membrane upon insulin stimulation.....................................................4

2.4.4 EGF-receptor tyrosine kinase inhibitor blocks fusion between endosomes in intact cells ..................................................................4

2.4.5 AG1478 inhibitor blocks the activation of Rab5 in intact cells..............51

2.4.6 AG1478 inhibitor blocks the recruitment of Rin1 in intact cells ...........51

2.4.7 EGF-receptor tyrosine kinase inhibitor inhibits in vitro endosome

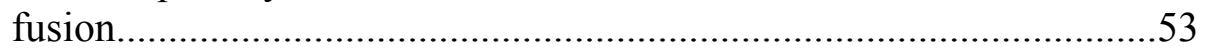

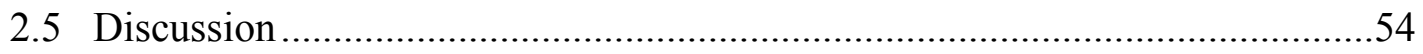

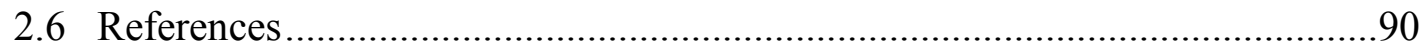


3. Rab5 can be activated through three distinct endocytic routes ...............................95

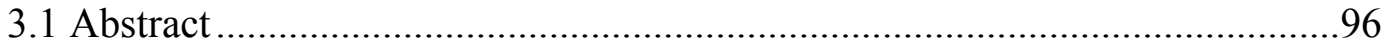

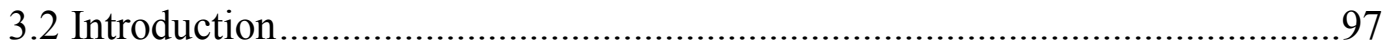

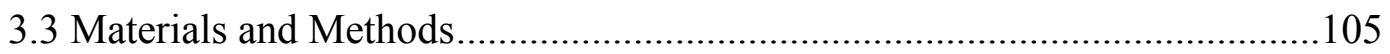

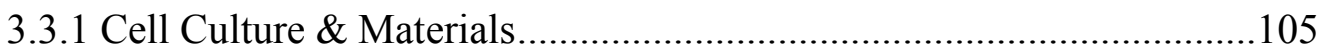

3.3.2 Assessment of Rab5 activity via EEA1 pulldown assay .....................105

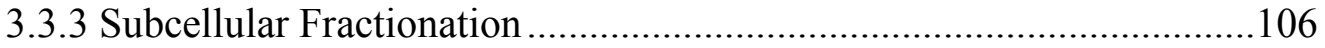

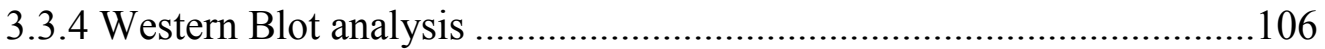

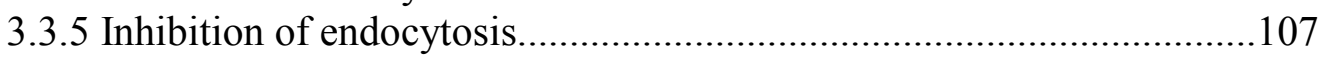

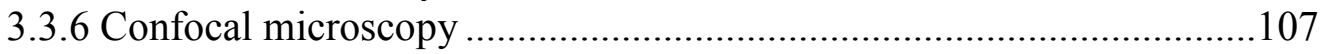

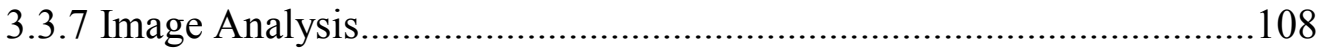

3.3.8 siRNA knockdown of Caveolin, Dynamin, Rin1 and Rabex-5 ...........108

3.4 Results....................................................................................... 108

3.4.1 Activation of Rab5 is dependent on temperature, concentration of ligand

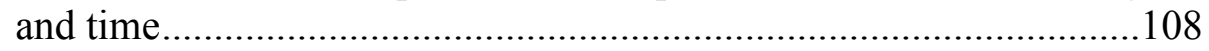

3.4.2 Rab5 can be activated via at least three distinct pathways of EGFR endocytosis...............................................................................110

3.4.3 Rabex-5 and Rin1 can both rescue activation of Rab5 when one of these pathways of EGFR internalization is blocked ...............................112

3.4.4 Rabex-5 splice variants selectively activate Rab5 ..........................113

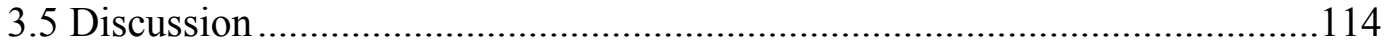

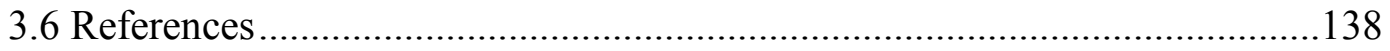

4. The Effect of Rabex-5 on several biological processes .......................................142

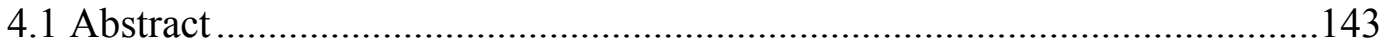

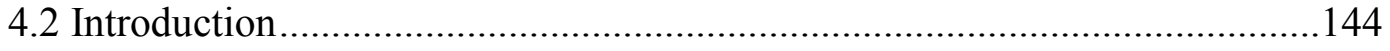

4.3 Materials and Methods........................................................................ 150

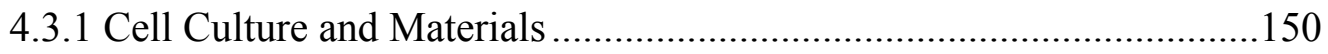

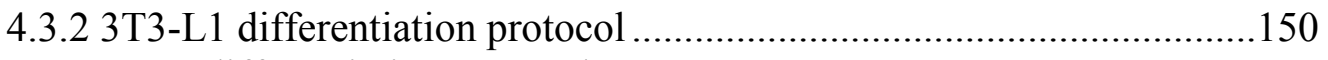

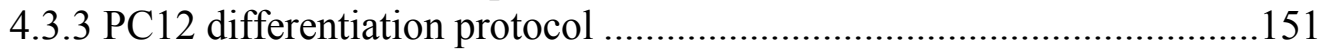

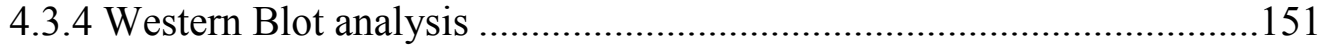

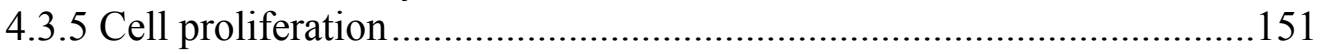

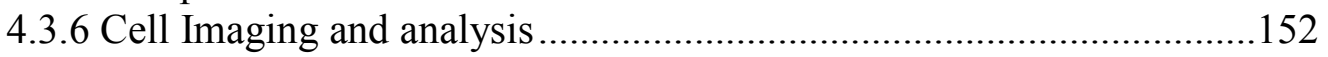

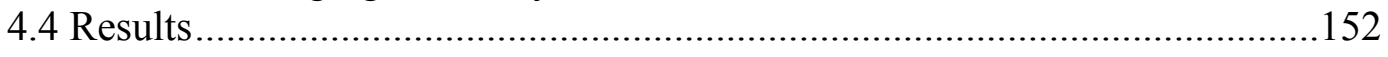

4.4.1 Rabex-5 splice variants selectively inhibit adipogenesis through activation of Rab5 and ubiquitination of Ras. ..........................................152

4.4.2 Rabex-5 splice variants selectively inhibit neurite outgrowth in rat PC12

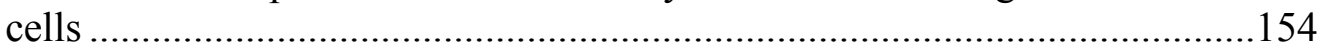

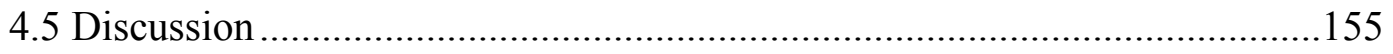

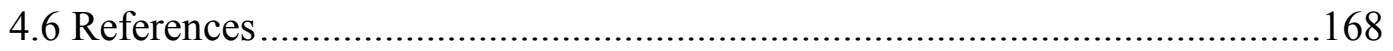

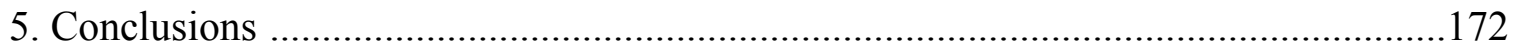

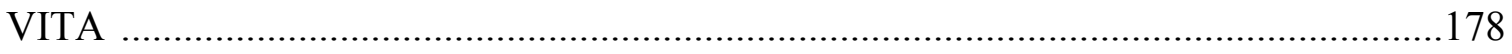


FIGURE

Figure 1. Pathways of EGF-Receptor Endocytosis.........................................................19

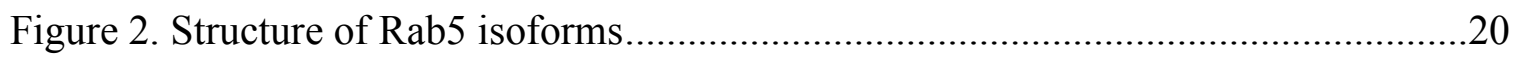

Figure 3. Family of Vps9 domain containing proteins ......................................................21

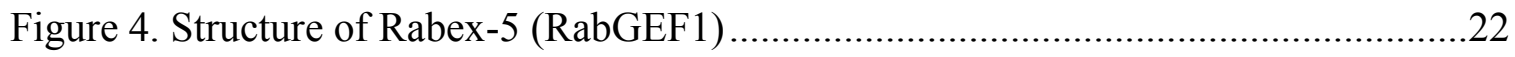

Figure 5. Effect of insulin receptor kinase inhibitor on fusion between endosomes.........62

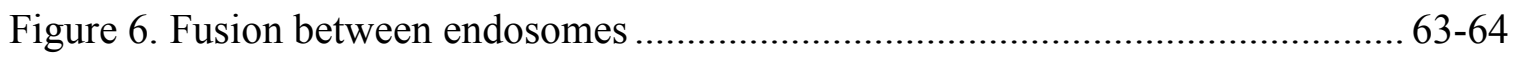

Figure 7. Morphology of in vitro fusion between endosomes ............................................65

Figure 8. Selective effect of insulin receptor kinase inhibitor on the fusion reaction .......66

Figure 9. Insulin receptor kinase inhibitor blocks endosome fusion stimulated by Rin1 ..67

Figure 10. Confocal immunofluorescence analysis of cells co-expressing Insulin receptor, Rab5: wild-type in the presence of insulin receptor tyrosine kinase inhibitor ............ 68-69

Figure 11. Confocal immunofluorescence analysis of cells co-expressing Insulin receptor, Rab5: Q79L mutant in the presence of insulin receptor tyrosine kinase inhibitor ...... 70-71

Figure 12. Insulin receptor tyrosine kinase inhibitor blocks the activation of Rab5 in

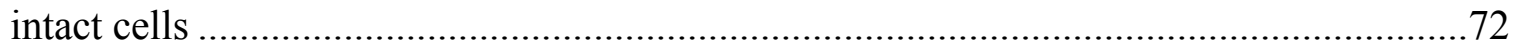

Figure 13. Effect of HNMPA-(AM) $)_{3}$ inhibitor on the tyrosine phosphorylation of Insulin receptor and recruitment of Rin1 .73

Figure 14. Confocal immunofluorescence analysis of cells co-expressing EGF-receptor and Rab5: wild-type in the presence of EGF-receptor tyrosine kinase inhibitor......... 74-75

Figure 15. Confocal immunofluorescence analysis of cells co-expressing EGF-receptor, Rab5: Q79L mutant in the presence of EGF-receptor tyrosine kinase inhibitor ......... 76-77

Figure 16. Expression and tyrosine phosphorylation of EGF-receptor in cells expressing Rab5 proteins . .78

Figure 17. Effect of AG1478 on the diameter of Rab5-positive endosomes............... 79-81

Figure 18. AG1478 inhibitor blocks the activation of Rab5 in intact cells .......................82

Figure 19. Effect of AG1478 inhibitor on the tyrosine phosphorylation of EGF-receptor and recruitment of Rin1 in intact cells. .83

Figure 20. Rin1 is not tyrosine phosphorylated by EGF .84 
Figure 21. Expression of EGF-receptor catalytically inactive mutant affects the recruitment of Rin1 to membrane upon stimulation of EGF ............................................85

Figure 22. Effect of AG1478 inhibitor on fusion between endosomes .............................86

Figure 23. AG1478 blocks endosome fusion stimulated by Rin 1 .....................................87

Figure 24. Activation of Rab5 during fluid phase and receptor-mediated endocytosis.....88

Figure 25. Activation of Rab5 during fluid phase and receptor-mediated endocytosis.....89

Figure 26. Endocytosis of EGF Receptor. ..............................................................120

Figure 27. Effect of EGF on activation of Rab5 ……………......................................121

Figure 28. Temporal activation of Rab5 based on varying amounts of EGF ……..........122

Figure 29. Effect of Temperature on Activation of Rab5 ……………….........................123

Figure 30. Effect of inhibitors of endocytosis on activation of Rab5 ..................... 124-125

Figure 31. Effect of siRNA knockdown of Caveolin and Dynamin on activation of

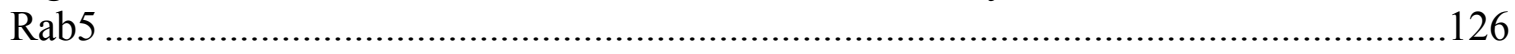

Figure 32. Effect of inhibitors of endocytosis on the formation of enlarged Rab5-posotive

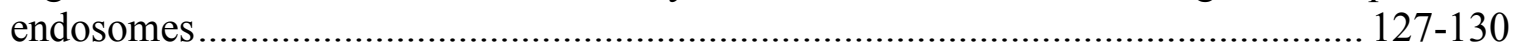

Figure 33. Effect of overexpression of Rabex-5 or Rin1 on activation of Rab5 ….........131

Figure 34. Effect of silencing of Rabex-5 or Rin1 on activation of Rab5 ......................132

Figure 35. Effect of Rabex-5 splice variants on activation of Rab5 ................................133

Figure 36. Effect of Rabex-5 splice variants on formation of Rab5 positive endosomes

Figure 37. Effect of Rabex-5 splice variants on perimeter of Rab5-positive endosomes......

Figure 38. Subcellular Distribution of Rabex-5 splice variants.....................................137

Figure 39. Summary of the adipocyte differentiation cascade ........................................158

Figure 40. Expression of Rabex-5 in 3T3-L1 cells during adipogenesis.........................159

Figure 41. Rabex-5 inhibits differentiation of mouse 3T3L1 pre-adipocytes into adipocytes . .160 
Figure 42. Rabex-5 splice variants do not affect cell proliferation. .161

Figure 43. The Vps9 domain of Rabex-5 is essential in inhibiting differentiation of 3T3L1 pre-adipocytes into mature adipocytes 162-163

Figure 44. Rabex-5 increases ubiquitination of Ras in 3T3-L1 cells .164

Figure 45. Neurite outgrowth in rat pheochromocytoma PC12 cells 165

Figure 46. Rabex-5 inhibits neurite outgrowth in rat PC12 cells $166-167$

Figure 47. Model for the role of Rabex-5 in activation of Rab5, ubiquitination of Ras and differentiation of 3T3-L1 and PC12 cells. .177 


\section{LIST OF ABBREVIATIONS AND ACRONYMS}

3T3-L1: .................. Murine "3-day transfer inoculum $3 \times 10^{5}$ cells" pre-adipocyte cell line ABL: ............................................. Abelson murine leukemia viral oncogene homolog 1

ALS: Amytrophic Lateral Sclerosis ALS2CL:

ALS2 C-terminal-like protein

AMF: Autocrine Motility Factor AMPAR: AMPK: 2-amino-3-(3-hydroxy-5-methyl-isoxazol-4yl) propanoic acid receptor
AMP activated protein Kinase ANKFY1: ANKRD27: AP: APP: APPL1: Adaptor protein containing pleckstrin homolgy domain, PTB domain and ARF: Leucine zipper Motif

ARH: Ankyrin repeat and FYVE domain-containing protein 1 Ankyrin repeat domain containing protein 27 Amyloid precursor protein AvGal:

BCA: ADP Ribosylation Factor BCR: BSA: cAMP:

CALM:

CavME: $\mathrm{CC}$ : CCP: CCV:

CDC42: CDR: C/EBP: CHOP10: CLIC:

CME:

CREB:

CTXB:

DAB2:

DBL:

DH:

DLB:

DTT:

EEA1:

EGF:

EGFR:

ERBB2:

ERK: Autosomal Recessive Hypercholesterolemia Avidin Galatosidase Bicinchoninic acid Breakpoint cluster region protein Bovine Serum Albumin cyclic AMP Clathrin assembly lymphoid myeloid leukemia protein Caveolin Mediated Endocytosis Coiled coil Clathrin Coated Pit Clathrin Coated Vesicle Cell Division Control protein 42 homolog . Circular Dorsal Ruffles CCAAT/enhancer-binding protein C/EBP homologous protein 10 .Clathrin Independent Carriers Clathrin Mediated Endocytosis cAMP response element binding protein Cholera Toxin subunit B Disabled adaptor protein 2 Defective body length .. Dbl homology Dementia associated with Lewy Bodies . Ditiotheritol .. Early Endosomal autoantigen 1 Epidermal Growth Factor Epidermal Growth Factor Receptor Erythroblastic leukemia viral oncogene homolog 2 Extracellular signal regulated kinase 
ESCRT:

Endosomal Sorting Complex Required for Transport

FL: Full Length

FYVE: Zinc finger domain named after cysteine rich proteins Fab1, YOTB, Vac1 and EEA1

GAP:

GTPase Activating Protein

GDI:

Rab GDP dissociation inhibitor

GEEC:

GEF:

GFP:

GLUT4:

GPI:

GRB2:

GSK3:

GST:

GTP:

HB:

HB-EGF:

HCC:

HER:

HGF:

HIP1:

HRP:

IGF:

IL2:

GPI-enriched Early Endosomal Compartment Guanine nucleotide Exchange Factor Green Fluorescent Protein Glucose Transporter Type 4 Glycosylphosphatidyl inositol Growth factor receptor bound protein 2 Glycogen synthase kinase 3 Glutathione S Transferase Guanine TriPhosphate Helical bundle

IR:

IRS:

LSD:

LTD:

MAPK

MBM:

MES:

MIU:

MNK:

MON1:

MVB:

NEM:

NGF:

NMDA:

NR6-E:

NR6-K:

NRDG1:

NSCLC:

NSF:

ORO:

PAR:

..Heparin-binding EGF-like growth factor Hepatocellular carcinoma associated protein Human Epidermal growth factor Receptor

Hepatocyte Growth Factor Huntingtin Interacting Protein 1 Horseradish peroxidase Insulin like growth factor .Interleukin-2 Insulin receptor Insulin receptor substrate Lysosomal Storage Disease Long Term Depression Mitogen Activated Protein Kinase Membrane binding motif 2-[morpholino] ethanesulfonic acid Motif interacting with ubiquitin Menkes disease protein Monensin sensitivity protein 1 . Multi-Vesicular Body $\mathrm{N}$-ethylmaleimide Nerve growth factor $\mathrm{N}$-Nitrosodimethylamine NR6 cells overexpressing EGFR:WT ...NR6 cells overexpressing EGFR:K712M $\mathrm{N}$-myc downstream regulated 1 Non-Small-Cell Lug Carcinoma $N$-ethylmaleimide Sensitive Factor Oil Red-O Partitioning defective proteins 
PBS:

Phosphate Buffered Saline

PC12: pheochromocytoma cells

PDGF: Platelet Derived Growth Factor

PDGFR:

PI3K:

PI3P: Platelet Derived Growth Factor Receptor
................. Phosposphatidylinositol 3-Kinase

$\mathrm{PI}(4,5) \mathrm{P} 2$ : Platelet Derived Growth Factor Receptor

PKC: Platelet Derived Growth Factor Receptor

PKD: P Protein Kinase C

PLC- $\gamma$ :

PNS: Phosphatidyl inositol $(4,5)$ bisphosphate

PPAR $\gamma:$

PR: olycystic Kidney Disease . Phospholipase $\mathrm{C}$ gamma Post nuclear supernatant

PTB: Proline rich

RA:

RABEP1: Rab GTPase-binding effector protein 1

RABEX-5:

RAC1:

RAP6:

RCC1:

RIN1:

Peroxisome proliferator-activated receptor $\gamma$

RINL:

RTK:

RXR:

Phosphotyrosine-binding domain Ras association domain

SDS-PAGE:

SH:

SHC:

SRC:

SREBP:

TGF- $\beta$ :

TRKA:

UIM:

VPS9:

VPS34:

WASP:

WAVE:

WT:

ZFYVE20:

$\mathrm{ZnF}$ : Rabaptin-5 associated Exchange factor for Rab5 Ras-related $\mathrm{C} 3$ botulinum toxin substrate 1 Rab5 Activating Protein 6 Regulator of chromosome condensation 1 Ras interference/interaction 1 .Ras interfecrence/interaction-like protein Receptor Tyrosine Kinase Retinoid $\mathrm{x}$ receptor Sodium dodecyl sulfate polyacrylamide gel electrophoresis Src homolgy domain $\mathrm{SH} 2$ adaptor protein ..Sarcoma Sterol regulatory element binding protein $1 \mathrm{c}$ Transforming Growth Factor Beta Tyrosine kinase receptor type 1 . Ubiquitin interacting motif ..Vacuolar Sorting Protein 9 ..Vacuolar Sorting Protein 34 Wiskott-Aldrich Syndrome Protein WASP family Verprolin-homologus Protein Wild Type Zinc finger, FYVE domain containing 20 .Zinc Finger 
CHAPTER 1

INTRODUCTION 


\subsection{Different routes of endocytosis}

Endocytosis is a process by which cells internalize extracellular material (in the form of nutrients, hormones, pathogens, or any other extracellular matter) that is essential for normal cell function but which is either too large (or too polar) to pass across the plasma membrane. Endocytosis of molecules has a multitude of functions in the cell, from cellular growth, division, differentiation and signal transduction, among others [McMahon \& Boucrot, 2011]. The key routes of entry into the cell are through Clathrinand Caveolin-mediated or carrier independent endocytosis (which could be either dependent or not on Dynamin), along with Macropinocytic and Phagocytic pathways (Figure 1).

Clathrin Mediated Endocytosis (CME) is the classical endocytic pathway which involves recruitment of receptor(s) into clathrin coated pits (CCPs), invagination of the plasma membrane, vesicle scission, shedding of the vesicle coat, fusion with the early endosome and sorting at the Multi-Vesicular Body (MVB) either for recycling or degradation in the lysosomes [Sorkin, 2004]. The membrane coat is made up of clathrin, various adaptor proteins and phosphatidylinositol $(4,5)$ bisphosphate or $\mathrm{PI}(4,5) \mathrm{P} 2$. The pathway is mainly regulated by Dynamin and Rab proteins, which respectively are responsible for the scission of the $\mathrm{CCP}$ and activation of their respective effectors in turn regulating vesicle budding, motility and fusion [Zerial \& McBride, 2001]. CME is used by all known eurkaryotic cells and is very versatile because many different cargoes can be packaged therein using a range of accessory adaptor proteins. Although clathrincoated vesicles can also be formed from other membranous compartments and used for various cellular processes (such as endosomal sorting complex required for transport, 
ESCRT-dependent sorting at endosomes [Raiborg et al., 2001], protein secretion from the trans-Golgi network [Deborde et al., 2008] and mitosis [Royle et al., 2005]), the term CME is used to refer only to the intake through vesicles formed from the plasma membrane [McMahon \& Boucrot, 2011].

Clathrin has been shown by numerous studies to play a key role in the endosomal sorting process [van Dam \& Stoorvogel, 2002; Raiborg et al., 2002]. Depletion of clathrin from cells leads to an ablation of CCP formation and subsequent endocytosis of the receptor. Adaptor proteins, such as adaptor protein 2 (AP2), have also been shown to play a key role in CME as depletion of AP2 has been shown to inhibit formation of the clathrin coat [Sorkin, 2004; Maldonado-Baez \& Wendland, 2006; Motley et al., 2003; Bourcot et al., 2010]. By binding to the head groups PI(4,5)P2 and PI4P (Phosphatidylinositol 4-Phosphate), AP2 acts as a link between clathrin and the underlying membrane and hence its depletion, though may not inhibit CME completely, will lead to a decrease in CME [Bourcot et al., 2010]. Alternative adaptor proteins (such as Epsin, $\beta$-arrestin, AP180/CALM (adaptor protein 180/Clathrin assembly lymphoid myeloid leukemia protein), Hip1 (Huntingtin Interacting protein 1), Dab2 (disabled adaptor protein 2), ARH (autosomal recessive hypercholesterolemia)) are also known to exist and play a key role in CME. Inhibition of AP2 only knocks down CME dependent EGFR (Epidermal growth factor receptor) uptake by about 50 percent, which makes sense because the aforementioned adaptor proteins themselves can bind to clathrin, cargo as well as phosphoinositides. In addition, during the depletion of AP2 there would be less clathrin and subsequently less CCPs on the plasma membrane, hence it is logical that CME should decrease but should not be completely inhibited [Huang et al., 2004; 
Lakadamyali et al., 2006; Sigisimund et al., 2008]. Once dynamin pinches off the CCP from the plasma membrane, auxilin and hsc70 work in concert to release the clathrin coat from the excised vesicle [Doherty \& McMahon, 2009].

Although CME is the most widely studied and best understood model of endocytosis, cargo can be endocytosed via pathways independent of clathrin. Moreover these pathways function in a cholesterol-dependent manner, in contrast to CME which is cargo driven. One of the plausible reasons for employing a variety of different forms of endocytosis is to perhaps deliver cargo to distinct intracellular destinations by creating endosomal compartments with specific membrane protein composition, which would therefore direct the variety of distinct intracellular events.

During Caveolin Mediated Endocytosis (CavME) (Figure 1), the coat is composed by oligomerization of caveolin that in turn forms lipid rafts which are budding inward and directed toward caveosomes that can be directed to early endosomes and hence converge with the aforementioned classical CME pathway in a process that is regulated by Rab5 [Parton \& Simons, 2007; Pelkmans et al., 2004]. The CavME pathway is activated through PKC and SRC (sarcoma) family kinases. Caveolae are the 80nm invaginations (analogous to CCPs from CME) through which CavME occurs with the help of dynamin as the GTPase required for the vesicle scission from the cell membrane. CavME have been observed in smooth muscle, pneumocytes, fibroblasts, adipocytes and endothelial cells [Parton \& Simons, 2007].

Cells can also internalize cargo in a clathrin- and caveolin-independent manner (Figure 1). The first is through a pathway regulated by flotillin, which is homologous to caveolin, but whose cargo has been found in plasma membrane domains distinct from 
caveolae. Epidermal Growth Factor (EGF) also stimulates the flotillin pathway, but interestingly it has not been shown to associate with EGFR. Cell surface proteoglycans are internalized in a CavME and CME independent manner with vesicle scission being controlled by dynamins [Paynet et al., 2007]. On the other hand, Glycosylphosphatidylinositol-anchored proteins are taken up by pathways independent of clathrin and caveolin, known as CLIC/GEEC (Clathrin and Caveolin Independent Carriers/GPI-anchored protein enriched Early Endocytic Compartment) pathways, and are regulated by CDC42 (Cell Division Control protein 42 homolog) and Arf1 (ADP ribosylation factor 1) through tubular plasma invaginations [Kumai \& Mayor, 2008; Doherty \& Lundmark, 2009]. The aforementioned pathways, however, do not always require dynamin to pinch off from the plasma membrane [Lamaze et al, 2001] and have been shown to internalize SV40 virions, CTxB (Cholera Toxin subunit B), other sphingolipids, IL2 (Interleukin-2) receptors, AMF (autocrine motility factor), endothelin, growth hormones as well as other molecules [Kirkham \& Patron, 2005]. Interestingly vesicles from this pathway can bypass the canonical Rab5 endosomes.

Molecules can also be macropinocytosed into the cell via macropinosomes, which are membrane protrusions that can fuse with themselves or back with the plasma membrane and create endocytic vesicles that are capable of internalizing extracellular fluid (along with other cargo) that is greater than $1 \mu \mathrm{m}$ in diameter and independent of clathrin but dependent on cholesterol (Figure 1). These are regulated by Rac1, actin, Arf6 and the WAVE (WASP family Verprolin-homologus protein) complex and initiated by growth factors, for which Rab5 is a crucial controller of [Lanzetti et al., 2005]. Numerous proteins such as PI3K, Ras and Src have all been shown to stimulate macropinocytosis 
[Veithen et al., 1996]. It has also been shown that in response to high concentration of ligand (EGF, PDGF (platelet derived growth factor) or HGF (hepatocyte growth factor)) certain receptor tyrosine kinases (EGFR, PDGFR) move along the dorsal surface of membranes on structures that have been termed circular dorsal ruffles (CDRs) whose internalization appears to be dependent on cortactin and dynamin. What is interesting is that the receptors become internalized very rapidly through tubular rather than circular structures that are independent of clathrin or caveolin [Li et al., 1997; Krueger et al., 2003; Doherty \& McMahon, 2009]. The significance of the aformenetioned for the cell is probably to desensitize cells to large concentrations of ligand.

\subsection{Abnormalities associated with derailed endocytosis}

Aberrant endocytosis can very easily lead to pathogenesis associated with neurodegenerative diseases, cancer metastasis, kidney disease, as well as insulin resistance and other metabolic disorders. For example, Cdc42-gtp targets junctional proteins to lysosomes, while cdc42-gdp targets them to recycling endosomes that contain Rab13. Furthermore, overexpression of $\mathrm{Cdc} 42$ has been shown to be associated with advancement of testicular cancer [Kamai et al., 2004]. On the other hand, EGF is able to phosphorylate PAR3 (Partitioning defective proteins) and establish tight junction polarity, while HER2 (Human Epidermal growth factor Receptor or the oncogenic receptor ERBB2 (Erythroblastic leukemia viral oncogene homolog 2)) binds to PAR6, recruits $\alpha$ PKC (Protein Kinase C) and thereby disrupts polarity [Aranda et al., 2006; Wang et al., 2006]. An E3 ubiquitin ligase, von Hippel-lindau protein on the other hand, targets $\alpha \mathrm{PKC}$ for degradation and therefore inhibits the polarity disrupting effects of 
HER2 [Okuda et al., 2001]. E-cadherin can be internalized by CME, as well as CavME through EGF stimulated macropinocytosis [Bryant et al., 2007]. If E-cadherin can not trap B-catenin at the cytoplasm, the latter translocates to the nucleus and activates the wnt pathway, which is known to control polarity and cell migration [Nelson \& Nusse, 2004].

Rab4-GTP interacts within trans-Golgi network via N-Myc Down Regulated Gene 1, also known as NDRG1 (a suppressor of prostate and breast metastasis) to recycle Ecadherin, while Rab11 (when residing in the recycling endosomes) regulates the trafficking of E-Cadherin to basolateral surface from the Golgi network along with activating Rap1 [Balzac et al., 2005; Kachhap et al., 2007]. Because endosomal acidification is required for endocytosis of low molecular weight proteins in the proximal convoluted tube, aberrant endocytosis of the machinery responsible for acidification of endosomes has been shown to the be one of the main causes of renal Fanconi syndrome and Dent's disease [Devuyst, 2010].

Regulation of endocytosis also plays a role in pathogenesis of type II diabetes since GLUT4 (Glucose Transporter Type 4, which is the principal carrier responsible for glucose transport) in the cell membrane is controlled by both CME as well as caveolin independent (but cholesterol and dynamin dependent) endocytosis [Antonescu et al., 2008], and hence mis-regulation of either pathway could lead to insulin resistance.

Atherosclerosis is a condition in which there is an accumulation of cholesterol without subsequent clearing that subsequently leads to the thickening of the arterial wall. However, if an inhibitor of dynamin (a GTPase involved in the scission of budding vesicles) is employed, reduction in atherosclerosis is achieved by enhancing TGF- $\beta$ and thereby offsetting the repressed awareness caused by hypercholesterolemia [Chen et al., 
2009] supporting the evidence that receptor mediated endocytosis is involved in pathogenesis of atherosclerosis.

It has also been shown that endocytosis is involved in pathogenesis of muscular dystrophy as caveolin specifically inhibits dysferin endocytosis thereby causing its retention at the cell surface (since mutations in the caveolin and dysferin are associated with limb girdle muscular dystrophy type 2b) [Hernandez-Deviez et al., 2008].

Lysosomal storage disease (LSD) is a group of genetic diseases characterized by the presence of enlarged late endosomes and lysosomes in neuronal cells potentially caused by either faulty late endosomal tubular trafficking, reduced lysosome-mediated retrograde transport and exocytosis or abnormal endocytic lysosomal sorting [Zhang, 2008]. Alzheimer's disease is another neurodegenerative disease in which aberrant endocytosis of Amyloid Precursor Protein (APP) and subsequent accumulation of the truncated and ultimately toxic form $A \beta 42$ leads to buildup of $\beta$-amyloid plaques and pathogenesis of the disease [Zhang, 2008].

Another neurodegenerative disorder associated with abnormal endocytosis is amytrophic lateral sclerosis (ALS). The ALS2 protein is one of the GEFs associated with activation of Rab5 (via the VPS9 domain) as well as Rac1. Recently, it has been shown that the loss of functional VPS9 domain of the ALS2 protein is enough to stimulate ALS [Otomo et al., 2003].

Menkes Disease is associated with an abnormally high absorption of copper in kidneys and the small intestine which then leads to copper deficiency in other tissues such as the brain. Although copper is an essential trace element, high concentrations of it are toxic, therefore it needs to be under strict homeostatic control, which is done through 
both clathrin dependent and indpenedent endocytosis of MNK (Menkes disease protein) copper-transporting P-type ATPase [Lane et al., 2004].

Hence, it is evident that a thorough understanding of the mechanisms underlying the various types of endocytosis are essential for elucidating the pathogenesis of numerous debilitating conditions ranging from cancer to several neurodegenerative and metabolic disorders.

\subsection{Endocytosis of Epidermal Growth Factor Receptor (EGFR)}

Epidermal Growth Factor Receptor (EGFR or ERBB1) is a member of the Receptor Tyrosine Kinase (RTK) family of receptors which also include the ERBB2/HER2, ERBB3 and ERBB4 receptors. The structure of these receptors is such that they contain an extracellular ligand-binding domain composed of two cysteine rich regions (for ligands ranging from EGF, TGF $\alpha$ (Transforming Growth Factor), amphiregulin, HB-EGF (Heparin-binding EGF-like growth factor) and a number of virally-encoded factors), an alpha helical transmembrane domain, leading to an intracellular domain. The intracellular domain is of interest because it possesses tyrosine kinase activity that is comprised of a PKC/MAPK attenuation site followed by an SH1 (Src homology 1), and a C terminal tail which adaptor proteins which contain SH2 (Src Homology 2) and PTB (Phosphotyrosinebinding) domains can interact with [Wells, 1999; Woodworth et al., 2005]. Upon binding of a ligand, EGFR homodimerization or heterodimerization (with other members of the EGFR family) occurs, leading to the activation of the tyrosine kinase domain and autophosphorylation of tyrosine residues which activate MAPK, PKC and PI3K signaling pathways via various adaptor proteins [Wells, 1999]. It is known that if EGF stimulation 
is greater than $10 \mathrm{ng} / \mathrm{ml}$, majority of the EGFR is destined for degradation via clathrin independent pathway(s), whereas if stimulation is less than $2 \mathrm{ng} / \mathrm{ml}$, majority of the receptor is destined to be recycled to the cell surface via CME (Figure 1) [Sigisimund et al., 2005; Sigisimund et al., 2008;]. However, internalized receptor through either pathway is destined for early endosome sorting [Sigisimund et al., 2008]. In the CME pathway, upon binding of EGF, receptor dimerizes over the clathrin coated pit and gets endocytosed. What follows is multiubiquitination of EGFR and subsequent degradation of both the receptor and the ligand at Multi-Vesicular Body [Levkowitz et al., 1999]. Interestingly, if TGF $\alpha$ or HB-EGF binds to the EGFR, what results is a hypoubiquitination of the EGFR (because of their instability in acidic compartments), leading to recycling of the receptor to the cell surface and continuous signaling, which is a hallmark observed in lung, ovarian, colon and prostate cancer malignancies [Ebner \& Derynck, 1991; Nicholson et al., 2001].

\subsection{Disorders arising from misregulation of EGFR}

EGFR is also involved in numerous signaling cascades in which it is known to activate the Mitogen-Activated Protein Kinase (MAPK), PLC $\gamma$, PLD, PI3K-Akt pathways, as well as activation of transcription signaling pathways [Kumar et al., 2008]. Therefore, aberrant activation or trafficking of the receptor can lead to various oncogenic and malignant diseases, especially Non-Small-Cell Lung Cancer (NSCLC) which is the leading cause of cancer death in North America [Jemal et al., 2005]. However, inhibition

of the EGFR using either siRNA or synthetic inhibitors (such as PD153035) and examination of affected genes (using a microarray) have shown that inactivating EGFR 
also has an effect on gene expression of various cellular processes ranging from proinfalmatory responses, genes that promote cell attachment, apoptosis as well as those which inhibit cell cycle progression [Woodworth et al., 2005]. EGFR has also been implicated to be involved in PKD (Polycystic Kidney Disease) where there is an overexpression and mislocalization of EGFR that can be reversed with use of pharmacological or genetic inhibitors of EGFR tyrosine kinase activity [Torres et al., 2003]. Lastly, it has been shown that constitutive activation of the EGFR can lead to cirrhotic liver disease and can ultimately lead to Hepato Cellular Carcinoma (HCC) [Komuves et al., 2000; Buckley et al., 2008].

\subsection{Role of small GTPases in intracellular trafficking}

The Rab family of proteins is a subset of a superfamily of Ras proteins, which are low molecular weight GTPases $(20-30 \mathrm{kD})$ that have the ability to switch between GTP (active-membrane bound) and GDP (inactive-cytosolic) bound form, and in that sense act as molecular switches [Li \& Stahl, 1993; Barbieri et al., 1994; Li et al., 1994; Barbieri et al., 1996]. Including various isoforms, currently the family comprises of over 60 members [Stenmark \& Olkonen, 2001; Baskys et al., 2007]. All Rab proteins have a GTPase domain and are isoprenylated at their $\mathrm{C}$ terminus for integrating into different membranes. Through distinct localization on various vesicles, and organelles and by cycling through GTP/GDP bound forms, Rabs are able to recruit and activate various

effector proteins, both spatially and temporally, and hence behave as key regulators of trafficking processes in both endocytic and biosynhtetic pathways (vesicle budding, cargo sorting, tethering, as well as docking and fusion of vesicles with acceptor membranes). 
The cycling between the active (GTP-bound) and inactive (GDP-bound) forms depends on auxiliary elements that control association with membrane(s), binding of nucleotide(s) and GTP hydrolysis [Delprato et al., 2004].

\subsubsection{Rab5}

The Rab5 protein is ubiquitously expressed and has been shown to regulate transport from plasma membrane to the early endosome. It has also been shown that the protein is required for homotypic early endosome fusion and its overexpression has been shown to increase the rate of endocytosis [Li \& Stahl, 1993; Barbieri et al., 1994;, Li et al., 1994; Barbieri et al., 1996; Chiariello et al., 1999]. It has also been shown to be involved in regulating generation of phophotidylinositol-3-phosphate lipid which is enriched in the early endosome membrane [Murray et al., 2002] and the motility of early endosomes on actin and microtubule filaments [Pal et al., 2006], as well as activating signaling pathways from the early endosome [Schenck et al., 2008]. There are three isoforms of Rab5, those being Rab5a, Rab5b and Rab5c (Figure 2), which show about $80 \%$ sequence homology and all of which have been shown to be involved in regulating dynamics of early endosome fusion [Bucci et al., 2010]. Hence, the presence of Rab5 isoforms may reflect an evolutionary need to ensure Rab5 function even if aberrant mutations arise. However, it has been shown that they are differentially regulated in response to different stimuli and that phosphorylation could be responsible for a fine regulation of endocytosis in vivo due to the fact that each possess a common Ser/Thr kinase site (at position 123), but are differentially phosphorylated by specific kinases (ERK1 preferentially phosphorylating Rab5a, while p34cdc42 kinase preferentially 
phosphorylating Rab5b) [Chiarielo et al., 1999]. As with other small GTPases, Rab5 contains a highly conserved phosphate-binding loop (P-loop) made up of ${ }^{27}$ GESAVGKS $^{34}$ residues which have been shown to be important in GTP hydrolysis, whereas $\mathrm{N}$ and $\mathrm{C}$ termini are hypervariable. The $\mathrm{C}$ terminus is usually isoprenylated for targeting to particular membranes, as is the case with Rab5. Rab5 is very tolerable in its mutations at the $\mathrm{N}$-terminus, meaning that the $\mathrm{N}$ terminal sequence is not an essential component required for Rab5 function, whereas mutations in the $\mathrm{C}$ terminus (especially the two cysteins of the CCSN tetrapeptide motif) eliminate the post-translational isoprenylation, membrane assosication and biological function of Rab5 [Li \& Stahl, 1993; Zhu et al., 2003].

\subsubsection{Guanine Nucleotide Exchange Factors}

Guanine Nucleotide Exchange Factors (GEFs) are proteins that activate small GTPases by catalyzing the exchanging of GDP by a GTP. All Rab5-GEFs contain a highly conserved Vps9 domain (Figure 3) through which they interact and activate Rab5. Some of the currently known Rab5-GEFs are Alsin, ALS2CL (ALS2 C-terminal-like protein), AnkRD27 (Ankyrin repeat domain containing protein 27), Rabex-5 (Rabaptin-5 associated Exchange factor for Rab5), Rap6 (Rab5 activating protein 6), Rin1 (Ras interference/interaction 1), Rin2, Rin3 and RinL (Ras interference/interaction-like protein) (Figure 3) [Saito et al., 2002; Kajiho et al., 2003; Otomo et al., 2003; Hunker et al., 2006; Galvis et al., 2009; Jovic et al., 2010]. GTPase Activating Proteins (GAPs) speed up the intrinsic GTPase activity of Rab proteins, in turn inactivating them, and in 
doing so allow Rab proteins to complex with their respective GDP-dissociation inhibitors (GDIs) in order to complete the cycle [Delprato et al., 2004].

\subsubsection{Ras Interference/Interaction protein}

As other Rab5 GEFs, the Ras Interference/Interaction (Rin) group of proteins all contain a Vps9 domain (Figure 3), but also a Ras binding domain that facilitates allosteric regulation of Rab5 exchange activity by GTP bound Ras [Tall et al., 2001; Delprato et al., 2004]. The Rin 1 protein also interacts with EGFR (and other receptor tyrosine kinases) via the SH2 domain, as well as Rab5 through the VPS9 domain, and since it acts as a Rab5GEF, it is plausible to assume that Rin1 plays a key role in recruitment of Rab5 along with its subsequent activation through ligand dependent stimulation of EGFR (as well as other RTKs). Furthermore, Rab5 and Rin1 localize to both plasma membrane and endosomes [Barbieri et al., 2003]. Pathologically, Rin1 has also been shown to enhance the oncogenic activity of BCR-ABL (Breakpoint cluster region protein-Abelson murine leukemia viral oncogene homolog 1) by interacting with the BCR-ABL fusion protein and hence speed up the BCR-ABL-induced leukemia in mice [Han et al., 1997; Delprato et al., 2004].

\subsubsection{Rabaptin-5 associated Exchange factor for Rab5}

Another well studied GEF for Rab5 is the Rabaptin-5 associated Exchange factor for Rab5 (Rabex-5), more commonly known as Rabex-5 (sometimes referred to as RabGEF1). David Lambright's group has crystalized the Rabex-5 structure and identified it to be composed of an N-terminal A20 type zinc finger domain and a motif interacting with ubiquitin (MIU domain), a membrane binding domain, a helical bundle and a Vps9 
domain (which together comprise the GEF catalytic core), and a $\mathrm{C}$ terminal coiled coil and proline rich domains (which form a complex with Rabaptin-5 and indirectly target Rabex-5 to early endosomes) (Figure 4) [Delprato et al., 2004; Aikawa, 2012; Shin et al., 2012]. The same group also found that the amino acids D313, P317 and Y354 are all highly conserved among the Vps9 containing proteins, and that these residues are vital in maintaining full GEF activity of Rabex-5 [Delprato et al., 2004]. The discriminate binding of Rabex-5 to Rab5 lies in the requirement for a small non-acidic residue preceding F56 residue in the switch I-GxxF motif of Rab5. Substitution of this alanine with aspartic or glutamic acid (which are conserved in nearly all Rab GTPases) results in a 200 -fold decline in catalytic efficiency. Interestingly, in comparison to other GEFs, full length Rabex-5 has a very weak in vitro exchange activity, but increases tremendously when complexed to Rabaptin-5 or when only the helical bundle-Vps9 domain catalytic core is employed. One of the explanations for this could be the fact that regulatory elements which are found C-terminus (residues 415-425) can act as autoinhibitors of Rabex-5 GEF activity by overlapping the binding site for Rabaptin-5 [Delprato \& Lambright, 2007]. It has been demonstrated that Rabex-5 activates Rab5 (through its catalytic core) and in doing so recruits Rabaptin-5, which then binds to the GTP bound form of Rab5 and recruits Rabex-5 in a positive feedback loop. This in turn stabilizes activated Rab5 on early endosomes and can therefore recruit other effector proteins of Rab5 (RabEP1-2 (Rab GTPase-binding effector proteins 1-2), ANKFY1 (Ankyrin repeat and FYVE domain-containing protein 1), MON1A-B (Monensin sensitivity protein 1), ZFYVE20 (zinc finger, FYVE domain containing 20), VPS34 (Vacuolar Sorting Protein 34), EEA1 (Early Endosomal autoantigen 1), Rabenosyn-5, APPL1 (Adaptor protein 
containing pleckstrin homolgy domain, PTB domain and leucine zipper Motif), APPL2) to the early endosome where specialized trafficking functions can be carried out [Jovic et al., 2010]. The Rabex-5 protein can also down regulate EGFR activity since it translocates to the plasma membrane after stimulation with EGF and colocalizes with EGFR at various stages of endocytosis through two distinct ubiquitin binding sites (A20 zinc finger with E3 ubiquitin ligase activity and an inverted UIM (Ubiquitin interacting motif) with ubiquitin binding activity) [Lee et al., 2006; Penengo et al., 2007; Delprato \& Lambright, 2007; Xu et al., 2010]. Rabex-5 has also been shown to inhibit Ras signaling via the ubiquitin ligase activity of $\mathrm{ZnF}$ domain and not the GEF domain [Colicelli, 2010; Yan et al., 2010]. Overexpression of Rabex-5 leads to an increased localization of Ras with EEA1 on endosomes, which has previously been shown to be a sign of Ras ubiquitination, while silencing Rabex-5 leads to inhibition of the aforementioned colocalization [Jura et al., 2006; Colicelli, 2010; Xu et al., 2010]. By restricting Ras signaling, Rabex-5 has been shown to be important in establishing organism size, wing vein pattern and eye versus antennal fate in Drosophila [Yan et al., 2010]. The same group has also shown that loss of Rabex-5 leads to death of flies as giant larvae or giant pupae often containing melanotic tumors [Yan et al., 2010]. Furthermore, Rabex $-5^{-1-}$ mice show a substantial increase in prenatal mortality, skin inflammation, increased numbers of skin mast cells and elevated serum levels of IgE and histamine [Tam et al., 2004; Delprato \& Lambright, 2007]. 


\subsection{Disorders associated with aberrant activation of Rab5}

As Rab5 is a key regulator of the early endocytic process, it has been implicated in numerous diseases. First, Rab5 has been associated with DLB (Dementia with Lewy Bodies), since neuronal cell death has been associated with increased $\alpha$-synuclein binding to Rab5a and its endocytosis, while expression of GTPase deficient Rab5a results in incomplete endocytosis of $\alpha$-synuclein and therefore a decrease in cytotoxicity [Sung et al., 2001]. Second, Rab5b has been shown to reduce NMDA toxicity by up-regulating endocytosis of AMPA and NMDA receptors, which would thereby render these receptors ineffective and insensitive to excitotoxic stimulation [Baskys et al., 2007]. In Alzheimer pathology, Rab5 increases $\beta$-cleaved amyoloid precursor levels and $A \beta$ production [Seabra et al., 2002]. When it comes to infections, it has been shown that in Listeria monocytogenes macrophage infections, Rab5 suppression enhances survival of Listeria, while overexpression has been shown to increase intracellular degradation of the pathogen [Hashim et al., 2000]. In Salmonella infections, it has been shown that phagosomes containing the internalized live bacteria acquire transferrin receptors and retain Rab5 and Rab18, and in doing so escape the default pathway to the lysosomes by associating with the late endosomes and maturing in specialized compartments [Hashim et a., 2000]. Lastly, Rab5 has also been implicated to be involved in cancer metastasis, amyotrophic lateral sclerosis 2 (ALS2), Long Term Depression (LTD), insulin resistance syndrome and Huntington's disease [Bao et al., 1998; Yu et al., 1999; Hadano et al., 2001; Devon et al., 2001; Pal et al., 2006; Baskys et al., 2007].

Since Rab5 has been shown to be implicated in numerous devastating diseases, further research on both the mechanism of activation/inactivation as well as function of 
this protein and its effectors should provide great insight into elucidating the pathogenesis of numerous diseases. Therefore, the aims of this dissertation will be to obtain a better understanding of Rab5 activation via tyrosine kinase receptor internalization, as well as thre role of its key activator Rabex-5 in two key biological processes, namely adipogenesis and neurogenesis. The completion of these aims will demonstrate that the tyrosine kinase activity of receptor tyrosine kinases is required for activation of Rab5; that Rab5 can be activated via at least 3 different endocytic pathways of EGFR internalization; and lastly, that Rabex-5, inhibits both adipogenesis (in 3T3-L1 cells) and neurogenesis (in PC12 and SK-N-MC cells) by promoting degradation of internalized signaling receptor (which under adipo- or neurogenic conditions is normally found inside a signalling vesicle), as well as through of Ras. Finally, this dissertation begins to explore the evolutionary redundancy of Rab5 activation, because three naturally occurring splice variants of Rabex-5 are accessible to activate Rab5 in a potential fail safe mechanism.

Parts of this dissertation have been published in peer reviewed journals. Chapter one contains work previously published in Jozic \& Barbieri, 2010. On the other hand, Chapter two was published as two separate articles, one pertaining to to Insulin-Receptor (Jozic et al., 2011) and the other to EGF-Receptor (Jozic et al., 2012). 

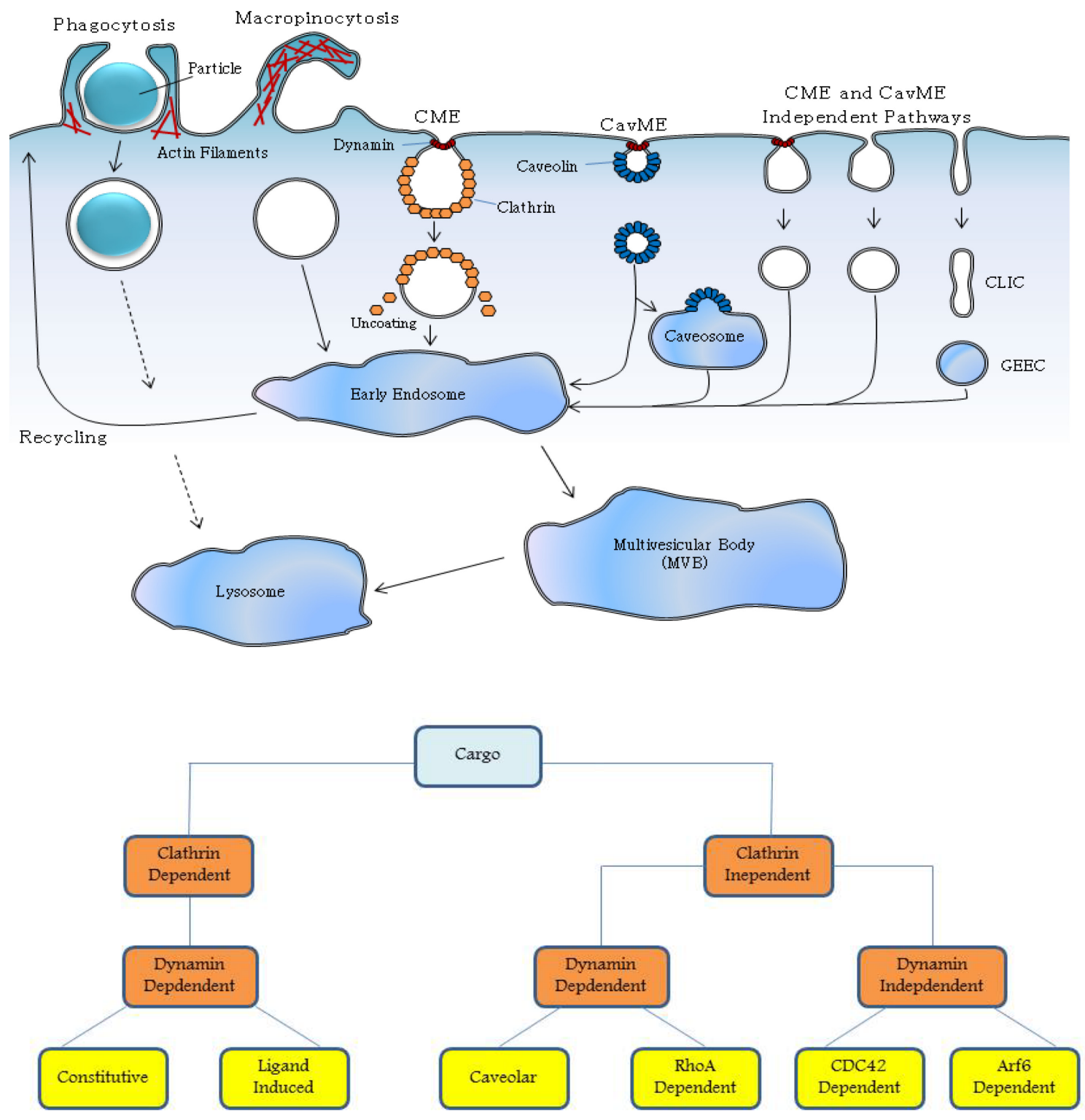

Figure 1. Pathways of EGF-Receptor Endocytosis. EGFR can be endocytosed through clathrin dependent or clathrin independent pathways (with the latter being composed of CavME and CLIC). Clathrin dependent endocytosis occurs predominantly when there is a low concentration of EGF at the cell surface and primarily results in recycling of EGFR via Rab5 dependent early endosome and recycling endosome. Clathrin indendent pathways occur predominantly when there is a high concentration of EGF at the cell surface and primarily result in degradation of EGFR. If the pathway is dependent on caveolin the internalized vesicle will fuse with the early endosome in Rab5 dependent manner prior to fusing with the multivesicular body and then degraded in the lysosome. However, if the pathway is independent of caveolin, EGFR containing vesicle may bypass the Rab5-early endosome but is still destined for degradation in the lysosome. 


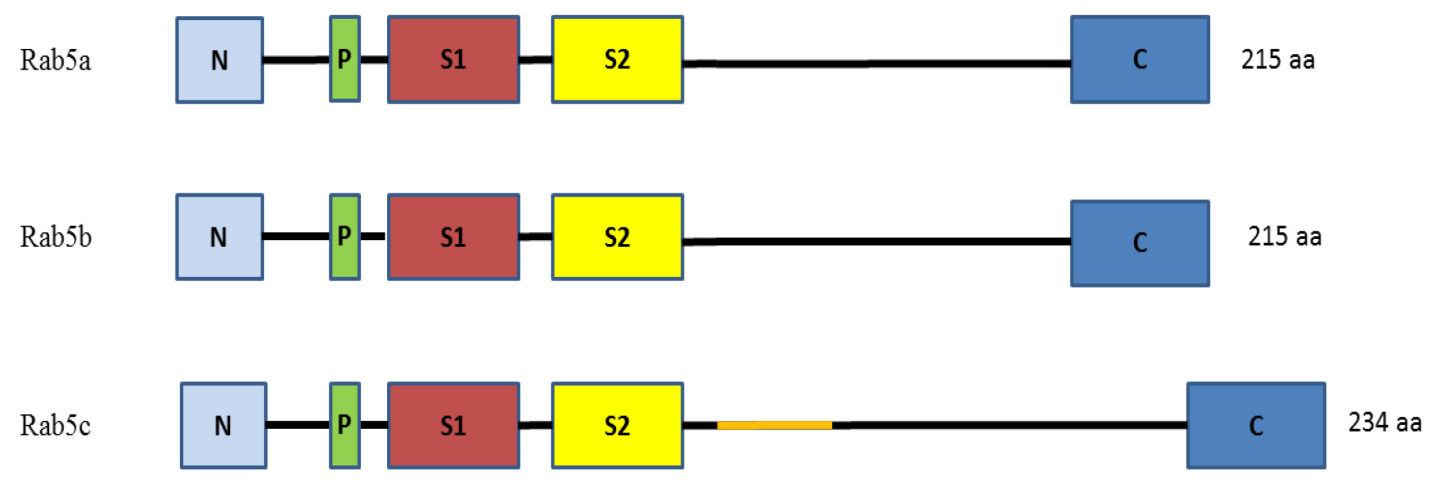

Figure 2. Structure of Rab5 isoforms. All isoforms have highly conserved P-loop (residues 27-34), Switch 1 (residues 44-64) and Switch 2 (residues 76-94) regions but differ in their N- (residues 1-16) and C-terminal (residues 184-215) hypervariable domains. With the exception of an extra 19 aa fragment in Rab5c (149-164), the three isoforms exhibit an approximate $80 \%$ sequence homology, and are all isoprenylated at their $\mathrm{C}$ terminus. 


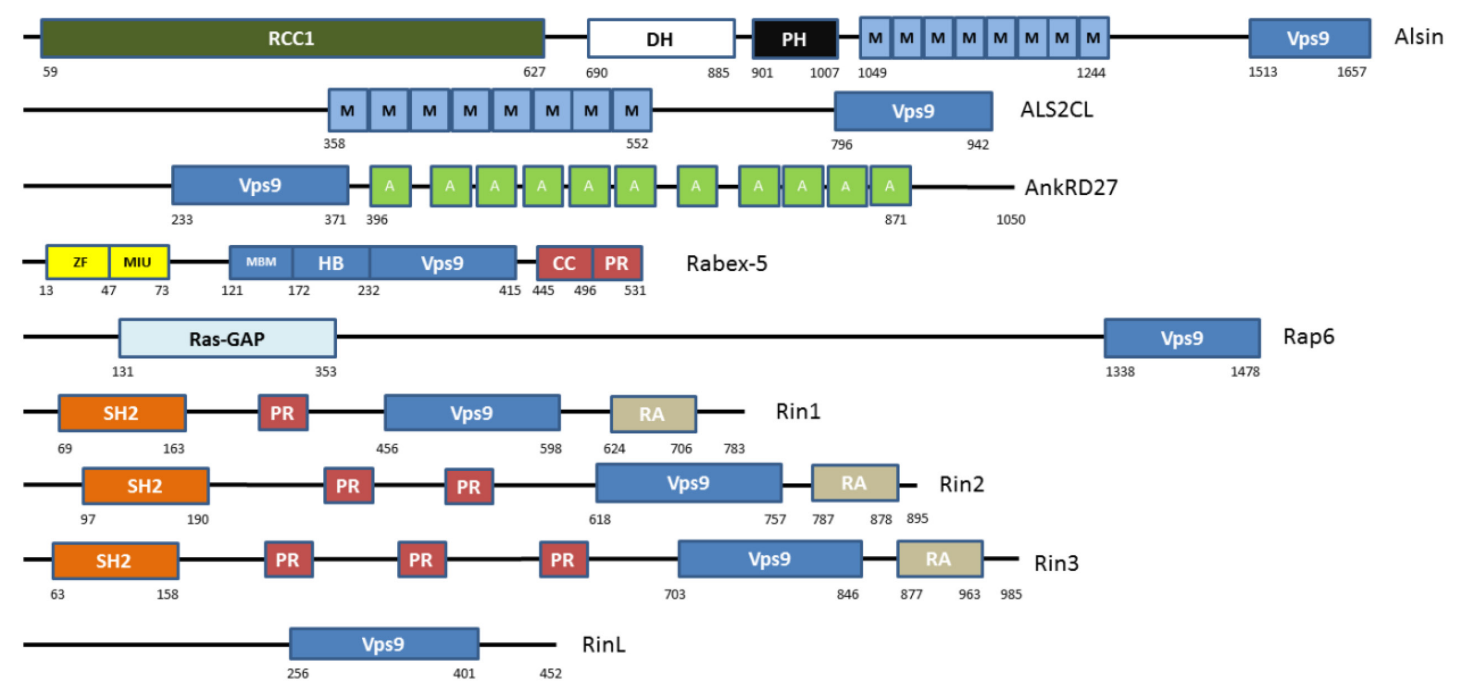

Figure 3. Family of Vps9 domain containing proteins. ALS2 C-terminal-like protein (ALS2CL); AnkRD27 (Ankyrin repeat domain containing protein 27); Rabaptin-5 associated Exchange factor for Rab5 (Rabex-5); Rab-activating protein 6 (Rap6); Rin1/2/3 (Ras interaction/interference protein) 1/2/3; Ras interaction/interference-like protein (RinL); Zinc Finger (ZF); Motif interacting with Ubiquitin (MIU); Membrane binding domain (MBM); Helical Bundle (HB); Vacuolar Sorting Protein 9 (Vps9); Coiled Coil (CC); Proline Rich (PR); Ankyrin1 (A); GTPase Activating protein for Ras (RasGAP); Regulator of Chromosome Condensation 1 (RCC1); Dbl Homology (DH); Pleckstrin Homology (PH); Membrane Occupation and Recognition Nexus repeat containing 1 (M); Src Homology 2 (SH2); Ras Association (RA). 


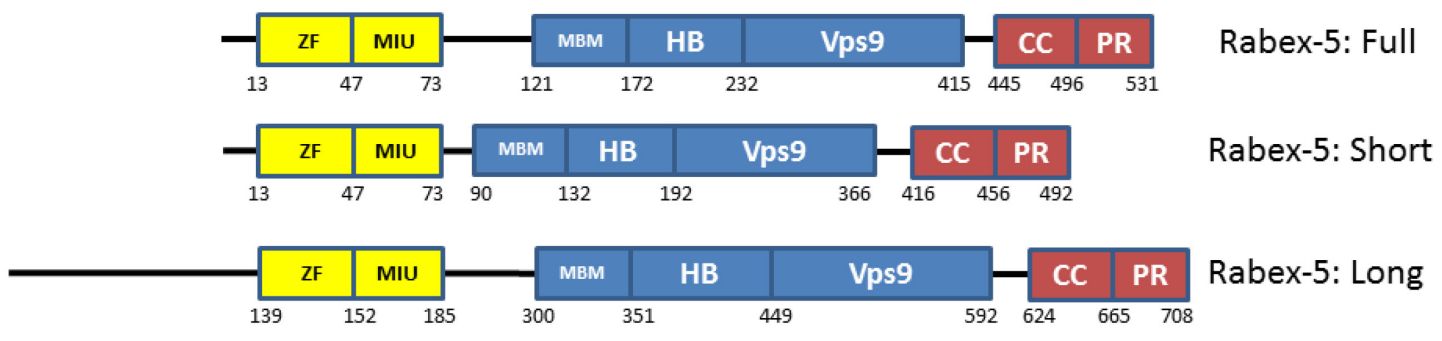

Figure 4. Structure of Rabex-5 (RabGEF1). N-terminal A20 type zinc finger domain (ZF) and a motif interacting with ubiquitin (MIU domain) in tandem serve as an E3 ubiquitin ligase; membrane binding domain (MBM); a helical bundle (HB) and a Vps9 domain which together comprise the GEF catalytic core; $C$ terminal coiled coil (CC) and proline rich (PR) domains which form a complex with Rabaptin-5 and indirectly target Rabex-5 to early endosomes. 


\subsection{References}

Aikawa Yoshikatsu. 2012. Rabex-5 regulates the endocytic trafficking pathway of ubiquitinated neural cell adhesion molecule. J. Biol. Chem. In Press.

Antonescu C.N., Diaz M., Femia G., Planas J.V., Klip A. 2008. Clathrin dependent and independent endocytosis of glucose transporter 4 (Glut4) in myoblasts: regulation by mitochondria uncoupling. Traffic. 9: 1173-1190.

Aranda V., Haire T., Nolan M.E., Calarco J.P., Rosenberg A.Z., Fawcett J.P., Pawson T., Muthuswamy S.K. 2006. Par6-aPKC uncouples ErbB2 induced disruption of polarized epithelial organization from proliferation control. Nat. Cell Biol. 8: 1235-1245.

Balzac F., Avolio M., Degani S., Kaverina I., Torti M., Silengo L., Small J.V., Retta S.F. 2005. E-cadherin endocytosis regulates the activity of Rap1: a traffic light GTPase at the crossroads between cadherin and integrin function. J. Cell Sci. 118: 4765-4783.

Bao S., Zhu J. \& Garvery W.T. 1998. Cloning of Rab5 GTPases espressed in human skeletal muscle: studies in insulin-resistant subjects. Horm. Metab. Res. 30: 656-662.

Barbieri M.A., Li G., Colombo M.I., Stahl P.D. 1994. Rab5, an early acting endosomal GTPase, supports in vitro endosome fusion without GTP hydrolysis. J. Biol. Chem. 269(29): 18720-18722.

Barbieri M.A., Roberts R.L., Mukhopadhyay A., Stahl P.D. 1996. Rab5 regulates the dynamics of early endosome fusion. Biocell. 20: 331-338.

Barbieri M.A., Kong C., Chen P.I., Horazdovsky B.F., Stahl P.D. 2003. The Src Homology 2 domain of Rin1 Mediates its binding to the epidermal growth factor receptor and regulates receptor endocytosis. J. Biol. Chem. 278(34): 32027-32036.

Baskys A., Bayazitov I., Zhu E., Fang L., Wang R. 2007. Rab-Mediated Endocytosis linking neurodegeneration, neuroprotection and synaptic plasticity? Annals of the New York Acad. of Sci. 1122: 313-329.

Bourcot E., Saffarian S., Zhang R., Kirchhaussen T. 2010. Roles of AP-2 in Clathrin Mediated Endocytosis. PLoS ONE. 5(5) e10597. 
Bryant D.M., Kerr M.C., Hammond L.A., Joseph S.R., Mostov K.E., Teasdale R.D., Stow J.L. 2007. EGF induces macropinocytosis and SNX1-modulated recycling of ECadherin. J. Cell Sci. 120: 1818-1828.

Bucci C., Lutcke A., Steele-Mortimer O., Olkkonen V.M., Dupree P., Chiariello M., Bruni C.B., Simons K., Zerial M. 1995. Co-operative regulation of endocytosis by three Rab5 isoforms. FEBS Letters. 366: 65-71.

Buckley A.F., Burgart L.J., Sahai V., Kakar S. 2008. Epidermal Growth Factor Receptor expression and gene copy number in conventional Hepatocellular carcinoma. Am. J. Clin. Pathol. 129: 245-251.

Chen C.L., Hou W.H., Liu I.H., Hsiao G., Huang S.S., Huang J.S. 2009. Inhibitors of clathrin dependent endocytosis enhance TGFB signaling and responses. J. Cell Sci. 122(11): 1863-1871.

Chiariello M., Bruni C.B., Bucci C. 1999. The small GTPases Rab5a, Rab5b and Rab5c are differentially phosphorylated in vitro. FEBS Letters. 453: 20-24.

Coliceli John. 2010. Signal Transduction: RABGEF1 Fingers RAS Ubiquitination. Curr. Biol. 20(15): R630-R632.

Deborde, S., Perret E., Gravotta D., Deora A., Salvarezza S., Schreiner R., RodriguezBoulan E. 2008. Clathrin is a key regulator of basolateral polarity. Nature 452, 719-723.

Delprato A. \& Lambright D.G. 2007. Structural basis for Rab GTPase activation by VPS9 domain exchange factors. Nat. Struct. Mol. Biol. 14(5): 406-412.

Delprato A., Merithew E., Lambright D.G. 2004. Structure, exchange determinants, and family wide rab specificity of the tandem helical bundle and Vps9 domains of Rabex-5. Cell. 118: 607-617.

Devon R.S., Orban P.C., Gerrow K. 2001. Als2-deficient mice exhibit disturbances in endosome trafficking associated with motor behavioral abnormalities. Proc. Natl. Acad. Sci. USA. 103: 9595-9600.

Devuyst Olivier. 2010. Dent's Disease: chloride-proton exchange controls proximal tube endoxytosis. Nephrol. Dial. Transplant. 25: 3832-3835 
Doherty G.J., and R Lundmark. 2009. GRAF-1 dependent Endocytosis. Biochem. Soc. Trans. 37: 1061-106

Doherty G.J. and McMahon H.T. 2009. Mechanisms of Endocytosis. Ann. Rev Biochem. 78: $857-902$

Ebner R. \& Derynck R. 1991. Epidermal growth factor and transforming growth factor-a: differential intracellular routing and processing of ligand-receptor complexes. Cell Reg. 2: 599-612.

Galvis A., Balmaceda V., Giambini H., Conde A., Villasana Z., Fornes M.W., Barbieri M.A. 2009. Inhibition of Early Endosome Fusion by Rab5-binding defective Ras interference mutants. Arch. Biochem. Biophys. 482(1-2): 83-95.

Hadano S., Hand C.K., Osuga H. 2001. A gene encoding a putative GTPase regulator is mutated in familial amyotrophic lateral sclerosis. Nat. Genet. 29: 166-173.

Han L., Wong D., Dhaka A., Afar D., White M., Xie W., Herschman H., Witte O., Colicelli J. 1997. Protein binding and signaling properties of RIN1 suggest a unique effector function. Proc. Natl. Acad. Sci. USA. 94: 4954-4959.

Hashim S., Mukherjee K., Raje M., Basu S.K., Mukhopadhyay A. 2000. Live Salmonella modulate expression of Rab proteins to persist in a specialized compartment and escape transport to lysosomes. J. Biol. Chem. 275(21): 16281-16288.

Hatanpaa K., Burma S., Habib A. 2010. Epidermal Growth Factor Receptor in glioma: signal transduction, neuropathology, imaging and radioresistance. Neoplasia. 12(9): 675684.

Hernandez-Deviez D.J., Howes M.T., Laval S.H., Bushby K., Hancock J.F., Parton R.G. 2008. J. Biol. Chem. 283(10): 6476-6488.

Huang F., Khvorova A., Marshall W., Sorkin A. 2004. Analysis of clathrin mediated endocytosis of epidermal growth factor receptor by RNA interference. J. Biol. Chem. 279: 16657-16661.

Hunker C.M., Galvis A., Kruk I., Giambini H., Veisaga M.L., Barbieri M.A. 2006. Rab5activating protein 6, a novel endosomal protein with a role in endocytosis. Biochem. Biophys. Res. Commun. 340(3): 967-975. 
Jemal A., Murray T., Wart E., Samuels A., Tiwari R.C., Ghafoor A., Feuer E.J., Thun M.J. 2005. Cancer statistics, 2005. C.A. Cancer. J. Clin. 55: 10-30.

Jovic M., Sharma M., Rahajeng M., Caplan S. 2010. The early endosome: a busy sorting station for proteins at the crossroads. Histol. Histopathol. 25(1): 99-112.

Jozic, I. and M.A. Barbieri. 2010. Rab5 and Epidermal Growth Factor Receptor During Endocytosis by Clathrin Dependent and Clathrin Independent Pathways. Current Topics in Biochemical Research. 12(2): 59-67.

Jozic, I., Blanco G., Barbieri, M.A. 2011. Inhibition of Rab5 Activation During Insulin Receptor-Mediated Endocytosis. Curr. Cellular Biochem. 1(1): 20-32.

Jozic, I., Saliba S.C., Barbieri, M.A. 2012. Effect of EGF-receptor tyrosine kinase inhibitor on Rab5 function during endocytosis. Arch. Biochem. Biophys. 525(1): 16-24.

Jura, N., Scotto-Lavino, E., Sobczyk, A., Bar-Sagi, D. 2006. Differential modification of Ras proteins by ubiquitination. Mol. Cell. 21: 679-687.

Kachhap S.K. Faith D., Qian D.Z., Shabbeer S., Galloway N.L., Pili R., Denmeade S.R., DeMarzo A.M., Carducci M.A. 2007. The N-Myc down regulated gene 1 is a Rab4a effector involved in vesicular recycling of E-cadherin. PLOS ONE. 2: e844.

Kajiho H., Saito K., Tsujita K., Kontani K., Araki Y., Kurosu H., Katada T. 2003. Rin3: a novel Rab5 GEF interacting with amphiphysin II involved in the early endocytic pathway. J. Cell Sci. 116(20): 4159-4168.

Kamai T., Yamanishi T., Shirataki H., Takagi K., Asami H., Ito Y., Yoshida K. 2004. Overexpression of RhoA, Rac1 and Cdc42 GTPases is associated with progression in testicular cancer. Clin. Cancer Res. 10: 4799-4805.

Kirkham M. \& Patron R.G. 2005. Clathrin-independent endocytosis: new insights into caveolae and no caveolar lipid raft carriers. Biochim. Biophys. Acta. 1746: 349-363

Komuves L.G., Ferren A., Jones A.L., Fodor E. 2000. Expression of Epidermal growth factor and its receptor in cirrhotic liver disease. J.H.C. 48(6): 821-830.

Krueger E.W., Orth J.D., Cao H., McNiven M.A. 2003. A dynamin-cortactin-Arp2/3 complex mediates actin reorganization in growth factor stimulated cells. Mol. Biol. Cell. 14: 1085-1096. 
Kumai S. \& S. Mayor. 2008. ARF1 is directly involved in dynamin-independent endocytosis. Nat. Cell Biol. 10: 30-41.

Kumar A., Petri E.T., Halmos B., Boggon T.J. 2008. Structure and Clinical Relevance of the epidermal growth factor receptor in human cancer. J. of Clin. Onc. 26(10): 1742-1751

Lakadamyali M., Rust M.J., Zhuang X. 2006. Ligands for clathrin mediated endocytosis are differentially sorted into distinct populations or early endosomes. Cell. 124: 9971009.

Lamaze C., Dujeancourt A., Baba T., Lo C.G., Benmerah A., Dautry-Varsat A. 2001. Interleukin 2 receptors and detergent resistant membrane domains define a clathrin independent endocytic pathway. Mol. Cell. 7: 661-671.

Lane C., Petris M.J., Benmerah A., Greenough M., Camakaris J. 2004. Studies on endocytic mechanisms of the Menkes copper translocating P-type ATPase. Biometals. 17: 87-98.

Lanzetti L., Palamidessi A., Areces A., Scita L., and P.P. Di Fiore. 2004. Rab5 is a signaling GTPas involved in acting remodeling by receptor tyrosine kinases. Nature. 429: 309-314.

Lee S., Tsaie Y.C., Mattera R., Smith W.J., Kostelansky M.S., Weissman A.M., Bonifacino J.S., Hurley J.H. 2006. Structural basis for ubiquitin recognition and autoubiquitination by Rabex-5. Nat Struct. Mol. Biol. 13: 264-271.

Levkowitz G., Waterman H., Ettenberg S.A., Katz M., Tsygankov A.Y., Alroy I., Lavi S., Iwai K., Reiss Y., Ciechanover A., Lipkowitz S., Yarden Y. 1999. Ubiquitin ligase activity and tyrosine phoshporylation underlie suppression of growth factor signaling by c-cbl/sli-1. Mol. Cell. 4(6): 1029-1040.

Li G. \& Stahl P.D. 1993. Structure-function relationship of the small GTPase Rab5. J. Biol. Chem. 268(32): 24475-24480.

Li G., Barbieri M.A., Colombo M.I., Stahl P.D. 1994. Structural featurs of the GTPbinding defective Rab5 mutants required for their inhibitory activity on endocytosis. $J$. Biol. Chem. 269(20): 14631-14635. 
Li G., D'Souza-Schorey C., Barbieri M.A., Cooper J.A., Stahl P.D. 1997. Uncoupling of membrane ruffling and pinocytosis during Ras signal transduction. J. Biol. Chem. 272: 10337-10340.

Lynch T.J., Bell D.W., Sordella R., Gurubhagavatula S., Okimoto R.A., Brannigan B.W., Harris P.L., Haserlat S.M., Supko J.G., Haluska F.G., Louis D.N., Christiani D.C., Settleman J., Haber D.A. 2004. Activating mutations in the epidermal growth factor receptor underlying responsiveness of non-small-cell lung cancer to gefitinib. N. Engl. J. Med. 350(21): 2129-39.

Maldonado-Baez L. \& Wendland B. 2006. Endocytic adaptors: recruiters, coordinators and regulators. Tre. Cell Biol. 16: 505-513.

Mayor S. \& Pagano R.E. 2007. Pathways of clathrin-independent endocytosis. Nat. Rev. Mol. Cell Biol. 18: 459-470.

Mosesson Y., Mills G.B. and Y. Yarden. 2008. Derailed endocytosis: an emerging feature of cancer. Nat. Rev. Cancer. 8: 835-850.

Motley A., Bright N.A., Seaman M.N., and Robinsons M.S. 2003. Clathrin-mediated endocytosis in AP2 depleted cells. J. Cell Biol. 162: 909-918.

Nicholson R.I., Gee J.M., Harper M.E. 2001. EGFR and cancer prognosis. Eur. J. Cancer. 37: S9-S15.

Murray J.T., Panaretou C., Stenmark H., Miaczynska M., Backer J.M. 2002. Role of Rab5 in the recruitment of hVps34/p150 to the early endosome. Traffic. 3: 416-427.

Nelson W.J. \& Nusse R. 2004. Convergence of Wnt, B-catenin, and cadherin pathways. Science. 303: 1483-1487.

Okuda H., Saitoh K., Hirai S., Iwai K., Takaki Y., Baba M., Minato N., Ohno S., Shuin T. 2001. The von Hippel-Lindau tumor suppressor protein mediated ubiquitination of activated atypical protein kinase C. J. Biol. Chem. 276: 43611-43617.

Otomo A., Hadano S., Okada T., Mizumara H., Kunita R., Nishijima H., ShowguchiMiyata J., Yanagisawa Y., Kohiki E., Suga E., Yasuda M., Osuga H., Nishimoto T., Narumiya S., Ikeda J. 2003. ALS2, a novel guanine nucleotide exchange factor for the 
small GTPase Rab5, is implicated in endosomal dynamics. Hum. Mol. Gen. 12(14): 1671-1687.

Pal A., Severin F., Lommer B. 2006. Huntingtin-HAP40 complex is a novel Rab5 effector that regulates early endosome motility and is up-regulated in Huntinton's disease. J. Cell Biol. 172: 605-618.

Parton R.G. \& Simons K. 2007. The multiple faces of caveolae. Nat. Rev. Mol. Cell Biol. 8: $185-194$.

Payne C.K., Jones S.A., Chen C., Zhuang X. 2007. Internalization and trafficking of cell surface proteoglycans and proteoglycan-binding ligands. Traffic. 8: 389-401.

Pelkmans L., Burli T., Zerial M., and Helenius A. 2004. Caveolin-stabilized membrane domains as multifunctional transport and sorting devices in endocytic membrane traffic. Cell. 118: 767-780.

Penengo L., Mapelli M., Murachelli A.G., Confalonieri S., Magri L., Musacchio A., Di Fiore P.P. 2006. Crystal structure of the ubiquitin binding domains of Rabex-5 reveals two modes of interaction with ubiquitin. Cell. 124: 1183-1195.

Raiborg C., Bache K.G., Mehlum A., Stang E., \& Stenmark H. 2001. Hrs recruits clathrin to early endosomes. EMBO J. 20: 5008-5021.

Raiborg C., Bache K.G., Gillooly D.J., Madshus I.H., Stang E., Stenmark H. 2002. Hrs sorts ubiquitinated proteins into clathrin-coated microdomains of early endosomes. Nat. Cell Biol. 4: 394-398.

Roth, T. F. \& Porter, K. R. 1964. Yolk protein uptake in the oocyte of the mosquito Aedes Aegypti. L. J. Cell Biol. 20: 313-332.

Royle S.J., Bright N.A., Lagnado L. 2005. Clathrin is required for the function of the mitotic spindle. Nature. 434: 1152-1157.

Saito K., Murai J., Kajiho H., Kontani K., Jurosu H., Katada T. 2002. A Novel binding protein composed of hemophilic tetramer exhibits unique properties for the small GTPase Rab5. J. Biol. Chem. 277(5): 3412-3418. 
Schenck A., Goto Silva L., Colinet C., Rhinn M., Giner A., Habermann B., Brnd M., Zerial M. 2008. The endosomal protein Appl1 mediates Akt substrate specificity and cell survival in vertebrate development. Cell. 133: 486-497.

Seabra M.C., Mules E.H., Hume A.N. 2002. Rab GTPases, intracellular traffic and disease. Tre. Mol. Med. 8: 23-30.

Shin D., Lee S. Y., Han S., Ren S., Kim S., Aikawa Y., Lee S. 2012. Differential polyubiquitin recognition by tandem ubiquitin binding domains of Rabex-5. Biochem. Biophys. Res. Commun. 423(4): 757-762.

Sigismund S., Woelk T., Puri C., Maspero E., Tacchetti C., Transidico P., Di Fiore P.P., Polo S. 2005. Clathrin-independent endocytosis of ubiquitinated cargos. Proc. Natl. Acad. Sci. USA. 102: 2760-2765.

Sigisimund S., Argenzio E., Tosoni D., Cavallaro E., Polo S., Di Fiore P.P. 2008. Clathrin-Mediated Internalization is essential for sustained EGFR signaling but dispensable for degradation. Dev. Cell. 15: 209-219.

Sorkin A. 2004. Cargo recognition during clathrin mediated endocytosis: a team effort. Curr. Opin. Cell. Biol. 16: 392-399.

Stenmark H. \& Olkonen V.M. 2001. The Rab GTPase family. Genome Biol. 2(5): REVIEWS3007.

Sung J.Y., Kim J., Paik S.R. 2001. Induction of neuronal cell death by Rab5A-dependent endocytosis of a-synclein. J. Biol. Chem. 276: 27441-27448.

Tall GG, Barbieri MA, Stahl PD, Horazdovsky BF, 2001. Ras-activated endocytosis is mediated by the Rab5 guanine nucleotide exchange activity of RIN1. Dev. Cell. 1: 73-82.

Tam, S.Y. et al. RabGEF1 is a negative regulator of mast cell activation and skin inflammation. Nat. Immunol. 5: 844-852.

Torres V.E., Sweeny W.E., Wang X., Qian Q., Harris P.C., Frost P., Avner E.D. 2003. EGF receptor tyrosine kinase inhibition attenuates development of PKD in Han:SPRD rats. Kidney International. 64: 1573-1579.

van Dam, E.M., and Stoorvogel W. 2002. Dynamin-dependent transferring receptor recycling by endosome-derived clathrin coated vesicles. Mol. Biol. Cell. 13(1): 169-182. 
Veithen A., Cupers P., Baudhuin P., Courtoy P.J. 1996. v-Src induces constitutive macropinocytosis in rat fibroblasts. J. Cell Sci. 109(8): 2005-2012.

Walker F., Abramowitz L., Benabderrahmane D., Duval X., Descatoire V., Hénin D., Lehy T., Aparicio T. 2009. Growth factor receptor expression in anal squamous lesions: modifications associated with oncogenic human papillomavirus and human immunodeficiency virus. Hum Pathol. 40(11): 1517-27.

Wang Y., Du D., Fang L., Yang G., Zhang C., Zeng R., Ulrich A., Lottspeich F., Chen Z. 2006. Tyrosine phosphorylated Par3 regulates epithelial tight junction assembly promoted by EGFR signaling. EMBO J. 25: 5058-5070.

Wells Alan. 1999. EGF Receptor. Intern. J. of Biochem. \& Cell Biol. 31: 637-643.

Woodworth C.D., Michael E., Marker D., Allen S., Smith L., Nees M. 2005. Inhibition of Epidermal Growth Factor Receptor increases expression of genes that stimulate inflammation, apoptosis and cell attachment. Mol. Cancer Ther. 4(4): 650-658.

Xu L., Lubkov V., Taylor L.J., Bar-Sagi D. 2010. Feedback regulation of Ras signaling by Rabex-5-Mediated Ubiquitination. Curr. Biol. 20: 1372-1377.

Yan H., Jahanshahi M., Horvath E.A., Liu H.Y., Pfleger C.M. 2010. Curr. Biol. 20: 1378-1382.

Yu L., Hiu-Chen F., Chen Y. 1999. Differential expression of Rab5a in human lung adenocarcinoma cells with different metastasis potential. Clin. Exp. Metastasis. 17: 213219.

Zerial M. \& McBride H. 2001, Rab proteins as membrane organizers. Nat. Rev. Mol. Cell Biol. 2: 107-117.

Zhang, W. 2008. Impaired brain cholesterol homeostasis and Alzheimer's disease. Trends in Neurosciences. Volume 5.

Zhenfeng Z., Stiegler A., Boggon T.J., Kobayashi S., Halmos B. 2010. EGFR-mutated lung cancer: a paradigm of molecular oncology. Oncotarget. 1(7): 497-514. 


\section{CHAPTER 2}

TYROSINE KINASE ACTIVITY OF RECEPTOR TYROSINE KINASES IS REQUIRED FOR ENDOSOME FUSION AND ACTIVATION OF RAB5 


\subsection{Abstract}

Activation of receptor tyrosine kinases is a key feature in receptor signaling and membrane trafficking processes. Here, we established that the receptor tyrosine kinase activity is required for fusion between early endosomes. AG1024, insulin receptor tyrosine kinase inhibitor, and AG1478, EGF receptor tyrosine kinase inhibitor, both blocked the in vitro endosome fusion in a concentration-dependent manner. We observed that Rab5: wild type partially rescued the fusion reaction, whereas a GTP hydrolysis defective mutant (Rab5: Q79L) mutant fully rescued it. We also observed that treatment of cells with either insulin receptor kinase inhibitor HNMPA-(AM) $)_{3}$, or EGF receptor tyrosine kinase inhibitor AG1478, blocked the formation of Rab5-positive endosomes as well as the activation of Rab5 in intact cells upon stimulation with insulin or EGF respectively. Furthermore, we also found that upon stimulation with EGF, an EGFreceptor catalytically inactive mutant failed to activate Rab5. Furthermore, both HNMPA-(AM) $)_{3}$ and AG1478 inhibitors also affected the endosomal co-localization of Rab5 and insulin receptor or EGF receptor respectively. However, the formation of Rab5: Q79L mutant-positive endosomes were not affected by either tyrosine kinase inhibitor. In addition, both HNMPA-(AM) $)_{3}$ and AG1478 inhibitors affected the association of Rin1 to membrane upon insulin stimulation. Furthermore, Rin1 did not fully support endosome fusion in the presence of the tyrosine kinase inhibitors. Consistent with these observations, Rin1 was unable to localize to membranes upon stimulation with ligand in presence of a tyrosine kinase inhibitor. These results constitute the first evidence that, at least in part, the enzymatic activity of tyrosine kinase receptors (either insulin or EGF receptor) is required for the fusion events via the activation of Rab5. 


\subsection{Introduction}

The early endosome is a key check-point in endocytic pathways, in which a decision is made to either be sorted to the late endosome/lysosome compartment or to be recycled back to the plasma membrane [Doherty and McMahon, 2009; Pfeffer, 2007]. Rab5 and its effectors, including EEA1, Rin1, Rabaptin-5, RAP6 and Rabex-5, together with other small GTPases (i.e., Rab 4, 7, 11, 15 and 22), are likely to tightly control the fusion and sorting of molecules that have entered the early endosome [Sorkin and von Zastrow, 2009; Scita and Di Fiore, 2010]. These homotypic and heterotypic vesicle fusions are regulated by several cytosolic and membranous factors. For example, the small GTPase Rab5 and its effectors regulate the fusion between early endosomes without affecting the fusion with late endosome or lysosomes. Thus, early endosome fusion is dependent on Rab5 proteins; it is symmetric and selective, thereby allowing orderly modification of ligand receptor interaction complexes and signaling in a sequential manner by altering the surroundings during receptor-ligand internalization [Bucci et al., 1992; Barbieri et al., 1994; Li et al., 1995; Barbieri et al., 1998; Barbieri et al., 2000; Brandhorst et al., 2006]. In addition, several Rab5-asociated proteins are also required for endosome-endosome fusion [Li et al., 1995; Horiuchi et al., 1997; Simonsen et al., 1998; Christoforidis et al., 1999; McBride et al., 1999; Nielsen et al., 2000; Lippe et al., 2001; Tall et al., 2001].

Endocytosis of the insulin receptor is initiated by the binding of its ligand [Russell et al., 1987; Khan et al., 1989; Liu and Roth, 1995; Di Guglielmo et al., 1998; Maggi et al., 1998]. The insulin receptor-ligand complex is then transported through the endocytic pathway, where it is then either recycled back to the cell surface or transported to late endosomes, and ultimately, the lysosome for degradation [Siemeister et al., 1995; Waters 
et al., 1995; Fucini et al., 1999]. The internalization of insulin receptors has been shown to be dependent on insulin receptor autophosphorylation, followed by the downstream phosphorylation and/or activation of insulin receptor substrate (IRS) or phosphatidylinositol 3 (PI3)-kinase through the clathrin-mediated pathway [Klein et al., 1987; Carpentier et al., 1992; Carpentier et al., 1993]. However, other reports have also suggested other pathways for insulin receptor internalization [Fan et al., 1982; Smith \& Jarett, 1990; Paccaud et al., 1992]. We have taken advantage of the well-characterized trafficking pathway of the activated insulin receptor [Fan et al., 1982; Klein et al., 1987], in order to measure fusion of insulin-insulin receptor vesicles (internalized Biotininsulin) with other endocytic vesicles that have been prepared by internalizing Avidin- $\beta$ galatosidase during fluid phase endocytosis in HepG2 cells. By allowing different populations of HepG2 cells to engage in receptor-mediated endocytosis of insulin linked to biotin and fluid phase endocytosis of Av- $\beta$-galatosidase, we are able to isolate donor and acceptor pools of endosomes, and examine for endosome fusion as previously developed cell-free endosome fusion assays [Braell, 1987; Gruenberg \& Howell, 1986; Mayorga et al., 1988; Wessling-Resnick \& Braell, 1990; Gorvel et al., 1991; Mullock and Luzio, 1992; Rubino et al., 2000].

Along the same lines, interaction of epidermal growth factor (EGF) [Citri \& Yarden, 2006] with EGF receptors leads to activation and tyrosine autophosphorylation of the receptor, which in turn creates binding sites for proteins containing Src homology 2 (SH2) and phosphotyrosine-binding domains (PTB) [O'Bryan et al., 1998]. Consequently, the activation of EGF-receptor dramatically accelerates endocytosis of EGF-receptor complexes through clathrin-coated pits [Huang \& Sokin, 2005]. Several 
factors affect the endocytosis of activated EGF-receptors, including Shc, Grb2, Cbl, Rab5, and PI3-kinase [O’Bryan et al., 1998; Burke et al., 2001; Huang \& Sorkin, 2005]. After internalization, EGF-receptors are efficiently sorted to the lysosomal compartment via early and late endosomes. Hence, increased internalization and lysosomal targeting, results in down-regulation of EGF-receptor and leads to attenuation of the growth factor signaling cascade [Burke et al., 2001]. In addition, several Rab5-asociated proteins are also required for endosome-endosome fusion [ $\mathrm{Li}$ et al., 1995; Horiuchi et al., 1997; Simonsen et al., 1998; McBride et al., 1999; Christoforidis et al., 1999; Nielsen et al., 2000; Lippe et al., 2001; Tall et al., 2001]. Given the importance of tyrosine phosphorylation in both signaling and trafficking of growth factors, specific and selective inhibitors of tyrosine kinase activity are important tools for studying EGF-receptor function [Burke et al., 2001; Sorkina et al., 2002; Citri et al., 2006; Doherty et al., 2009]. Therefore, the analysis of the effects of tyrosine kinase inhibitors on EGF-receptor trafficking and signaling may provide important information about the mechanisms by which the inhibitor(s) function(s) at the cellular level.

Here, we demonstrate that tyrosine kinase activity of two different RTKs (insulin and EGF-receptor) is required for the formation for enlarged Rab5-positive endosomes as well as for the activation of Rab5 in intact cells. We also observed that tyrosine kinase inhibitors (AG1024 and HNMPA-(AM) $)_{3}$ for insulin receptor and AG1478 for EGF receptor) blocked endosome fusion, whose inhibitory effect was linked to the activation of Rab5. Furthermore, we found that tyrosine kinase inhibitors of either receptor blocked the endosome fusion reaction stimulated by Rin1. More importantly, we have also observed that the addition of a constitutively active mutant of Rab5 (e.g., Rab5:Q79L), 
reversed the inhibitory effect of each inhibitor, suggesting a mechanism by which the tyrosine kinase activity of the receptor modulates early endosome fusion.

\subsection{Materials and Methods}

\subsubsection{Cell Culture and Materials}

HepG2 cells (American Type Culture Collection) were grown to confluence in Dulbecco's modified Eagle's medium supplemented with 5\% fetal bovine serum. NIH3T3-human insulin receptor (NIH-IR) cells, NR6 cells expressing the human EGFreceptor (NR6-E) and human EGF-receptor catalytically inactive mutant (K712M) (NR6K) were all grown in Dulbecco's modified Eagle's medium supplemented with 5\% fetal bovine serum containing $0.3-0.5 \mathrm{mg} / \mathrm{ml} \mathrm{G} 418$. Kinase inhibitors were purchased from EMD Biosciences (La Jolla, CA). EEA-1, Rab5, Rab11, P-Y100 and Rabex-5 antibodies were from Cell Signaling Technology (Beverly, MA). Rin1 antibodies were from Abcam Inc. Biotin-insulin and Avidin $\beta$-galactodidsase and the anti-insulin antibodies were purchased from Sigma-Aldrich (St. Louis, MO). All secondary antibodies were purchased from Jackson ImmunoResearch Laboratories (West Grove, PA). All other chemicals were obtained from Sigma unless otherwise stated.

\subsubsection{Endocytic Probes and Fractionation}

HepG2 cells were collected by centrifugation, washed three times in Hank's balanced salt solution (HBSS) with $15 \mathrm{mM}$ HEPES and supplemented with $1 \mathrm{mg} / \mathrm{ml}$ BSA (HBSSBSA, $\mathrm{pH}$ 7.0). Endocytosis of probes was performed with cells suspended in HBSS-BSA buffer. To prepare early endosomes loaded with Biotin-Insulin or Avidin- $\beta$-galatosidase,

cells $\left(8 \times 10^{8} / \mathrm{ml}\right)$ were allowed to internalize either $1 \mathrm{mg} / \mathrm{ml}$ Avidin- $\beta$-galatosidase or 
Biotin-Insulin $100 \mathrm{ng} / \mathrm{ml}$ for $5 \mathrm{~min}$ at $37^{\circ} \mathrm{C}$ in order to prepare the endosome fractions. The cells were then washed in uptake media at $800 \mathrm{x}$ g, followed by two more washes, once in HBSS-BSA buffer and once in homogenization buffer (HB, $0.25 \mathrm{M}$ sucrose, 20 mM HEPES, $0.5 \mathrm{mM}$ EGTA, pH 7.0). Cells were then resuspended in homogenization buffer $\left(4 \times 10^{8} / \mathrm{ml}\right)$ and passed 17 times through a stainless steel ball homogenizer. The homogenate was centrifuged at $800 \mathrm{x}$ g for $10 \mathrm{~min}$ to generate a post nuclear supernatant (PNS). Typical homogenization yielded $>80 \%$ of endocytosed marker in the PNS as determined by either radioactivity or immunoprecipitation of ligand. PNSs could then be used fresh for fractionation studies, cytosol preparation, or stored frozen in liquid nitrogen for later use (Gruenberg and Howell, 1986).

\subsubsection{Fusion Assay}

Two populations of vesicles containing Avidin- $\beta$-galatosidase and either BiotinInsulin or Biotin-EGF were mixed at $4^{\circ} \mathrm{C}$ to a final volume of $11 \mu \mathrm{l}$ in fusion buffer (250 mM Sucrose, $0.5 \mathrm{mM}$ EGTA, $1 \mathrm{mM}$ DTT, $1.5 \mathrm{mM} \mathrm{MgCl} 2,50 \mu \mathrm{g} / \mathrm{ml} \mathrm{B-BSA}, 50 \mathrm{mM}$ $\mathrm{KCl}$, and $20 \mathrm{mM}$ HEPES-KOH, $\mathrm{pH}$ 7.0). The buffer was supplemented with a regenerating system $(1 \mathrm{mM}$ ATP, $8 \mathrm{mM}$ creatine phosphate, 31 units $/ \mathrm{ml}$ creatine phosphokinase) or with a depleting system (5 $\mathrm{mM}$ mannose, 25 units $/ \mathrm{ml}$ hexokinase). AG1024 and AG1478 inhibitors as well as AG9, an inactive analog, were added to the reaction at the concentration indicated in each figure. After incubation at $37^{\circ} \mathrm{C}$ for 45 $\mathrm{min}$, the fusion reaction was stopped by cooling to $4^{\circ} \mathrm{C}$ (ice) for $10 \mathrm{~min}$. To measure the

immune complexes formed, the vesicles were solubilized by adding $150 \mu \mathrm{l}$ of solubilization buffer (1\% Triton X-100, $1 \mathrm{mM}$ EDTA, 0.1\% BSA, $0.15 \mathrm{M} \mathrm{NaCl}, 10 \mathrm{mM}$ 
Tris-HC1, $\mathrm{pH} 7.4$ ) containing $35 \mu \mathrm{g} / \mathrm{ml}$ Biotin-BSA. Biotin-BSA was used to quench any free Av-Gal due the presence of broken endosomes during the homozenization process. The solubilized samples were then added to wells that were plate coated with anti-insulin antibody. After a 60-min incubation period at $4^{\circ} \mathrm{C}$, immunoprecipitates were washed twice with $200 \mu \mathrm{l}$ of solubilization buffer and the $\beta$-galactosidase activity was measured by using 4-methylumbelliferyl-D-galactoside substrate [Mayorga et al., 1988]. There was a linear relationship between the amount of immunoprecipitate added to the enzymatic assay and the amount of product formed. Fusion-dependent immunoprecipitable enzymatic activity was expressed as a percent of the total immunoprecipitable activity in the assay. Total activity was determined by immunoprecipitating the immune complexes after mixing vesicles in the presence of detergent but in the absence of Biotin-BSA.

\subsubsection{Electron microscopy}

Colloidal gold particles of 10 and $20 \mathrm{~nm}$ were obtained from Sigma. Gold particles were coated with proteins (insulin and $\beta$-galactosidase) by standard techniques [Mayorga et al., 1989]. Binding and uptake of gold particles coated with the ligands were carried out under the similar conditions described by Mayorga et al., 1989 for soluble ligands into two separate populations of HepG2 cells by 5 -min incubated at $37^{\circ} \mathrm{C}$. The homogenization of cells and the fusion reaction were performed using the same protocols described above, except that both homogenization and fusion buffers were buffered with $30 \mathrm{mM}$ MES, pH: 6.6. They were centrifuged, and then the vesicles were mixed under fusogenic conditions. After the incubation, the fusion reaction was then stopped by adding $1 \%$ glutaraldehyde, which was prepared in HB buffer at room temperature. After 
$10 \mathrm{~min}$, samples were centrifuged for $15 \mathrm{~min}$ at $12,000 \mathrm{xg}$, washed and the samples were processed by transmission electron microscopy [Mayorga et al., 1989].

\subsubsection{Western Blot Analysis}

Lysate Preparation, SDS-PAGE, and Western Blotting: To prepare whole cell lysates, cell were washed twice with ice-cold PBS and lysed in ice-cold lysis buffer (20mM Tris$\mathrm{HCl} \mathrm{pH} 7.5,150 \mathrm{mM} \mathrm{NaCl}, 1 \%$ Triton X-100). The lysates were clarified by centrifugation, and protein concentrations were determined using the BCA Protein Assay Reagent Kit (Pierce). Proteins were resolved by SDS-PAGE and transferred to nitrocellulose membranes, which were blocked and probed with the indicated antibodies. To determine relative protein amounts, three representative exposures for each sample were quantified using National Institutes of Health ImageJ software.

\subsubsection{Confocal microscopy}

NR6-E, NR6-K or NIH3T3-human insulin receptor cells were seeded on glass coverslips at $1.0 \times 10^{5}$ per $3.8 \mathrm{~cm}^{2}$ well. The following day, cells were transfected using FugeneHD with either pEGFP-Rab5: WT or pEGFP-Rab5: Q79L. Cells were starved in serum-free Dulbecco's modified Eagle's medium supplemented with 5\% BSA Fraction V (MP Biomediclas, Solon, OH) for 14 hours, and treated as indicated with $100 \mu \mathrm{M}$ AG9, $100 \mu \mathrm{M}$ HNMPA-(AM) $)_{3}$ or $100 \mathrm{nM}$ AG1478 for 30 minutes at $4^{\circ} \mathrm{C}$, after which they were treated with either EGF or Insulin at $100 \mathrm{ng} / \mathrm{ml}$ for $1 \mathrm{hr}$ at $4^{\circ} \mathrm{C}$ and then stimulated to uptake the ligand for $5 \mathrm{~min}$ at $37^{\circ} \mathrm{C}$. They were washed with PBS and fixed in $4 \%$ paraformaldehyde for 20 minutes. Fixed cells were probed with rabbit polyclonal insulin receptor antibodies for $1 \mathrm{~h}$. Secondary antibodies used were Alexa594-conjugated goat 
anti-rabbit IgG. Coverslips were mounted with Prolong and viewed on a Leica TCS SP2 confocal microscope.

\subsubsection{Statistical analysis}

All experiments presented were repeated at least three times. The data represent the mean \pm SD calculated using Excel. Student's t-test was performed to calculate statistical significance.

\subsection{Results}

\subsubsection{Insulin receptor tyrosine kinase inhibitor blocks in vitro endosome fusion}

To prepare endosomes, $0.1 \mu \mathrm{g} / \mathrm{ml}$ Biotin-insulin and $2 \mathrm{mg} / \mathrm{ml}$ Avidin- $\beta$-Galactosidase were separately internalized for 5 min at $37^{\circ} \mathrm{C}$ in HepG2 cells as described in Materials and Methods. When the isolated vesicles containing Biotin-Insulin were mixed with vesicles containing Avidin- $\beta$-Galactosidase in an in vitro system in the presence of an ATP regenerating system at $37^{\circ} \mathrm{C}$, a cytosolic-dependent increase of complex formation was observed (Figure 5A). The effect of cytosol appeared to be saturable at about 1.0 $\mathrm{mg} / \mathrm{ml}$ protein and no fusion activity was observed without cytosol. As expected, in the presence of ATP-depleting system, very little fusion activity was observed (Figure 5A). Furthermore, the fusion was also time-dependent (Figure 6A).

To examine the specificity of the fusion assay, different populations of vesicles were employed. First, Biotin-insulin was internalized for $3 \mathrm{~min}$ at $37^{\circ} \mathrm{C}$. Second, Avidin- $\beta-$ Galactosidase was internalized by cells for $3 \mathrm{~min}$ and then chased for $0,3,6$, or 12 min to load the later compartments at $37^{\circ} \mathrm{C}$ (Figure 6B). Since the maximum fusion activity was observed when both probes were present at 3-min endosomes, we then examined fusion 
of such vesicles containing Biotin-insulin. As Avidin- $\beta$-Galactosidase was chased for an extended period of time, ATP-dependent fusion activity decreased rapidly (Figure 6B). After a 12 -min chase, fusion activity was reduced by $70 \%$ and no fusion activity was detected when Av-Gal was chased for 30 min (data not shown). These results indicate that fusion between Biotin-Insulin and Avidin- $\beta$-Galactosidase containing vesicles requires ATP, cytosolic proteins and is also time-dependent. The endosome reaction was affected by the ionic strength of the fusion buffer. The optimum concentration of $\mathrm{KCl}$ was $60 \mathrm{mM}$ as is the case in reconstitution studies with intracellular vesicular transport [Mayorga et al., 1989]. The in vitro endosome fusion assay showed similar requirements with other in vitro vesicle fusion systems (i.e., ATP, cytosol proteins, and salts). We also investigated the requirements of the small Rab5 in this in vitro assay since Rab5 is a critical regulator of the early endosome fusion [Bucci et al., 1992]. In Figure 6C, we show that the addition of cytosol depleted of Rab5 inhibited the endosome fusion. In contrast, the cytosol treated with control IgG or depleted of Rab11 and Rab4 did not affect the fusion reaction. Clearly our results indicate the specificity of the fusion reaction and more importantly, that this in vitro system is Rab5-dependent. We then confirmed the requirement of Rab5 in this in vitro system by observing that the addition of GDI to the in vitro system blocked the fusion reaction (Figure 6C). Thus, the requirement of Rab5 in this in vitro system raises the question whether Rab5 interacting proteins were also required in this endosome fusion system.

We examined the requirement of Rin1, a Rab5-guanine exchange factor, or other effectors (i.e., EEA1) in the fusion reaction. As shown in Figure 6C the depletion of Rin1 or EEA1 from the cytosol and the subsequent fusion reaction, showed an inhibitory 
effect. We also found that the addition of inactive fragment of Syntaxin 13 inhibited the fusion reaction (Figure 6C). These observations further confirmed that our in vitro fusion reaction was SNARE-dependent, as previously determined for the heterotypic fusion between clathrin coated vesicle and early endosomes, and homotypic fusion between early endosomes [Rubino et al., 2000]. Because PI3-kinase and NSF activities have been shown to regulate the fusion reaction, we investigated the role of these two activities in the fusion reaction by incubating endosomes in the presence of NEM (a NSF inhibitor), or Wortmannin (a PI3-kinase inhibitor). As shown in Figure 6C, the addition of $1 \mathrm{mM}$ NEM and $100 \mathrm{nM}$ Wortmannin strongly inhibited the fusion reaction. Taken together, these results indicate that the fusion between endosomes containing Biotin-insulin and Avidin- $\beta$-Galactosidase was mediated by a specific fusion event whose characteristics are similar to those described in other endosome-endosome fusion reactions [Gruenberg and Howell, 1986; Braell, 1987; Mayorga et al., 1988]. As described above, our data indicate that different activities (i.e., Rab5, Rin1, and PI3-K) are required in this novel fusion reaction between endosomes. Interestingly these activities have also been linked to the activated insulin receptor, including the formation of enlarged Rab5-positive endosomes in intact cells upon addition of insulin [Hunker et al., 2006b]. Therefore, we raised the question whether the tyrosine kinase activity of the insulin receptor was also required in the fusion between endosomes. To investigate this requirement, we first examined the effect of AG1024, an insulin receptor tyrosine kinase inhibitor (Parrizas et al., 1997) on the fusion reaction. As a control, we also examined the effect of an inactive tyrosine kinase inhibitor (AG9). When the fusion reaction was conducted in the presence of AG1024, we found a strong inhibition of the fusion reaction in a concentration dependent 
manner (Figure 5B). However, the addition of AG9 did not affect the fusion reaction, which suggests that this inhibitory effect was specific.

A similar protocol was followed to examine the endosome-endosome fusion morphologically. In these experiments, $20 \mathrm{~nm}$ colloidal gold coated with Biotin-insulin and $10 \mathrm{~nm}$ colloidal gold coated with Avidin- $\beta$-Galactosidase were localized in 5-min endosomes. These endosomes were incubated under standard fusogenic conditions and the vesicle pellets were then processed and observed by electron microscopy as described in Material and methods. As shown in Figure 7A, we observed vesicle fusion in the presence of cytosol at $37^{\circ} \mathrm{C}$ containing an ATP-regenerating system, evidenced by the presence of varying sized of gold particles within an endosome. However, we did not observe vesicle fusion when the vesicles were incubated in the presence of cytosol either at $4^{\circ} \mathrm{C}$ (Figure 7B) or in the presence of $20 \mu \mathrm{M}$ AG1024 kinase inhibitor (Figure 7C). We also determined the number of vesicles containing more than one size of gold particles within an endosome under several experimental conditions. After fusion reactions, we observed that $25 \pm 4 \%$ of the endosomes contained two different sizes of gold particles when the endosomes were incubated in the presence of cytosol supplemented with ATP (Figure 7D, line 2). However, in the absence of ATP at $37^{\circ} \mathrm{C}$ (Figure 7D line 1) or at $4^{\circ} \mathrm{C}$ (Figure 7D, line 4), or the addition of AG1024, we observed a strong inhibition of formation of endosomes containing two different sizes of gold particles (Figure 7D line 3). Because the fusion reaction required Rab5 and was also inhibited by AG1024, we tested whether AG1024 inhibitor would affect the endosome fusion stimulated by Rab5. Early endosome and cytosol preparations were pre-incubated with $20 \mu \mathrm{M}$ of AG1024 for 15 min prior to the initiation of the fusion reaction at $4^{\circ} \mathrm{C}$. It was seen that $\mathrm{AG} 1024$ 
partially inhibited early endosome fusion in the presence of Rab5: wild type. However, addition of Rab5: Q79L reversed the inhibitory effect of AG1024 inhibitor and stimulated endosome fusion (Figure 8). Therefore it is unlikely that the inhibition by AG1024 was due to nonspecific damaging of endosomal membranes.

Altogether, these observations may establish a potential role of the insulin receptor tyrosine kinase activity on the activation of the fusion reaction that is mediated by Rab5. Based on our initial observation, that Rab5:WT partially restored the fusion reaction affected by AG1024, and the fact that Rin1 stimulated endosome fusion, we then further examined the role of AG1024 inhibitor on the fusion reaction by investigating whether AG1024 inhibitor affects the endosome fusion stimulated by Rin1. In Figure 9, we showed that Rin1 stimulated endosome fusion in a concentration-dependent manner. However, Rin1 partially restored the inhibitory of AG1024. Taken together, these observations indicate that tyrosine kinase activity of insulin receptor plays, at least in part, an important role in Rin1-mediated endosome fusion.

\subsubsection{Insulin receptor inhibitor blocks fusion between endosomes in intact cells.}

Given the effect of AG1024 inhibitor on the in vitro Rab5-dependent fusion between endosomes, we examined whether the addition of insulin receptor tyrosine kinase inhibitor HNMPA-(AM) $)_{3}$ affects the changes in the Rab5 distribution and/or localization upon insulin stimulation in intact cells. HNMPA-(AM) $)_{3}$ is a cell-permeable and selective insulin receptor tyrosine kinase inhibitor analog of HNMPA [Saperstein et al., 1989]. For this purpose, we prepared cell lines expressing Rab5: wild type and Rab5: Q79L mutant, respectively. These cell lines were then treated $10 \mathrm{~min}$ with $100 \mathrm{ng} / \mathrm{ml}$ insulin in the 
absence or presence of $100 \mu \mathrm{M}$ HNMPA-(AM) $)_{3}$ inhibitor. After the incubation, cells were processed for immunofluorescence microscopy as described in Materials and Methods. As expected, in the absence of insulin, Rab5: wild type appears in diffuse and typically punctuate endosomal structures (Figure 10A-C). However, upon addition of insulin, Rab5: wild type was found in enlarged vesicles (Figure 10D-F). The sizes of these enlarged Rab5-positive endosomes were comparable to the endosomal size observed in cells expressing Rab5: Q79L mutant (compare Figure 10D-F with Figure $11 \mathrm{M}-\mathrm{X})$. Interestingly, in cells stimulated with insulin and treated with HNMPA-(AM) ${ }_{3}$ inhibitor, we observed a significant decrease in size of Rab5-positve endosomes in cells expressing Rab5: wild type (Figure 10J-L), but not in cells expressing Rab5: Q79L mutants (Figure 11VX). These observations are consistent with our in vitro endosome fusion data, in which the addition of Rab5: WT, but not Rab5: Q79L mutant, partially supported the fusion reaction in the presence of insulin receptor tyrosine kinase inhibitor (Figure 8). We also observed that insulin receptor co-localizes with Rab5 on endosomes when cells were stimulated with insulin in the absence of HNMPA-(AM) $)_{3}$ inhibitor (Figure 10D-F), but not in the presence of the inhibitor (compare Figure 10D-F and 10JL). Furthermore, analysis of Rab5: WT in cells stimulated with insulin in the presence of inhibitor showed a more diffuse (primary cytosolic) pattern, that is comparable with the distinct distribution of Rab5: wild type in the absence of insulin (compare Figure 10A-C and 10J-L). In contrast, in cells expressing Rab5: Q79L mutant, the addition of HNMPA$(A M)_{3}$ inhibitor failed to affect the formation of significantly enlarged Rab5:Q79L mutant-positive endosomes (compare Figure 11P-R and 11V-Y). Moreover, we observed that the internalized insulin receptor also co-localized with Rab5: Q79L mutant-positive 
endosomes, when cells were treated with HNMPA-(AM) $)_{3}$ inhibitor (Figure 11P-R and 11VY). These results suggest that the formation of these enlarged Rab5: Q79L-positive endosomes as well as the localization of insulin receptor were independent of the activation of the insulin receptor.

2.4.3 HNMPA-(AM) $)_{3}$ inhibitor blocks Rab5 activation and targeting of Rin1 to membrane upon insulin stimulation

To examine the role of insulin receptor on the activation of Rab5 upon stimulation of insulin, cells expressing either Rab5: WT or Rab5: Q79L mutant were incubated with or without HNMPA-(AM) $)_{3}$ inhibitor in the absence or presence of insulin. After incubation, Rab5 GTP-bound form was precipitated with GST-EEA1/N domain, as described in the Materials and Methods. As shown in Figure 12A, the addition of HNMPA-(AM) 3 inhibitor significantly diminished the amount of Rab5-GTP bound form when cells expressing Rab5: wild type were stimulated with insulin. However, in cells expressing Rab5: Q79L, the addition of HNMPA-(AM) $)_{3}$ inhibitor did not exhibit any effect at all (Figure 12B). Insulin receptor's activation leads to autophosphorylation, which is followed by the downstream phosphorylation of IRS [Fan et al., 1982; Russell et al., 1987] and association of molecules such as Grb2 [Liu and Roth, 1995] and Rin1 [Hunker et al., 2006a] through the clathrin mediated pathway. To further examine whether the insulin receptor kinase activity is linked to the membrane targeting to Rin1, the effect of HNMPA-(AM $)_{3}$ inhibitor was evaluated to determine whether this nucleotide exchange factor for Rab5 was targeted to membranes upon insulin stimulation. In Figure 13, we show that insulin receptor was tyrosine phosphorylated in the presence, but not in the absence, of insulin. Furthermore, insulin receptor was found associated to membranes, 
which indicates that the integrity of the isolated membrane fraction. IRS-1 and Grb2 were associated with membranes upon insulin stimulation. However, the addition of HNMPA$(\mathrm{AM})_{3}$ inhibitor partially blocked the membrane association of these molecules. Interestingly, Rin1 was also associated with membrane upon insulin stimulation, but not in the presence of HNMPA-(AM) $)_{3}$ inhibitor. We also examined the effect of HNMPA$(\mathrm{AM})_{3}$ inhibitor on the distribution of Rabex-5, another Rab5-GEF. As shown in Figure 13, Rabex-5 was recruited to the membrane upon stimulation with insulin. However, the addition of HNMPA-(AM) $)_{3}$ upon stimulation with insulin had no effect on the recruitment of Rabex-5 to the membrane. Taken together, these results are consistent with the observation that HNMPA-(AM) $)_{3}$ blocked the activation of Rab5 via Rin1 in intact cells.

2.4.4 EGF-receptor tyrosine kinase inhibitor blocks fusion between endosomes in intact cells

EGF-receptor activation leads to tyrosine autophosphorylation of the cytoplasmic tail and the subsequent recruitment of several factors including Shc, Grb2, PLC-c, Rin1 and Rabex-5 [Wells et al., 1999; Wiley 2003; Hayes et al., 2004]. To determine whether EGF-receptor kinase activity directly affects the activity of Rab5 in intact cells, we examined whether the addition of AG1478 inhibitor affects the changes in distribution and/or localization of Rab5 upon stimulation of EGF in intact cells. As expected, in the absence of EGF, Rab5:WT appears diffuse and typically in punctuate endosomal structures (see arrows in Figure 14C). However, upon addition of EGF, Rab5:WT was found in enlarged vesicles (see arrows in Figure 14F). In these cells, the enlarged Rab5positive endosomes were comparable in size to those endosomes observed in cells 
overexpressing Rab5:Q79L mutant (compare Figure 14F with Figure 15A-R). Furthermore, we observed a significant decrease in size of Rab5-positive endosomes in cells expressing Rab5:WT when treated with AG1478 (Figure 14L), but not in cells expressing the Rab5:Q79L mutant (Figure 15G-L). We also observed that in the absence of AG1478 inhibitor, EGF-receptor co-localizes with Rab5 on endosomes when cells are stimulated with EGF, but not in the presence of the inhibitor (compare Figure 14F and Figure 14L). However, in cells expressing the Rab5:Q79L mutant, addition of the AG1478 inhibitor failed to affect either the formation of enlarged Rab5-positive endosomes or the co-localization of internalized EGF-receptor with Rab5:Q79L mutantpositive endosomes, when cells were treated with the AG1478 inhibitor (Figure 15). In addition, we found that in the absence or in the presence of EGF, Rab5:WT appears diffuse and typically in punctate endosomal structures in cells expressing EGF-receptor catalytic inactive mutant (see arrows in Figure 14O-R). In contrast, we found enlarged Rab5-positive endosomes in cells expressing both Rab5:Q79L mutant and EGF-receptor catalytically inactive mutant (Figure 15O-R). As expected, EGF-receptor:WT, but not the catalytically inactive mutant of EGF-receptor, was tyrosine phosphorylated in the presence of EGF in these cells (Figure 16). These observations are consistent with the idea that the EGF-receptor kinase activity is required for formation of enlarged Rab5positive endosomes as well as the subsequent co-localization for Rab5 and EGF-receptor on the endosomes. Thus, it is possible to speculate that the AG418 inhibitor should alter the size distribution of endosomes in cells expressing Rab5:WT, but not in cell expressing Rab5:Q79L mutant. To test this hypothesis, we expressed GFP-Rab5:Q79L and GFPRab5:WT proteins respectively in NR6 cells. Rab5-positive endosomes were 
identified by confocal immunofluorescence microscopy and then quantified using the NIH Image J software. We observed that in NR6-E cells expressing Rab5:WT, the endosomes were generally smaller with an average perimeter of $1.51 \mu \mathrm{m}$ (or $0.48 \mu \mathrm{m}$ in diameter) and a relative variance of 1.70 In contrast upon stimulation with EGF, Rab5positive endosomes were enlarged with an average perimeter of $3.29 \mu \mathrm{m}$ (or $1.05 \mu \mathrm{m}$ in diameter) and a relative variance of 1.41 (Figure 17A).We also observed that the addition of AG418 inhibitor to NR6-E cells expressing Rab5:WT, which were then stimulated with EGF, clearly decreased size of the endosomes (average perimeter of $1.69 \mu \mathrm{m}$ [or $0.54 \mu \mathrm{m}$ in diameter] and a relative variance of 1.61) (Figure 17B). As expected, the addition of AG9 an inactive analog, did not affect neither endosome size nor endosome distribution (Figure 17A, B). These observations are in agreement with the inhibitory effect of AG418 on the activation of Rab5 in NR6-E cells upon stimulation of EGF. Importantly, we observed that in NR6-E cells expressing Rab5: Q79L the size distribution of the endosomes showed an average perimeter of $5.74 \mu \mathrm{m}$ (or $1.83 \mu \mathrm{m}$ in diameter) and a relative variance of 1.44 and were not affected by the addition of either EGF or AG418 inhibitor (Figure 17C). These observations are consistent with the fact that the amount of GTP-bound form of Rab5 was not affected in NR6-E cells expressing Rab5:Q79L mutant.

Lastly, we observed that in NR6-K cells expressing Rab5:Q79L, the size distribution of the endosomes were comparable to those observed in NR6-E cells expressing Rab5:Q79L (Figure 17D). Furthermore, when EGF was added to NR6-K cells expressing Rab5:WT, Rab5-positive endosomes were not enlarged (average perimeter of $1.60 \mu \mathrm{m}$ [or $0.51 \mu \mathrm{m}$ in diameter], and a relative variance of 1.71) (Figure 17D). Taken together, 
this quantitative difference between Rab5:WT either in the absence or in the presence of EGF, are significant, especially reflected by the extended tail of Rab5:WT endosome distribution in cells stimulated with EGF.

\subsubsection{AG1478 inhibitor blocks the activation of Rab5 in intact cells}

To further examine the role of EGF-receptor kinase activity on the activation of Rab5 upon stimulation with EGF, cells expressing either Rab5:wild type or Rab5:Q79L mutant were incubated with or without the AG1478 inhibitor in the absence or presence of EGF. After incubation, active or GTP-bound form of Rab5 was precipitated with GST-EEA1 domain as described in Materials and methods. As shown in Figure 18A, the addition of AG1478 inhibitor significantly diminished the amount of GTP-bound form of Rab5 when the cells expressing both Rab5:WT and EGF-receptor: wild type were stimulated with EGF. However, addition of the AG1478 inhibitor to cells expressing the Rab5:Q79L mutant had no effect at all (Figure 18B). Furthermore, we also found that in cells expressing EGF-receptor catalytically inactive mutant and Rab5:WT, Rab5 was not activated and the catalytically inactive mutant of EGF-receptor was not tyrosine phosphorylated upon addition of EGF (Figure 18A and B). These data demonstrate that the inhibition of the EGF-receptor activity is linked to the inactivation of Rab5.

\subsubsection{EGF-receptor tyrosine kinase inhibitor blocks the recruitment of Rin in intact cells}

Given the effect of AG1478 inhibitor on the activation of Rab5 in intact cells, we examined whether the addition of AG1478 inhibitor can affect the changes in membrane distribution of several factors associated with EGF-receptor:WT, upon stimulation with EGF in intact cells. As expected, either in the absence or presence of EGF, EGF-receptor was always found in the membrane fraction (Figure 19A). EGF-receptor was also 
tyrosine phosphorylated only in the presence of EGF, while the addition of the AG1478 inhibitor blocked the phosphorylation of EGF-receptor (Figure 19A). Consistent with this data, in the absence (but not in presence) of AG1478 inhibitor, Shc and Grb2 were recruited to the membrane fraction upon stimulation with EGF (Figure 19B and C). Furthermore, Rin1 was recruited to the membrane upon EGF stimulation. In contrast, Rin1 was poorly localized on the membrane in the presence of AG1478 and upon stimulation with EGF (Figure 19D). Interestingly, Rin1 was also tyrosine phopshorylated upon EGF stimulation (Figure 20), but not in the presence of AG1478 inhibitor and in cells expressing EGF-receptor catalytically inactive mutant. As an extension of our observation, we also investigated the effect of AG1478 inhibitor on the membrane distribution of Rabex-5. As shown in Figure 3E, Rabex-5 was recruited to the membrane upon stimulation with EGF. However, the addition of AG1478 upon stimulation with EGF had no effect on the recruitment of Rabex-5 to the membrane. These data demonstrate that in addition to being able to block the recruitment of Shc and Grb2 factors, the inhibition of the tyrosine kinase activity of EGF-receptor is linked to a selective membrane targeting of Rin1. Consistent with these observations we found that Rin1, Rabex-5 and She were not translocated to membranes upon EGF stimulation in cells expressing EGF-receptor catalytically inactive mutant (Figure 20). As expected, EGF receptor catalytically inactive mutant was not tyrosine phosphorylated upon addition of EGF (Figure 21). Taken together, these observations suggest that both Rin1 and Rabex-5 are targeted to membrane upon EGF stimulation but they are not translocated either in cells expressing EGF-receptor catalytically inactive mutant or selectively affected in the presence of AG1478 inhibitor. 


\subsubsection{EGF-receptor tyrosine kinase inhibitor inhibits in vitro endosome fusion}

We have previously demonstrated that different factors (i.e., Rab5, Rin1 and PI3kinase) were required for the fusion reaction between endosomes containing EGFreceptor [Tall et al., 2001]. Interestingly, these activities have also been linked to the activated EGF-receptor, which in turn involve the formation of enlarged Rab5-positive endosomes in intact cells upon stimulation with EGF [Barbieri et al., 2000]. Therefore, we raised the question of whether tyrosine kinase activity of the EGF-receptor is also required in the fusion between endosomes. To investigate this requirement, we first examined the effect of AG1478 inhibitor [Yamamoto et al., 2006; Kondratov et al., 2010] on the fusion reaction. As a control, we also examined the effect of an inactive tyrosine kinase inhibitor (AG9). Five-min vesicles containing either Biotin (B)-EGF or Avidin(Av)-GAL were mixed in fusion buffer supplemented with $1 \mathrm{mg} / \mathrm{ml}$ of cytosol containing an ATP-regenerating system either in the absence or in the presence of different concentrations of AG9 and AG1478 (Figure 22A). Samples were then transferred to $37^{\circ} \mathrm{C}$ for the indicated times and processed as described in Materials and methods to determine the percentage forming the immune complex. When the fusion reaction was conducted in the presence of AG1478, we observed a strong inhibition of the fusion reaction that occurred in a concentration dependent manner. However, the addition of AG9 did not affect the fusion reaction in comparison to the control, which argues for the specificity of the exhibited inhibitory effect (Figure 22A).

Because of the inhibitory pattern of AG1478 exhibited in the fusion reaction (Figure 4A) and because Rab5 is a key factor required for fusion between endosomes directed by the EGF-receptor [Bucci et al., 1992; Barbieri et al., 1994], we tested whether the 
AG1478 inhibitor would affect endosome fusion stimulated Rab5. Early endosome and cytosol preparation were pre-incubated with $20 \mu \mathrm{M}$ AG1478 for 15 min prior to the initiation of the fusion reaction. The addition of AG1478 inhibited early endosome fusion in the presence of Rab5:WT, however, the addition of Rab5:Q79L mutant reversed the inhibitory effect and thus stimulated endosome fusion (Figure 22B). On the basis of previous data [Tall et al., 2001; Hunker et al., 2006] and the results reported here, we then investigated the effect of the tyrosine kinase enzymatic activity of the EGF-receptor on the endosome fusion stimulated by Rin1. Rin1 is a guanine nucleotide exchange factor for Rab5 that supports endosome fusion [Tall et al., 2001; Galvis et al., 2009]. As shown in Figure 23, Rin1 stimulated endosome fusion. As expected, the addition of AG9 did not affect the endosome fusion. However, AG1478 inhibitor partially blocked endosome fusion stimulated by Rin1. Collectively, these results reinforce the conclusion that tyrosine kinase activity of EGF-receptor may be involved in the activation of Rab5, at least in part, via Rin1.

\subsection{Discussion}

Given the importance of tyrosine phosphorylation, which is produced upon stimulation with growth factors in intact cells, specific and selective inhibitors of tyrosine kinase activity are an important means for investigating receptor tyrosine kinases (i.e., EGF-receptor) [Citri \& Yarden, 2006]. Therefore, the utilization of these inhibitors may be critical for elucidation of the role that tyrosine kinases play in membrane trafficking and signaling [Burke et al., 2001]. Furthermore, the activation of intrinsic or associated tyrosine kinases during the internalization of growth factor receptors is a key feature of 
both endocytic and signaling processes. Indeed, the studies on endocytosis of kinaseinactive mutants of several growth factors, including the EGF-receptor, suggested that kinase activity is necessary for maximal internalization rate, endosomal localization and down regulation of the receptor [Wells et al., 1999; Sorkina et al., 2002; Barbieri et al., 2003; Wiley 2003].

We have shown that the addition of HNMPA-(AM) $)_{3}$ tyrosine kinase inhibitor blocked the formation of enlarged Rab5-positive endosomes upon insulin stimulation in intact cells expressing Rab5:wild type, but not Rab5:Q79L mutant. Consistent with this observation, the receptor tyrosine kinase inhibitors (i.e., AG1024) specifically block the fusion between early endosomes. Interestingly, the inhibitory effect was reversed by the addition of Rab5: Q79L mutant. These data suggest a positive role of receptor tyrosine kinase activity on endosome fusion that is mediated by the activation of Rab5, which is consistent with increase in endosome size observed upon ligand stimulation.

The insulin receptor is a well-characterized system, which involves the internalization of the receptor from the plasma membrane to endosome upon ligand stimulation. In our experimental conditions, the binding of Biotin-insulin was saturated and nonspecific binding, measured in the presence of 300 fold of unlabeled Insulin, was less than $3.2 \%$ of the total counts/min in the pellet. By Scatchard analysis, we determined dissociation constants for Biotin-insulin $(\mathrm{Kd}=0.33 \mathrm{nM})$ and for unmodified insulin $(\mathrm{Kd}=0.29 \mathrm{nM})$ in HepG2 cells and these values were comparable to values reported for unmodified insulin in the order of the nM [Backer et al., 1991; Scholz et al., 1992]. When these two endocytic vesicles containing Biotin-insulin and Avidin- $\beta$-Galactosidse are brought together upon vesicle fusion, the resulting enzymatic activity of $\beta$-galactosidase in the 
complex, points to the specificity of the fusion reaction (Figures 5 and 6). The results reported here show that the fusion reaction is time, ATP and cytosol dependent with an optimal temperature of $37^{\circ} \mathrm{C}$.

Morphological analysis of the fusion events clearly shows that in the presence of cytosol the formation of endosome containing different size of gold particles. Consistent with these observations, fluorescence microscope analysis revealed that the accumulation of insulin receptor in early endosomes is dependent on tyrosine kinase activity since the insulin receptor is localized on the plasma membrane by the addition of the HNMPA$(\mathrm{AM})_{3}$ inhibitor (Figure 10). Interestingly, we also observed that the presence of HNMPA-(AM) $)_{3}$ inhibitor blocked the formation of enlarged Rab5-positve endosomes (Figure 10) as well as the activation of Rab5 in intact cells (Figure 12). However, the addition of HNMPA-(AM) $)_{3}$ inhibitor neither blocked the intracellular localization of insulin receptor nor the formation of enlarged Rab5-positve endosomes in cells expressing Rab5: Q79L mutant.

Interestingly, we also observed that the presence of AG1024 inhibitor blocked the activation of Rab5: wild type, but not the activation of Rab5: Q79L mutant, in intact cells (Figure 12). Thus, these data establish that tyrosine kinase activity of the insulin receptor is, in part, responsible for the formation of enlarged Rab5-positive endosomes, which is linked to the activation of Rab5. The potential mechanism of this inhibitory effect may be associated with Rin1, since it is associated with the insulin receptor upon ligand stimulation [Hunker et al., 2006a], and because it is also required for the fusion assay, whose fusogeneic activity is partially reversed by AG1024 inhibitor. These observations raise the possibility that the tyrosine kinase enzymatic activity of the insulin receptor may 
be required during the fusion assay. This inhibitory effect was concentration-dependent and specific since AG9 (an inactive analog) did not affect the fusion reaction. More importantly, the presence of this inhibitory effect may be associated with an effect on the interaction between Rin1 and activated insulin receptor. This is in strong agreement with the fact that Rin1 was found significantly less associated with membrane fraction in the presence of HNMPA-(AM) $)_{3}$ upon insulin stimulation (Figure 13). However, the association of Rabex-5 (another Rab5 exchange factor) with membranes was not affected in the presence of inhibitor. These observations indicate that this inhibition by HNMPA$(\mathrm{AM})_{3}$ may be selectively associated with Rin1, but not with Rabex-5.

Because stimulation of the fusion reaction by Rin1 and Rab5, but not Rab5: Q79L mutant, were also partially blocked by AG1024 raises the possibility that once Rab5 is already activated by its exchange factor, part of this in vitro endosome fusion may be independent of AG1024 inhibitor. Alternatively, it is also possible that AG1024 does not completely block insulin receptor tyrosine kinase enzymatic activity in our in vitro system, which, in part, may also contribute the inability of the AG1024 to fully block the fusion reaction. Nevertheless, our in vitro and in vivo data support that the tyrosine kinase enzymatic activity of the insulin-receptor is required during the endosome fusion assay.

On the other hand, we have also demonstrated that AG1478, an EGF-receptor tyrosine kinase inhibitor, blocked the fusion between endosomes. This inhibitory effect was reversed by the addition of Rab5:Q79L mutant, but not by the addition of Rab5:WT. Consistent with this observation, AG1478 blocked the formation of enlarged Rab5positive endosomes upon EGF stimulation in intact cells. This observation is further supported by the significant reduction in the perimeter of Rab5-positive endosomes 
(Figure 17). Thus, these data suggest a positive role of receptor tyrosine kinase activity on endosome fusion that is mediated by the activation of Rab5, which is in agreement with an increase in early endosome size observed upon EGF stimulation. Interestingly, we also observed that the presence of AG1478 inhibitor blocked the activation of Rab5 in intact cells expressing Rab5:WT (Figure 18). However, the addition of AG1478 inhibitor neither blocked the intracellular localization of EGF-receptor nor the formation of enlarged Rab5-positve endosomes in cells expressing Rab5:Q79L mutant (Figure 15). These results are supported by the observation that the perimeter of Rab5-positive endosomes in cells expressing Rab5:Q79L mutant were not significantly affected by the addition of either EGF or AG418 inhibitor (Figure 17). Thus, these data establish that tyrosine kinase activity of the EGF-receptor is required for the formation of enlarged Rab5-positive endosomes, and more importantly, they also demonstrate that the inhibition of the tyrosine kinase activity of the EGF-receptor is linked to the inactivation of Rab5. Fluorescence microscopy analysis also revealed that the accumulation of EGFreceptor in early endosomes is dependent on tyrosine kinase activity, since AG1478 inhibitor also blocked the internalization of the EGF-receptor (compare Figure 14E and $\mathrm{H})$.

Similarly, it has been demonstrated that PD158780, another EGF receptor tyrosine kinase inhibitor, also affected early steps of internalization [Sorkina et al., 2002; Wolf et al., 2011]. Thus, it is possible that these inhibitors may work at different steps during endocytosis of EGF-receptor by affecting the recruitment of selective factors [Thomas et al., 2003; Kondratov et al., 2010]. PD158780 prevented the targeting of EGF-receptor into coated pits by altering the recruitment of subunits of the AP2 complex [Sorkina et 
al., 2002]. However, here we showed that Rin1, a Rab5-GEF, was not recruited to the membrane upon EGF stimulation in the presence of AG1478. The possible mechanism of this inhibitory effect may be associated with the fact that Rin1 is associated with EGFreceptor upon ligand stimulation [Barbieri et al., 2003], and since Rin1 is also required for the fusion assay [Tall et al., 2001; Galvis et al., 2009], it raises the possibility that the tyrosine enzymatic activity of the EGF-receptor may be required during the fusion assay. To our surprise, we found that the addition of AG1478 inhibitor blocked the fusion reaction. This inhibitory effect was concentration-dependent and specific, since an inactive analog (i.e., AG9) did not affect the fusion reaction. More importantly, stimulation of the fusion reaction by Rin1 was also partially blocked by AG1478, suggesting that the presence of AG1478 may affect the recruitment of Rin1 to membrane, which is in strong agreement with the fact that Rin1 was found to be significantly less associated with membrane fraction in the presence of AG1478 upon EGF stimulation. Consistent with this idea, we also found that Rab5:WT partially reversed the inhibitory effect of AG1478. Alternatively, it is possible that Rin1 may also activate Rab5 independently of its association with the activated EGF-receptor in our in vitro endosome fusion system. Therefore, once Rab5 is activated, it will increase the endosome fusion independently of the presence AG1478 inhibitor, which is consistent with the observation that Rin1 activates Rab5 in an in vitro system [Tall et al., 2001]. In support of this idea, the addition of AG1478 neither affects the endosome fusion stimulated by Rab5:Q79L mutant nor the formation of enlarged Rab5:Q79L mutant-positive endosomes in intact cells. Nevertheless, our in vitro and in vivo data support the idea that the tyrosine kinase 
enzymatic activity of the EGF-receptor, at least in part, is required during the fusion assay.

Another view of the fusion reaction between endosomes containing Biotin-insulin or Biotin-EGF and Avidin- $\beta$-Galactosidase is that AG1024 inhibitor may affect the activation of Rab5 in one set of endosomes containing insulin receptor (i.e., Biotininsulin or Biotin-EGF endosomes) but not in the other set of endosomes containing Avidin- $\beta$-Galactosidase, a fluid phase marker. Therefore, this model predicts that Rab5 should be present and active in both sets of endosomes during the fusion reaction. Our results are in strong agreement with previous observations that Rab5 was required for the fusion in both endosomes [Barbieri et al., 1998]. However, we have found that Rab5 is activated during the enodocytosis of several fluid phase markers (i.e., HRP and $\beta$ Galactosidase). Specifically, we observed that activation of Rab5 during the uptake of fluid phase marker is less robust $(\sim 10 \%)$ as compared with the activation of Rab5 during either EGF or insulin stimulation (Figures $24 \& 25$ ), which may help to explain, in part, the fact that this endosome fusion is not completely inhibited in the presence of a tyrosine kinase inhibitor (Figure 5). These observations are consistent with previous observations where Rab5 was localized in endosomes containing HRP as a fluid phase marker markers [Gruenberg \& Howell, 1986; Chavrier et al., 1990; Mu et al., 1995; Singh et al., 2003; Hunker at al., 2006; Zhu et al., 2007; Zhu et al., 2009]. Interestingly, it has also been recently demonstrated that active Rab5 was found on macropinosomes [Feliciano, et al., 2011]. Thus, it is possible that several mechanisms of activation of Rab5 are taking place during receptor-mediated endocytosis and endosome fusion. For instance, dependent on cell type [Han \& Colicelli, 1995], overexpression of Rabex-5 induces the formation of 
enlarged endosome [Horiuchi et al., 1997; Zhu et al., 2009], but not the overexpression of either Rin1 [Semerdjieva et al., 2008] or RAP6 in mammalian cells; Rin1 requires tyrosine phosphorylation of the EGF-receptor [31], while Rabex-5 requires ubiquitination [Mattera et al., 2006; Penengo et al., 2006] and RAP6 does not interact with EGFreceptor directly [Su et al., 2007]. These Rab5-GEFs were also required in several in vitro endosome fusion reactions [Horiuchi et al., 1997; Tall et al., 2001; Hunker et al., 2006]. Thus, it is possible that several mechanisms of activation of Rab5 are taking place during EGF-receptor endocytosis and endosome fusion. Given the importance of tyrosine phosphorylation in signaling and endocytosis by hormones and growth factors, specific and selective inhibitors of tyrosine kinase activity are important means for investigating receptor tyrosine kinases biological functions. Thus, the utilization of these inhibitors may be critical for elucidation of the role of tyrosine kinase activity for both in vitro and in vivo setting. Furthermore, the activation of intrinsic or associated tyrosine kinases during the internalization of growth factor receptor is a key feature of endocytosis and signaling processes, in which several small GTP-ases have been implicated, including Rab5.

In conclusion, we have used in vivo and in vitro approaches to investigate the role of tyrosine phosphorylation during the fusion reaction. Specifically, we have demonstrated that tyrosine kinase inhibitors affect in vitro endosome fusion, the formation of enlarged Rab5-positive endosomes and activation of Rab5 in intact cells. These data suggest that the receptor kinase activity may provide a link between early endosomes and signaling molecules, which in turn, will activate the small GTPase Rab5 as well as directly increase the fusion activity. 

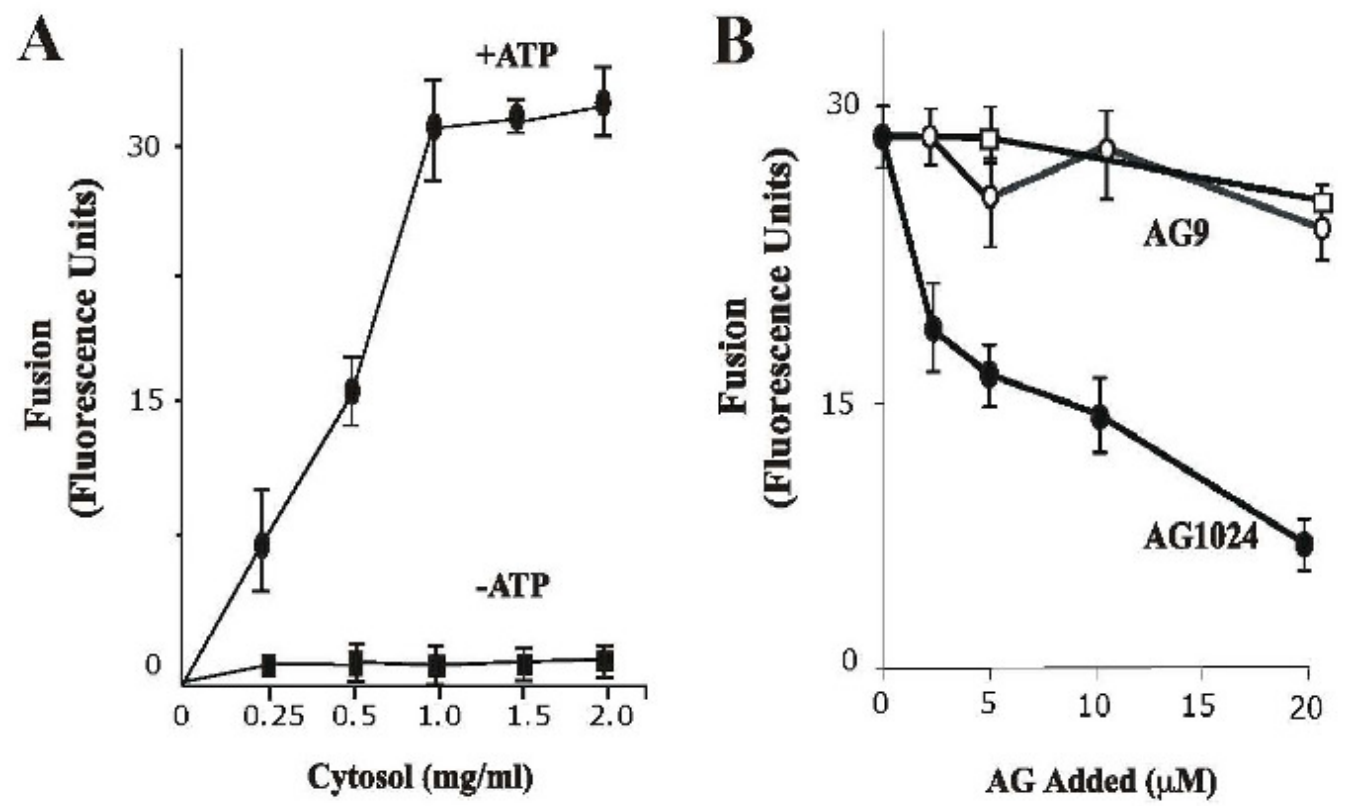

Figure 5. Effect of insulin receptor kinase inhibitor on fusion between endosomes. (A) Effect of cytosol and energy on the endosome-endosome fusion. Five-min vesicles containing either Biotin-Insulin (B-Ins) or Avidin- $\beta$-Galactosidase (Av-GAL) were mixed in fusion buffer supplemented with different amount of cytosol containing ATPregenerated $(\bullet)$ or ATP depleted systems $(\boldsymbol{\bullet})$. Samples were then transferred to $37^{\circ} \mathrm{C}$ for the indicated times and processed as described in Material and Methods to determine the percentage forming the immune complex formation. The data are presented as means \pm SD of four independent experiments. (B) Fusion assay was performed under standard conditions as described above either in the absence)( or in the presence of different amounts of AG9 q) and AG102 $4(\bullet)$ containing $1 \mathrm{mg} / \mathrm{ml}$ of cytosol. The data are presented as means $\pm \mathrm{SD}$ of four independent experiments. 

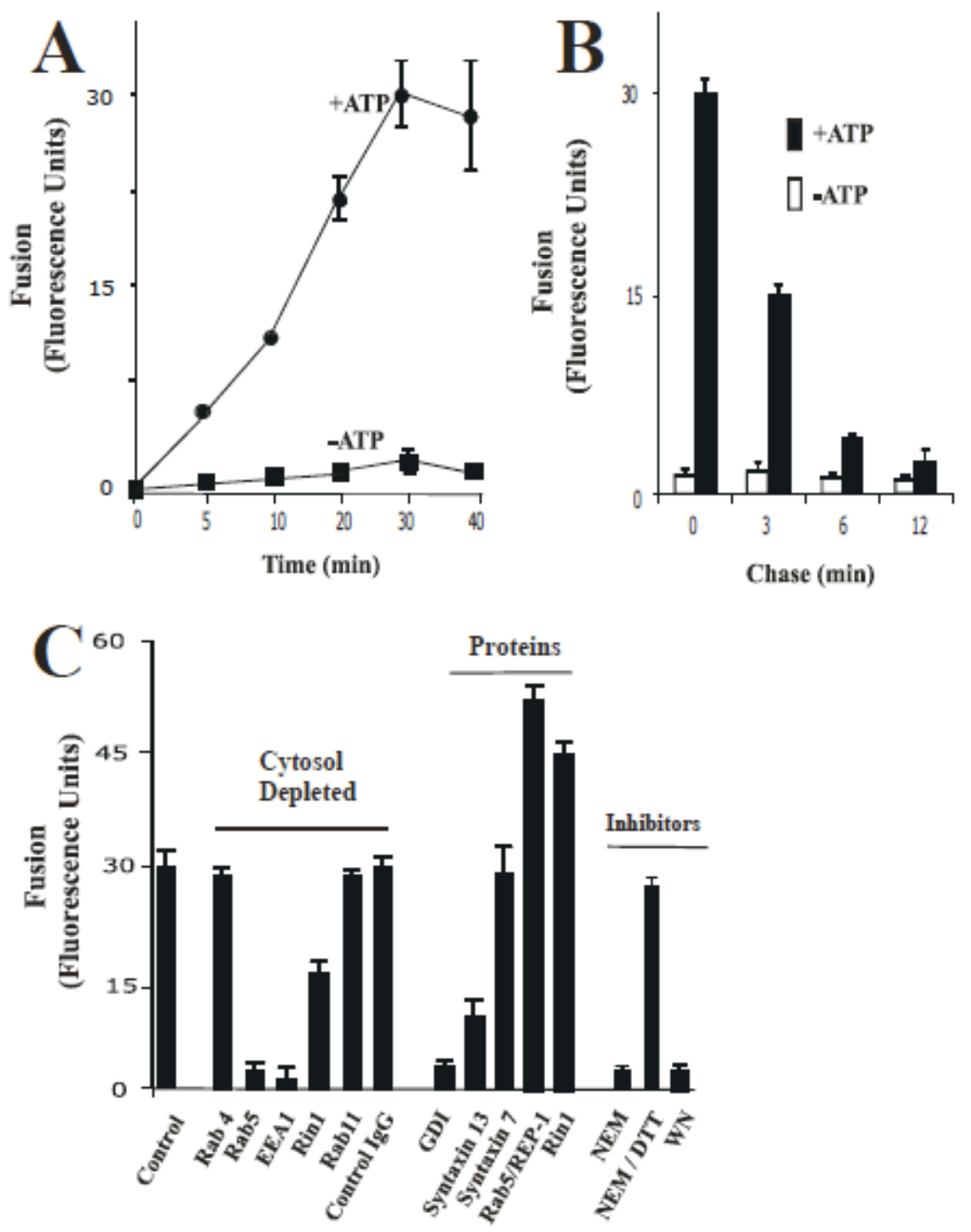

Figure 6. Fusion between endosomes. (A) Effect of time on the endosome-endosome fusion. Two sets of vesicles prepared from cells that have been allowed to endocytose BIns and $\mathrm{Av}-\mathrm{GAL}$ for $5 \mathrm{~min}$ at $37^{\circ} \mathrm{C}$, respectively. Then the vesicles were mixed in fusion buffer supplemented with different amounts of cytosolic proteins $/ \mathrm{ml}$ containing ATPregenerated $(\bullet)$ or ATP-depleted systems ( $\bullet$ ). Samples were then transferred to $37^{\circ} \mathrm{C}$ for the indicated times and processed as described in Material and Methods to determine the percentage immune complex formation. The data are presented as means $\pm \mathrm{SD}$ of four independent experiments. (B) Av-GAL was internalized by a 3-min uptake at $37^{\circ} \mathrm{C}$ and chased for $0,3,6,12 \mathrm{~min}$. Cells were then incubated at $4^{\circ} \mathrm{C}$ for $1 \mathrm{~h}$ with B-Ins. Endocytosis of Insulin was induced by incubation at $37^{\circ} \mathrm{C}$ and the amount of B-Ins associated with Av-GAL was quantitated at each time by solubilizing the cells in the presence of excess of biotin-BSA. Results are expressed as fluorescence units, which 
indicates the percentage of the immune complex formed after solubilization of vesicles in the absence of biotin-BSA. (C) Vesicles fractions were obtained and resuspended in homogenization and treated as following: Antibodies: $200 \mathrm{ng} / \mathrm{ml}$ control IgG, or antiRab4, anti-Rab5, or anti-EEA1, or anti-Rab11, or anti-Rin1 antibodies were added to fusion reaction; GDI proteins: vesicles were incubated in the presence of $1 \mathrm{uM} \mathrm{GDI}$, washed and then resuspended in fusion buffer; Rab5 proteins: $0.3 \mu \mathrm{g} / \mathrm{ml}$ Rab5-REP-1 complex was added to the fusion reaction; Rin 1 proteins: $0.2 \mu \mathrm{g} / \mathrm{ml}$ of Rin1 was added to the fusion reaction; NEM treatment: Cytosol and vesicles were pretreated with $0.75 \mathrm{mM}$ $\mathrm{NEM}$ at $4^{\circ} \mathrm{C}$ for $45 \mathrm{~min}$ and excess of NEM was inactivated by $2 \mathrm{mM}$ DTT. Fusion was carried out in the presence of ATPregenerating system, and the ATP-dependent fusion was expressed as a fraction of the fusion obtained with non-treated vesicles resuspended in $1.5 \mathrm{mg}$ of normal cytsosol $/ \mathrm{ml}$ for $45 \mathrm{~min}$ at $37^{\circ} \mathrm{C}$. Background was measured by incubation for $45 \mathrm{~min}$ at $4^{\circ} \mathrm{C}$. The data are presented as means $\pm \mathrm{SD}$ of three independent experiments. 

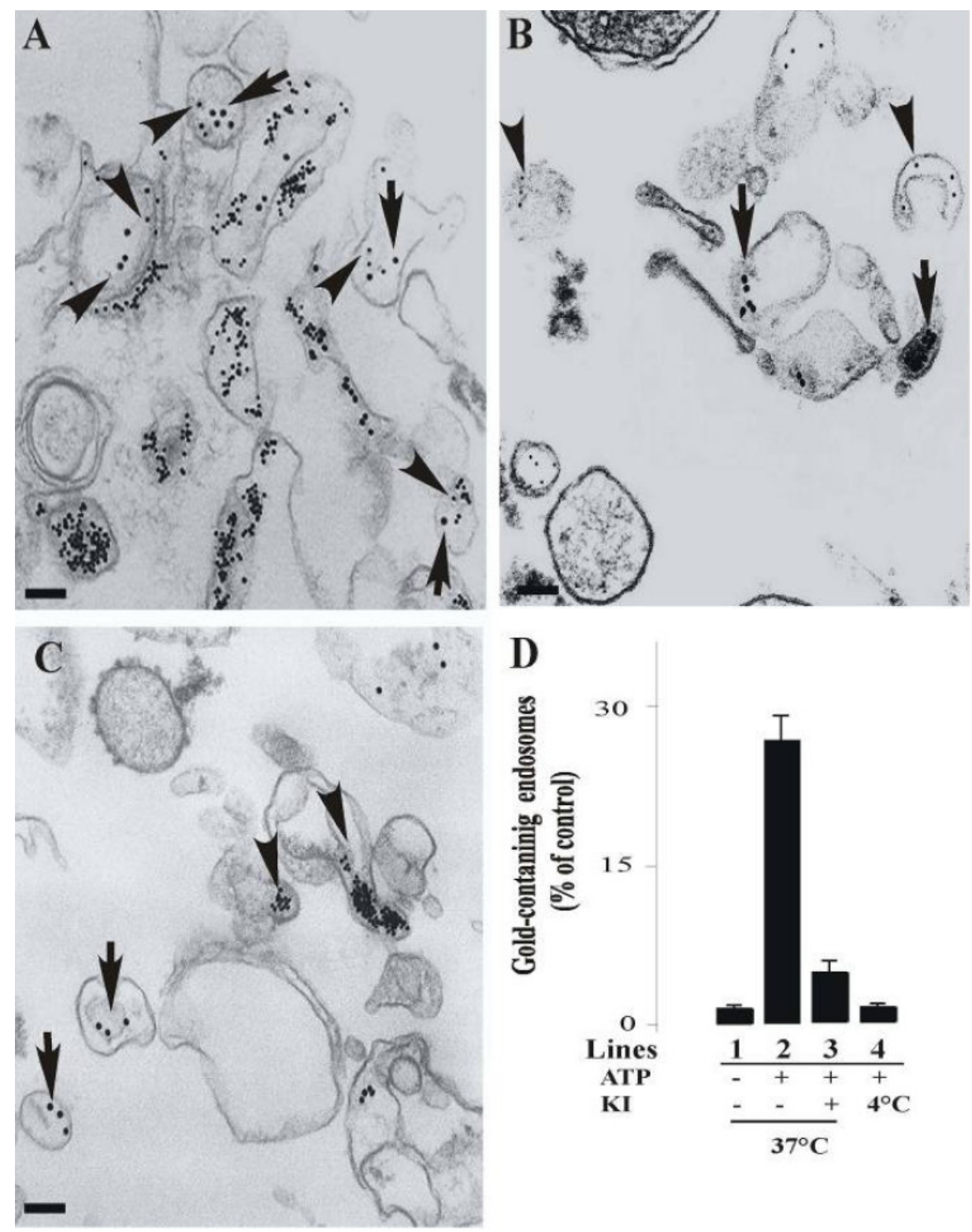

Figure 7. Morphology of in vitro fusion between endosomes. Sub-cellular fractions containing colloidal gold of $10 \mathrm{~nm}$ (B-Ins) and $20 \mathrm{~nm}$ (Av-GAL) loaded in 5-min early endosomes were incubated in vitro for $45 \mathrm{~min}$ in fusion buffer in the presence of $1.2 \mathrm{mg}$ of cytosolic protein $/ \mathrm{ml}$ at $37^{\circ} \mathrm{C}(\mathrm{A})$ and at $4^{\circ} \mathrm{C}(\mathrm{B})$. (C) Fuison was also supplemented by $20 \mu \mathrm{M}$ AG1024 kinase inhibitor in the presence of $1.2 \mathrm{mg}$ of cytosolic protein $/ \mathrm{ml}$ at $37^{\circ} \mathrm{C}$. After the fusion, the samples were fixed in suspension, pelleted, and analyzed for transmission electron microscopy. Bars: $100 \mathrm{~nm}$. (D) For each time of the fusion reaction, the presence of either 10 or $20 \mathrm{~nm}$ gold particles was assessed for at least 2,000 endosomes under four different experimental conditions $\left(-\mathrm{ATP} / 37^{\circ} \mathrm{C}\right.$, lane 1 ; $+\mathrm{ATP} / 37^{\circ} \mathrm{C}$, lane $2 ;+\mathrm{ATP} / 37^{\circ} \mathrm{C}$ in the presence of $20 \mu \mathrm{M}$ AG1024 (KI), lane 3; $+\mathrm{ATP} / 4^{\circ} \mathrm{C}$, lane 4$)$. Results are expressed as a percentage of the total endosomes that contained $10 \mathrm{~nm}$ and $20 \mathrm{~nm}$ gold. Values are means of $+/-\mathrm{SD}, \mathrm{n}=3$. 


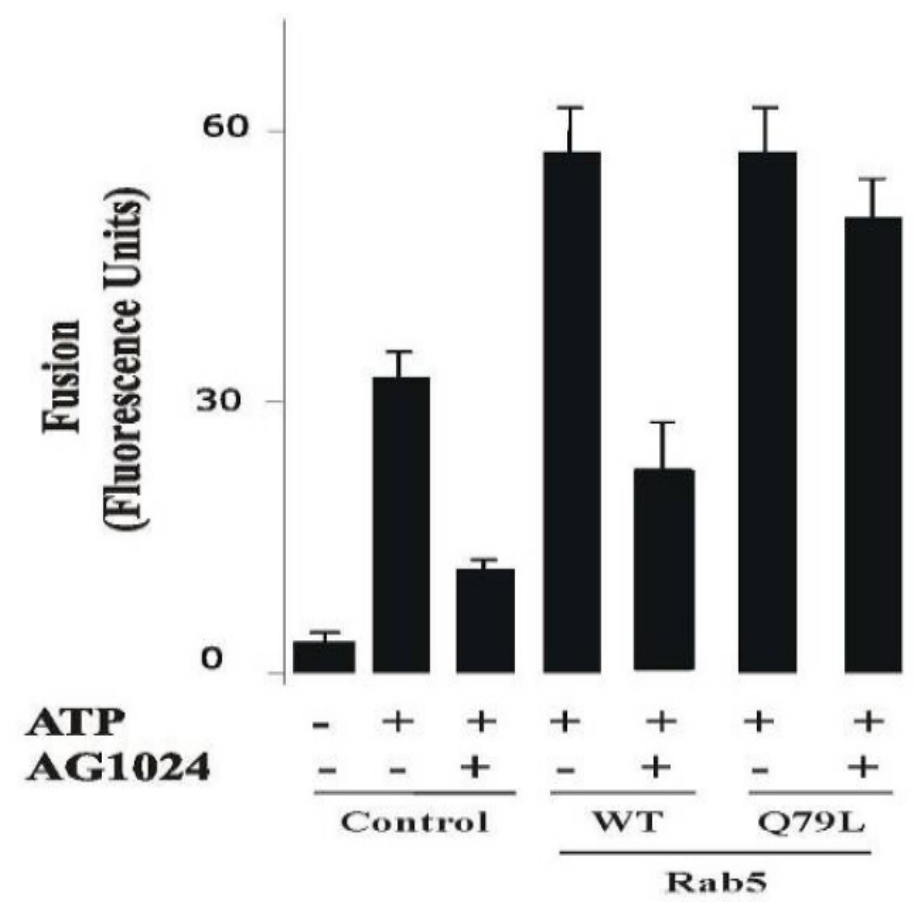

Figure 8. Selective effect of insulin receptor kinase inhibitor on the fusion reaction. The fusion assay was performed under standard conditions as described in Figure 6, either in the absence or in the presence of Rab5:WT or Rab5:Q79L mutant, supplemented with either $20 \mu \mathrm{M}$ AG1024 or ATP in the presence of $0.5 \mathrm{mg} / \mathrm{ml}$ cytosol. The data are presented as means $\pm \mathrm{SD}$ of four independent experiments. 


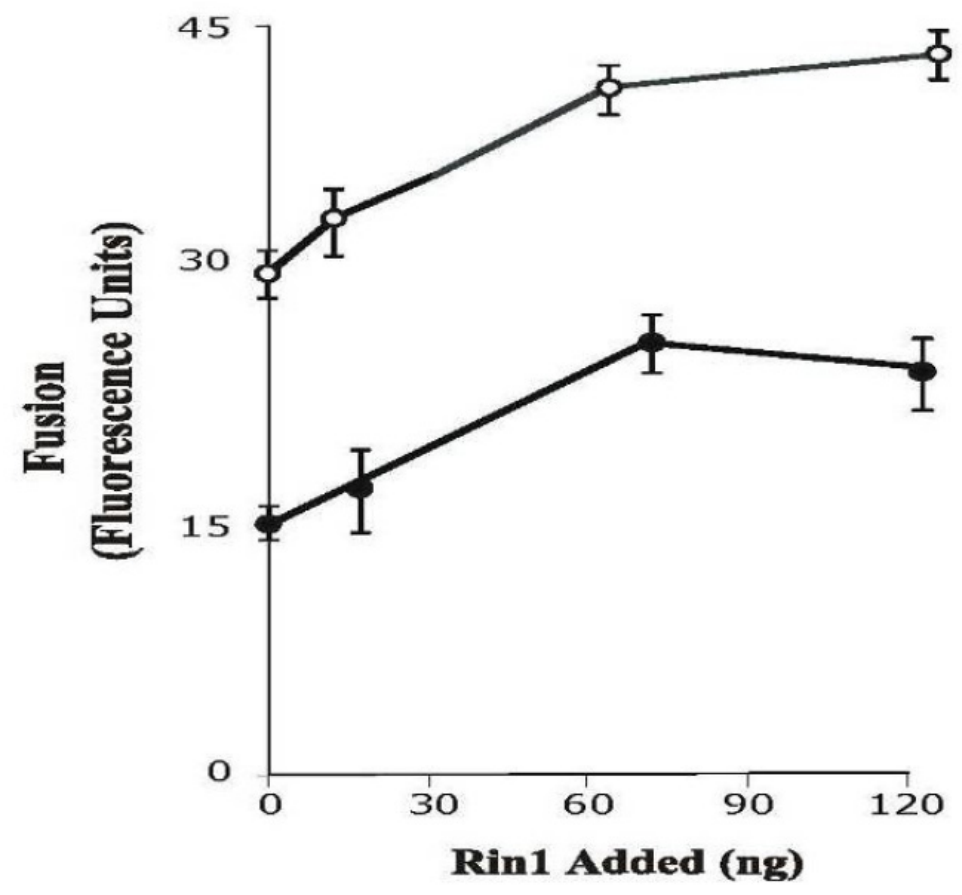

Figure 9. Insulin receptor kinase inhibitor blocks endosome fusion stimulated by Rin1. The fusion assay was performed under standard conditions as described in Figure 6 , either in the absence or in the presence of different concentrations of Rin1 supplemented with several concentrations of AG1084 ro addition, •: $20 \mu \mathrm{M}$ ) inhibitor in the presence of $0.5 \mathrm{mg} / \mathrm{ml}$ cytosol. The data are presented as means $\pm \mathrm{SD}$ of four independent experiments. 

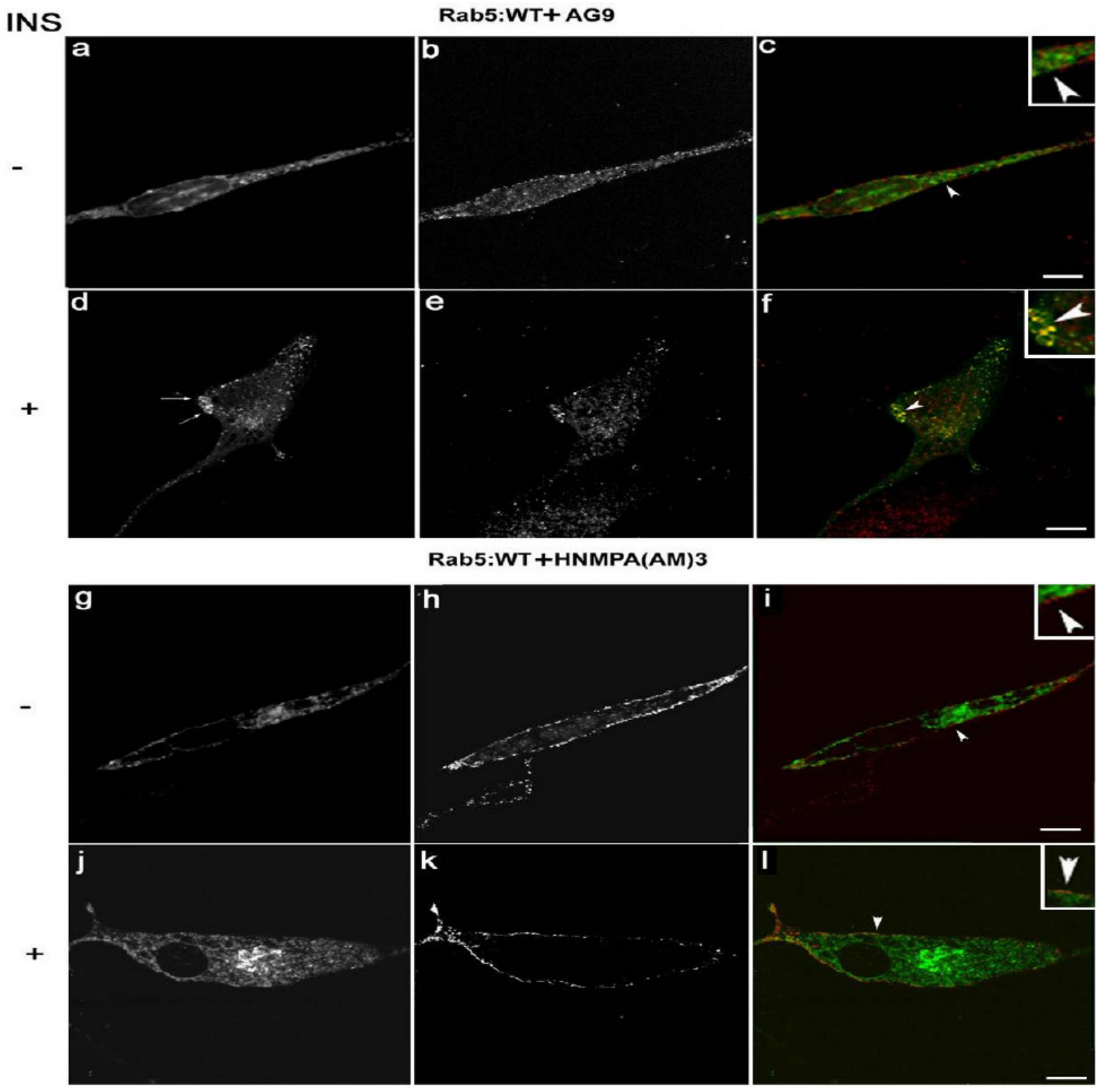

Rab5:WT +HNMPA(AM)3
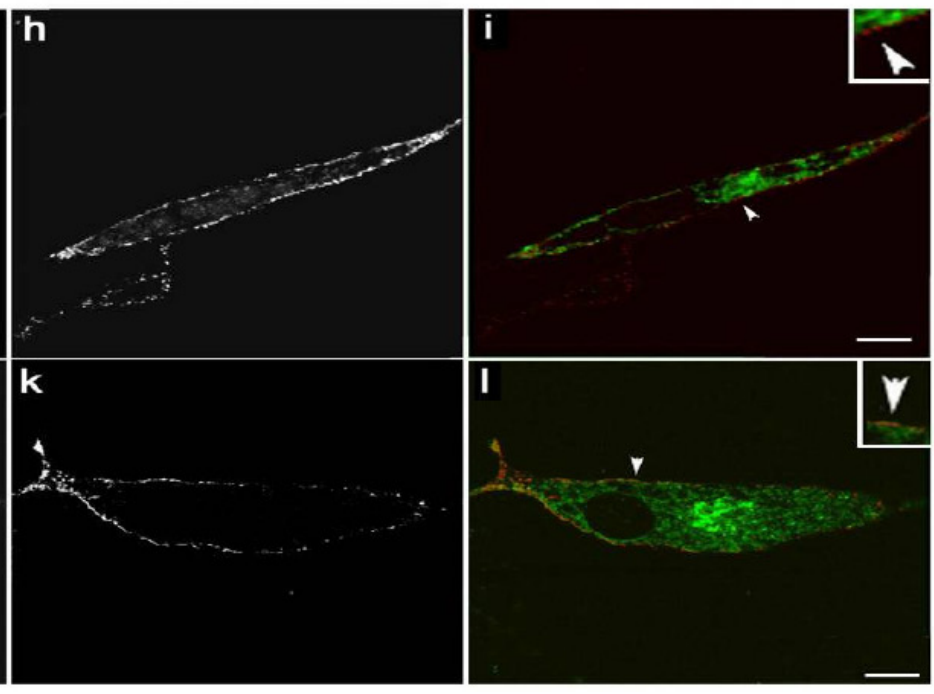

Figure 10. Confocal immunofluorescence analysis of cells co-expressing Insulin receptor, Rab5: wild-type in the presence of insulin receptor tyrosine kinase inhibitor. NIH-IR cells were transfected with plasmids encoding GFP-Rab5: wild type (A-L) in the absence (A-C; G-I) or in the presence of $100 \mathrm{ng} / \mathrm{ml}$ insulin (D-F; J-L). Cells were also supplemented with $100 \mu \mathrm{M}$ HNMPA-(AM) $)_{3}$ inhibitors (G-L) and inactive analog (AG9) (A-F). $100 \mathrm{ng} / \mathrm{ml}$ insulin was bound to the cells at $4^{\circ} \mathrm{C}$ for $60 \mathrm{~min}$. The cells were then washed with ice-cold PBS and then incubated at $37^{\circ} \mathrm{C}$ for $8 \mathrm{~min}$. Subsequently, the cells were washed three times with ice-cold PBS and fixed with 4\% paraformaldehyde and then were permeabilized with $0.1 \%$ Triton X-I00 prior to incubation with antibodies. The primary antibodies used were rabbit anti-insulin receptor. The secondary antibodies used were Alexa564-labelled donkey anti-rabbit IgG antibodies. Yellow color indicates areas of co-localization between the internalized 
insulin receptor and the overexpressed GFP-Rab5 proteins (C, F, I and L). An inactive analog (AG9) was used as control. The optical sections viewed are $0.4 \mu \mathrm{m}$. Size bars, 10 $\mu \mathrm{m}$. 


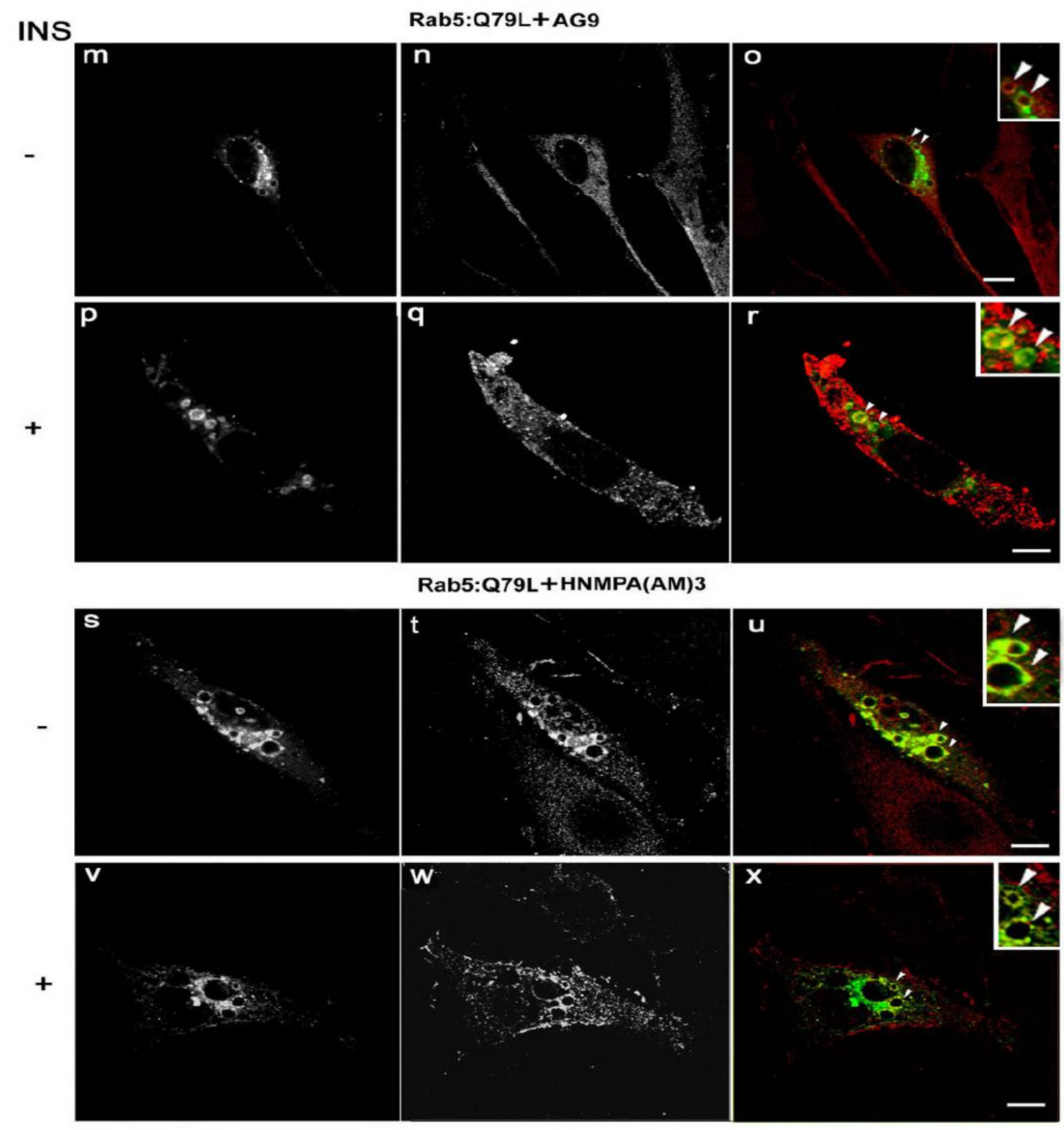

Figure 11. Confocal immunofluorescence analysis of cells co-expressing Insulin receptor, Rab5: Q79L mutant in the presence of insulin receptor tyrosine kinase inhibitor. NIH-IR cells were transfected with plasmids encoding GFP-Rab5: Q79L (A$\mathrm{L}$ ) and in the absence (A-C and G-I) or in the presence of insulin (D-F and J-L). Cells were also supplemented with $100 \mu \mathrm{M}$ HNMPA-(AM) $)_{3}$ inhibitors (G-L) and inactive analog (AG9) (A-F). $100 \mathrm{ng} / \mathrm{ml}$ insulin was bound to cells at $4^{\circ} \mathrm{C}$ for $60 \mathrm{~min}$. Cells were washed with ice-cold PBS and then incubated at $37^{\circ} \mathrm{C}$ for $8 \mathrm{~min}$. Subsequently, the cells were washed three times with ice-cold PBS and fixed with $4 \%$ paraformaldehyde and then permeabilized with $0.1 \%$ Triton X-I00 prior to incubation with antibodies. The primary antibodies used were rabbit anti-insulin receptor. The secondary antibodies used 
were Alexa564-labelled donkey anti-rabbit IgG antibodies. Yellow color indicates areas of colocalization between the internalized insulin receptor and the overexpressed GFPRab5 proteins (C, F, I and L). An inactive analog (AG9) was used as control. The optical sections viewed are $0.4 \mu \mathrm{m}$. Size bars, $10 \mu \mathrm{m}$. 

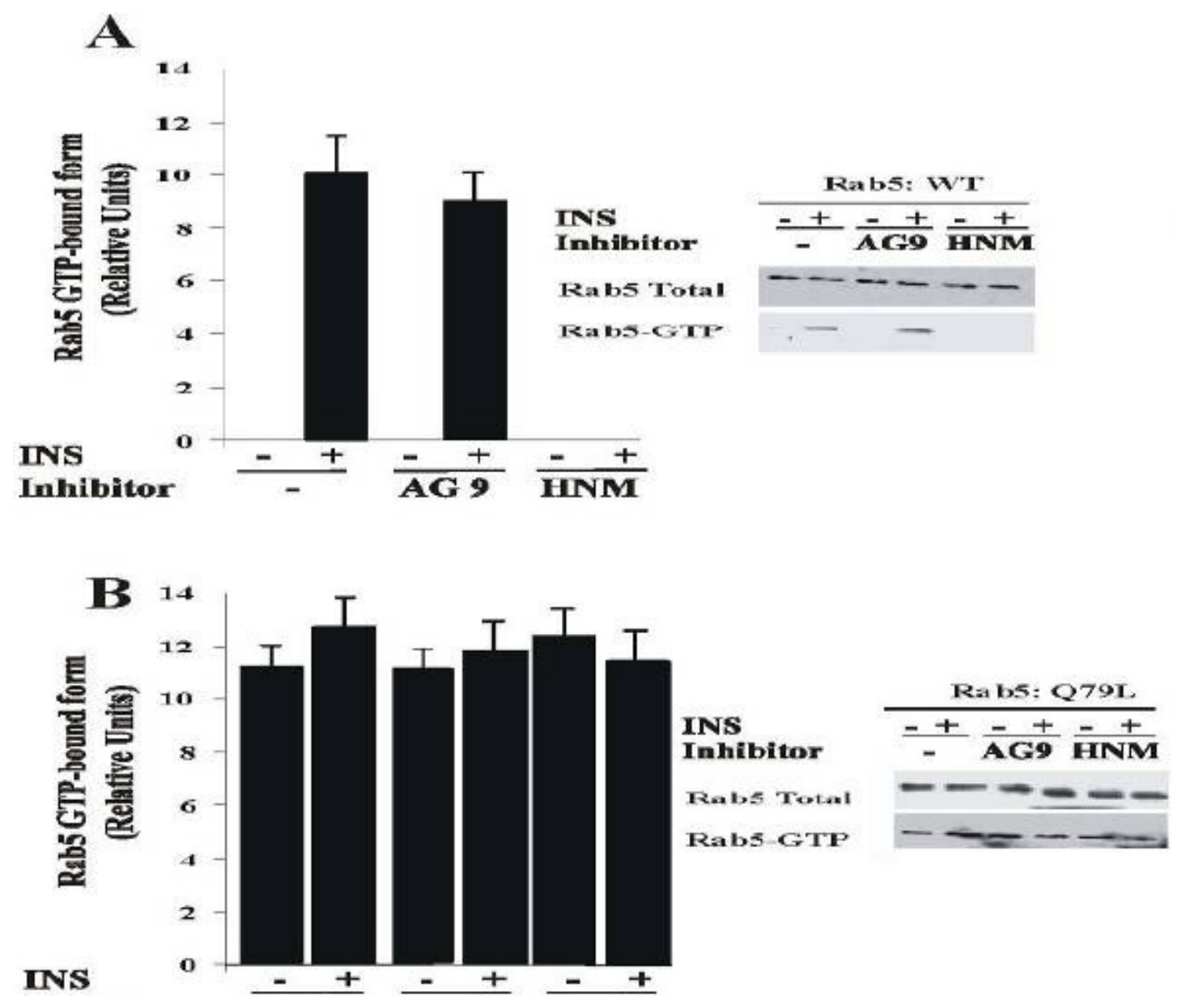

Figure 12. Insulin receptor tyrosine kinase inhibitor blocks the activation of Rab5 in intact cells. NIH-IR were transfected with plasmids encoding GFPRab5: wild type (A) and GFP-Rab5: Q79L mutant (B) in the absence or in the presence of $100 \mathrm{ng} / \mathrm{ml}$ insulin containing either $100 \mu \mathrm{M}$ AG9 or $100 \mu \mathrm{M}$ HNMPA-(AM) $)_{3}$ (HNM) inhibitors as indicated in the Figure. Insulin was bound to the cells at $4^{\circ} \mathrm{C}$ for $60 \mathrm{~min}$. The cells were washed and then incubated at $37^{\circ} \mathrm{C}$ for $8 \mathrm{~min}$. Subsequently, the cells were washed three times with ice-cold PBS, lysed and incubated with gluthathione beads either in the presence of GST alone or GST-EEA 1 at $4^{\circ} \mathrm{C}$ for $60 \mathrm{~min}$. After incubation, the beads were washed and the presence of activated Rab5 was analyzed by Western blotting using antiRab5 antibodies. The data are presented as means \pm SD of four independent experiments. 


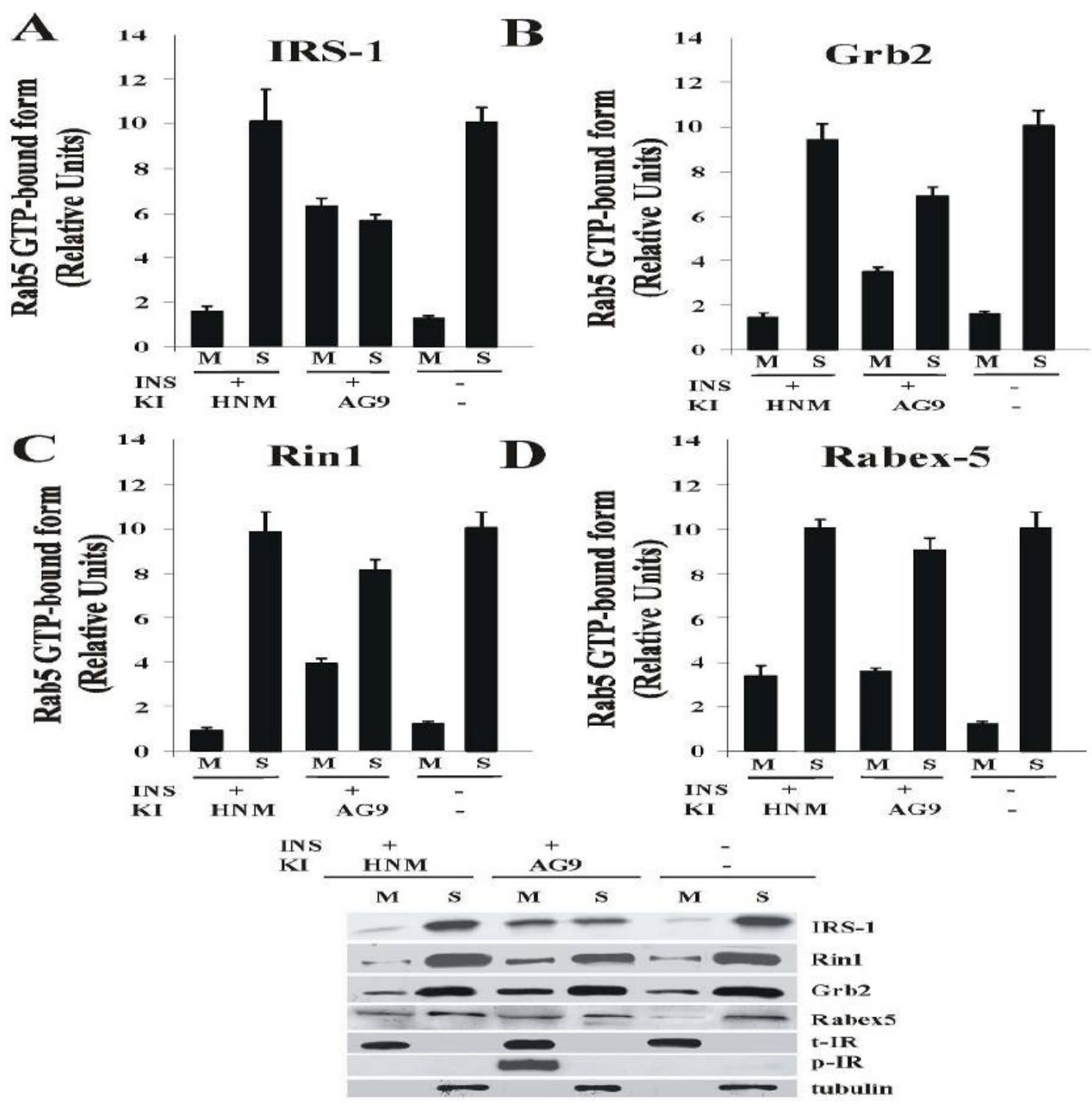

Figure 13. Effect of HNMPA-(AM) 3 inhibitor on the tyrosine phosphorylation of Insulin receptor and recruitment of Rin1. Cells were incubated in the absence or in the presence of $100 \mathrm{ng} / \mathrm{ml}$ insulin either $100 \mu \mathrm{M}$ AG9 or $100 \mu \mathrm{M}$ HNMPA-(AM) $)_{3}$ inhibitor as described in the Figure. After treatment, cells were washed with ice cold PBS and incubated at $37^{\circ} \mathrm{C}$ for 8 minutes. Cells were then washed again using ice-cold PBS, homogenized and membrane fractions were prepared as described in Materials and Methods. Membrane (M) and cytosol (S) fractions were treated with sample buffer and proteins were subject to SDS-PAGE, blotted to a nitrocellulose membrane, and antibodies specific to IRS-1 (A), Grb2 (B), Rin1 (B), Rabex-5 (C), tubulin, phospoinsulin receptor $(\mathrm{p})$-IR and total (t)-IR, and tubulin were used to visualize these proteins by Western blot analysis. A representative experiment is shown (E). This experiment was repeated at least three times, and the results were reproducible. The data are presented as means \pm SD of three independent experiments. 


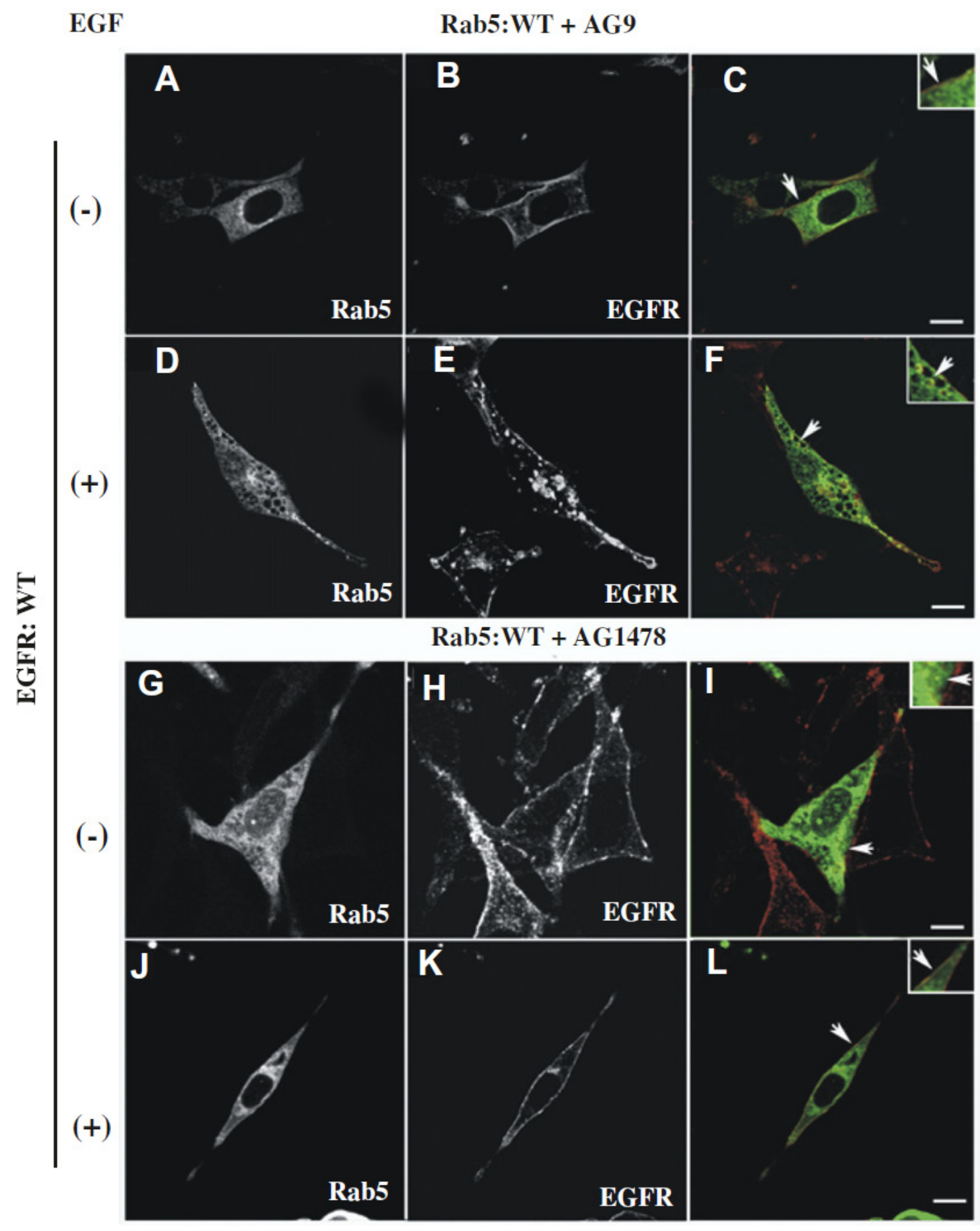




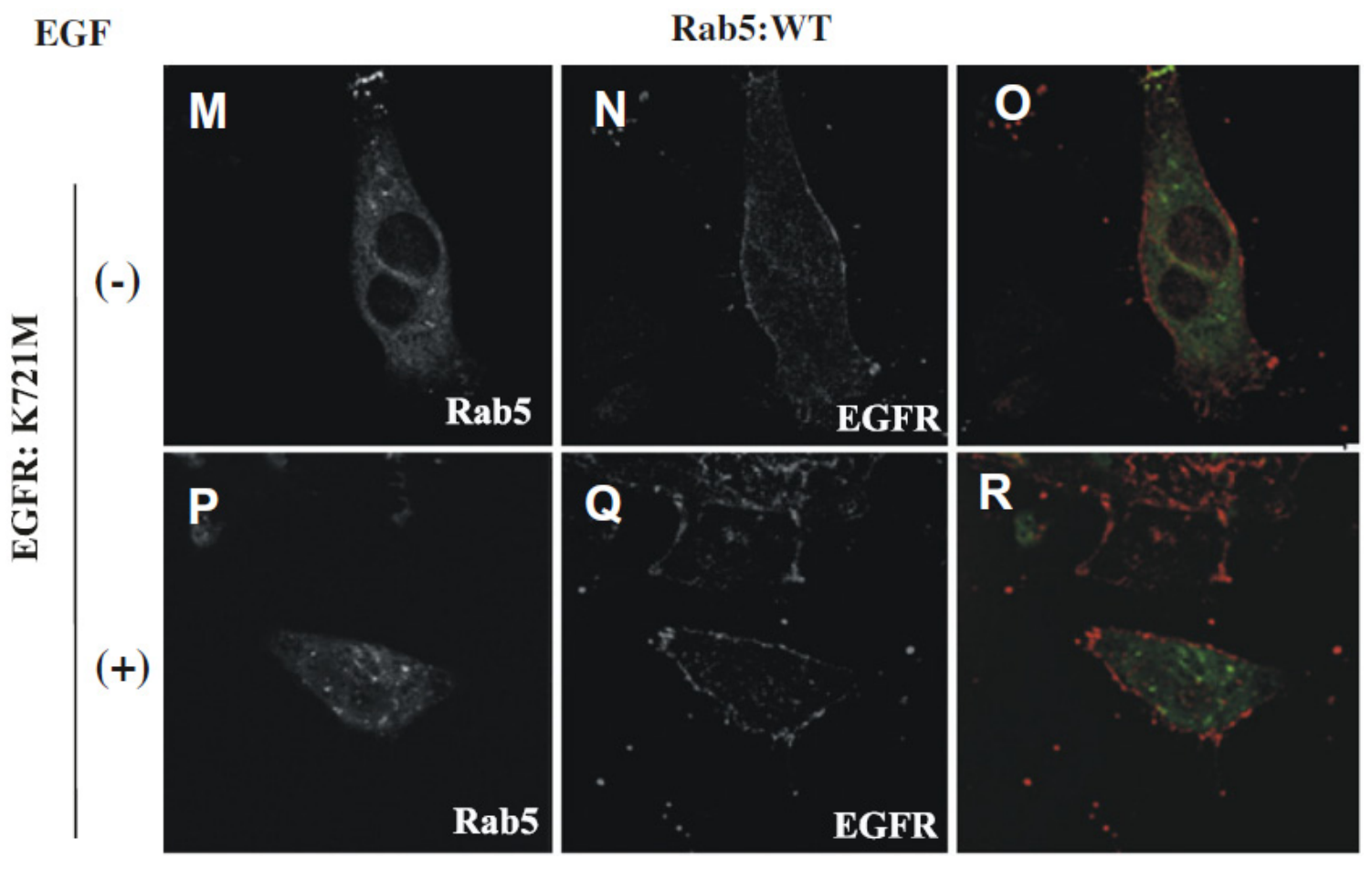

Figure 14. Confocal immunofluorescence analysis of cells co-expressing EGFreceptor and Rab5: wild-type in the presence of EGF-receptor tyrosine kinase inhibitor. NR6 cells expressing either EGF-receptor wild type (A-L) or EGF-receptor K721M mutant (M-R) were transfected with plasmids encoding GFP-Rab5: wild type (A$\mathrm{R}$ ) in the absence (A-C, G-I, M-O) or in the presence of EGF (D-F, J-L, P-R). Cells were also supplemented with $100 \mathrm{nM}$ AG9 inactive analog (A-F) or $100 \mathrm{nM}$ AG1478 inhibitors (G-L). $100 \mathrm{ng} / \mathrm{ml}$ EGF was bound to the cells at $4^{\circ} \mathrm{C}$ for $60 \mathrm{~min}$. Cells were then washed with ice-cold PBS, incubated at $37^{\circ} \mathrm{C}$ for $8 \mathrm{~min}$, washed three times with ice-cold PBS, fixed with $4 \%(\mathrm{w} / \mathrm{v})$ paraformaldehyde and then permeabilized with $0.1 \%$ (v/v) Triton X-100 prior to incubation with antibodies. The primary antibody used was polyclonal rabbit anti-EGF-receptor. The secondary antibody used was Alexa564labelled donkey anti-rabbit IgG. Yellow color indicates areas of co-localization between the internalized EGF-receptor (B, E, H, K, N and Q) and the overexpressed GFP-Rab5 proteins (A, D, G, J, M and P). An inactive analog (AG9) was used as control. The optical sections viewed are $0.4 \mu \mathrm{m}$. Size bars, $10 \mu \mathrm{m}$. 


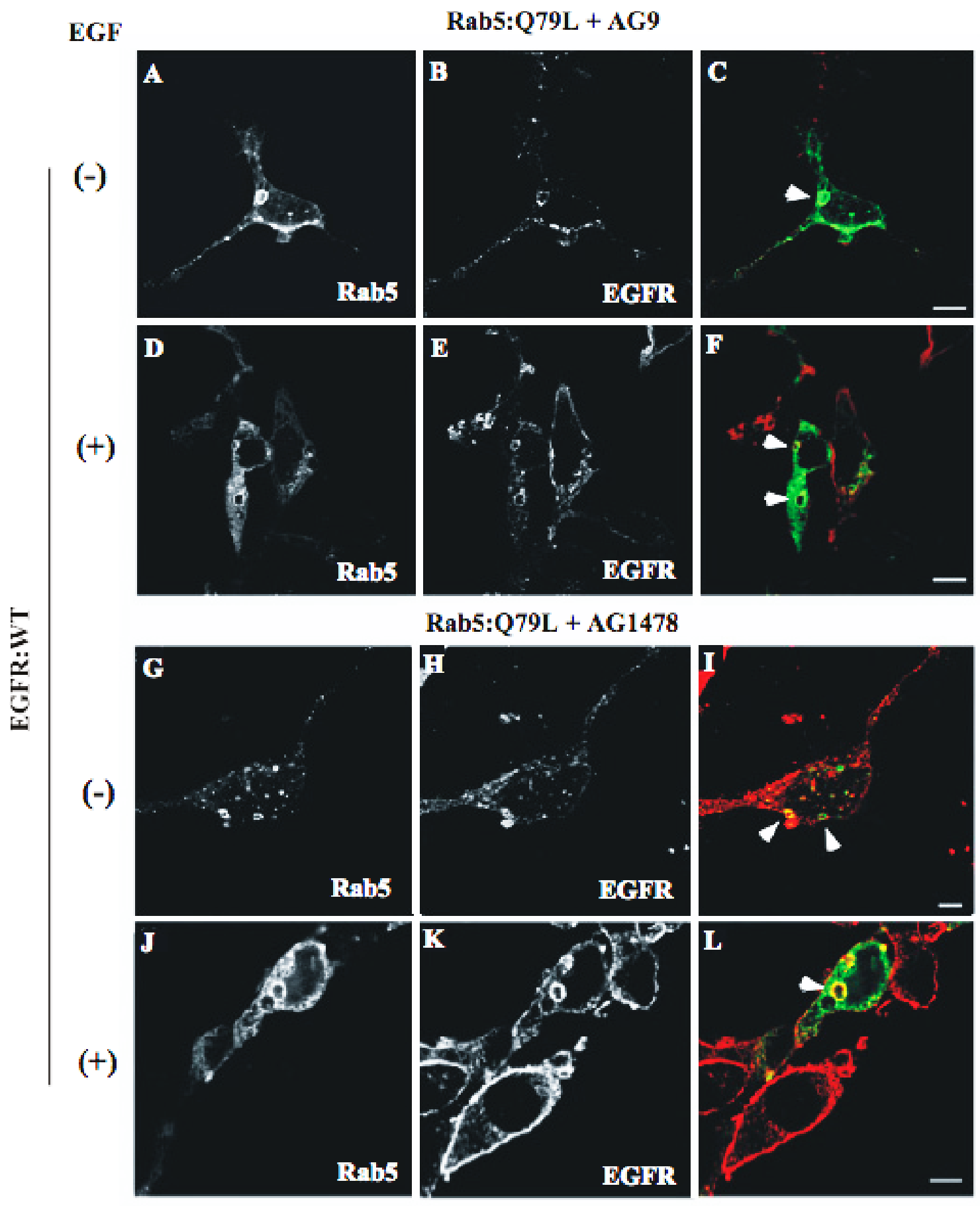




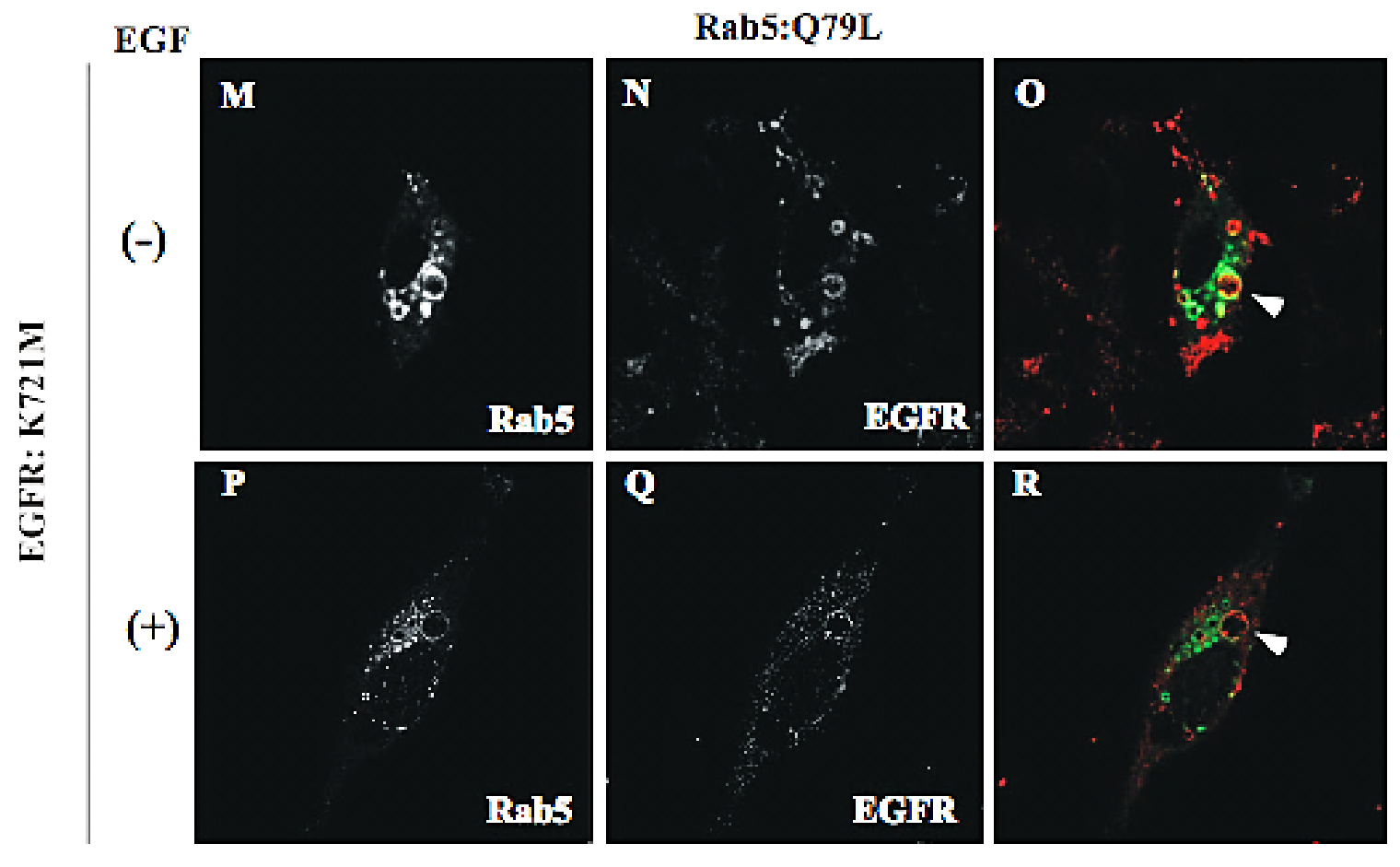

Figure 15. Confocal immunofluorescence analysis of cells co-expressing EGFreceptor, Rab5: Q79L mutant in the presence of EGF-receptor tyrosine kinase inhibitor. NR6 cells expressing human EGF-receptor wild type (A-L) and catalytically inactive mutant (K721M) (M-R) were transfected with plasmids encoding GFP-Rab5: Q79L (A-R) and then incubated in the absence (A-C, G-I and M-O) or in the presence of EGF (D-F, J-L and P-R). Cells were also supplemented with $100 \mathrm{nM}$ AG1478 inhibitor (G-L) and $100 \mathrm{nM}$ AG9 (inactive analog) (A-F). $100 \mathrm{ng} / \mathrm{ml}$ EGF was bound to the cells at $4^{\circ} \mathrm{C}$ for $60 \mathrm{~min}$. Cells were then washed with ice-cold PBS, incubated at $37^{\circ} \mathrm{C}$ for $8 \mathrm{~min}$, washed three times with ice-cold PBS, fixed with $4 \%$ paraformaldehyde and then were permeabilized with $0.1 \%$ Triton X-100 prior to incubation with rabbit anti-EGF-receptor and Alexa564-labelled donkey anti-rabbit antibodies, respectively. Yellow color indicates areas of co-localization between the internalized EGF-receptor and the overexpressed GFP-Rab5: Q79L mutant (C, F, I, L, O and R). An inactive analog (AG9) was used as control. The optical sections viewed are $0.4 \mu \mathrm{m}$. Size bars, $10 \mu \mathrm{m}$. 
$\mathbf{A}$

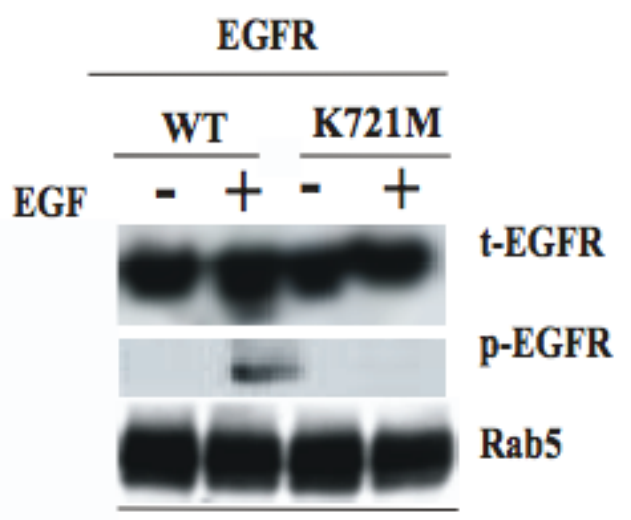

Rab5: WT

B

EGFR

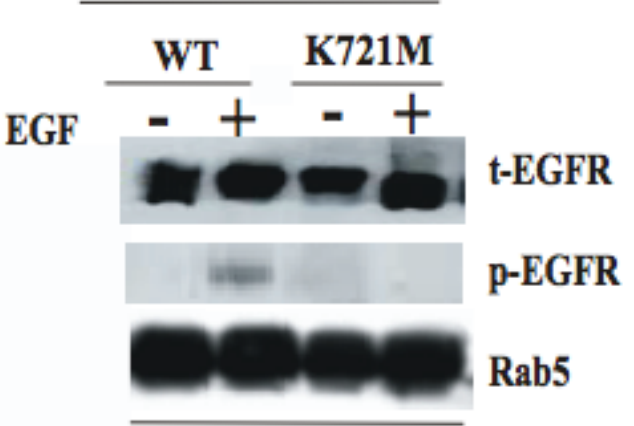

Rab5: Q79L

Figure 16. Expression and tyrosine phosphorylation of EGF-receptor in cells expressing Rab5 proteins. NR6 cells expressing human EGF-receptor wild type (WT) and catalytically inactive mutant $(\mathrm{K} 721 \mathrm{M})$ were transfected with plasmids encoding GFP-Rab5: wild type (A) and GFP-Rab5:Q79L mutant (B) as indicate in the Figure. Cells were starved, stimulated or not for $8 \mathrm{~min}$ with EGF $(100 \mathrm{ng} / \mathrm{ml})$, and lysed in iceold lysis buffer as described in Material and Methods. Cell lysates were then subjected to SDS-PAGE and analyzed by immunoblotting using anti-phospho (p) and anti-total (t) EGF-receptor and anti-Rab5 antibodies. The experiment was repeated twice with similar results. 
A

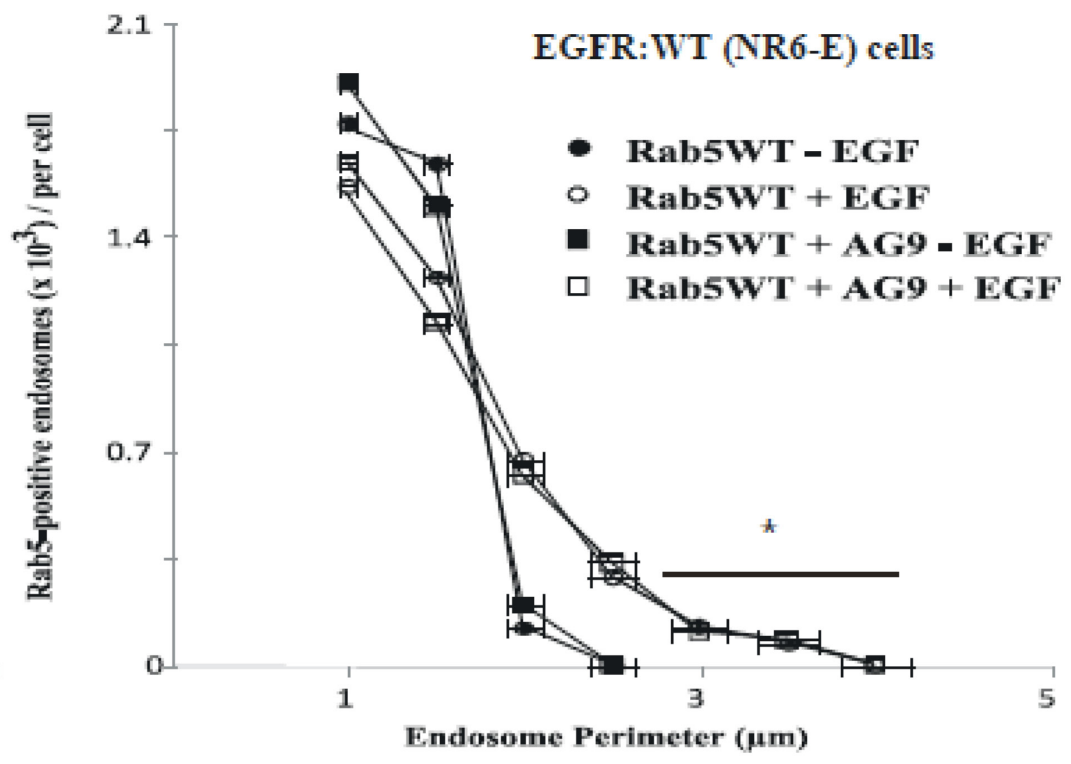

B

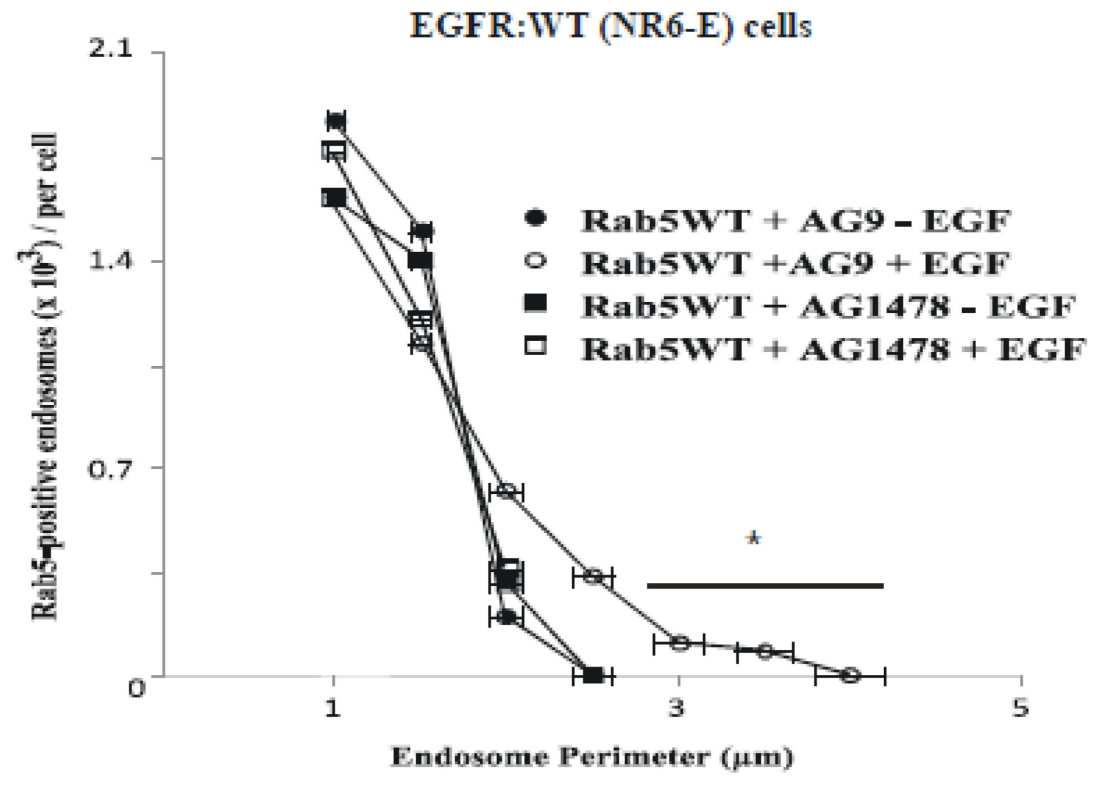



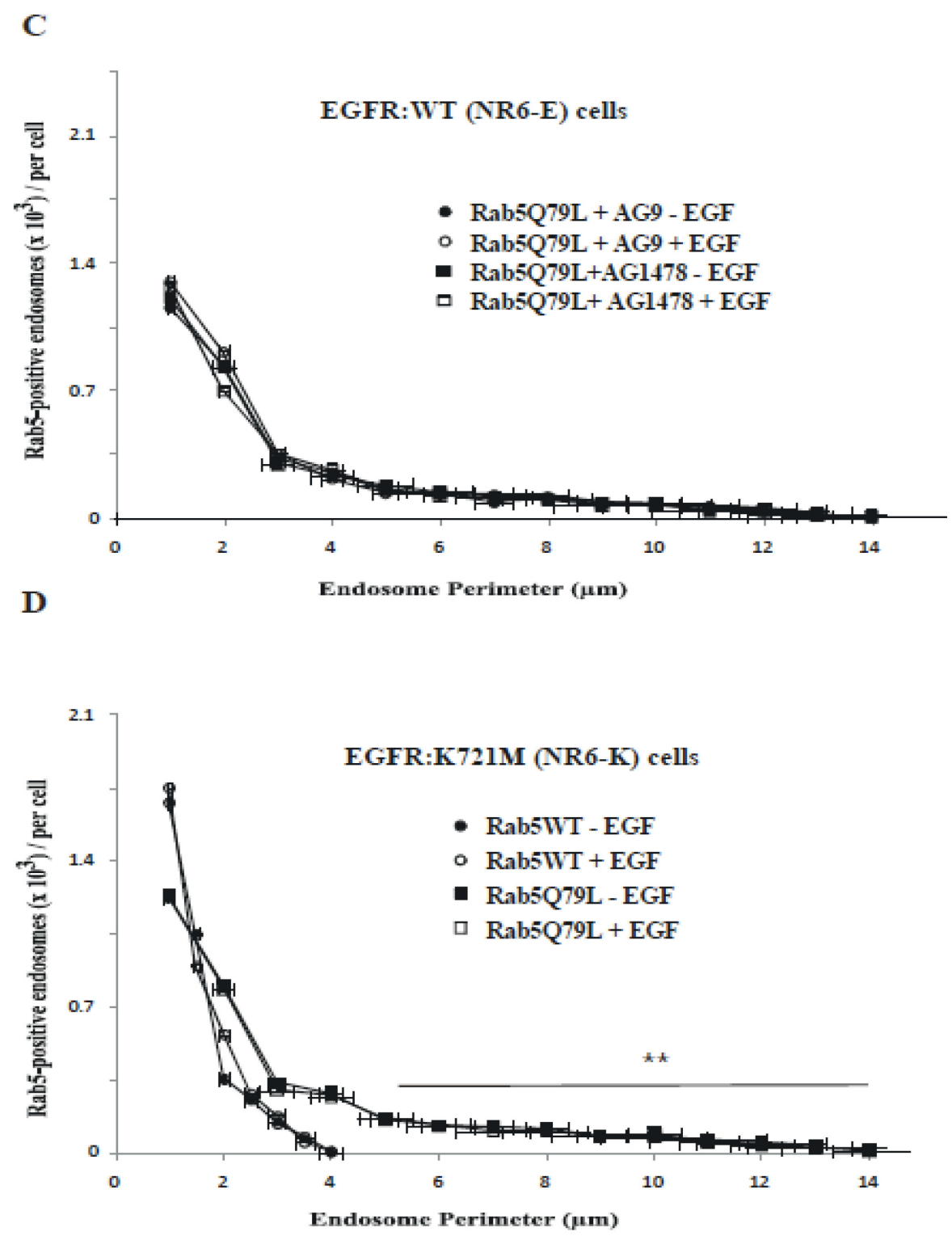

Figure 17. Effect of AG1478 on the perimeter of Rab5-positive endosomes. Perimeters of Rab5-positive endosomes from at least 200 cells were measured using the NIH Image J software from immunofluorescence images acquired using a Leica TCS SP2 confocal microscope in NR6 cells for each experimental condition. (A-B) Histograms of Rab5-positive endosomes/cell vs. the endosome size were determined in NR6-E cells expressing Rab5 wild type (WT) alone or containing either $100 \mathrm{nM}$ AG9 or $100 \mathrm{nM}$ AG1478 in the absence or in the presence of $100 \mathrm{ng} / \mathrm{ml} \mathrm{EGF.} \mathrm{Asterisk} \mathrm{(*)} \mathrm{denotes} \mathrm{an}$ extended tail of Rab5:WT endosomes distribution in the presence of EGF. (C) Histograms of Rab5-positive endosomes vs. the endosome size were determined in NR6E cells expressing Rab5: Q79L containing either 100 nM AG9 or 100 nM AG1478 in the absence or in the presence of $100 \mathrm{ng} / \mathrm{ml}$ EGF. (D) Histograms of Rab5-positive endosomes vs. the endosome size were determined in NR6-K cells expressing Rab5: 
Q79L in the absence or in the presence of $100 \mathrm{ng} / \mathrm{ml}$ EGF. Asterisks (**) denote an extended tail of Rab5: Q79L endosomes distribution in the presence of EGF. The data are presented as means \pm SD of four independent experiments. 
A
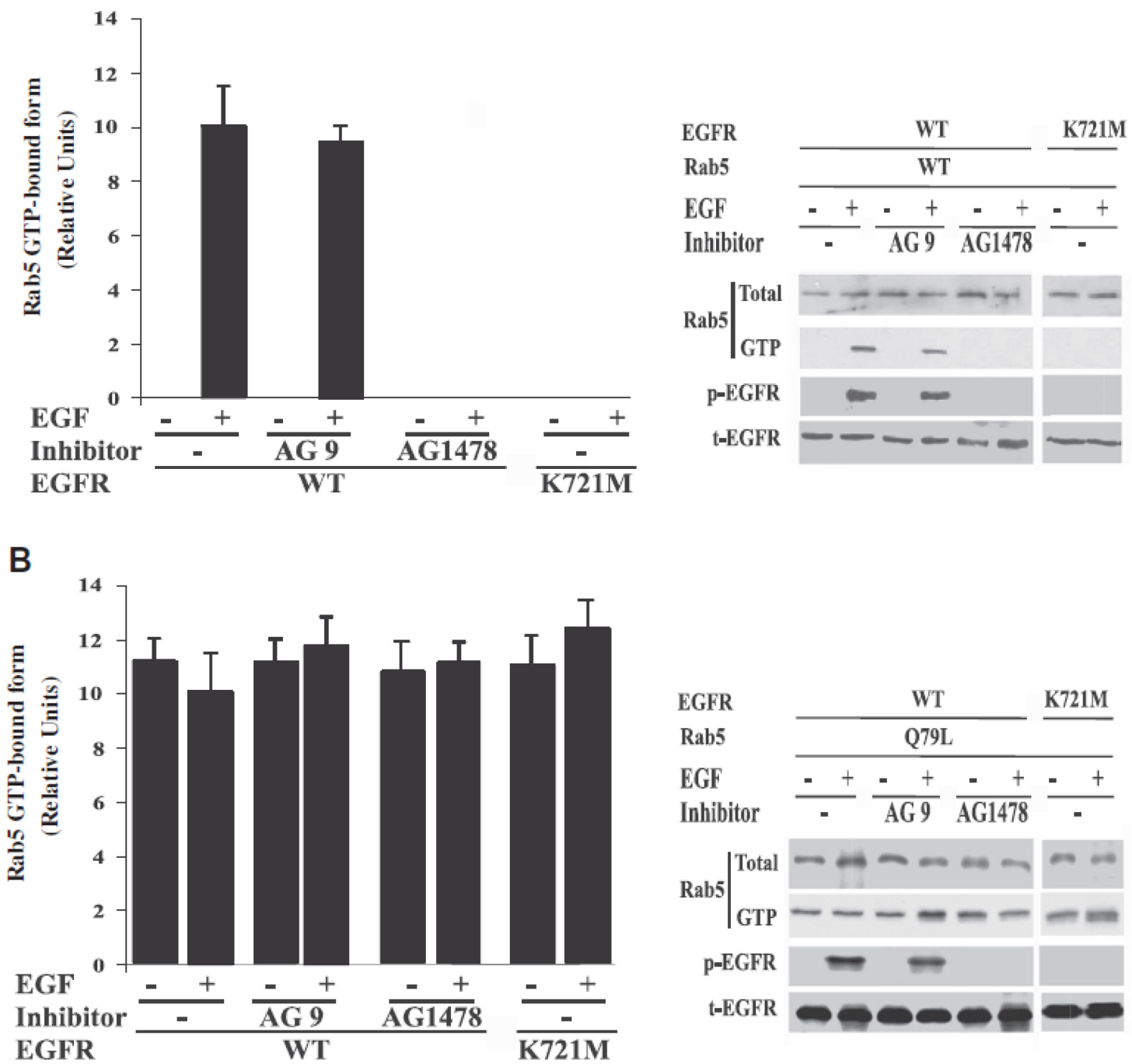

Figure 18. AG1478 inhibitor blocks the activation of Rab5 in intact cells. NR6 cells expressing either EGF-receptor wild type or EGF-receptor K721M mutant were transfected with plasmids encoding GFP-Rab5: wild type (A) and GFP-Rab5: Q79L mutant (B) in the absence or in the presence of EGF and $100 \mathrm{nM}$ AG1478 inhibitor as indicated in the Figure. $100 \mathrm{ng} / \mathrm{ml} \mathrm{EGF}$ was bound to the cells at $4^{\circ} \mathrm{C}$ for $60 \mathrm{~min}$. The cells were washed and then incubated at $37^{\circ} \mathrm{C}$ for $8 \mathrm{~min}$. Subsequently, the cells were washed three times with ice-cold PBS, lysed and incubated with glutathione beads either in the presence of GST alone or GST-EEA1 at $4{ }^{\circ} \mathrm{C}$ for $60 \mathrm{~min}$. After incubation, the beads were washed and the presence of activated Rab5 was analyzed by Western blotting. The data are presented as means $\pm \mathrm{SD}$ of four independent experiments. 
A
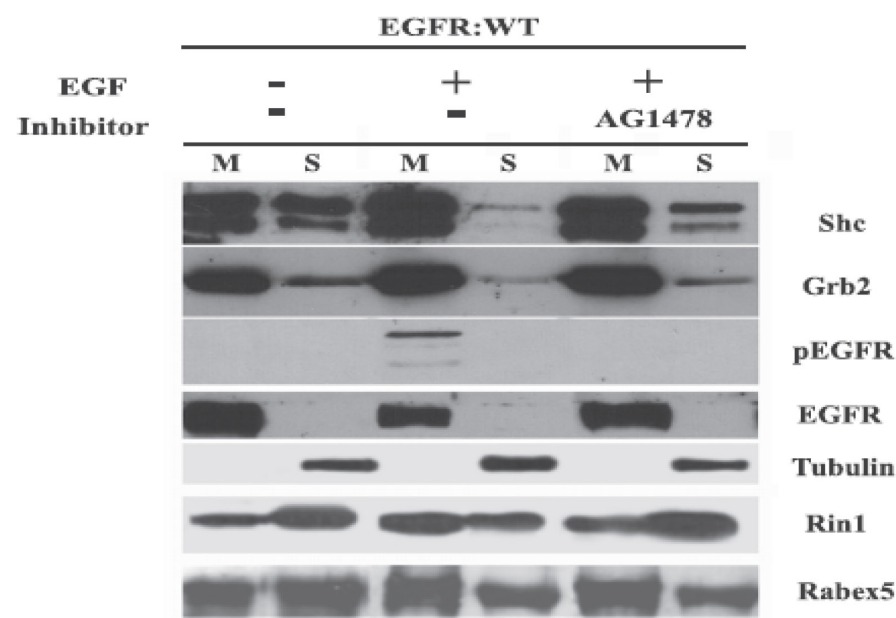

B

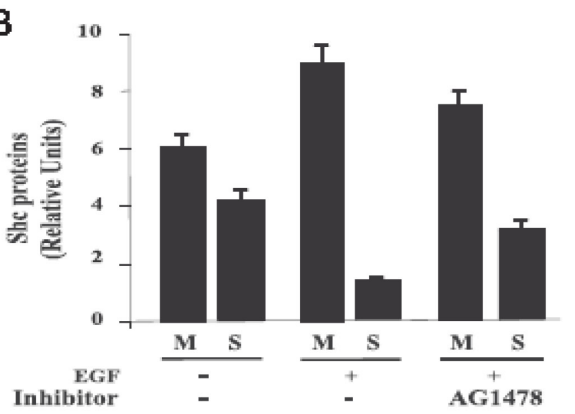

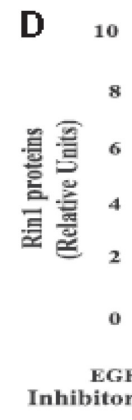

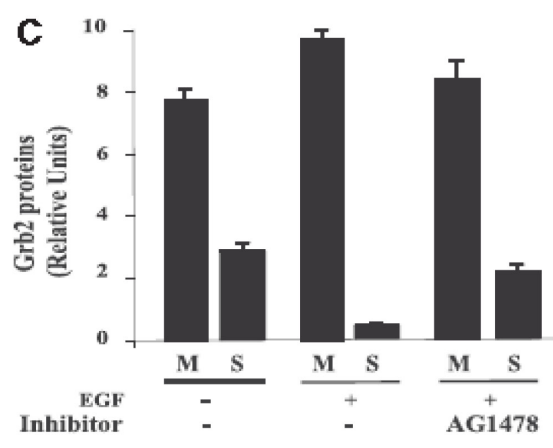

E

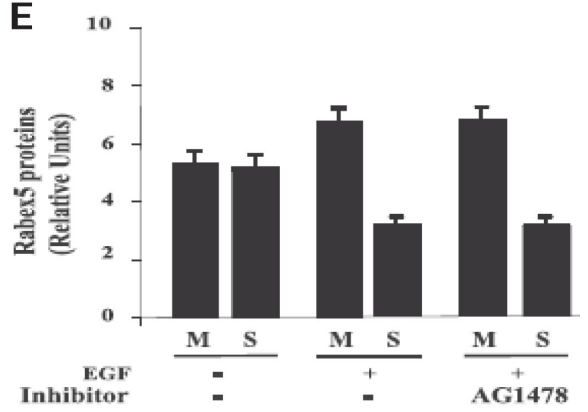

Figure 19. Effect of AG1478 inhibitor on the tyrosine phosphorylation of EGFreceptor and recruitment of Rin1 in intact cells. NR6 cells expressing EGF-receptor

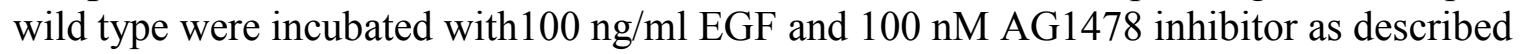
in Materials and Methods. After treatment, cells were washed with ice cold PBS and incubated at $37^{\circ} \mathrm{C}$ for 8 minutes. Cells were then washed again using ice-cold PBS, homogenized and membrane fractions were prepared as described in Materials and Methods. Membrane [M] and cytosol [S] fractions (A) were treated with sample buffer and proteins were subject to SDS-PAGE, blotted to a nitrocellulose membrane, and antibodies specific to Shc (B), Grb2 (C), Rin1 (D), Rabex-5 (E), phospho(p)-EGFreceptor, total $(\mathrm{t})$-EGF-receptor, and tubulin (A) were used to visualize these proteins by Western blot analysis. Relative levels of these proteins were determined by densitometry. Data represents the mean $\pm \mathrm{SD}$ of four independent experiments. 


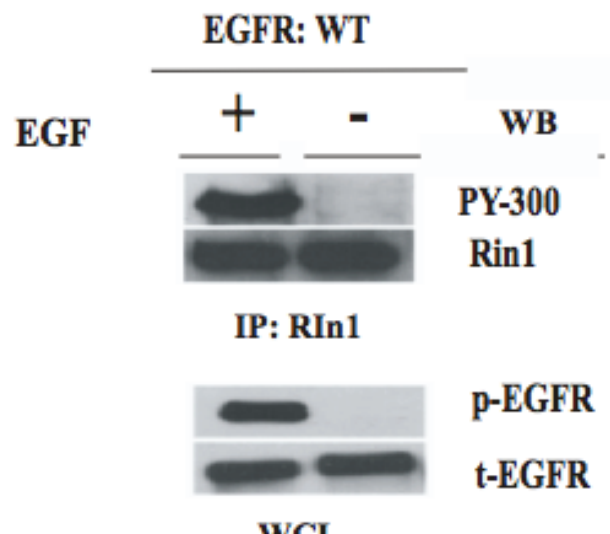

Figure 20. Rin1 is not tyrosine phosphorylated by EGF. NR6 cells expressing human EGF-receptor wild type (WT) were transfected with plasmids encoding Rin1: wild type as indicated in the figure. Cells were serum-starved, stimulated or not for 8 min with EGF $(100 \mathrm{ng} / \mathrm{ml})$, lysed and then immunoprecipitated with anti-Rin 1 antibodies as indicated in Material and Methods. The immunoprecipitates were then subjected to SDS-PAGE and analyzed by immunoblotting using anti-Rin1 and anti-phosphotyrosine (PY-300) antibodies, respectively. Whole cell lysates (WCL) were analyzed utilizing antibodies recognizing both total $(\mathrm{t})$ and phospho $(\mathrm{p})$-EGF receptor. The experiment was repeated twice with similar results. 
$\mathbf{A}$

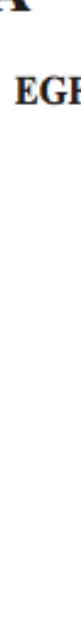

EGFR: K721M
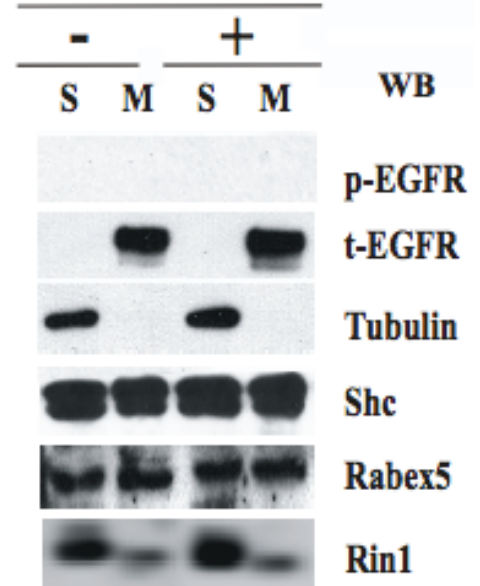

$\mathbf{C}$

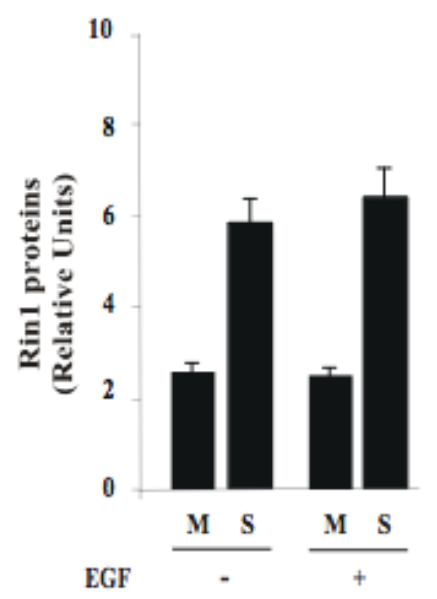

B

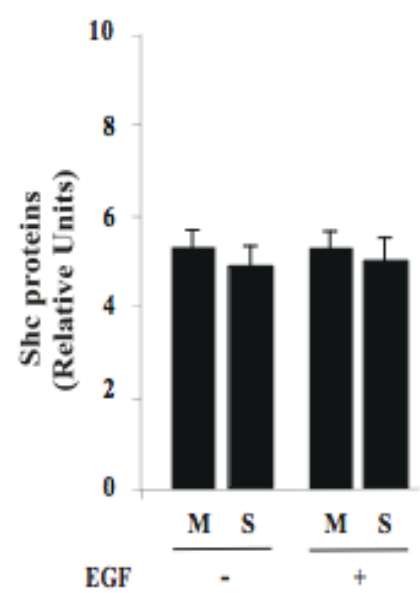

D

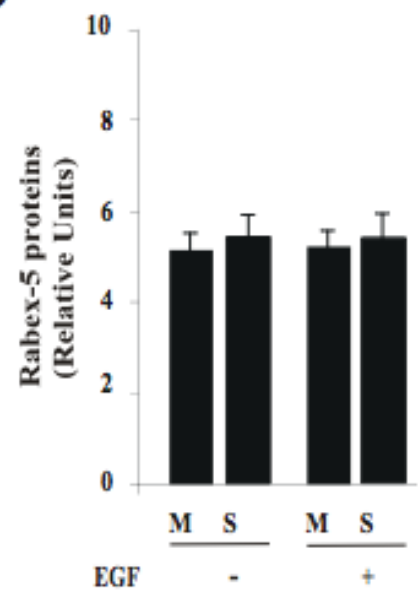

Figure 21. Expression of EGF-receptor catalytically inactive mutant affects the recruitment of Rin1 to membrane upon stimulation of EGF. NR6 cells expressing EGF-receptor catalytically inactive mutant (K721M) were incubated in the absence or in the presence of $100 \mathrm{ng} / \mathrm{ml} \mathrm{EGF}$ at $37^{\circ} \mathrm{C}$ for 8 minutes as indicated in Materials and Methods. Cells were then washed again using ice-cold PBS, homogenized and membrane fractions were prepared as described in Materials and Methods. Membrane [M] and cytosol $[\mathrm{S}]$ fractions were (A) treated with sample buffer and proteins were subject to SDS-PAGE, blotted to a nitrocellulose membrane, and antibodies specific to Shc (B), Rin1 (C), Rabex-5 (D), phospho(p)-EGF-receptor and total(t)-EGF-receptor, and tubulin (A) were used to visualize these proteins by Western blot analysis. Relative levels of these proteins were determined by densitometry. Data represents the mean $\pm \mathrm{SD}$ of three independent experiments. 

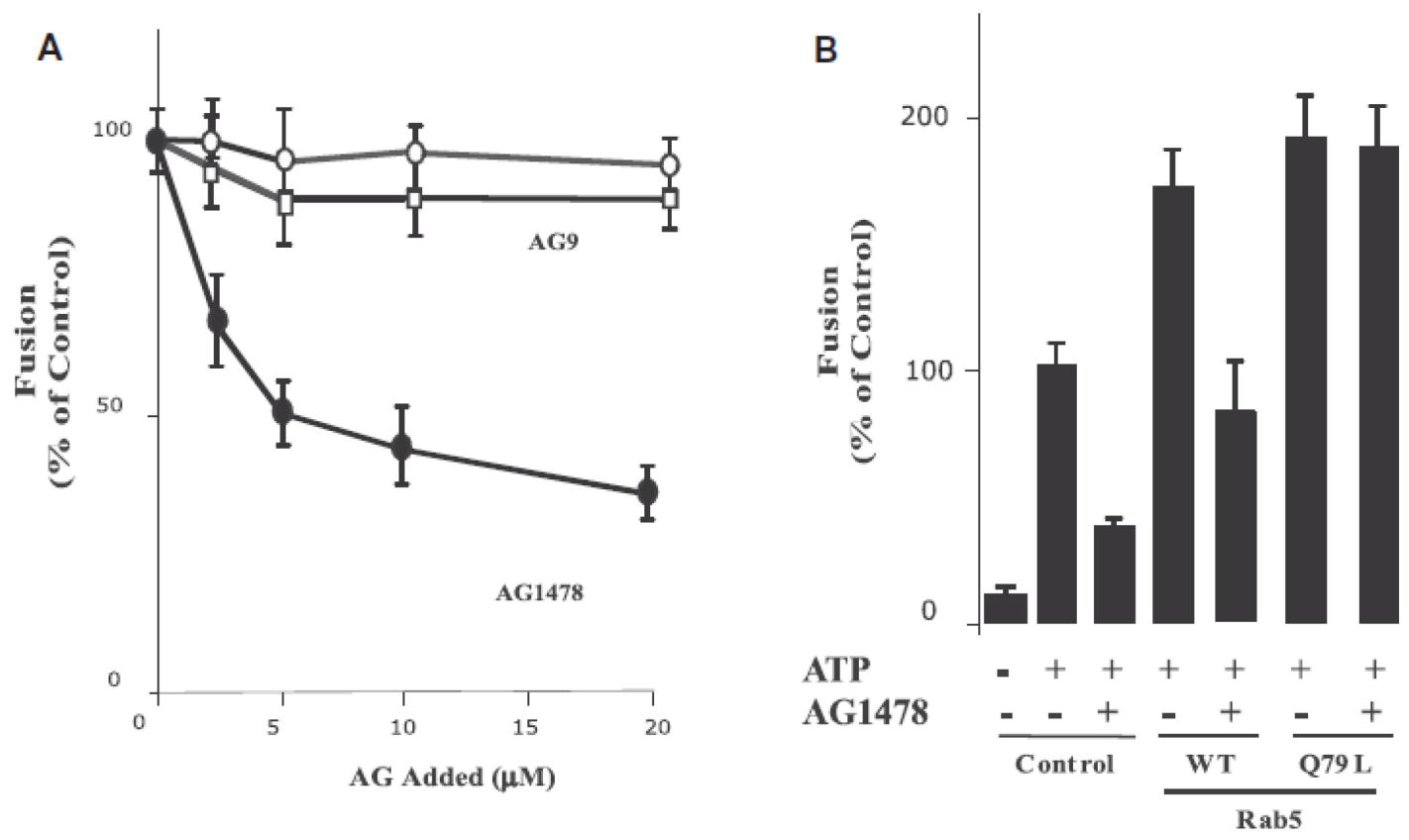

Figure 22. Effect of AG1478 inhibitor on fusion between endosomes. (A) Effect of cytosol and energy on the endosome-endosome fusion. Five-min vesicles containing either Biotin (B)-EGF or Avidin (Av)-GAL were mixed in fusion buffer supplemented with $1 \mathrm{mg} / \mathrm{ml}$ cytosol containing an ATP-regenerating system either in the absence (O) or in the presence of different amounts of AG9)( and AG1478 (•). Samples were then transferred to $37^{\circ} \mathrm{C}$ for the indicated times and processed as described in Materials and Methods in order to determine the percentage forming the immune complex formation. The data are presented as means \pm SD of four independent experiments. (B) Fusion assay was performed under standard conditions as described in Figure 1A, either in the absence or in the presence of Rab5: wild type or Rab5: Q79L mutant, supplemented with either 20 $\mu \mathrm{M}$ AG1478 or $0.5 \mathrm{mg} / \mathrm{ml}$ of cytosol in the presence of ATP. The data are presented as means $\pm \mathrm{SD}$ of four independent experiments. 


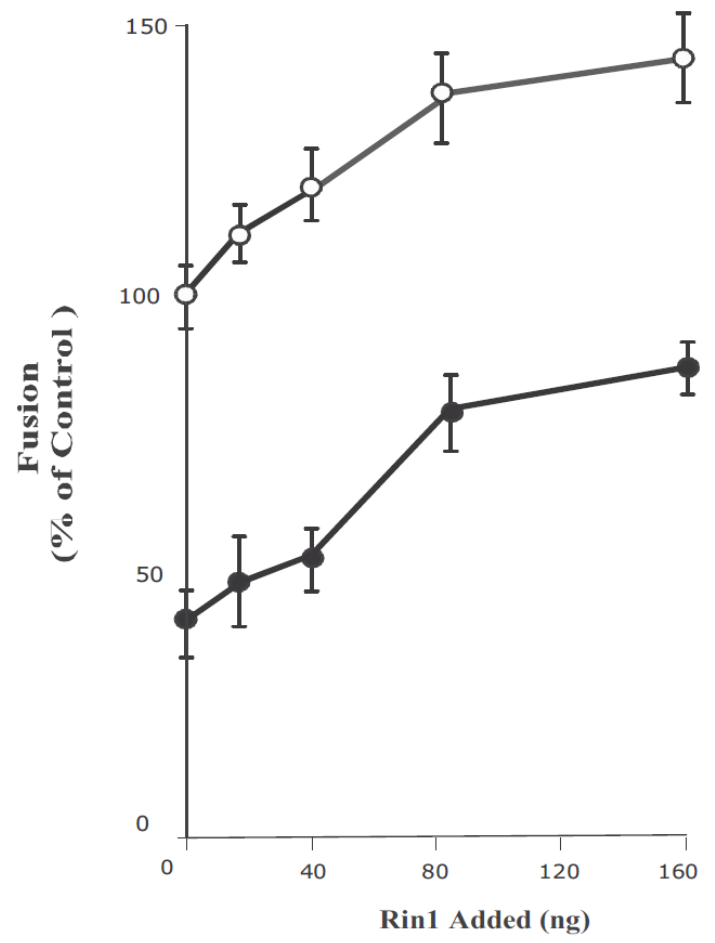

Figure 23. AG1478 blocks endosome fusion stimulated by Rin1. Fusion assay was performed under standard conditions as described in Figure 4, either in the absence or in the presence of different concentrations of Rin1 supplemented with $20 \mu \mathrm{M}$ AG1478 and $0.5 \mathrm{mg} / \mathrm{ml}$ cytosol. Empty cirche (ndicates addit ion of AG9. The data are presented as means $\pm \mathrm{SD}$ of four independent experiments. 


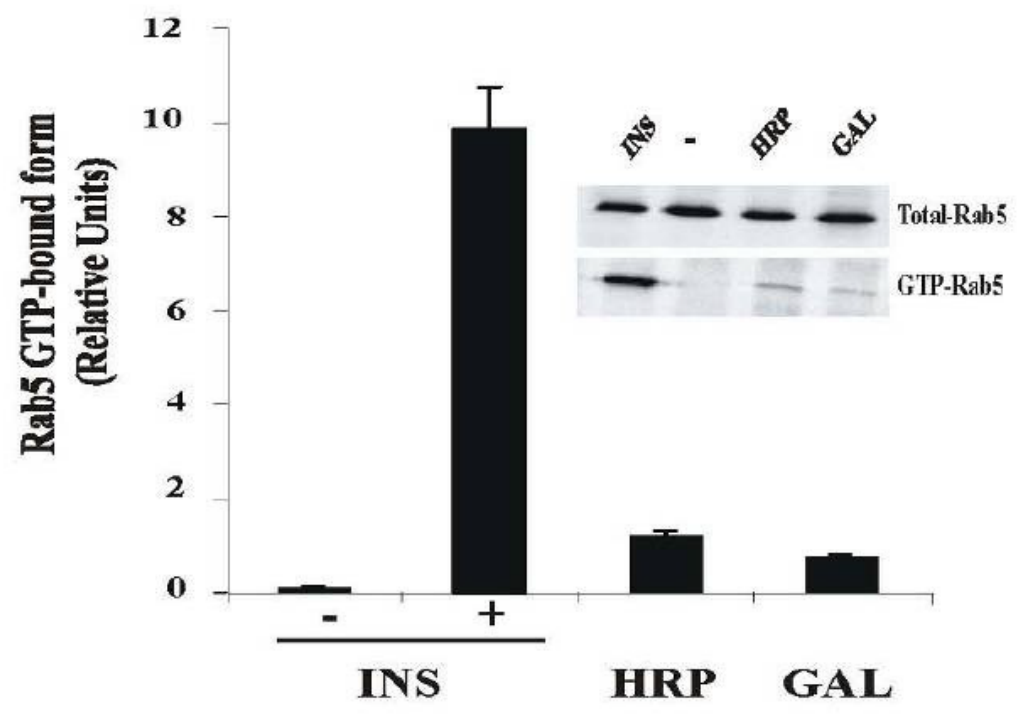

Figure 24. Activation of Rab5 during fluid phase and receptor-mediated endocytosis. NIH-IR cells were transfected with plasmids encoding GFPRab5: wild type in the absence or in the presence of $100 \mathrm{ng} / \mathrm{ml}$ insulin (INS), $2 \mathrm{mg} / \mathrm{ml} \mathrm{HRP} \mathrm{and} 1 \mu \mathrm{g} / \mathrm{ml}$ b-Galactosidase (GAL) as indicated in the Figure. Each ligand was incubated with cells at $4^{\circ} \mathrm{C}$ for $60 \mathrm{~min}$. Cells were then incubated at $37^{\circ} \mathrm{C}$ for $8 \mathrm{~min}$. Subsequently, the cells were washed three times with ice-cold PBS, lysed and incubated with glutathione beads either in the presence of GST-EEA 1 at $4^{\circ} \mathrm{C}$ for $60 \mathrm{~min}$. After incubation, the beads were washed and the presence of activated Rab5 was analyzed by Western blotting using antiRab5 antibodies. The data are presented as means $\pm \mathrm{SD}$ of two independent experiments. 


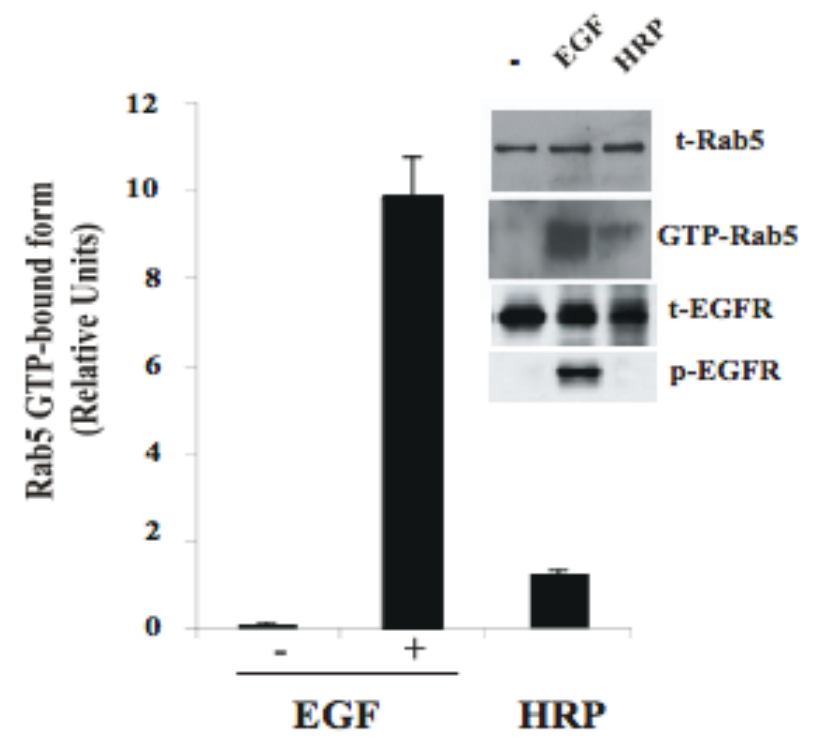

Figure 25. Activation of Rab5 during fluid phase and receptor-mediated endocytosis. NR6 cells expressing EGF-receptor wild type were incubated in the absence or in the presence of $100 \mathrm{ng} / \mathrm{ml} \mathrm{EGF}$ and $2 \mathrm{mg} / \mathrm{ml} \mathrm{HRP}$ as indicated in the Figure. Each ligand was incubated with cells at $4^{\circ} \mathrm{C}$ for $60 \mathrm{~min}$. Cells were then incubated at $37^{\circ} \mathrm{C}$ for 5 min. Subsequently, the cells were washed three times with ice-cold PBS, lysed and incubated with glutathione beads either in the presence of GST alone or GST-EEA1 at $4^{\circ} \mathrm{C}$ for $60 \mathrm{~min}$. After incubation, the beads were washed and the presence of activated Rab5 was analyzed by Western blotting. The data are presented as means $\pm \mathrm{SD}$ of four independent experiments. 


\subsection{References}

Backer J.M., Shoelson S.E., Haring E., White M.F. 1991. Insulin receptors internalize by a rapid, saturable pathway requiring receptor autophosphorylation and an intact juxtamembrane region. J. of Cell Biol. 115: 1535-1545.

Barbieri M.A., Li G., Colombo M.I., Stahl P.D. 1994. Rab5, an early acting endosomal GTPase, supports in vitro endosome fusion without GTP hydrolysis. J. of Biol. Chem. 269: $18720-18722$.

Barbieri M.A., Hoffenberg S., Roberts R., Mukhopadhyay A., Pomrehn A., Dickey B.F.. Stahl P.D. 1998. Evidence for a symmetrical requirement for Rab5-GTP in in vitro endosome-endosome fusion. Journal of Biol. Chem. 273: 25850-25855.

Barbieri M.A, Roberts RL., Gumusboga A., Highfield H., Alvarez-Dominguez C., Wells A., Stahl P.D. 2000. Epidermal growth factor and membrane trafficking. EGF receptor activation of endocytosis requires Rab5a. J. of Cell Biol. 151: 539-550.

Barbieri M.A., Kong C., Chen P.I., Horazdovsky B.F., Stahl P.D. 2003. J. Biol. Chem. 278: $32027-32036$.

Braell W.A. 1987. Fusion between endocytic vesicles in a cell-free system. PNAS USA 84: 1137-1141.

Brandhorst D., Zwilling D., Rizzoli S.O., Lippert U., Lang T., Jahn R. 2006. Homotypic fusion of early endosomes: SNAREs do not determine fusion specificity. PNAS USA 103: 2701-2706.

Bucci C., Parton R.G., Mather I.H., Stunnenberg H., Simons K., Hoflack B., Zerial M. 1992. The small GTPase rab5 functions as a regulatory factor in the early endocytic pathway. Cell 70: 715-728.

Burke P., Schooler K., Wiley H.S. 2001. Regulation of epidermal growth factor receptor signaling by endocytosis and intracellular trafficking. Mol. Biol. Cell 12: 1897-1910.

Carpentier J.L., Paccaud J.P., Gorden P., Rutter W.J., Orci L., 1992. Insulin induced surface redistribution regulates internalization of the insulin receptor and requires its autophosphorylation. PNAS USA 89: 162-166.

Carpentier J.L., Paccaud J.P., Backer J., Gilbert A., Orci L., Kahn C.R., Baecker,- J. 1993. Two steps of insulin receptor internalization depend on different domains of the beta-subunit. J. of Cell Biol. 122: 1243-1252.

Christoforidis S., Miaczynska M., Ashman K., Wilm M., Zhao L., Yip S.C., Waterfield M.D., Backer J.M., Zerial M. 1999. Phosphatidylinositol-3-OH kinases are Rab5 effectors. Nature Cell Biol. 1: 249-252. 
Citri A. \& Yarden Y. 2006. EGF-ERBB signaling: towards the systems level. Nat. Rev. Mol. Cell Biol. 7: 505-516.

Di Guglielmo G.M., Drake P.G., Baass P.C., Authier F., Posner B.I., Bergeron J.J. 1998. Insulin receptor internalization and signalling. Mol. and Cell Biochem 182: 59-63.

Doherty G.J. \& McMahon H.T. 2009. Mechanisms of endocytosis. Annual Review of Biochemistry 78: 857-902 .

Fan J.Y., Carpentier J.L., Gorden P., van Obberghen E., Blackett N.M., Grunfeld C., Orci L. 1982. Receptor-mediated endocytosis of insulin: role of microvilli, coated pits, and coated vesicles. PNAS USA 79: 7788-7791.

Feliciano W.D., Yoshida S., Straight S.W., Swanson J.A. 2011. Coordination of the Rab5 Cycle on Macropinosomes. Traffic 12: 1911-1922.

Fucini R.V., Okada S., Pessin J.E. 1999. Insulin-induced desensitization of extracellular signal-regulated kinase activation results from an inhibition of Raf activity independent of Ras activation and dissociation of the Grb2-SOS complex. J. of Biol. Chem. 274: 18651- 18658.

Galvis A., Balmaceda V., Giambini H., Conde A., Villasana Z., Fornes M.W., Barbieri M.A. 2009. Arch. Biochem. Biophys. 482: 83-95.

Gorvel J.P., Chavrier P., Zerial M., Gruenberg J. 1991. Rab5 controls early endosome fusion in vitro. Cell 64: 915-925.

Gruenberg J.E. \& Howell K.E. 1986. Reconstitution of vesicle fusions occurring in endocytosis with a cell-free system. EMBO 5: 3091-3101.

Hayes N., Howard-Cofield E., Gullick W. 2004. Green fluorescent protein as a tool to study epidermal growth factor receptor function. Cancer Lett. 206: 129-135.

Horiuchi H., Lippe R., McBride H.M., Rubino M., Woodman P., Stenmark H., Rybin V., Wilm M., Ashman K., Mann M., Zerial M. 1997. A novel Rab5 GDP/GTP exchange factor complexed to Rabaptin-5 links nucleotide exchange to effector recruitment and function. Cell 90: 1149-1159.

Huang F. \& Sorkin A. 2005. Mol. Biol. Cell 16: 1268-1281.

Hunker C.M., Giambini H., Galvis A., Hall J., Kruk I., Veisaga M.L., Barbieri M.A. 2006a. Rin1 regulates insulin receptor signal transduction pathways. Experimental Cell Research 312: 1106-1118.

Hunker C.M., Kruk I., Hall J., Giambini H., Veisaga M.L., Barbieri M.A. 2006b. Role of Rab5 in insulin receptor-mediated endocytosis and signaling. Archives of Biochemistry and Biophysics 449: 130-142. 
Khan M.N., Baquiran G., Brule C., Burgess J., Foster B., Bergeron J.J., Posner B.I. 1989. Internalization and activation of the rat liver insulin receptor kinase in vivo. J. of Biol. Chem. 264: 12931-12940.

Klein H.H., Freidenberg G.R., Matthaei S., Olefsky J.M. 1987. Insulin receptor kinase following internalization in isolated rat adipocytes. J. of Biol. Chem. 262: 10557-10564.

Kondratov K.A., Chernorudskiy A.L., Amosova A.P., Kornilova E.S. 2010. Cell Biol. Int. 34: 81-87.

Li G., D'Souza-Schorey C., Barbieri M.A., Roberts R.L., Klippel A., Williams L.T., Stahl P.D. 1995. Evidence for phosphatidylinositol 3-kinase as a regulator of endocytosis via activation of Rab5. PNAS USA. 92: 10207-10211.

Lippe R., Miaczynska M., Rybin V., Runge A., Zerial M. 2001. Functional synergy between Rab5 effector Rabaptin-5 and exchange factor Rabex-5 when physically associated in a complex. Mol. Biol Cell. 12: 2219-2228.

Liu F. \& Roth R.A. 1995. Grb-IR: a SH2-domain-containing protein that binds to the insulin receptor and inhibits its function. PNAS USA 92: 10287-10291.

Maggi D., Andraghetti G., Carpentier J.L., Cordera R. 1998. Cys860 in the extracellular domain of insulin receptor beta-subunit is critical for internalization and signal transduction. Endocrinology 139: 496-504.

Mayorga L.S., Diaz R., Stahl P.D. 1988. Plasma membrane-derived vesicles containing receptor-ligand complexes are fusogenic with early endosomes in a cell-free system. J. of Biol. Chem. 263: 17213-17216.

Mayorga L.S., Diaz R., Colombo M.I., Stahl P.D. 1989. GTP gamma S stimulation of endosome fusion suggests a role for a GTP-binding protein in the priming of vesicles before fusion. Cellular Regulation. 1: 113-124.

McBride H.M., Rybin V., Murphy C., Giner A., Teasdale R., Zerial M. 1999. Oligomeric complexes link Rab5 effectors with NSF and drive membrane fusion via interactions between EEA1 and syntaxin 13. Cell. 98: 377-386.

Mullock B.M. \& Luzio J.P. 1992. Reconstitution of rat liver endosome-lysosome fusion in vitro. Methods. Enzymol. 219: 52-60.

Nielsen E., Christoforidis S., Uttenweiler-Joseph S., Miaczynska M., Dewitte F., Wilm M., Hoflack B., Zerial M. 2000. Rabenosyn-5, a novel Rab5 effector, is complexed with hVPS45 and recruited to endosomes through a FYVE finger domain. J. Cell Biol. 151: 601-612.

O'Bryan J.P., Lambert Q.T., Der C.J. 1998. The src homology 2 and phosphotyrosine binding domains of the $\mathrm{ShcC}$ adaptor protein function as inhibitors of mitogenic signaling by the epidermal growth factor receptor J. Biol. Chem. 273: 20431-20437. 
Paccaud J.P., Siddle K., Carpentier J.L., 1992. Internalization of the human insulin receptor. The insulin-independent pathway. J. of Biol. Chem. 267: 13101-13106.

Parrizas M., Gazit A., Levitzki A., Wertheimer E, LeRoith D. 1997. Specific inhibition of insulin-like growth factor-1 and insulin receptor tyrosine kinase activity and biological function by tyrphostins. Endocrinology. 138: 1427-1433.

Pfeffer S.R. 2007. Unsolved mysteries in membrane traffic. Ann. Rev. Biochem. 76: 629645.

Rubino M., Miaczynska M., Lippe R., Zerial M. 2000. Selective membrane recruitment of EEA1 suggests a role in directional transport of clathrincoated vesicles to early endosomes. J. Biol. Chem. 275: 3745-3748.

Russell D.S., Gherzi R., Johnson E.L., Chou C.K., Rosen O.M. 1987. The proteintyrosine kinase activity of the insulin receptor is necessary for insulinmediated receptor downregulation. J. Biol. Chem. 262: 11833-11840.

Saperstein R., Vicario P.P., Strout H.V., Brady E., Slater E.E., Greenlee W.J., Ondeyka D.L., Patchett A.A., Hangauer D.G. 1989. Design of a selective insulin receptor tyrosine kinase inhibitor and its effect on glucose uptake and metabolism in intact cells. Biochem. 28: 5694-5701.

Scholz H., Baier W., Ratcliffe P., Eckardt K., Zapf J., Kurtz A., Bauer C. 1992. Insulinlike growth factors decrease oxygen-regulated erythropoietin production by human hepatoma cells (Hep G2). American Journal of Physiology. 263: C474-479.

Scita G. \& Di Fiore P.P. 2010. The endocytic matrix. Nature. 463: 464-473.

Semerdjieva S., Shortt B., Maxwell E., Singh S., Fonarev P., Hansen J., Schiavo G., Grant B.D., Smythe E. 2008. Coordinated regulation of AP2 uncoating from clathrincoated vesicles by rab5 and hRME-6. J. Cell Biol. 183: 499-511.

Siemeister G., al-Hasani H., Klein H.W., Kellner S., Streicher R., Krone W., MullerWieland D. 1995. Recombinant human insulin receptor substrate-1 protein. Tyrosine phosphorylation and in vitro binding of insulin receptor kinase. J. Biol. Chem. 270: 48704874.

Simonsen A., Lippe R., Christoforidis S., Gaullier J.M., Brech A., Callaghan J., Toh B.H., Murphy C., Zerial M., Stenmark H. 1998. EEA1 links PI(3)K function to Rab5 regulation of endosome fusion. Nature. 394: 494-498.

Smith R.M. \& Jarett L. 1990. Differences in adenosine triphosphate dependency of receptor-mediated endocytosis of alpha 2-macroglobulin and insulin correlate with separate routes of ligand-receptor complex internalization. Endocrin. 126: 1551-1560.

Sorkin A. \& von Zastrow M. 2009. Endocytosis and signalling: intertwining molecular networks. Nat. Rev. Mol. Cell Biol. 10: 609-622. 
Sorkina T., Huang F., Beguinot L., Sorkin A. 2002. Effect of tyrosine kinase inhibitors on clathrin-coated pit recruitment internalization of epidermal growth factor receptor. $J$. Biol. Chem. 277: 27433-27441.

Tall G.G., Barbieri M.A., Stahl P.D., Horazdovsky B.F. 2001. Ras-activated endocytosis is mediated by the Rab5 guanine nucleotide exchange activity of RIN1. Dev. Cell 1: 7382.

Thomas C.Y., M. Chouinard, M. Cox, S. Parsons, M. Stallings-Mann, R. Garcia, R Jove, R. Wharen. 2003. Int. J. Cancer. 104: 19-27.

Waters S.B., Yamauchi K., Pessin J.E. 1995. Insulin-stimulated disassociation of the SOS-Grb2 complex. Mol. Cell Bioch. 15: 2791-2799.

Wells A., Ware M.F., Allen F.D., Lauffenburger D.A. 1999. Shaping Up for Shipping Out: PLC g Signaling of Morphology Changes in EGF-Stimulated Fibroblast Migration Cell Motil. Cytoskel. 44: 227-233.

Wessling-Resnick M. \& Braell W.A. 1990. The sorting and segregation mechanism of the endocytic pathway is functional in a cell-free system. J. Biol. Chem. 265: 690-699.

Wiley H.S. 2003. Trafficking of the ErbB receptors and its influence on signaling. Exp. Cell Res. 284: 78-88.

Wolff M., Tetzlaff K., Nivens M.C., Schneider F.J., Jung B., Hohlfeld J., Heilker R.. 2011. In vivo inhibition of epidermal growth factor receptor autophosphorylation prevents receptor internalization. Exp. Cell Res. 317:42-50.

Yamamoto N., Mammadova G., Song R.X., Fukami Y., Sato K. 2006. Tyrosine phosphorylation of p145met mediated by EGFR and Src is required for serumindependent survival of human bladder carcinoma cells. J. Cell Sci. 119: 4623-4633. 
CHAPTER 3

RAB5 CAN BE ACTIVATED THROUGH AT LEAST THREE DISTINCT ENDOCYTIC PATHWAYS 


\subsection{Abstract}

The process of endocytosis is a complex pattern of membrane vesicle trafficking, which permits the continuous flow of membrane between cell surface and various intracellular compartments, via fusion and fission events, with Rab5 mediating the fusion events of early endosomes. Since it has been well established that varying concentrations of Epidermal Growth Factor (EGF) during the endocytosis of EGF Receptor (EGFR) can elicit uptake via numerous endocytic routes, we were interested in analyzing which of these endocytic pathways is/are responsible for activation of Rab5. Rab5 is activated in an EGF concentration dependent manner (from $2 \mathrm{ng} / \mathrm{ml}$ to $500 \mathrm{ng} / \mathrm{ml}$ ), plateauing at $100 \mathrm{ng} / \mathrm{ml}$. It was also observed that if cells are stimulated with low quantities of EGF ( $2 \mathrm{ng} / \mathrm{ml})$, temporal activation of Rab5 peaks at about 2.5 minutes and diminishes within 10 minutes. However, if cells are stimulated with high concentration of EGF $(>100 \mathrm{ng} / \mathrm{ml})$, the temporal activation of Rab5 is both heightened as well as prolonged, where even 10 minutes after stimulation, relative activity of Rab5 is much higher than at the maximum point during stimulation with low concentration of EGF. Furthermore, by utilizing specific inhibitors against key regulators of endocytosis and by varying the concentration of EGF stimulation, I have provided evidence for the existence of at least three distinct pathways of Rab5-dependent internalization of growth factor receptors. However, overexpression of either Rin1 or Rabex-5 is able to rescue Rab5 activity even in absence of EGF and/or in the presence of any of the employed inhibitors. Taken together, these results constitute evidence showing selective activation of Rab5 with respect to the different routes of uptake of EGFR. 


\subsection{Introduction}

Cellular membranes allow for compartmentalization of cellular chemistry by generating precise accumulation of proteins on their surfaces or by generating diffusion barriers between lumina and cytoplasm. Variations in the distribution, protein and lipid structure, as well as the luminal makeup of the cellular membranes through the very dynamic fission and fusion reactions that transpire from and between such cellular compartments, are chief regulators of most, extra-nuclear cell biological processes. Such is endocytosis, a process by which cells internalize extracellular molecules, and in doing so control lipid and protein composition of the plasma membrane for the ultimate purpose of regulating cellular interaction with its environment. Depending on the type of cargo that is to be internalized, this can mean regulating expression of cell surface proteins, sampling the cell's surroundings for development and guidance cues, transporting nutrients into cells, regulating the activation of signaling pathways, rescuing proteins placed after vesicle fusion or turning over membrane components by degradation in lysozymes [Doherty and McMahon, 2009; McMahon \& Boucrot, 2011]. On the other hand, pathogens often try to exploit cell's endocytic machinery in order to mediate their internalization. There are numerous routes of endocytosis mobilized by cells. Among others, these are Clathrin Mediated Endocytosis (CME) [Roth \& Porter, 1964], Caveolin Mediated Endocytosis (CavME) [Rothberg et al, 1992], those independent of clathrin and caveolin (CLIC/GEEC type [Kirkham \& Parton 2005; Mayor \& Pagano, 2007], flotillin associated [Frick et al., 2007], macropinocytosis [Shao et al., 2002] and entosis [Overholtzer et al., 2007]). 
CME, the most studied and best characterized pathway of endocytosis, proceeds through initiation, selection of cargo, assembly of the coat, scission and uncoating of the vesicle. Although the major protein which makes up the lattice-like coat around the vesicles, clathrin, does not directly bind to the membrane or cargo receptors, adaptor proteins (such as AP2) and accessory proteins (such as epsin and AP180) are recruited to the plasma membrane in order to form the clathrin coat, all of which are recycled for reuse in another cycle of endocytosis once the vesicle has formed [McMahon \& Boucrot, 2011].

Even though the endocytosis of cargo receptors can be stimulated by ligand binding (as in the case of EGFR, epidermal growth factor receptor), some receptors (such as the transferrin receptor, TfR) are constitutively internalized [Hopkins et al., 1985]. Upon internalization, cargo is sorted in endosomes prior to either being recycled back to the cell surface, or degraded by the lysosome by fusing with the late endosome and the multivesicular body, (MVB) [Grant \& Donaldson, 2009].

Chlorpromazine hydrochloride is a cationic amphipathic chemical which has been extensively used as a potent inhibitor of the clathrin mediated pathway since it inhibits clathrin assembly at the plasma membrane. It should however be noted that that by doing so, it does not affect uptake through caveolin/lipid raft type endocytic pathways [Wang et al., 1993; Rejman et al., 2005]. On the other hand, since chlorpromazine is an amphipathic molecule, it is able to interfere with the biogenesis of large intracellular vesicles, such as micropinosomes, probably due to the fact that it can insert itself into the lipid bilayer and hence increase fluidity of the plasma membrane. This in turn can inhibit formation of membrane invaginations as seen during macropinocytosis. Chlorpromazine 
has also been shown to inhibit function of Phospholipase C (PLC) which has previously been shown to be an important regulator of actin dynamics and therefore also macropinocytosis [Ogiso et al., 1981; Walenga et al., 1981; Giocondi et al., 1995; Wells et al., 1999; Ivanov 2008].

Although CME has been the most widely studied pathway and the best characterized pathwy, recently, it has been shown that cargo can be internalized via numerous clathin independent pathways, also referred to as CIs or Clathrin Indepdendent pathways. [Mayor \& Pagano, 2007]. Moreover, these CIs constitute for approximately three times as much volume of fluid and bulk membrane endocytosed compared to the canonical CME [Howes et al., 2010]. Hence, the study of CIs is becoming progressively more important, but also more complex because these pathways have been shown to be quite diverse as shown in Figure 1.

There is a question of which GTPase is used in the scission of the vesicle from the cell membrane. Some CIs, such as the Caveolin Mediated pathway (CavME) require dynamin (just as the CME pathway), whereas others do not (Clathrin and caveolin independent carrier proteins, also known as CLICs). Furthemore, CLICs can be additionally subdivided by the involvement of various small GTPases (CDC42, RhoA or ARF6) in their respective endocytic pathway(s) [Qualmann \& Mellor, 2006; D'SouzaSchorey \& Cavrier, 2006; Mayor \& Pagano, 2007]. Although the aforementioned small GTPases have been experimentally shown to be involved in their respective pathways (via mutants), it should also be noted that this does not necessarily meant that the small GTPases are obligatory for that particular pathway. 
Caveolin mediated dynamin dependent pathway is the most studied and best characterized CI route of entry. As previously mentioned, CavME is dependent on dynamin and caveolin (the coat that will form around the invagination of the soon to be vesicle) in areas of the membrane where there is an abundance of sphingolipids, cholesterol, signaling proteins and GPI-APs (glycosyl phosphatidylinositol-anchored proteins) [Simons \& Ikonen, 1997; Parton \& Simons, 2007]. CavME pathways also have very diverse cargo, ranging from lipids, proteins and lipid anchored proteins. To pathogens, however endocytosis of these cargo is not subject just to CavME, but other CI pathways as well, which in turn points to the redundancy of endocytic mechanisms for certain molecules [Mayor \& Pagano, 2007]. Another dynamin dependent but clathrin independent pathway of endocytosis is the one regulated by the small GTPase RhoA which has been shown to be responsible for the internalization of the B-chain of the interleukin-2 receptor (IL-2R- $\beta$ ) as well as other protein s (such as $\gamma$-c-cytokine and IgE receptors) in immune cells and fibroblasts [Lamaze et al., 2001].

Filipin, from the polyene family of antibiotics, is a specific inhibitor of the caveolin mediated pathway which leaves the clathrin mediated uptake unaffected [Schnitzer et al., 1994; Orlandi et al., 1998; Rejman et al., 2005]. It has been shown to sequester cholesterol from membrane structures by creating aggregates in the membrane that accumulate cholesterol which in turn leads to profound changes in structure and function of cholesterol rich membrane domains [Orlandi \& Fishman 1998; Ros-Baro et al., 2001; Ivanov 2008]. Another very useful tool in assessing mechanism of dynamin dependent pathways is the specific inhibitor dynamin called dynasore [3-Hydroxy-naphthalene-2- 
carboxylic acid (3,4-dihydroxy-benzylidene)-hydrazide hydrate]. Dynasore inhibits the GTPase activity of dynamin 1 and dynamin2 and in doing so arrests the formation of endocytic clathrin coated pits and vesicles, but has no effect on dynamin independent cellular processes [Macia et al., 2006]. Hence by utilizing a combination of chlorpromazine, dynasore, and filipin, in combination with siRNA against their respective targets, one could gain great insight into the various mechanisms of transport of particular cargo by the aforementioned pathways of endocytosis.

On the other hand, cells can also utilize pathways independent of both clathrin and dynamin and hence also of caveolin. These CLIC pathways were first discovered by observing an increase in fluid-phase endocytosis when expression of dynamin was knocked down [Damke et al., 1995]. However, they are dependent on one of the two small GTPases, either CDC42 or ARF6 from the Rho and Arf family of GTPases respectively. Furthermore, it has been well established that these CLIC pathways, in addition to being independent of dynamin are enriched in GPI-anchoring proteins, sensitive to cholesterol depletion, and contribute a significant fraction of $\mathrm{CTxB}$ (Cholera Toxini subunit B), ricin and fluid internalization [Sabharanjak et al., 2002; Kirkham et al., 2005; Chadda et al., 2007; Kumari \& Mayor, 2008; Howes et al., 2010].

Endocytosis of the Epidermal Growth Factor Receptor (EGFR) is one of the most well characterized models for studying the morphology, kinetics and mechanisms of endocytic pathways, and is a prototypic model for the endocytosis of other Receptor Tyrosine Kinases (RTKs) (Figure 27). The first comprehensive study on EGF endocytosis was described by Carpenter and Cohen [1976], in which many of the key 
concepts of internalization and lysosomal degradation of EGF have been established, which still remains as the foundation of our current understanding of EGFR endocytosis. EGFR is also the most popular model used to study the interplay between endocytosis and signaling. After internalization, EGF and EGFR are efficiently degraded, leading to a significant decrease in the half-life of the EGFR protein [Stonscheck \& Carpenter, 1984], while accelerated internalization and degradation of activated EGFR leads to EGFinduced down regulation of EGFR. Therefore, control of intensity and duration of receptor signaling is mainly regulated via the negative feedback regulatory mechanism of EGFR [Wells et al., 1990]. Basal turnover rates of non-stimulated EGFR reciprocally correlate with their expression levels. This is most likely due to the saturation of the internalization and degradation steps of trafficking. Furthermore, it has also been shown that the binding of EGF to EGFR results in acceleration of receptor internalization. This is due to the internalization of EGFR complexes through clathrin coated pits [Wiley et al., 1991] because overexpression of dominant-negative mutants as well as RNA interference proteins essential for CME inhibited EGFR internalization [Damke et al., 1995; Motley et al., 2003; Huang et al., 2004; Sorkin \& Goh, 2008]. Hence, both lines of evidence strongly argue that CME is the major pathway of EGFR internalization.

Interestingly, high internalization rates of EGFR, that are characteristic of CME, were observed only when EGF was used in low, physiological concentrations $(\geq 1-2 \mathrm{ng} / \mathrm{ml})$, whereas the apparent rate of EGF uptake was decreased with increasing EGF concentrations. Based on such a saturation of the internalization process, the Wiley et al. proposed that the presence of high concentrations of EGF-receptor complex at the cell surface limits the capacity and overwhelms the rapid internalization pathway [Wiley, 
1988; Lund et al., 1990]. More recently, it was shown that the internalization of high EGF concentrations was only minimally affected by overexpression of the dominant negative dynamin mutant or by knockdown of clathrin heavy chain by siRNA, both of which have been previously shown to significantly impede the CME pathway at low concentrations of EGF [Jiang \& Sorkin, 2003; Sigismund et al., 2005]. Hence, under physiological conditions (low ligand concentrations and moderate expression levels of EGFR), EGFR is internalized mostly by CME, whereas under conditions of receptor overexpression and/or high ligand concentrations, the CIs determine(s) the uptake of EGFR into the cell [Sorkin \& Goh, 2008].

In addition to CME, in cells where EGFR activation leads to increased membrane dynamics, ruffling and pinocytosis, EGFR complexes can enter the pinocytic vesicles and ruffle-generated endocytic compartments. However, in other cells, activated EGFR can be taken up by the mechanisms sensitive to cholesterol-disrupting drugs as well as through CavME pathways. Although they may have faster kinetics as compared to the constitutive receptor internalization, all these CI pathways are significantly slower than CME.

The lack of specific inhibitors of clathrin-independent endocytosis makes it problematic to explain the mechanisms and assess the significance of these pathways in EGFR internalization [Sorkin \& Goh, 2008]. Previous studies have also shown that overexpression of Rab5 enhances EGF-stimulated endocytosis of EGFR, whereas the dominant negative form of Rab5 (S34N) represses these processes [Barbieri et al., 2000; Dinneen \& Ceresa 2004]. Furthermore, Rab5: S34N substantially inhibits degradation of EGFR, whereas continual expression of Rab5: Q79L (the constitutively active form) 
causes a ligand-independent internalization of EGFR into intracellular vesicles [Barbieri et al., 2004; Chen et al., 2009].

Here, I have taken advantage of the well-characterized trafficking pathways of the EGF receptor, in order to assay the differential activation of Rab5. We show that if cells are stimulated with low quantities of EGF [2ng/ml], temporal activation of Rab5 peaks at about 2.5 minutes and diminishes within 10 minutes. However, when cells are stimulated with high concentrations of EGF [ $>100 \mathrm{ng} / \mathrm{ml}]$, the temporal activation of Rab5 is both heightened as well as prolonged, where even 10 minutes after stimulation, relative activity of Rab5 is much higher than at the maximum point during stimulation with low concentration of EGF. Furthermore, by utilizing specific inhibitors against key regulators of endocytosis (Dynasore hydrate, Chlorpromazine hydrochloride and Filipin) and by varying the concentration of EGF stimulation, we have provided first evidence for the existence of at least three distinct pathways of Rab5-dependent internalization of growth factor receptors. However, overexpression of either Rin1 or Rabex-5 is able to rescue Rab5 activity even in absence of EGF and/or in the presence of any of the employed inhibitors. Taken together, these results constitute evidence showing selective activation of Rab5 with respect to the different routes of uptake of EGFR as well as differential activation of Rab5 by three Rabex-5 isoforms. 


\subsection{Materials \& Methods}

\subsubsection{Cell Culture \& Materials}

NR6 cell line overexpressing the human EGF receptor (NR6-E) was grown to confluence in Dulbecco's modified Eagle's medium supplemented with 5\% fetal bovine serum and 200ug/ml G418. Rab5, Rabex-5, $\alpha$-Tubulin and phospho-EGFR antibodies were from Cell Signaling Technology (Beverly, MA). Rin1 antibody was purchased from Abcam Inc and total EGFR antibody was from Santa Cruz Biotechnology Inc (Santa Cruz, CA). EGF was purchased from Merk KGaA (Darmstadt, Germany). All HRP labeled secondary antibodies were purchased from Jackson ImmunoResearch Laboratories (West Grove, PA) while all Alexa-conjugated secondary antibodies were purchased from Life Technologies (Grand Island, NY). Chlorpromazine, dynasore hydrate and filipin were obtained from Sigma (St. Louis, MO). All other chemicals were obtained from Sigma, unless otherwise stated.

\subsubsection{Assessment of Rab5 activity via EEA1 pulldown assay}

NR6-E cells were lysed using a buffer containing $20 \mathrm{mM}$ HEPES (pH 7.5), $100 \mathrm{mM}$ $\mathrm{NaCl}, 1 \mathrm{mM}$ DTT, $5 \mathrm{mM} \mathrm{MgCl} 2,5 \%$ glycerol and $1 \%$ Triton-X-100 supplemented with 1 mM PMSF. Lysates $(0.5 \mathrm{ml})$ were then incubated with $50 \mu 1$ glutathione beads containing $\sim 5 \mu \mathrm{g}$ of GST-EEA 1 at $4^{\circ} \mathrm{C}$ while rocking for $1 \mathrm{hr}$. After incubation, the beads were washed three times using the lysis buffer. The pull-downs were subjected to SDS-PAGE and analyzed by immunoblotting using an anti-Rab5 antibody. 


\subsubsection{Subcellular Fractionation}

NR6-E cells were cultured in $100 \mathrm{~cm}^{2}$ dishes until confluency and then serum starved for $14 \mathrm{hrs}$. After starvation, cells were washed with PBS once, then allowed to bind $100 \mathrm{ng} / \mathrm{ml}$ EGF for $1 \mathrm{hr}$ at $4^{\circ} \mathrm{C}$ after which they the uptake was done at $37^{\circ} \mathrm{C}$ for $5 \mathrm{~min}$. They were then washed again with PBS to remove any unbound ligand and resuspended in binding buffer (PBS, 1mM PMSF, 1mM EDTA, 1mM EGTA, 1mM NaF, $1 \mathrm{mM}$ $\mathrm{Na}_{3} \mathrm{VO}_{4}$ and $1 \mathrm{mM}$ DTT) and lysed by passing through a homogenizer 15 times. The homogenate was centrifuged at $600 \mathrm{x}$ g to pre-clear cell and nuclear debris and then at $75,000 \mathrm{x} \mathrm{g}$ for $1 \mathrm{hr}$ at $4^{\circ} \mathrm{C}$ to separate the cytosol and membrane fractions. Membrane and cytosol fractions were then treated with sample buffer and subject to subsequent western blotting.

\subsubsection{Western Blot analysis}

NR6-E cells were cultured in a 6-well plate in growth medium and then serumstarved for $14 \mathrm{hrs}$. After starvation, cells were washed with PBS and then allowed to bind EGF for $1 \mathrm{hr}$ at $4^{\circ} \mathrm{C}$ and then after which the uptake was done at $37^{\circ} \mathrm{C}$ for the noted time. Cells were then lysed in ice-cold lysis buffer (20 mM Tris- $\mathrm{HCl}(\mathrm{pH} 7.5), 150 \mathrm{mM} \mathrm{NaCl}$ and $1 \%$ Triton $\mathrm{x}-100)$. The lysates were clarified by centrifugation and subjected to SDSPAGE and analyzed by Western blotting using the specific antibodies. Relative Units of proteins were determined by densitometry using the ratio of Rab5, phospho-EGFR, Rin1, or Rabex-5 to total-EGF-receptor and tubulin, respectively. 


\subsubsection{Inhibition of endocytosis}

NR6-E cells were cultured in a 6-well plate in growth media and then serum-starved for $14 \mathrm{hrs}$. After starvation, cells were treated with either $20 \mu \mathrm{M}$ chlorpromazine, $80 \mu \mathrm{M}$ dynasore hydrate, $1 \mathrm{mg} / \mathrm{ml}$ filipin, or a combination of the three, for 30 minutes at $4^{\circ} \mathrm{C}$. EGF was then allowed to bind EGF for $1 \mathrm{hr}$ at $4^{\circ} \mathrm{C}$ and then after which the uptake was done at $37^{\circ} \mathrm{C}$ for 2.5 minutes, after which the cells were then lysed in ice-cold lysis buffer. The lysates were clarified by centrifugation and subjected to SDS-PAGE and analyzed by Western blotting using the specific antibodies.

\subsubsection{Confocal microscopy}

NR6-E cells were seeded on glass coverslips at $1.0 \times 10^{5}$ per well. The following day, cells were transfected using FugeneHD with either pEGFP-Rab5: WT. Cells were then starved in serum-free Dulbecco's modified Eagle's medium supplemented with 5\% BSA Fraction V (MP) the next day overnight, and treated as indicated with $20 \mu \mathrm{M}$ chlorpromazine, $80 \mu \mathrm{M}$ dynasore hydrate, $1 \mathrm{mg} / \mathrm{ml}$ filipin, or a combination of the three, for 30 minutes at $4^{\circ} \mathrm{C}$, after which they were treated with either $2 \mathrm{ng} / \mathrm{ml}$ or $100 \mathrm{ng} / \mathrm{ml}$ EGF for $1 \mathrm{hr}$ at $4^{\circ} \mathrm{C}$ and then stimulated to take up the ligand for $2.5 \mathrm{~min}$ at $37^{\circ} \mathrm{C}$. They were washed with PBS and fixed in 4\% paraformaldehyde for 20 minutes. Fixed cells were permeabilized using $0.1 \%$ Triton $\mathrm{X}-100$ for $20 \mathrm{~min}$ at room temperature and subsequently probed either with the rabbit polyclonal EGF-receptor or Rabex-5 antibodies for $1 \mathrm{hr}$. Secondary antibody used was Alexa594-conjugated goat anti-rabbit IgG. Coverslips were mounted with Prolong and viewed on a Leica TCS SP2 confocal microscope. 


\subsubsection{Image analysis}

Images of fluorescent Rab5-positive endosomes were obtained using Leica TCS software. Endosome size distribution of at least 1252 Rab5wt endosomes from NR6-E cells alone or NR6-E cells overexpressing Rabex-5 constructs (Full length, Long or Short) were analyzed by measuring both endosome diameter and perimeter using NIH Image J software (obtained from http://rsweb.nih.gov/ij/).

\subsection{8 siRNA knockdown of Caveolin, Dynamin, Rin1 and Rabex-5}

In a 6 -well dish, $1-1.5 \times 10^{5}$ cells were plated per well and transfected with $50 \mathrm{pmol}$ of siRNA when cells reached $40-50 \%$ confluency. The sequences of mouse siRNA used were the following: Caveolin1- 5'GGCCAGCUUCACCACCUUCUU3'; Dynamin15'GGAUUUGGCCAACUCUGAU3'; Dynamin2- 5'UUAACCACGCCGAUAUAGC3'; Rabex5- 5'GCUCCCAGUCCUUCCAUAA3'; Rin1 siRNA was purchased as a pool of three specific 20-25nt long siRNAs from Santa Cruz Biotechnology, Inc. (sc-40912).

\subsection{Results}

3.4.1 Activation of Rab5 is dependent on temperature, concentration of ligand and time.

To optimize conditions for activation of Rab5, NR6 cells over-expressing EGFR (NR6-E cells) were allowed to bind various concentrations of EGF $(0,2,50,100$ or $500 \mathrm{ng} / \mathrm{ml}$ ) for one hour at $4^{\circ} \mathrm{C}$, after which they were stimulated to internalize the receptor-ligand complex at $37^{\circ} \mathrm{C}$ for 5 minutes (previously described by Lenhard et al., 1992, as optimal conditions for endosome-endosome fusion) and assayed for activation of Rab5. Because it has already been established that EGFR can be endocytosed via different pathways, depending on the concentration of ligand used, we decided to 
examine activation of Rab5 using various concentrations of EGF. We saw that there was a concentration dependent activation of Rab5 with respect to the amount of EGF employed, which plateaued at 100ng/ml (Figure 27). Because a difference in activation at Low concentration $(2 \mathrm{ng} / \mathrm{ml})$, in comparison to High concentration $(100 \mathrm{ng} / \mathrm{ml})$ was observed, activation of Rab5 was then tested at different time points using both Low and High concentrations of EGF using a similar protocol, where we observed activation of Rab5 after $0,1,2.5,5,10$ and 20 minutes of EGFR uptake. Using Low concentration of EGF, when the clathrin dependent endocytosis of EGFR is predominantly utilized, we see that the optimal Rab5 activation is around 2.5 minutes after stimulation at $37^{\circ} \mathrm{C}$, where it plateaus until $5 \mathrm{~min}$ and then starts to decrease until 10 minutes after stimulation, when do not detect any active Rab5 (Figure 28a \& 28b). On the other hand, when using High EGF, or when clathrin independent uptake of EGFR is predominant, both a heightened as well as prolonged activation of Rab5 was observed in comparison to Low EGF. Upon EGFR uptake, Rab5 activation peaked approximately after 5 minutes of uptake and started to decrease but remained partially detectable after 10 minutes and was undetectable after 20 minutes of uptake (Figure 28a \& 28b). When looking at the phosphorylation of EGFR, the same trend was observed, where Rab5 activation was highly correlated to EGFR phosphorylation. However, some EGFR phosphorylation was detected even 20 minutes after uptake (Figure 28c). This is in agreement with the previous results which associate the tyrosine kinase activity of receptors to activation of Rab5 [Jozic et al., 2011; Jozic et al., 2012]. We then went on to optimize the temperature at which Rab5 activation is ideal. Using low EGF, we observed inhibition of Rab5 activity at both $4^{\circ} \mathrm{C}$ and $17^{\circ} \mathrm{C}$, while no effect at $37^{\circ} \mathrm{C}$ (Figure 29). This is in agreement 
with current understanding of endosome fusion, since at $4^{\circ} \mathrm{C}$ both receptor internalization and endosome fusion are blocked, while at $17^{\circ} \mathrm{C}$ only endosome fusion is blocked and at $37^{\circ} \mathrm{C}$ both internalization or fusion are intact.

\subsubsection{Rab5 can be activated via at least three distinct pathways of EGFR endocytosis.}

Rab5 was differentiatlly activated by low and high concentrations of EGF suggesting that different pathways of activation might be involved. First, NR6-E cells were treated with Chlorpromazine, a specific inhibitor of clathrin (and therefore clathrin mediated endocytosis (CME)), and then stimulated with low or hight EGF to measure Rab5 activation. A significant inhibition of Rab5 activation at low EGF was observed when utilizing the specific inhibitor of CME Chlorpromazine (Figure 30a) which is in accordance to the literature. However, a very minute decrease in activation at high EGF was observed when using Chlorpromazine (Figure 30a), which points to the fact that at low EGF Rab5 is predominantly activated through the CME pathway, but at high EGF, Rab5 can be activated in pathway(s) independent of CME. Specific inhibitors of clathrin independent pathways were then used to better ascertain the contribution of Rab5 activation. Since Filipin is a specific inhibitor of caveolin which does not inhibit or interact with clathrin, when Filipin was utilized in our system, it caused a major reduction of Rab5 activation at high concentration of EGF (attributed to activation of Rab5 via CavME), but no effect at low EGF (Figure $30 \mathrm{~b} \& 31$ ). Since both the caveolin and clathrin mediated pathways can be controlled by dynamin, an inhibitor of dynamin called Dynasore hydrate was employed, and which showed a similar inhibition to Chlorpromazine at low EGF and a more pronounced effect in comparison to Filipin at 
high EGF (Figure 30c \& 31), meaning that there is/are pathway(s) of EGFR uptake independent of CME and CME which can activate Rab5. Because there are no specific inhibitors of other CIs available, other aspects to Rab5 activation could not be further delineated, which altogether points to at least two specific pathways of Rab5 activation and one CI pathway which is independent of dynamin.

Morphology of NR6-E cells indicates a similar story. NR6-E cells were transfected with GFP-Rab5 and treated the cells as described above, but instead of lysing them and using for EEA1 pull-downs, the cells were fixed with paraformaldehyde and examined with anti-EGFR antibodies. In comparison to cells which have not been stimulated with EGF, where Rab5 is localized in very punctuate structures that do not localize with EGFR (Figure 32: A1-A3), if cells are stimulated with either low EGF (Figure 32: B1B3) or high EGF (Figure 32: C1-C3) but without any inhibitor, formation of enlarged Rab5 positive endosomes and co-localization with EGFR were observed (Figures 32: B1C3). However, if Chlorpromazine at low EGF was utilized, a reduction in the number of Rab5 positive endosomes as well as the co-localization with EGFR (Figure 32: D1-D3) was observed, but when high EGF is employed, more internalized EGFR, more colocalization with Rab5 and more enlarged Rab5-positive endosomes were observed (Figures 32: E1-E3). Filipin at Low EGF does not seem to inhibit EGFR internalization, its localization with Rab5 or the formation of Rab5 positive endosomes (Figures 32: H1H3), whereas at High EGF, it decreases the amount of enlarged Rab5-positive endosomes as well as the co-localization of EGFR with Rab5 (Figures 32: I1-I3). 
Utilization of Dynasore seems to have the most effect on both internalization of EGFR as well as the formation of Rab5 positive endosomes and co-localization of EGFR with Rab5 at both Low and High EGF (Figures 32: F1-G3). If we combine Chlorpromazine and Dynasore, we almost completely inhibit both the formation of enlarged Rab5 positive endosomes as well as the internalization of EGFR was inhibited at low EGF (Figures 32: J1-J3), but not at high EGF (Figures 32: K1-K3). Lastly, if Dynasore and Filipin were used, there is an apparent decrease in amount of enlarged Rab5-positive endosomes and some inhibition of EGFR internalization at Low EGF (Figures 32: L1-L3), and a less pronounced inhibition at High EGF (Figures 32: M1-M3), meaning that apart from dynamin mediated CME and CavME, there appear to be other pathways through which Rab5 can mediate endosome-endosome fusion, i.e., enlarged Rab5-positive endosomes.

\subsubsection{Rabex-5 and Rin1 can both rescue activation of Rab5 when one of the pathways} of EGFR internalization is blocked.

Since several pathways of Rab5 activation were established above, the focus of interest switched to understand what was happening to Rab5 GEFs Rabex-5 and Rin1, namely if they had selective effects on specific pathways of EGFR endocytosis. If either Rin1 or Rabex-5 were overexpressed, no matter which pathway we try to inhibit, activation of Rab5 was rescued (Figure 33). On the other hand, if Rabex-5 is knocked down using siRNA, no detectable Rab5:GTP was observed when stimulated by either low or high EGF (Figure 34), meaning that Rabex-5 must play an essential role in activation of Rab5, regardless which pathway of EGFR endocytosis is employed. 


\subsubsection{Rabex-5 splice variants selectively activate Rab5.}

Because Rabex-5 knockdown has a debilitating effect on activation or Rab5 and since there are three naturally occurring splice variants of Rabex-5, the ability to selectively of these three splice variants to selectively activate Rab5 was examined. As shown in Figure $35 \mathrm{a}$, the splice variant which has been extensively used to characterize structure and function of Rabex-5 by various groups (termed here Rabex-5: Full Length or Rabex-5: FL). Furthermore, our laboratory has discovered two other splice variants from human placenta which we have termed Rabex-5: Short (missing 31 amino acids between the MIU and the MBM domains and the terminal 8 amino acids of the VPS9 domain in comparison to Rabex-5: FL) and Rabex-5: Long (contains extra 139 amino acids at the N terminus as well as 38 amino acids extra in the MBM domain in comparison to Rabex-5: FL).

First, each splice variant was overexpessed in NR6-E cells and activation of Rab5 using 0, low or high EGF was examined as previously described. As shown in Figure $35 \mathrm{~b}$, all three splice variants were able to activate Rab5 without stimulation by EGF, however, Rabex-5: Short isoform activated Rab5 somewhat less (Figure 35b). Second, when GFP-Rab5 was transfected into cells expressing the Rabex-5 isoforms, it was observed that both Long and Short splice variants do not have much of an effect at low EGF but they do have a significant impact on formation of Rab5-positive endosomes when high EGF was utilized. This is further accented by a higher Pearson coefficient of correlation for each condition when cells were stimulated by high EGF (Figure 36). Third, when the perimeter of endosomes under each condition was compared, a decrease in the number of cells with an enlarged endosome perimeter for Rabex-5: Short splice 
variant in comparison to either Rabex-5: FL or Rabex-5: Long splice variants were observed (Figure 37). Last, subcellular distribution of Rabex-5 splice variants was compared upon stimulation by differing concentrations of EGF. For Rabex-5: FL and Rabex-5: Long splice variants, as EGF was added to the cells, Rabex-5 started to accumulate in the membrane fraction with High EGF attributing much more to the membrane fraction than low EGF (Figure 38). Alternatively, for Rabex-5: Short splice variant, although the distribution of membrane to cytosol at low EGF is similar to what was previously observed with Rabex-5: FL and Rabex-5: Long, at high EGF there is not as much of a shift from the cytosol to the membrane fraction (Figure 38).

\subsection{Discussion}

Endocytosis of EGFR has been a well characterized process which commences with ligand stimulation, proceeds with ligand-receptor complex internalization, fusion with early endosome and then either degradation in the lysosome or recycling back to the cell surface. Since it has been well established that varying concentrations of EGF during the endocytosis of EGFR can elicit uptake of the ligand-receptor complex via numerous endocytic routes [Damke et al., 1995; Jiang \& Sorkin, 2003; Sorkin \& Goh, 2008] and given that Rab5 has been termed as the master regulator of endosome biogenesis [Zerial \& McBride, 2001; Zeigerer et al., 2012] elucidating the role of this small GTPase could be essential to understanding the mechanism of each of these endocytic routes. Our previous work has already shown that the tyrosine kinase activity of RTKs (namely EGFR and IR) is closely tied to activation of Rab5 in mouse fibroblast NR6 cells and human hepatocyte HepG2 cells respectively [Jozic et al., 2011; Jozic et al., 2012]. 
Here I show that activation of Rab5 is dependent on temperature, time and concentration of ligand available to the receptor at the plasma membrane which can directly be correlated to the tyrosine phosphorylation of EGFR. As Sorkin's group has shown, although pathways which internalize high quantity of ligand/receptor may have faster kinetics as compared to the constitutive receptor internalization, all these CI pathways are significantly slower than CME [Sorkin \& Goh, 2008]. Hence, it should be of no surprise that we were able to detect active Rab5 even 10 minutes after the uptake of receptors had commenced when high concentration of EGF was utilized (Figure 30b). Although varying the concentration of ligand elicits numerous pathways of EGFR endocytosis, it was previously shown that inhibition of either dynamin or clathrin (by overexpression of dominant negative mutants or by silencing via siRNA) can inhibit CME dependent uptake of EGFR (when availability of EGF is low), while having minimal effects on EGFR dependent endocytosis when availability of EGF is High [Jiang \& Sorkin, 2003; Sigismund et al., 2005]. By utilizing this knowledge, I employed specific potent inhibitors against clathrin (chlorpromazine), dynamin (dynasore) and caveolin (filipin) to understand how inhibition of each pathway may affect activation of Rab5. What we found was that Rab5 may be activated via at least three different pathway of EGFR endocytosis.

First, I confirmed that Rab5 can be activated through CME. When the availability of EGF at the plasma membrane is Low or when the primary pathway of EGFR endocytosis is through the canonical CME, inhibition of this pathway should also significantly inhibit activation of Rab5, which was previously observed when chlorpromazine was used to inhibit CME (Figure 30a). Furthermore, because the contribution of CME to endocytosis 
of EGFR when the availability of EGF is high should be minimal, inhibition of CME during this time should also have minimal impact on activation of Rab5, which was previously observed (Figure 30a). Formation of enlarged Rab5 positive endosomes in intact cells (which are indicative of endosome-endosome fusion and therefore activation of Rab5) where utilization of chlorpromazine at Low EGF significantly retarded the formation these enlarged endosomes (as well as inhibited co-localization with EGFR on the internalized since there was no internalization via the clathrin coated vesicle), while having very miniscule effect when high EGF was employed or when the contribution of CME is very minimal (compare Figure 32: D1-D3 to Figure 32: E1-E3).

Second, inhibition of caveolin (by using filipin) when the predominant route of endocytosis is through CME (when stimulation of EGF is low), should not perturb activation of Rab5 significantly and it does not (Figure 30b). However, since CavME has some contribution to the clathrin independent endocytosis of EGFR when the concentration of EGF is high, it should be of no surprise that inhibiting caveolin during this time also has an impact on activation of Rab5 (Figure 30b). Similarly, when comparing formation of enlarged Rab5-positive endosomes in intact cells, we saw that filipin had very little impact when low EGF (when mainly CME is taking place) was used in comparison when High EGF was used and when some CavME is taking place (compare Figure 32: H1-H3 to Figure 32: I1-I3).

Lastly, however, Rab5 can be activated when both CME and CavME pathways are inhibited. Since dynamin is an integral part of both CavME and CME, utilization of dynasore (a specific inhibitor of dynamin) and high EGF has introduced at least a third potential pathway through which Rab5 can be activated through (Figure 30c). However, 
because there are no commercially available specific inhibitors of CLIC pathways, discerning which other pathway(s) can contribute to activation of Rab5 when there is high concentration of EGF available at the cell surface. A similar result was observed when the effect that dynasore had on formation of enlarged Rab5-positive endosomes in intact cells was compared during low and high EGF availability (Figure 32: F1-F3 to Figure 32: G1-G3).

To further understand activation of Rab5 during Low or High ligand availability, the effect of overexpression or depletion of some GEFs (namely Rabex-5 and Rin1) was assayed on activation of Rab5. Overexpression of either GEF even without presence of any ligand at all or in presence of any of the aforementioned inhibitors, caused activation of Rab5 (Figure 33). However, any stimulation with either Low or High EGF increases the amount of active Rab5, though there does not to be a significant difference between the two ways of stimulation or between any of the previously mentioned pathways of endocytosis. On the other hand, depletion of either GEF by using siRNA prevents activation of Rab5 via either pathway of stimulation meaning that both Rabex-5 and Rin1 both play essential roles in activation of Rab5, regardless of which pathway of endocytosis is employed (Figure 34).

Since our lab has discovered the presence two splice variants of Rabex-5 (which we have termed Rabex-5: Short and Rabex-5: Long) in addition to the one which has previously been described in literature (which we have termed Rabex-5: FL), the effect these splice variants had on activation of Rab5 was assessed with respect to availability of ligand at the plasma membrane. As shown in Figure 35a, the splice variant which has been extensively used to characterize structure and function of Rabex-5 is termed here 
Rabex-5: Full Length (or Rabex-5: FL), in addition to the Rabex-5: Short splice variant (because it is missing 39 amino acids in comparison to Rabex-5: FL) and Rabex-5: Long splice variant (which contains extra 177 extra amino acids in comparison to Rabex-5: FL). Of the missing amino acids which are absent in Rabex-5: Short, 31 are in the region between the MIU (Motif Interacting with Ubiquitin) and MBM (Mebrane Bound Motif) motifs and 8 are in the terminal region of Vacuolar sorting protein 9 (Vps9) domain, hence it is not surprising that in in comparison to Rabex-5: FL and Rabex-5: Long, Rabex-5: Short splice variant is not as efficient in activating Rab5 in absence of EGF (Figure 35b). When I compared the co-localization of Rab5 with Rabex-5 (either FL, Long or Short) in intact cells I saw that when there is no EGF or low concentration of EGF available, the Short splice variant (and to some the extent Long splice variant at Low EGF) poorly co-localize with Rab5 in comparison to Rabex-5: FL. On the other hand, when high concentration of EGF was available to the cells, the Long (and to a lesser extent the Short splice variant) co-localize much better with Rab5 (Figure 36). This could mean that the three spice variants are evolutionarily conserved to be redundant as it has been shown to be the case with other small GTPases. Furthermore, if endosome size of cells is compared to those that were overexpressing one of the three splice variants of Rabex-5 when not stimulated with EGF or when stimulated with low/high concentrations of EGF to cells expressing endogenous levels of Rabex-5: FL, a significant decrease in size of the Rabex-5: Short splice variant expressing cells was observed (Figure 37). Subcellular fractionation of cell lines overexpressing each splice variant shows a similar pattern as both Rabex-5: FL and Rabex-5: Long spice variants show accumulation of Rabex-5 in the membrane fraction for Low and High EGF (Figure 38). On the other 
hand, for Rabex-5: Short splice variant, there did not appear to be much of a change at high EGF between cytosol and membrane fractions (Figure 38), giving further proof to our initial observation that Rabex-5: Short splice variant may not be necessary or able to activate Rab5 during endocytosis of EGFR at high concentrations of EGF.

In conclusion, I have taken advantage of the well-characterized trafficking pathways of the EGF receptor, in order to assay the differential activation of Rab5. I have shown that activation of Rab5 is dependent on temperature, time of uptake as well as the concentration of ligand available at the cell surface. Furthermore, by utilizing specific inhibitors against key regulators of endocytosis (Dynasore hydrate, Chlorpromazine hydrochloride and Filipin) and by varying the concentration of EGF stimulation, I have provided evidence for the existence of at least three distinct pathways of Rab5-dependent internalization of growth factor receptors, however, due to the lack of availability of specific inhibitors of other less common endocytic pathways, further discrimination of Rab5 activation can not be determined. On the other hand, overexpression of either Rin1 or Rabex-5 is able to rescue Rab5 activity even in absence of EGF and/or in the presence of any of the employed inhibitors, but that Rabex-5: Short splice variant was not as good in activating Rab5. Taken together, these results constitute evidence for the first time showing selective activation of Rab5 with respect to the different routes of uptake of EGFR as well as differential activation of Rab5 by three Rabex- 5 splice variants. 


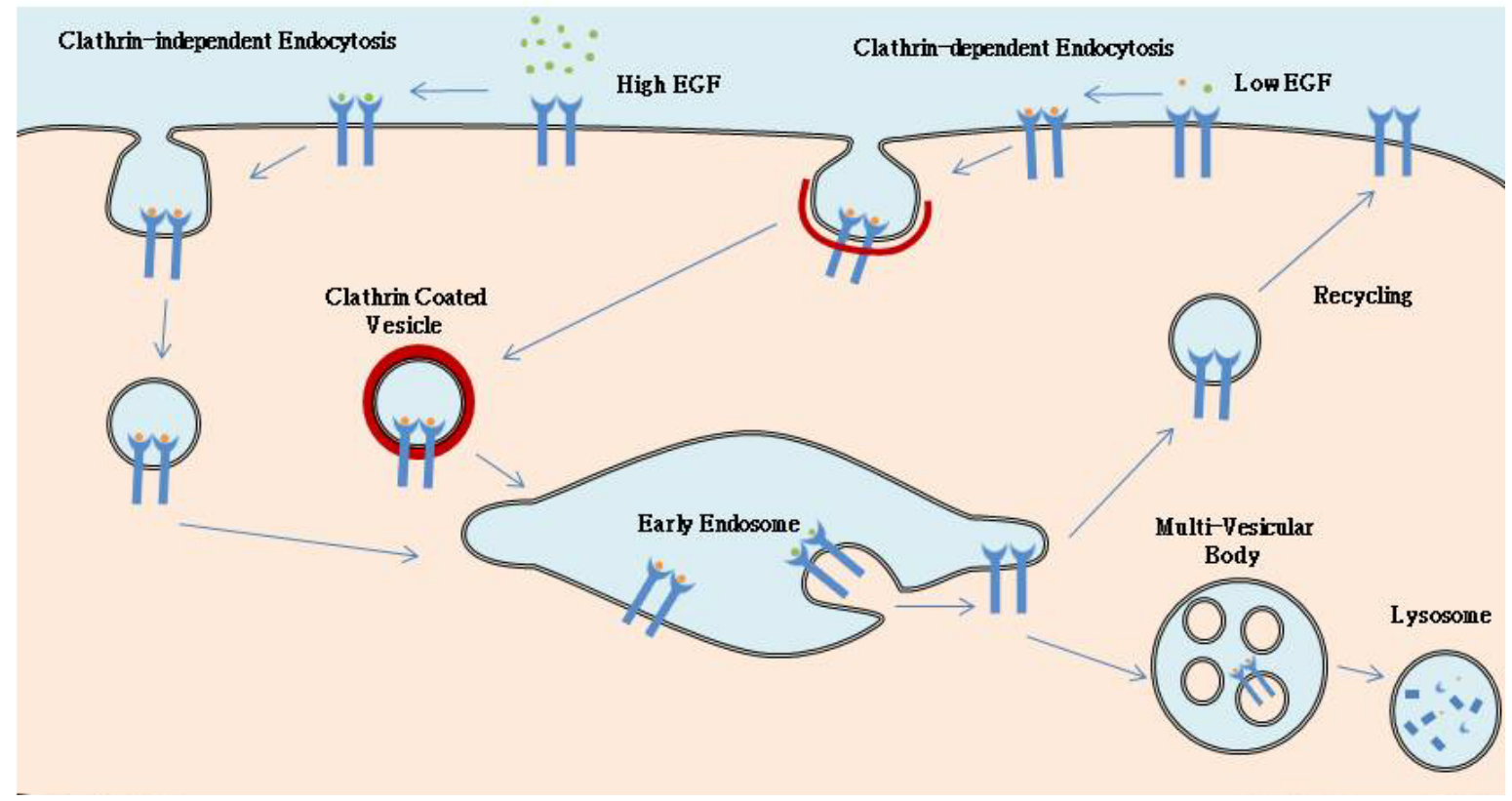

Figure 26. Endocytosis of EGF-Receptor. EGF-Receptor can be internalized through different pathways, depending on the concentration of ligand available to the cell. If the concentration of EGF is Low $(2 \mathrm{ng} / \mathrm{ml})$, the primary endocytic route will be through Clathrin Mediated Endocytosis (CME). However, if the concentration of EGF is high $(>100 \mathrm{ng} / \mathrm{ml})$, the receptor will be internalized primarily via various clathrin independent (CI) pathways, however some could still get in via CME. Internalized vesicle containing EGFR will then fuse with the early endosome prior to either being recycled to the cell surface or degraded in the lysosome. 

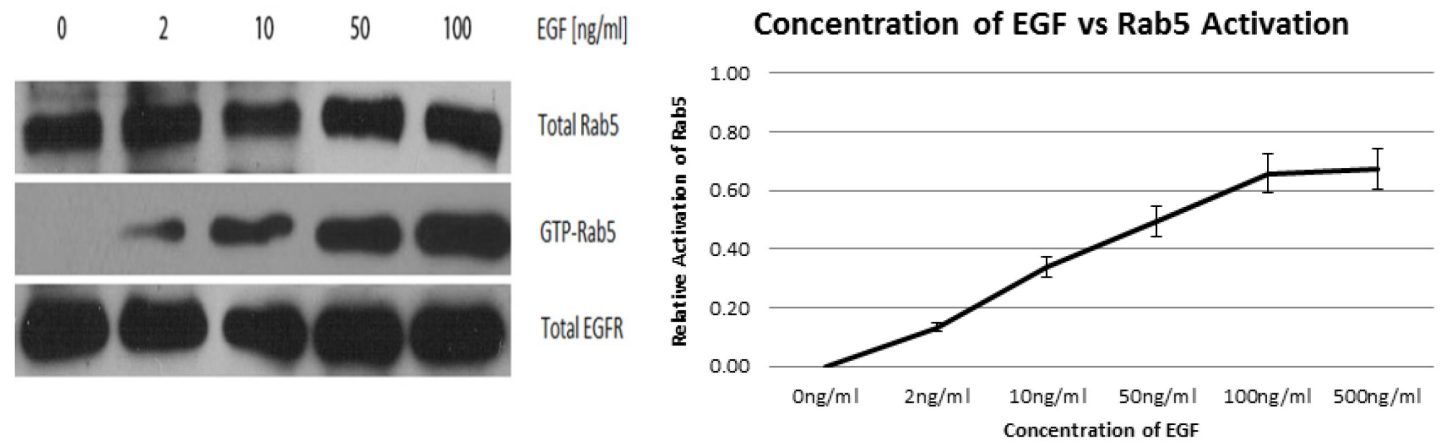

Figure 27. Effect of EGF on activation of Rab5. NR6-EGFR cells were transfected with Rab5, incubated with varying amounts of EGF $(0,2,10,50$ and $100 \mathrm{ng} / \mathrm{ml})$ at $4^{\circ} \mathrm{C}$ for $60 \mathrm{~min}$, washed with $\mathrm{PBS}$ and incubated for 5 minutes at $37^{\circ} \mathrm{C}$. They were subsequently washed with PBS, lysed and bound to glutathione beads in the presence of GST alone or GST-EEA1 at $4^{\circ} \mathrm{C}$ for 60 minutes. After incubation, beads were again washed with PBS and the presence of activated Rab5 was analyzed by western blot. Relative levels of these proteins were determined by densitometry. Data represents the mean $\pm \mathrm{SD}$ of three independent experiments. 
A
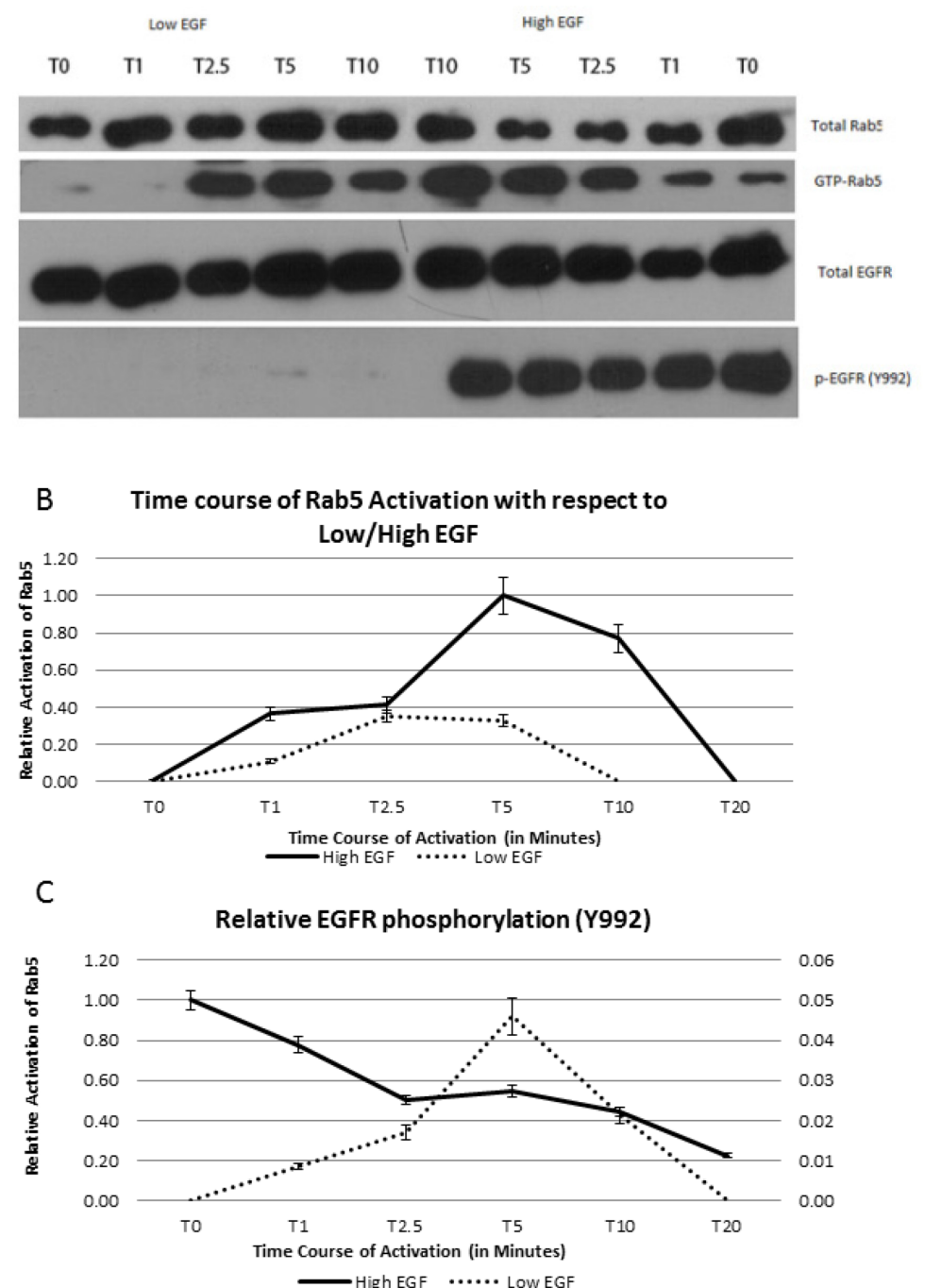

Figure 28. Temporal activation of Rab5 based on varying amounts of EGF. NR6EGFR cells were transfected with GFP-Rab5a, incubated with either $2 \mathrm{ng} / \mathrm{ml}$ or $100 \mathrm{ng} / \mathrm{ml}$ of EGF at $4^{\circ} \mathrm{C}$ for 60 minutes, washed with PBS and incubated for $0,1,2.5,5$ or 10 min at $37^{\circ} \mathrm{C}$. They were subsequently washed with PBS, lysed and bound to glutathione beads in the presence of GST alone or GST-EEA1 at $4^{\circ} \mathrm{C}$ for $60 \mathrm{~min}$. After incubation, beads were again washed with PBS and the presence of activated Rab5 was analyzed by western blot. The total cell lysate was also used to assay relative amounts of phosphorylated EGFR in each condition. Relative levels of these proteins were determined by densitometry. High EGF is based on the primary Y-axis and Low EGF corresponds to the secondary $\mathrm{Y}$-axis. Data represents the mean $\pm \mathrm{SD}$ of three independent experiments. 


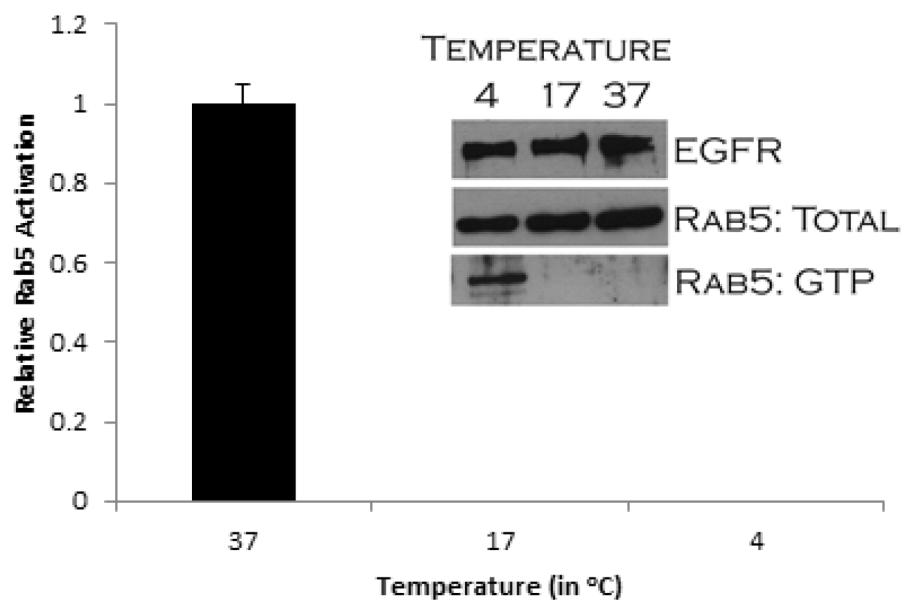

Figure 29. Effect of Temperature on Activation of Rab5. NR6-E cells were transfected with Rab5, incubated with $100 \mathrm{ng} / \mathrm{ml}$ of EGF at $4^{\circ} \mathrm{C}$ for $60 \mathrm{~min}$, washed with PBS and incubated for 5 minutes at $37^{\circ} \mathrm{C}$. They were subsequently washed with PBS, lysed and bound to glutathione beads in the presence of GST alone or GST-EEA1 at $4^{\circ} \mathrm{C}$ for 60 minutes. After incubation, beads were again washed with PBS and the presence of activated Rab5 was analyzed by western blot. Relative levels of these proteins were determined by densitometry. Data represents the mean $\pm \mathrm{SD}$ of three independent experiments. 
A Effect of Chlorpromazine on activation of Rab5
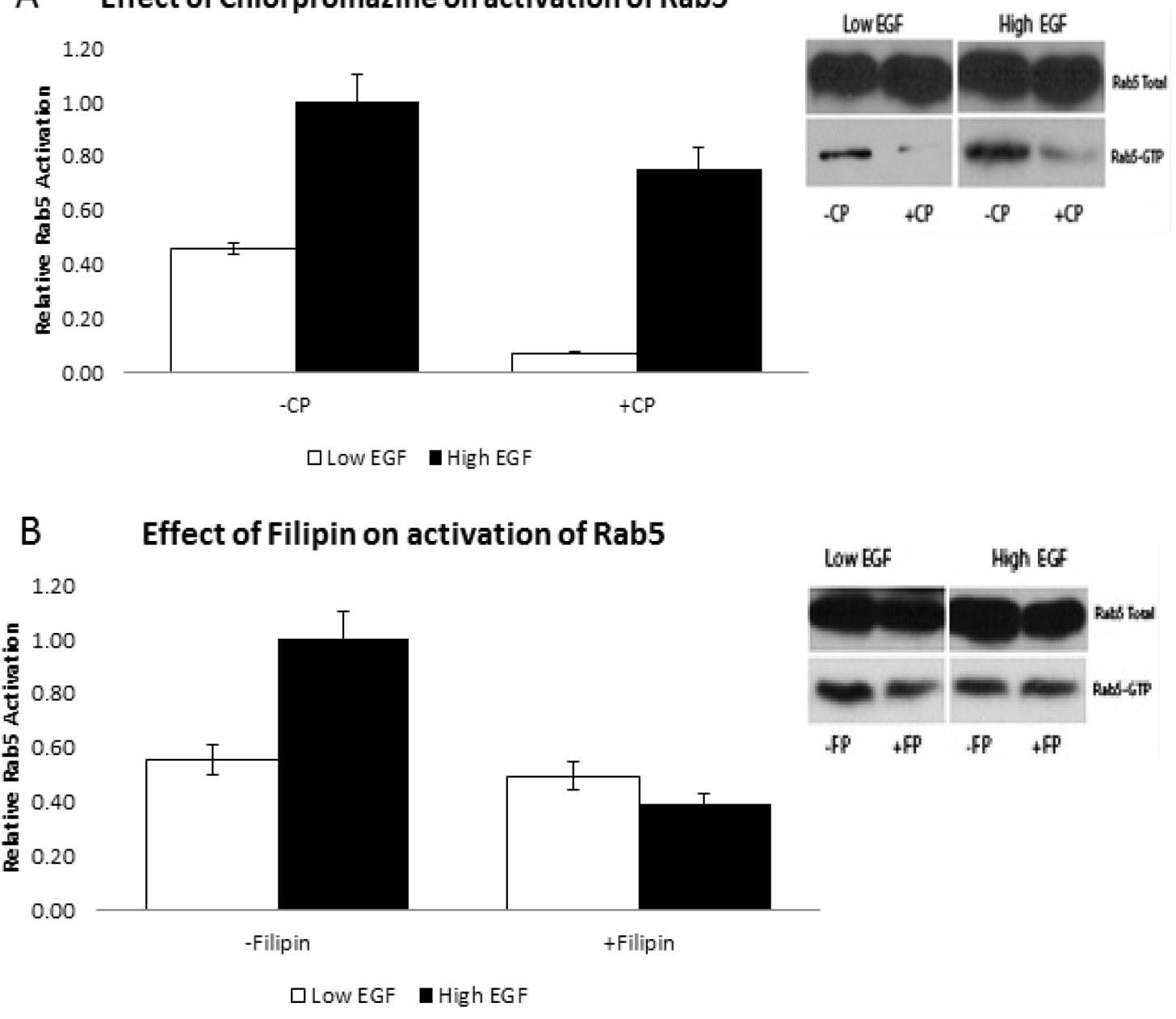

C

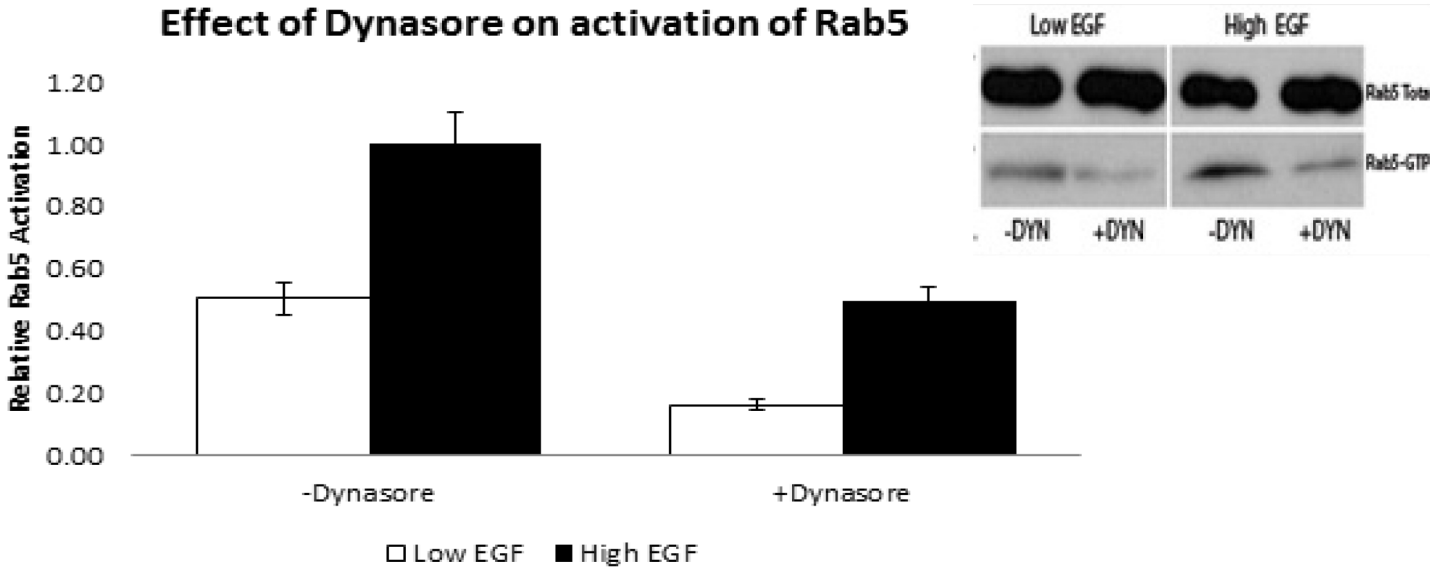




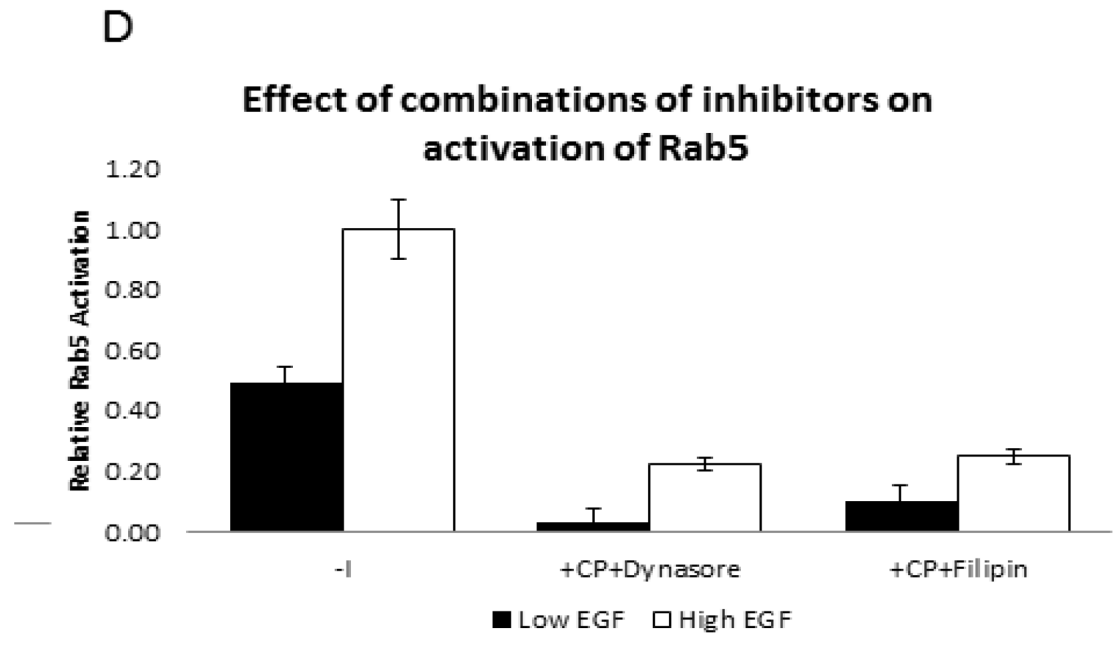

Figure 30. Effect of inhibitors of endocytosis on activation of Rab5. NR6-E cells were transfected with GFP-Rab5a, pretreated with either: a) Chlorpromazine $[20 \mu \mathrm{M}]$, b) Filipin $[5 \mu \mathrm{g} / \mathrm{ml}], \mathrm{c})$ Dynasore $[80 \mu \mathrm{M}]$ or d) combination of the three inhibitors for 30 minutes at $4^{\circ} \mathrm{C}$, incubated with $2 \mathrm{ng} / \mathrm{ml}$ or $100 \mathrm{ng} / \mathrm{ml}$ of EGF at $4^{\circ} \mathrm{C}$ for $60 \mathrm{~min}$, washed with PBS and incubated for $2.5 \mathrm{~min}$ at $37^{\circ} \mathrm{C}$. They were subsequently washed with PBS, lysed and bound to glutathione beads in the presence of GST alone or GST-EEA1 at $4{ }^{\circ} \mathrm{C}$ for $60 \mathrm{~min}$. After incubation, beads were again washed with PBS and the presence of activated Rab5 was analyzed by western blot. Relative levels of these proteins were determined by densitometry. Data represents the mean $\pm \mathrm{SD}$ of three independent experiments 

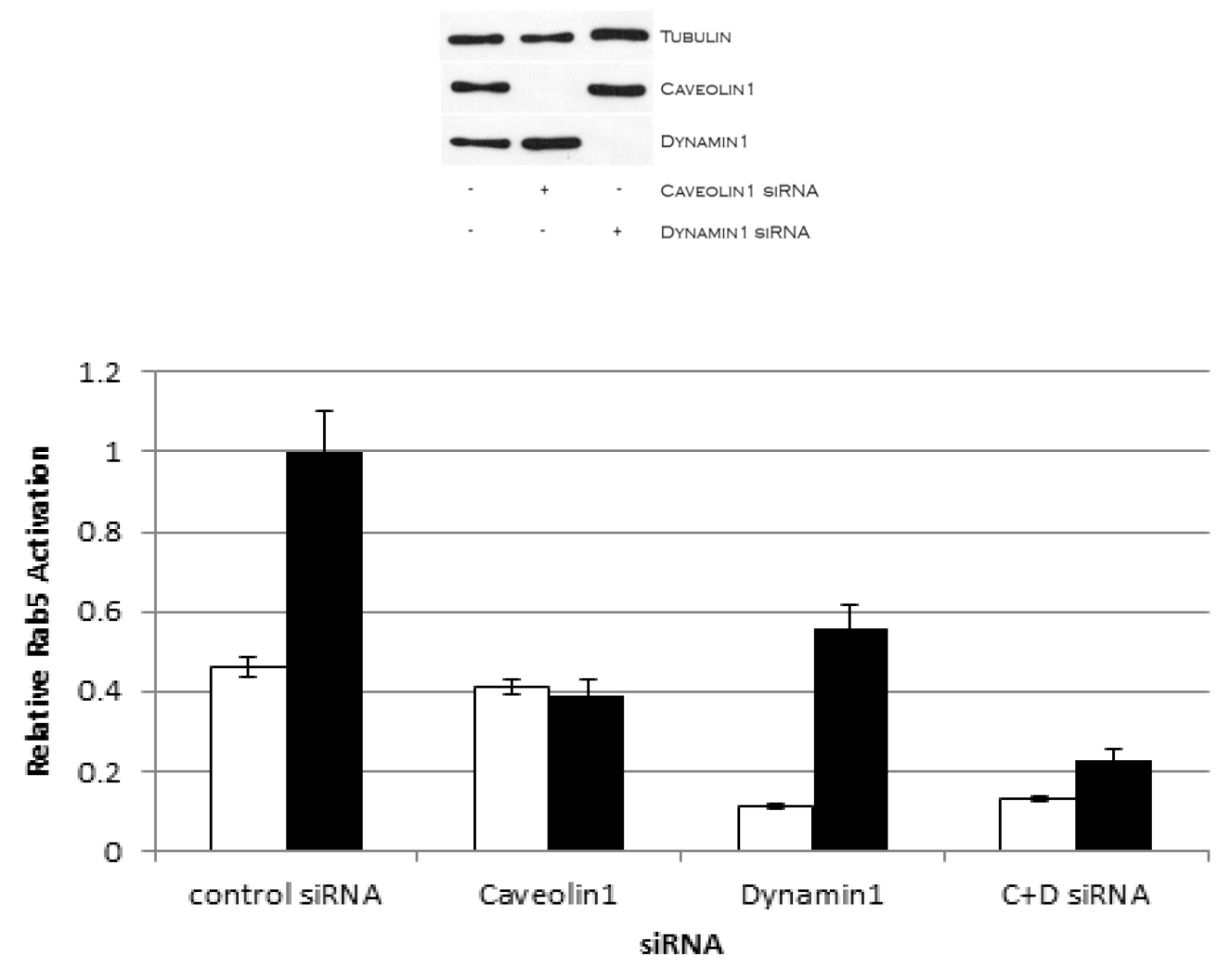

$\square$ Low EGF $\mathbf{n i g h}$ EGF

Figure 31. Effect of siRNA knockdown of Caveolin and Dynamin on activation of Rab5. NR6-E cells were transfected with GFP-Rab5a, and then with siRNA against Caveolin 1, Dynamin 2 (or a combination) and incubated with $2 \mathrm{ng} / \mathrm{ml}$ or $100 \mathrm{ng} / \mathrm{ml}$ of EGF at $4^{\circ} \mathrm{C}$ for $60 \mathrm{~min}$, washed with PBS and incubated for $2.5 \mathrm{~min}$ at $37^{\circ} \mathrm{C}$. They were subsequently washed with PBS, lysed and bound to glutathione beads in the presence of GST alone or GST-EEA1 at $4^{\circ} \mathrm{C}$ for $60 \mathrm{~min}$. After incubation, beads were again washed with PBS and the presence of activated Rab5 was analyzed by western blot. Relative levels of these proteins were determined by densitometry. Data represents the mean $\pm \mathrm{SD}$ of three independent experiments. 


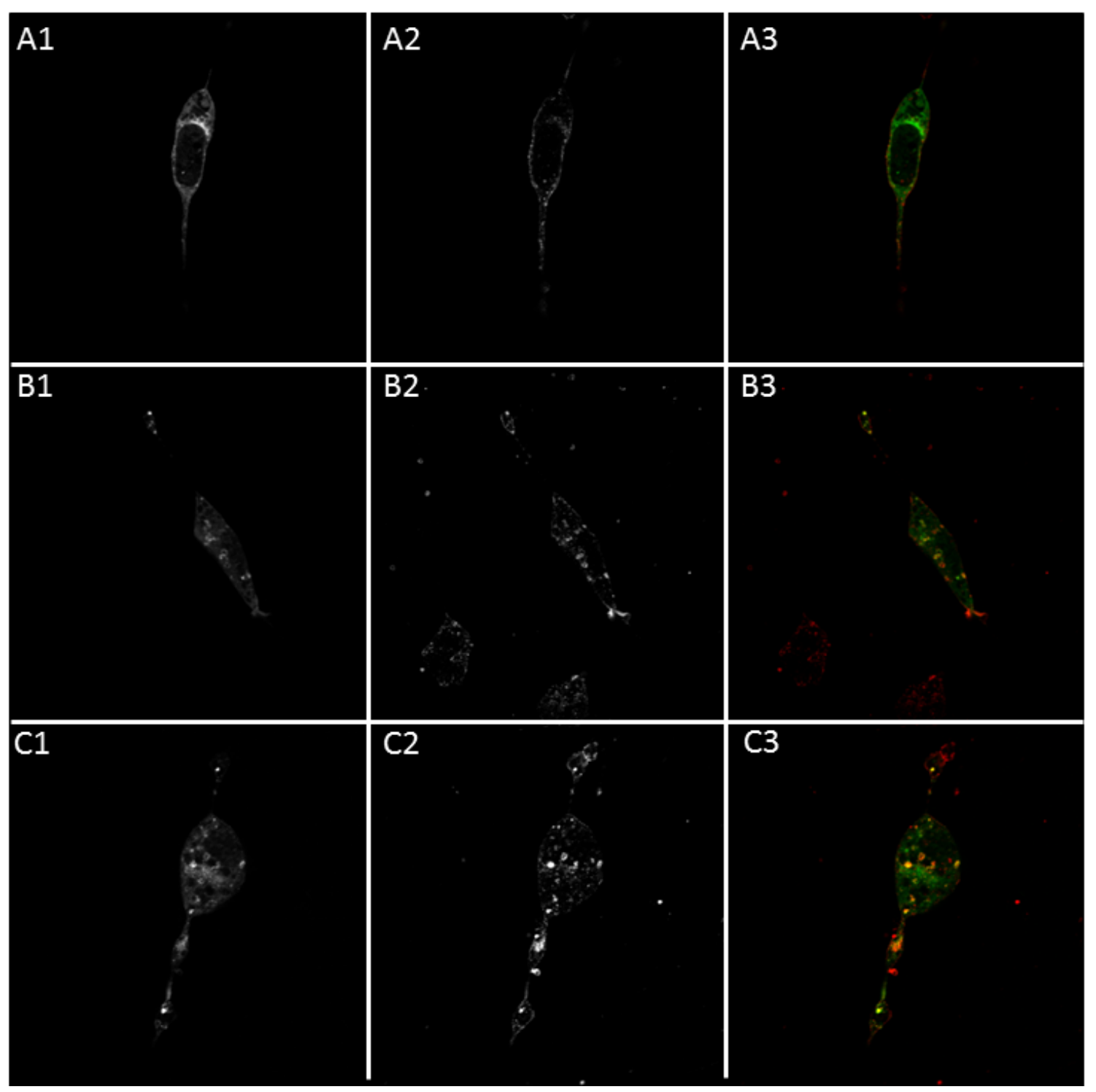

Figure 32: Effect of inhibitors of endocytosis on the formation of enlarged Rab5posotive endosomes. NR6-E cells were transfected with GFP-Rab5a, pretreated with either: no inhibitor $(A-C)$, Chlorpromazine $[20 \mu \mathrm{M}](\mathrm{D}, \mathrm{E})$, Dynasore $(\mathrm{F}, \mathrm{G})[80 \mu \mathrm{M}]$, Filipin $(\mathrm{H}, \mathrm{I})[5 \mu \mathrm{g} / \mathrm{ml}]$, Chlorpromazine \& Dynasore $(\mathrm{J}, \mathrm{K})$ or Chlorpromazine and Filipin $(\mathrm{L}, \mathrm{M})$ for $30 \mathrm{~min}$ at $4^{\circ} \mathrm{C}$, incubated with $0(\mathrm{~A}), 2 \mathrm{ng} / \mathrm{ml}(\mathrm{B}, \mathrm{D}, \mathrm{F}, \mathrm{H}, \mathrm{J}, \mathrm{L})$ or $100 \mathrm{ng} / \mathrm{ml}(\mathrm{C}$, $\mathrm{E}, \mathrm{G}, \mathrm{I}, \mathrm{K}, \mathrm{M}$ ) of EGF at $4^{\circ} \mathrm{C}$ for $60 \mathrm{~min}$, washed with PBS and incubated for $2.5 \mathrm{~min}$ at $37^{\circ} \mathrm{C}$. They were subsequently washed with PBS, fixed with $4 \%$ paraformaldehyde for 20 min at room temperature, permeabilized with $0.1 \%$ Triton X-100 prior to incubation with the antibodies. Primary antibody used was rabbit anti EGF Receptor, while secondary antibody used was Alexa594 labeled goat anti-rabbit IgG antibody. GFP-Rab5a (1); immunofluorescence against EGFR (2); merge (3). 


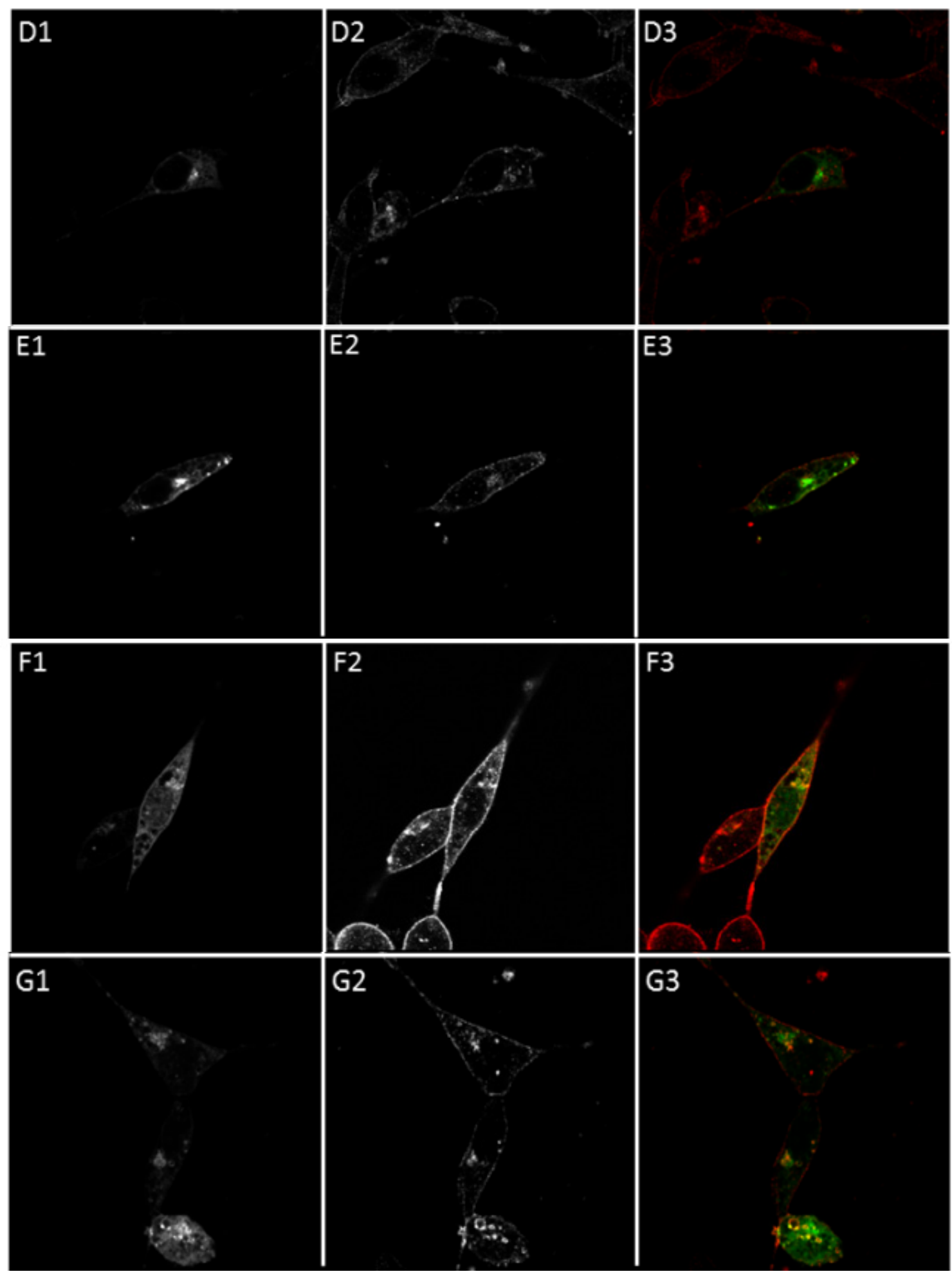

Figure 32 Continued 


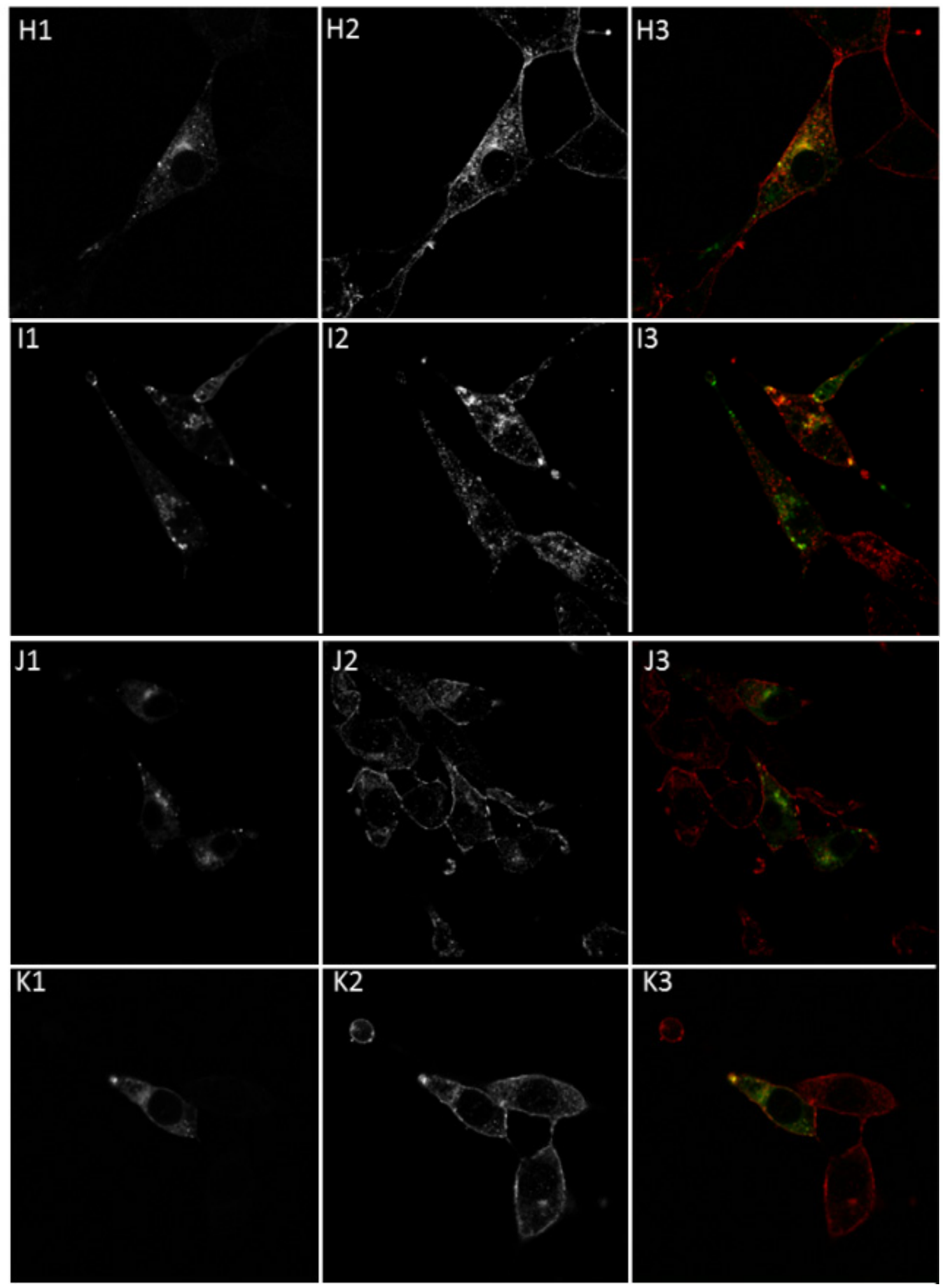

Figure 32 Continued 


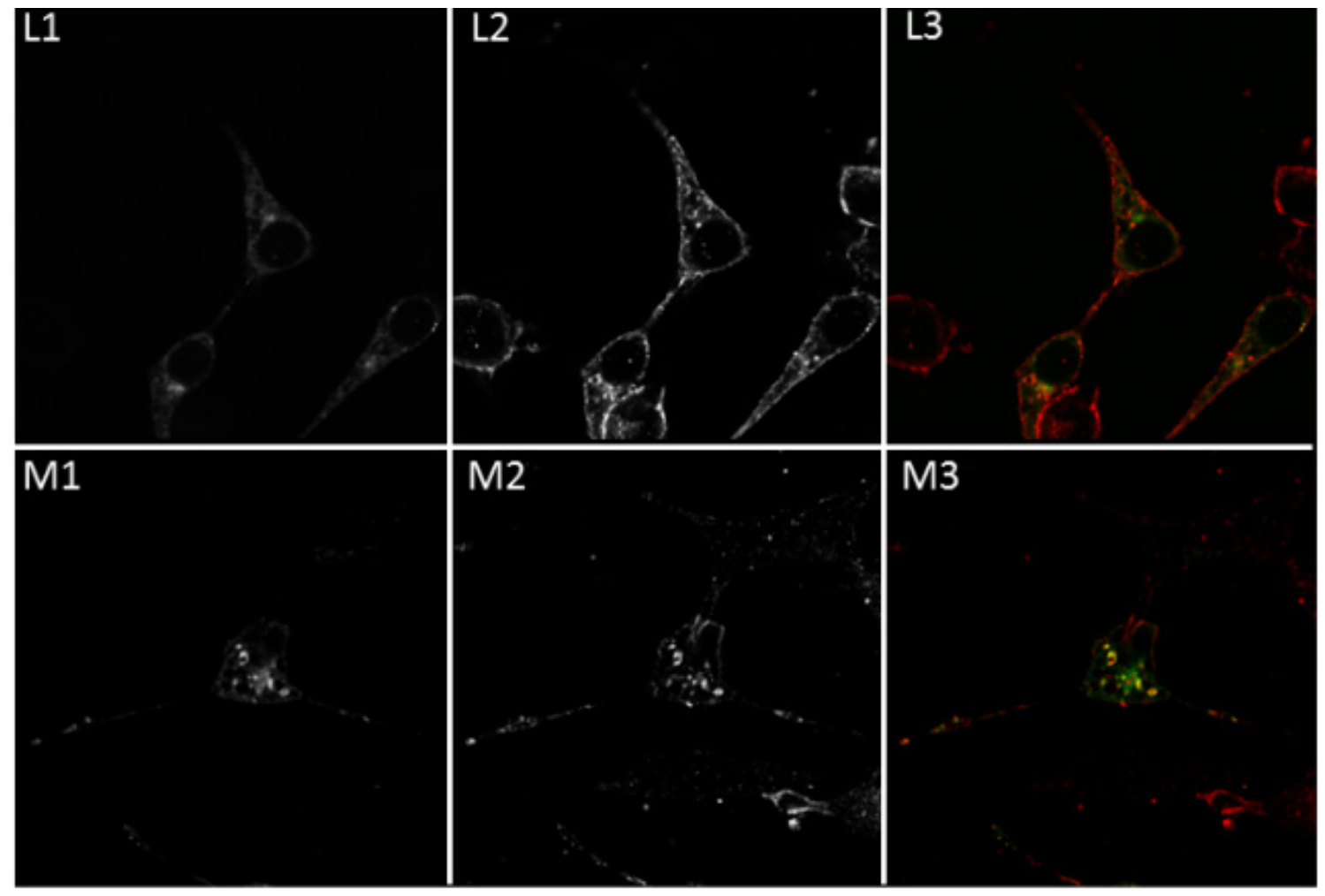

Figure 32 Continued 
Rin1

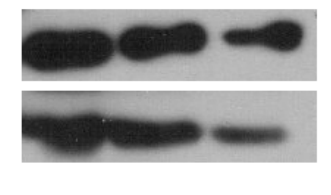

- +

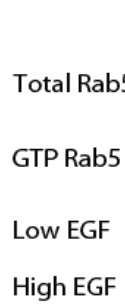

Rabex 5
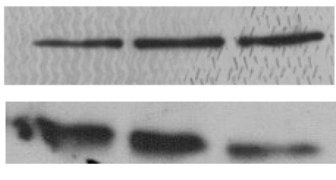
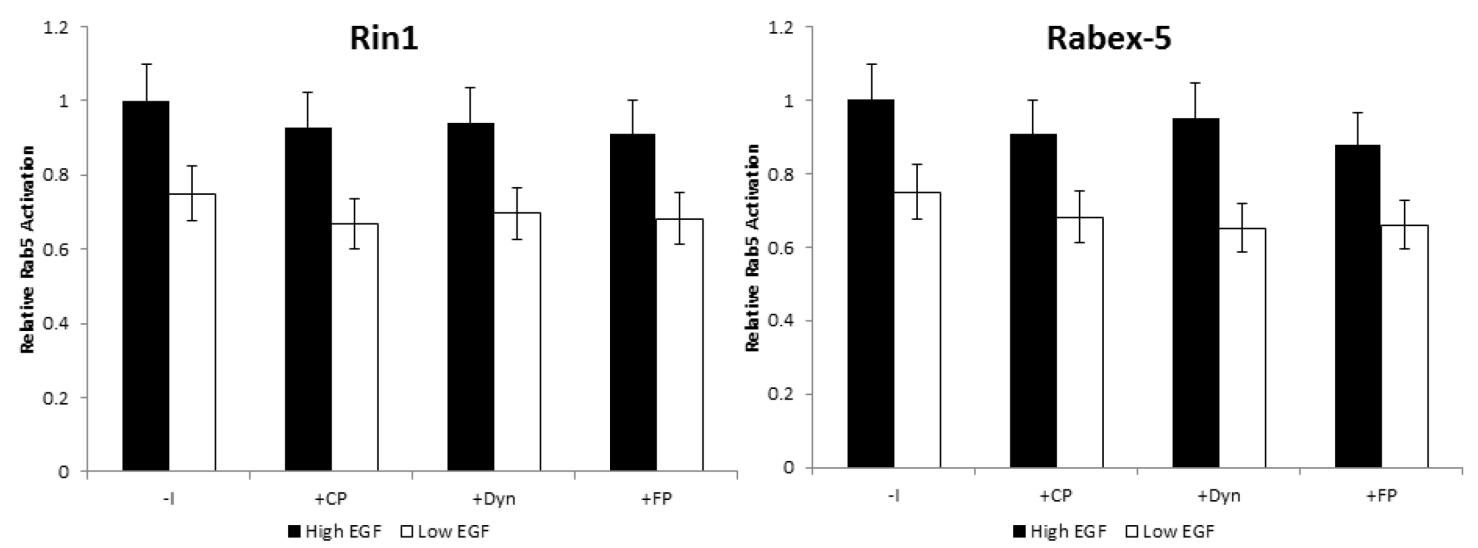

Figure 33. Effect of overexpression of Rabex-5 or Rin 1 on activation of Rab5. a) NR6-E cells expressing either Rabex-5: FL or Rin1: FL were transfected with Rab5a, incubated with $2 \mathrm{ng} / \mathrm{ml}$ or $100 \mathrm{ng} / \mathrm{ml}$ of EGF at $4^{\circ} \mathrm{C}$ for $60 \mathrm{~min}$, washed with PBS and incubated for $2.5 \mathrm{~min}$ at $37^{\circ} \mathrm{C}$. They were subsequently washed with PBS, lysed and bound to glutathione beads in the presence of GST alone or GST-EEA 1 at $4^{\circ} \mathrm{C}$ for 60 min. After incubation, beads were again washed with PBS and the presence of activated Rab5 was analyzed by western blot. b) NR6-E cells expressing either Rabex-5: FL or Rin1: FL were transfected with Rab5a, pretreated with either: Chlorpromazine $[20 \mu \mathrm{M}]$, Dynasore $[80 \mu \mathrm{M}]$ or Filipin $[1 \mathrm{mg} / \mathrm{ml}]$ for $30 \mathrm{~min}$ at $4^{\circ} \mathrm{C}$, incubated with $2 \mathrm{ng} / \mathrm{ml}$ or $100 \mathrm{ng} / \mathrm{ml}$ of EGF at $4^{\circ} \mathrm{C}$ for $60 \mathrm{~min}$, washed with PBS and incubated for $2.5 \mathrm{~min}$ at $37^{\circ} \mathrm{C}$. They were subsequently washed with PBS, lysed and bound to glutathione beads in the presence of GST alone or GST-EEA1 at $4^{\circ} \mathrm{C}$ for $60 \mathrm{~min}$. After incubation, beads were again washed with PBS and the presence of activated Rab5 was analyzed by western blot. Relative levels of these proteins were determined by densitometry. Data represents the mean $\pm \mathrm{SD}$ of three independent experiments. 
A

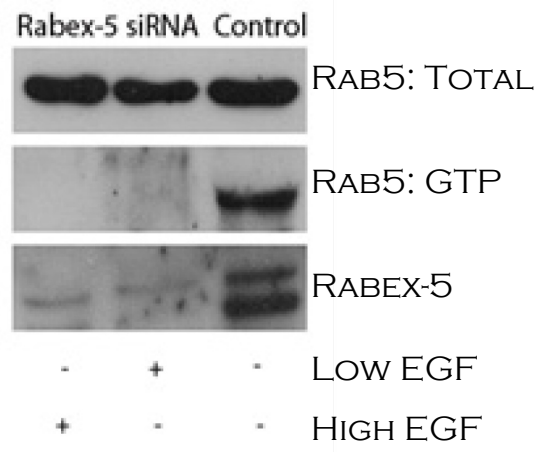

$\mathrm{B}$
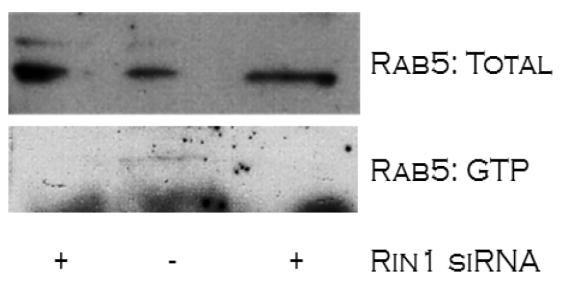

$-\quad+\quad+$ LOW EGF

$+\quad+\quad-$ HIGH EGF
$\mathrm{C}$

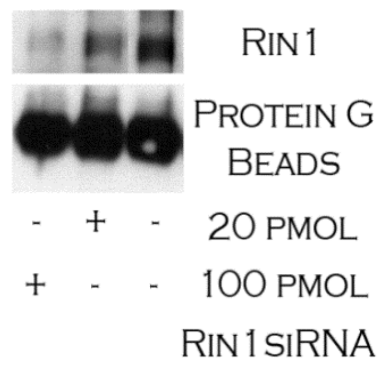

Figure 34. Effect of silencing of Rabex-5 or Rin1 on activation of Rab5. NR6-E cells were transfected with either A) Rabex-5 or B) Rin1 siRNA, incubated with $2 \mathrm{ng} / \mathrm{ml}$ or $100 \mathrm{ng} / \mathrm{ml}$ of EGF at $4^{\circ} \mathrm{C}$ for $60 \mathrm{~min}$, washed with PBS and incubated for $2.5 \mathrm{~min}$ at $37^{\circ} \mathrm{C}$. They were subsequently washed with PBS, lysed and bound to glutathione beads in the presence of GST alone or GST-EEA1 at $4^{\circ} \mathrm{C}$ for $60 \mathrm{~min}$. After incubation, beads were again washed with PBS and the presence of activated Rab5 was analyzed by western blot C) Since endogenous levels of Rin1 were not detectable, immunoprecipitation of total cell lysate using ProteinG beads and monoclonal Rin1 antibody was also performed to check for siRNA efficacy. 

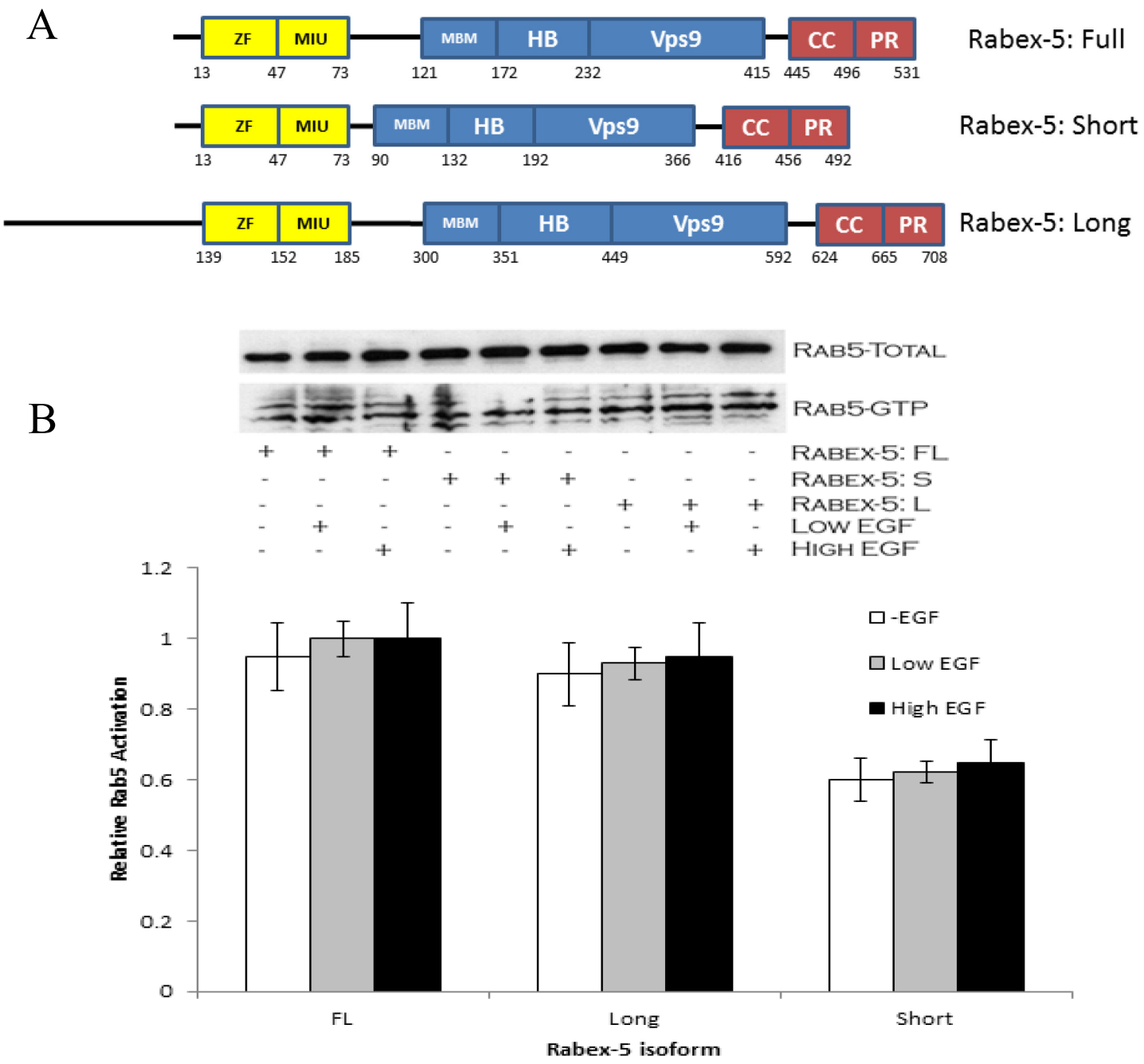

Figure 35. Effect of Rabex-5 splice variants on activation of Rab5. a) Primary structure of Rabex -5 splice variants. The Short and the Long splice variants contain extra 40 amino acids between the MIU and MBM domains in comparison to Rabex-5: FL, whereas Rabex-5 short is missing the terminal 124 amino acids in comparison to Rabex5: FL and Rabex-5: Long. b) Rabex-5 splice variants differentially activate Rab5. NR6-E cells expressing Rabex-5 in absence of EGF stimulation were lysed and bound to glutathione beads in the presence of GST alone or GST-EEA1 at $4^{\circ} \mathrm{C}$ for $60 \mathrm{~min}$. After incubation, beads were again washed with PBS and the presence of activated Rab5 was analyzed by western blot. Relative levels of these proteins were determined by densitometry. Data represents the mean \pm SD of three independent experiments. 


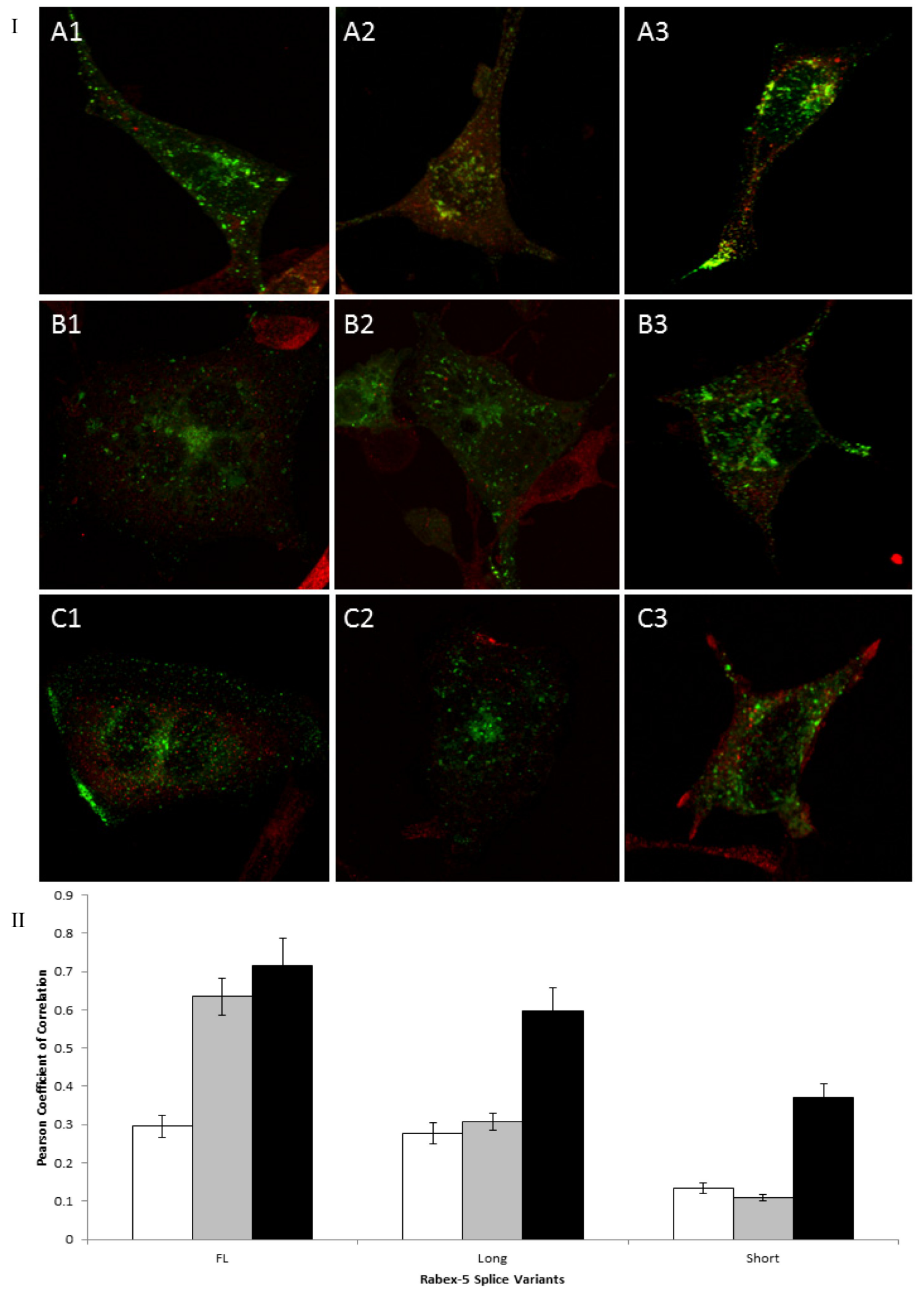

口-EGF $\quad$ low EGF high EGF 
Figure 36. Effect of Rabex-5 splice variants on formation of Rab5 positive endosomes. I) Confocal Images showing co-localization of Rabex-5 and Rab5. NR6E cell lines expressing either Rabex-5: FL, Rabex-5: Long or Rabex-5: Short splice variant were transfected with GFP-Rab5wt, after which EGF uptake was carried out using 0, 2 or $100 \mathrm{ng} / \mathrm{ml}$ of EGF for $2.5 \mathrm{~min}$ at $37^{\circ} \mathrm{C}$. Cells were fixed with paraformaldehyde and immunofluorescence was performed using anti Rabex-5 rabbit polyclonal primary and goat anti rabbit Alexa594 secondary antibody. A-Rabex-5: Full Length; B-Rabex5: Long splice variant; C-Rabex5: Short splice variant; 1- No EGF; 2-Low EGF; 3-High EGF. II) Pearson correlation coefficients showing co-localization of Rab5 with Rabex-5 from each condition. 
A

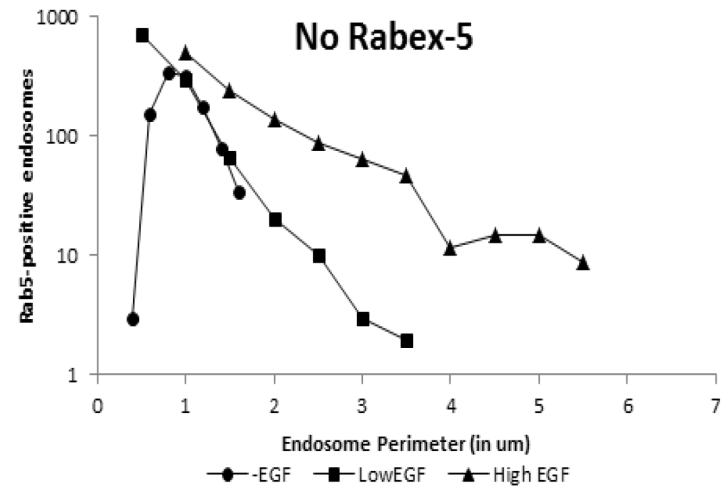

C

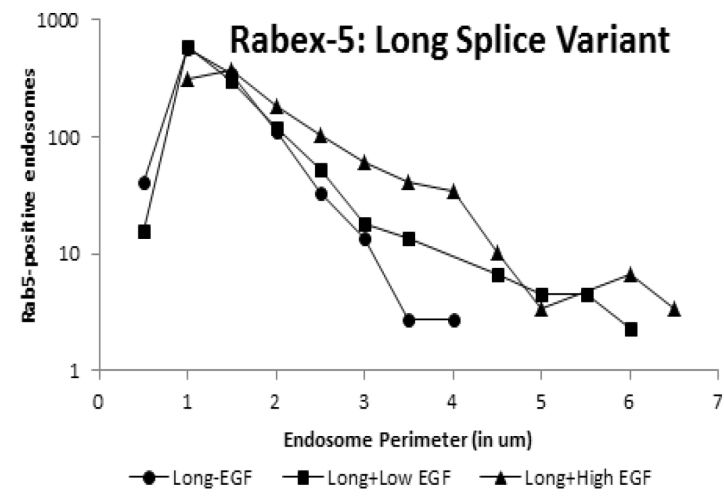

B

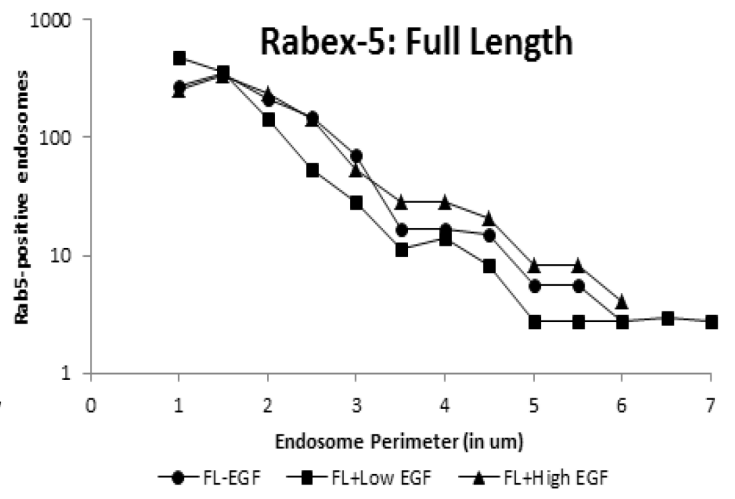

D

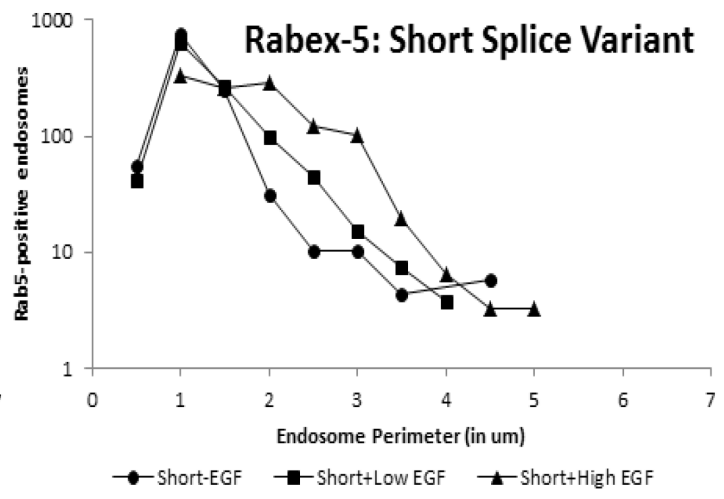

Figure 37. Effect of Rabex-5 splice variants on perimeter of Rab5-positive endosomes. Perimeters of Rab5-positive endosomes from at least 200 cells were measured using the NIH Image $J$ software from immunofluorescence images acquired using a Leica TCS SP2 confocal microscope in NR6 cells for each experimental condition. Histograms of Rab5-positive endosomes vs. the endosome size were determined in NR6-E cells expressing Rab5 wild type (WT) containing either Rabex-5: FL, Rabex-5: Long or Rabex-5: Short splice variants in the absence or in the presence of either 2 or $100 \mathrm{ng} / \mathrm{ml} \mathrm{EGF.}$ 


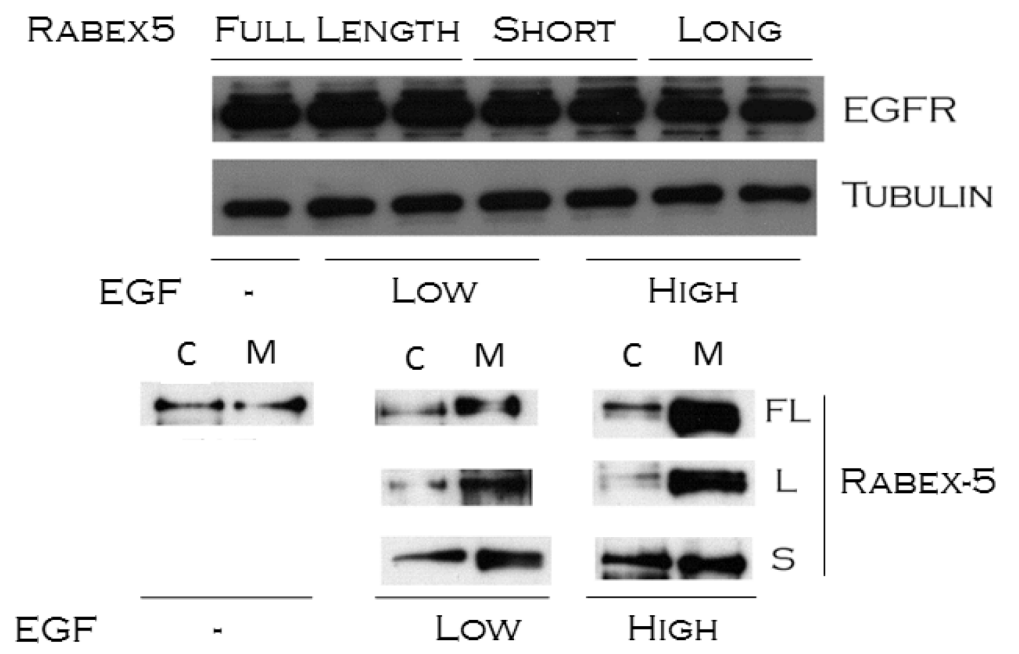

Figure 38. Subcellular Distribution of Rabex-5 splice variants. NR6-E cells expressing either Rabex-5: FL (FL), Rabex-5: Long (L) or Rabex-5: Short (S) splice variants were incubated with 0,2 or $100 \mathrm{ng} / \mathrm{ml}$ EGF as described in Materials and Methods. After treatment, cells were washed with ice cold PBS and incubated at $37^{\circ} \mathrm{C}$ for 5 minutes. Cells were then washed again using ice-cold PBS, homogenized and membrane fractions were prepared as described in Materials and Methods. Membrane $[\mathrm{M}]$ and cytosol [C] fractions were treated with sample buffer and proteins were subject to SDS-PAGE, blotted to a nitrocellulose membrane, and antibodies specific to Rabex-5, phospho(p)-EGF-receptor, total(t)-EGF-receptor, and tubulin were used to visualize these proteins by Western blot analysis. Relative levels of these proteins were determined by densitometry. Data represents the mean \pm SD of four independent experiments. 


\subsection{References}

Barbieri M.A., Roberts R.L., Gumusboga A., Highfield H., Alvarez-Dominguez C., Wells A., Stahl P.D. 2000. Epidermal growth factor and membrane trafficking. EGF receptor activation of endocytosis requires Rab5a. J. Cell Biol. 151: 539-550.

Barbieri M.A., Fernandez-Pol S., Hunker C., Horazdovsky B.H., Stahl P.D. 2004. Role of Rab5 in EGF receptor-mediated signal transduction. Eur. J. Cell Biol. 83: 305-314.

Barral D.C, Ramalho J.S., Anders R, Hume A.N., Knapton H.J., Tolmachova T., Collinson L.M., Goulding D., Authi K.S., Seabra M.C. 2002. Functional redundancy of Rab27 proteins and the pathogenesis of Griscelli syndrome. J Clin Invest. 110(2): 247257.

Carpenter G. \& Cohen S. 1976. I-Labeled human epidermal growth factor: binding internalization, and degradation in human fibroblasts. J. Cell Biol. 71: 151-171.

Chadda R. 2007. Cholesterol-sensitive CDC42 activation regulates actin polymerization for endocytosis via the GEEC pathway. Traffic. 8: 702-717.

Damke H., Baba T., van der Bliek A.M., Schmid S.L. 1995. Clathrin-independent pinocytosis is induced in cells overexpressing a temperature sensitive mutant of dynamin. J. Cell Biol. 131: 69-80.

Dinneen J.L. and Ceresa B.P. 2004. Expression of dominant negative rab5 in HeLa cells regulates endocytic trafficking distal from the plasma membraneExp. Cell Res. 294: 509522.

Doherty G.J. \& McMahon H.T. 2009. Mechanisms of Endocytosis. Annu. Rev. Biochem. 78: 857-902.

D'Souza-Schorey C. \& Chavrier P. 2006. ARF proteins: roles in membrane traffic and beyond. Nature Rev. Mol. Cell Biol. 7: 347-358.

Frick M., Bright N.A., Riento K., Bray A., Merrified C., Nichols B.J. 2007. Coassembly of flotillins induces formation of membrane microdomains, membrane curvature, and vesicle budding. Curr. Biol. 17:1151-56.

Fukuda M. 2008. Regulation of secretory vesicle traffic by Rab small GTPases. Cell Mol. Life Sci. 65(18): 2801-2813.

Giocondi M.C., Mamdouh Z., Le Grimellec C. 1995. Benzyl alcohol differentially affects fluid phase endocytosis and exocytosis in renal epithelial cells. Biochim Biophys Acta 1234: 197-202.

Grant, B.D. \& Donaldson, J.G. 2009. Pathways and mechanisms of endocytic recycling. Nature Rev. Mol. Cell Biol. 10: 597-608. 
Hopkins, C.R., Miller, K. \& Beardmore, J.M. 1985. Receptor-mediated endocytosis of transferrin and epidermal growth factor receptors: a comparison of constitutive and ligand-induced uptake. J. Cell Sci. 3: 173-186.

Howes, M.T., Kirkham M., Riches J., Cortese K., Walser P.J., Simpson F., Hill M.M., Jones A., Lundmark R., Lindsay M.R., Hernandez-Deviez D.J., Hadzic G., McCluskey A., Bashir R., Liu L., Pilch P., McMahon H., Robinson P.J., Hancock J.F., Mayor S., Parton R.G. 2010. Clathrin-independent carriers form a high capacity endocytic sorting system at the leading edge of migrating cells. J. Cell Biol. 4: 675-691.

Ivanov A.I. 2008. Exocytosis and endocytosis. Methods in Molecular Biology 440.

Jozic I., Saliba S.C., Barbieri A.M. 2012. Effect of EGF-receptor tyrosine kinase inhibitor on Rab5 function during endocytosis. Arch. Biochem. Biophys. 525(1): 16-24.

Kirkham M. \& Parton R.G. 2005. Clathrin-independent endocytosis: new insights into caveolae and noncaveolar lipid raft carriers. Biochim. Biophys. Acta. 1746: 349-363.

Kitajima Y., Sekiya T., Nozawa Y. 1976. Freeze fracture ultrastructural alterations induced by filipin, pimaricin, nystatin amphotericin $\mathrm{B}$ in plasma membranes of Epidermophyton, Saccharomyces and red complex-induced membrane lesions. Biochim. Biosphys. Acta. 455: 452-465.

Kumari S. \& Mayor S. 2008. ARF1 is directly involved in dynamin-independent endocytosis. Nat. Cell Biol. 10: 30-41.

Lamaze C., Dujeancourt A., Baba T., Lo C.G., Benmerah A., Dautry-Varsat A. 2001. Interleukin 2 receptors and detergent-resistant membrane domains define a clathrinindependent endocytic pathway. Mol. Cell. 7:661-61.

Lenhard J.M., Mayorga L. \& Stahl P.D. 1992. Characterization of Endosome-Endosome Fusion in a Cell-free system using Dictyostelium discoideum. 1992. J. of Biol. Chem. 267(3): 1896-1903.

Macia E., Ehrlich M., Massol R., Boucrot E., Brunner C. Kirchhausen T. 2006. Dynasore, a cell permeable inhibitor of dynamin. Dev. Cell. 10. 839-850.

Mayor S. \& Pagano R.E. 2007. Pathways of clathrin-independent endocytosis. Nature Rev. Mol. Cell Biol. 8: 603-12.

McMahon H.T. \& Boucrot E. 2011. Molecular mechanism and physiological functions of clathrin-mediated endocytosis. Nat. Rev. Mol. Cell Biol. 12: 517-533.

Ogiso T., Iwaki M., Mori K. 1981. Fluidity of human erythrocyte membrane and effect of chlorpromazine on fluidity and phase separation of membrane. Biochim Biophys Acta 649: $322-335$. 
Orlandi, P.A. and Fishman, P.H. 1998. Filipin-dependent inhibition of cholera toxin: evidence for toxin internalization and activation through caveolae-like domains. J. Cell Biol. 141: 905-915.

Overholtzer M., Mailleux A.A., Mouneimne G., Normand G., Schnitt S.J., King R.W., Brugge J.S. 2007. A nonapoptotic cell death process, entosis, that occurs by cell-in-cell invasion. Cell. 131:966-79.

Qualmann B. \& Mellor H. 2003. Regulation of endocytic traffic by Rho GTPases. Biochem. J. 371:233-241.

Parton R.G \& Simons K. 2007. The multiple faces of caveolae. Nature Rev. Mol. Cell Biol. 8: 185-194.

Rejman, J., Bragonzi, A., Conesse M. 2005. Role of Clathrin and Caveolin Mediated endocytosis in gene transfer mediated by Lipo and polyplexes. Molecular Therapy. 12: 468-474.

Ros-Baro A., Lopez-Iglesias C., Peiro S. 2001. Lipid rafts are required for GLUT4 internalization in adipose cells. PNAS USA. 98:12050-12055.

Roth TF, Porter KR. 1964. Yolk protein uptake in the oocyte of the mosquito Aedes aegypti L. J. Cell Biol. 20:313-32.

Rothberg KG, Heuser JE, Donzell WC, Ying YS, Glenney JR, Anderson RG. 1992. Caveolin, a protein component of caveolae membrane coats. Cell 68:673-82.

Sabharanjak S., Sharma P., Parton R.G., Mayor S. 2002. GPI-anchored proteins are delivered to recycling endosomes via a distinct CDC42-regulated, clathrin independent pinocytic pathway. Dev. Cell. 2: 411-423.

Schnitzer, J. E., Oh, P., Pinney, E. and Allard, J. 1994. Filipin-sensitive caveolaemediated transport in endothelium: reduced transcytosis, scavenger endocytosis, and capillary permeability of select macromolecules. J. Cell Biol. 127: 1217-1232.

Shao Y, Akmentin W,Toledo-Aral JJ, Rosenbaum J,Valdez G, et al. 2002. Pincher, a pinocytic chaperone for nerve growth factor/TrkA signaling endosomes. J. Cell Biol. 157: 679-91.

Simons K. \& Ikonen E. 1997. Functional rafts in cell membranes. Nature. 387: 569-572.

Sorkin A. \& Goh L.K. Endocytosis and intracellular trafficking of ErbBs. 2008. Exp. Cell Res. 314(17): 3093-3106.

Tsuboi T. \& Fukuda M. 2006. Rab3A and Rab27A cooperatively regulate the docking step of dense-core vesicle exocytosis in PC12 cells. J. Cell Sci. 119: 2196-2203. 
Walenga R.W., Opas E.E., Feinstein M.B. 1981. Differential effect ofcalmodulin antagonists on phospholipases $\mathrm{A} 2$ and $\mathrm{C}$ in thrombin-stimulated platelets. J. Biol. Chem. 256: $12523-12528$.

Wang, L.H., Rothberg, K.G. and Anderson, R.G. 1993. Mis-assembly of clathrin lattices on endosomes reveals a regulatory switch for coated pit formation. J. Cell Biol. 123: $1107-1117$.

Wells A. 1999. EGF Receptor. The Inter. J. of Biochem. \& Cell Biol. 31:637-643.

Zeigerer A., Gilleron J., Bogorad R.L., Marsico G., Nonaka H., Seifert S., Epstein-Barash H., Kuchimanchi S., Peng C.G., Ruda V.M., Del Conte-Zerial P., Hengsler J.G., Kalaidzidis Y., Koteliansky V., Zerial M. 2012. Rab5 is necessary for the biogenesis of endolysosomal system in vivo. Nature. 485: 465-470.

Zerial M. \& McBride H. 2001. Rab proteins as membrane organizers. Nature Rev. Mol. Cell Biol. 2: 107-117. 


\section{CHAPTER 4}

THE EFFECT OF RABEX-5 ON SEVERAL BIOLOGICAL PROCESSES 


\subsection{Abstract}

Alarming increases in global incidence of obesity-related health issues have also sparked immense interest into understanding the mechanisms behind the differentiation of pre-adipocytes into mature adipocytes, a process known as adipogenesis. Along the same lines, as humans are living longer and as the median age of the global world population is increasing, it should be of no surprise that the frequency of common neurodegenerative disorders (such as Parkinson's and Alzheimer's disease) has also been steadily rising. The Rab superfamily of small GTPases is a key regulator of numerous cellular trafficking processes including the endocytosis of GLUT4 and TrkA vesicles. Since Rab proteins play a critical role in vesicle trafficking, it may be fair to assume that they also may represent a site of integration for signals from insulin and TrkA receptor as well as signaling endosome biogenesis. Although extensive research has been centered on both adipogenesis as well as neurogenesis (and subsequently GLUT4 and TrkA trafficking), the specific role of Rab5, the master regulator of endosome biogenesis, in both of the aforementioned processes has yet to be determined. Here we investigated the role of Rabex-5, a Rab5 guanine nucleotide exchange factor that facilitates the exchange of GDP for GTP and thereby activates Rab5, in adipogenesis and neurogenesis. We found that Rabex-5 inhibits differentiation of both 3T3-L1 and PC12 cells not only by activating Rab5 and promoting degradation of the signaling endosome, but also by ubiquitinating Ras and inhibiting the Ras signaling cascade that is crucial for cellular differentiation. Further research may be needed to elucidate the full mechanism of Rabex-5 action in adipogenesis and neurogenesis as it may become a pharmaceutical target in battling obesity and various neurodegenerative disorders. 


\subsection{Introduction}

Since there has been a global rise in obesity-associated health issues [Kelly et al., 2008; Popkin et al., 2010; Flegal et al., 2010; Shao \& Chin 2011], the topic of adipogenesis has been getting an increasing amount of interest, which in turn has resulted in numerous advances in stem cell technology, cell isolation and identification, as well as trans-differentiation. One of the best studied and most established cell lines are the 3T3L1 pre-adipocytes, which are a continuous sub-strain of $3 \mathrm{~T} 3$ (Swiss albino) that were developed through clonal isolation. 3T3-L1 pre-adipocytes have no significant lipid content and resemble fibroblasts, but when cultured to differentiate, they undergo a preadipose to adipose like conversion while progressing from a rapidly dividing to a confluent and contact inhibited state that makes them are suitable for metabolic studies [Green \& Meuth, 1974].

Upon maturation, white adipocytes contain a single, massive lipid droplet that looks as if it comprises most of cell volume which thereby adding to the buoyance of the adipocyte. This makes it very difficult to culture and perform metabolic assays on, because the lipid-containing adipocytes tend to float and detach from their respective culture plates [Fernyhough et al., 2005]. Hence, most metabolic assays are not performed on fully mature adipocytes, but on those which are less buoyant and still able to adhere to culture plates. In general, most adipocytes possess a great capability to store immense volumes of lipid, which can subsequently be utilized by high priority tissues.

Variations in circulation of several hormone levels (such as insulin, thyroxine or growth hormone) as well as some glucocorticoids (such as dexamethasone) have been shown to often result in astonishing differences in the development of adipose tissue, 
because they all can stimulate adipocyte differentiation by triggering DNA replication, reentry of the cell cycle (a process known as mitotic clonal expansion), and a transcription factor cascade that ultimately leads to expression of various adipocyte genes [Mattsson \& Olsson, 2007; Hausman et al., 2009; Poulos et al., 2010; Tang \& Lane, 2012]. Lastly, elevation of cAMP (via activation of cAMP signaling pathways by supplementing the differentiation media with fetal calf serum) has also been shown to have a significant impact on induction of differentiation in 3T3-L1 pre-adipocytes [Student et al., 1980; MacDougald \& Lane, 1995; Tang \& Lane, 2012].

In a regular cycle of adipocyte differentiation, shortly (less than 5 minutes) after the induction of differentiation by a media cocktail containing (insulin or IGF-1, 3-isobutyl1-methylxanthine (more commonly referred to as IBMX) and dexamethasone), phosphorylation of CREB (cAMP response element binding protein) causes activation and expression of transcription factor $\mathrm{C} / \mathrm{EBP} \beta$ (CCAAT/enhancer-binding protein beta) causing growth arrest [Zhang et al., 2004]. Next, MAPK (Mitogen Activated Protein Kinase) and then GSK3 $\beta$ (Glycogen synthase kinase 3 beta) phosphorylate C/EBP $\beta$ on Thr 188 or Thr 179 and Ser 187 [Tang et al., 2005] respectively, which thereby acquires DNA-binding activity and the pre-adipocytes synchronously re-enter the cell cycle. MAPK phosphorylation has been shown to occur within 4 hours of induction and is required for mitotic clonal expansion, C/EBP $\beta$ DNA binding activity and terminal differentiation while dual phosphorylation of GSK3 $\beta$, which occurs between 12-16 hours of induction, is what actually leads to acquisition of DNA-binding activity. At about 1620 hours after induction, pre-adipocytes synchronously reenter the cell cycle and undergo several rounds of mitosis, referred to as mitotic clonal expansion. As a failsafe 
mechanism to prevent the acquisition of DNA-binding activity of $\mathrm{C} / \mathrm{EBP} \beta$ until the preadipocytes have entered the mitotic clonal expansion, CHOP10 (C/EBP homologous protein 10) forms a heterodimer with $\mathrm{C} / \mathrm{EBP} \beta$ until pre-adipocytes reach the mitotic clonal expansion stage [Tang \& Lane, 2000]. As C/EBP $\beta$ and $\mathrm{C} / \mathrm{EBP} \delta$ increase in expression, $\mathrm{C} / \mathrm{EBP} \beta$ stimulates expression of $\mathrm{PPAR} \gamma$ (peroxisome proliferator-activated receptor $\gamma$ ), which in complex with $\mathrm{RXR} \alpha$ (retinoid $\mathrm{x}$ receptor alpha, a member of the nuclear receptor subfamily 2B1) induces expression of $\mathrm{C} / \mathrm{EBP} \alpha$ but reduces the expression of both $\mathrm{C} / \mathrm{EBP} \beta$ and $\mathrm{C} / \mathrm{EBP} \delta$ [Brun et al., 1996; Tang \& Lane 2012]. In the end, $\mathrm{C} / \mathrm{EBP} \alpha$ and $\mathrm{PPAR} \gamma$ reciprocally regulate one another to ensure that adipocytes are maintained in a differentiated state [Pouols et al., 2010; Tang \& Lane, 2012]. The cells then exit the cell cycle, lose their fibroblast-like appearance, commence accruing cytoplasmic triglyceride, and attain the look and metabolic structures of adipocytes by activating SREBP (sterol regulatory element binding protein 1c, a helix-loop-helix leucine zipper protein which regulates lipogenesis) [Green \& Kehinde, 1974; Student et al., 1980; Tang \& Lane 2012]. Triglyceride accumulation has also been shown to be very closely associated with an organized escalation in expression of the enzymes required for fatty acid and triacylglycerol biosynthesis [Student et al., 1980; MacDougald \& Lane 1995]. Similarly, several regulatory proteins, are also expressed, including insulin receptors [Reed \& Lane, 1980], the insulin responsive glucose transporter GLUT4 [Kaestern et al., 1989; Millar et al., 1999], leptin [Hwang et al., 1997], among others. The adipocyte differentiation cascade is also summarized in Figure 39. 
By increasing the localization of the GLUT4 (a glucose transporter) to the plasma membrane, insulin has been shown to stimulate transport of glucose in adipocytes as well as striated muscle [Bryant et al., 2002]. In the absence of insulin, GLUT4 undergoes endocytosis, where part of the intracellular GLUT4 resides in the common endocytic compartments, whereas the other half is found in a specialized GLUT4 storage compartment which lacks the common endocytic markers [Martin et al., 1996; Zeigerer et al., 2002; Lodhi et al., 2007]. Recently, it has been shown that the two pools of GLUT4 are in equilibrium which is accomplished through uninterrupted cycling and fusion that stimulates intracellular retention of GLUT4 [Karylowski et al., 2004]. Insulin permits escape from the aforementioned cycle, thereby promoting trafficking of GLUT4 positive vesicles to the cell surface [Lodhi et al., 2007]. The stimulation of glucose transport by insulin causes the tyrosine phosphorylation of adaptor proteins (from the insulin receptor substrate family), resulting in localization and activation of both PI3K (phosphatidylinositol 3-kinase) as well as PI3P- (phosphatidylinositol 3-phosphate) dependent processes [Kelly et al., 1992; Kelly \& Ruderman, 1993; Lodhi et al., 2007].

On the other hand, as humans are living longer and as the median age of the global world population is increasing, it should be of no surprise that the frequency of common neurodegenerative disorders (such as Parkinson's and Alzheimer's disease) has also been steadily rising [Sloane et al., 2002; Brookmeyer et al., 2007; Allen et al., 2011], since the greatest risk factor for the aforementioned dementias is age. Hence, there is paramount interest in elucidating the mechanisms behind these debilitating dementias, as a dramatic rise in median age of the world's population will lead to a dramatic increase in the prevalence of these dementias in the upcoming years with the potentially unbearable 
resulting impacts on global healthcare, since there is no known cure for the aforementioned ailments.

Neurotrophins, apart from being indispensable for development of the nervous system, are also key for survival, maintenance and regeneration of adult neurons. Furthermore, fluctuations in expression of neurotrophins have been associated with the pathogenesis of several neurodegenerative disorders (such as Alzheimer's disease), but on the other hand, have also offered a therapeutic answer for others (such as multiple sclerosis) [Kalinowska-Lyszczarz, 2012]. Of particular interest is the nerve growth factor (NGF), which has been shown to mediate learning, memory and other higher brain tasks, as well as to influence survival and differentiation of neurons in rat pheochromocytoma PC12 as well as human neuroepithelioma SK-N-C cells [Huang \& Reichardt, 2001; Segal, 2003; Berry et al., 2012]. It has previously been shown that NGF binds to its receptor TrkA with high affinity and is internalized via receptor mediated endocytosis into signaling endosomes in order to sustain cell signaling and neurite outgrowth [Layer \& Shooter, 1982; Zhang et al., 2000]. Furthermore, it is known that the aforementioned signaling endosomes escape the canonical fusion with late endosomes and subsequent degradation in lysosomes [Liu et al., 2007], possibly by inhibiting activity of Rab5 (a small GTPase that is essential for early endosome fusion and subsequent entry to the degradative endocytic pathway), however biogenesis of signaling endosomes is currently poorly understood.

The Rab superfamily of small GTPases is a key regulator of numerous cellular trafficking processes including the endocytosis of GLUT4 and TrkA vesicles [Dugani and Klip, 2005]. Rabs switch between an active (GTP-bound) and an inactive (GDP- 
bound) form, which is regulated by guanine nucleotide exchange factors (GEFs) that promote exchange of GDP for GTP, and GTPase-activating proteins (GAPs) that speed up the intrinsic GTPase activity of Rabs. Since Rab proteins play a critical role in vesicle trafficking, it may be fair to assume that they also may represent a site of integration for signals from insulin and TrkA receptor as well as signaling endosome biogenesis. In particular, Rab5 is known to promote early endosome fusion and is essential in promoting entry into the late endosome and lysosome degradative pathway [Bucci et al., 1995; Barbieri et al., 1996]. Although extensive research has been centered on both adipogenesis as well as neurogenesis (and subsequently GLUT4 and TrkA trafficking), the specific role of Rab5, the master regulator of endosome biogenesis, in both of the aforementioned processes has yet to be determined. Rabex-5, a well-established GEF for Rab5, catalyzes the exchange of GDP for GTP and in doing so activates Rab5, but also stabilizes it on early endosomes and in doing so recruits other effector proteins to the early endosome in order for specialized trafficking functions to be carried out [Jovic et al., 2010]. Lambright's group has shown that activation of Rab5 is done through the Vps9 catalytic domain and that the highly conserved amino acids D313, P317, Y354 and Y357 are vital in maintaining the full GEF activity of Rabex-5 [Delprato et al., 2004]. Furthermore, Rabex-5 has been shown to inhibit Ras signaling [Colicelli, 2010] via the ubiquitin ligase activity of its $\mathrm{ZnF}$ domain and in doing so shown to be important in establishing organism size, wing vein pattern and eye versus antennal fate in Drosophila [Yan et al., 2010]. Therefore, we were interested in investigating the role of Rabex-5 in differentiation of 3T3-L1 pre-adipocytes into adipocytes as well as in neurite outgrowth of $\mathrm{PC} 12$ cells. 


\subsection{Materials and Methods}

\subsubsection{Cell Culture and Materials}

3T3L1 murine pre-adipocytes cells were grown in DMEM supplemented with $10 \%$ fetal calf serum supplemented with $2 \mathrm{mM}$ L-Glutamine and 1\% pen/strep. PC12 cells were grown in DMEM supplemented with 10\% horse serum and 5\% fetal calf serum and $1 \%$ pen/strep. Rab5, Rabex-5, $\alpha$-Tubulin, C/EBP $\alpha, \operatorname{PPAR} \gamma$ and phospho-EGFR antibodies were from Cell Signaling Technology (Beverly, MA). Rin1 antibody was purchased from Abcam Inc. and total EGFR antibody was from Santa Cruz Biotechnology Inc (Santa Cruz, CA). NGF was purchased from Merk KGaA (Darmstadt, Germany). All HRP labeled secondary antibodies were purchased from Jackson ImmunoResearch Laboratories (West Grove, PA). All other chemicals were purchased from Sigma (St. Louis, MO) unless otherwise noted.

\subsubsection{T3-L1 differentiation protocol}

3T3-L1 pre-adipocytes were seeded in either 6 or 12 well plates at about 2-4 $\times 10^{4}$ cells $/ \mathrm{cm}^{2}$ and induced to differentiate 48 hours post confluent using growth media supplemented with insulin, dexamethasone and IBMX for 48 hours. Cells were then fed growth media supplemented with insulin, with fresh media being added every other day until day 10, when they were either subject to lysing (20 mM Tris- $\mathrm{HCl}$ (pH 7.5), $150 \mathrm{mM}$ $\mathrm{NaCl}$ and $1 \%$ Triton $\mathrm{x}-100$ ), SDS-PAGE and subsequent western blotting or stained with Oil Red-O for lipid quantification as previously described [van Goor et al., 1986]. 


\subsubsection{PC12 differentiation}

PC12 pheochromocytoma cells were seeded in either 6 or 12 well collagen coated plates at about 2-4 x $10^{4}$ cells $/ \mathrm{cm}^{2}$ and grown until $70-80 \%$ confluent with growth media being changed every 48 hours. To induce differentiation growth media was replaced with differentiation media (DMEM supplemented with $1 \%$ Horse serum, $0.5 \%$ calf serum, $1 \%$ pen/strep and 50ng/ml NGF) and changed every 48 hours for 6 days as previously described [Green \& Tischler, 1982; Obin et al., 1999]. Cells were then either fixed in paraformaldehyde for image analysis or subject to SDS-PAGE and subsequent western blotting.

\subsubsection{Western Blot analysis}

To prepare whole cell lysates, cell were washed twice with ice-cold PBS and lysed in ice-cold lysis buffer (20mM Tris- $\mathrm{HCl} \mathrm{pH} 7.5,150 \mathrm{mM} \mathrm{NaCl}, 1 \%$ Triton X-100). The lysates were clarified by centrifugation, and protein concentrations were determined using the BCA Protein Assay Reagent Kit (Pierce). Proteins were resolved by SDSPAGE and transferred to nitrocellulose membranes, which were blocked and probed with the indicated antibodies. To determine relative protein amounts, three representative exposures for each sample were quantified using National Institutes of Health ImageJ software.

\subsubsection{Cell proliferation}

3T3-L1 cells expressing Rabex-5: FL, Rabex-5: Long or Rabex-5: Short splice variants were seeded at $5 \times 10^{5}$ cells in a 12 well dish. Cells were then grown for 48 hours, after which they were treated with MTT reagent for 2 hours and lysed using cell 
lysis buffer containing $10 \% \mathrm{SDS}$ and $0.01 \mathrm{M} \mathrm{HCl}$. Relative proliferation was then quantified by spectrophotometry.

\subsubsection{Cell Imaging and analysis}

Images of fixed cells were obtained using the DeltaVision Elite wide field microscope under differential interference contrast illumination. The number of differentiated PC12 cells was determined visually by counting cells that had at least one neuronal process with a length that was twice the cell body diameter, and expressed it as a percentage of the total cells within that field of view. Neuronal process growth was determined by physically tracing the length of the longest process per cell (using NIH ImageJ software) for all cells in a field of view which had at least one distinguishable neuronal process and for which the entire neuron could be visualized [as previously described by Das et al., 2004].

\subsection{Results}

4.4.1 Rabex-5 splice variants selectively inhibit adipogenesis through activation of Rab5 and ubiquitination of Ras.

Because Over-expression of Rab5 can inhibit differentiation of fat cells, I wanted to assay the effect that an activator of Rab5, Rabex-5, might have on adipogenesis in 3T3L1 cells (add your ref here). First, I compared the expression level of Rabex-5 in control undifferentiated 3T3-L1 fibroblasts to differentiated Day 9 3T3-L1 and noticed that the relative expression of Rabex-5 decreases significantly (Figure 40). I then repeated the experiment but lysed the cells at Days 1, 3, 5, 7 and 9 and observed that as cells undergo differentiation, the expression level of Rabex-5 decreases (Figure 40). 
Because Rabex-5 expression decreases as adipogenesis increases, it was hypothesized that expression of Rabex-5 in 3T3-L1 cells should inhibit the differentiation of 3T3-L1 pre-adipocytes into mature adipocytes. Furthermore, two new splice variants of Rabex-5 (here termed Rabex-5: Short and Rabex-5: Long) in addition to the already published and well characterized splice variant (here termed Rabex-5:FL), I wanted to not only assay whether Rabex-5 inhibits adipogenesis, but also whether these splice variants selectively inhibit adipogenesis. When I quantified adipogenesis by staining the cells with Oil Red$\mathrm{O}$, I observed that although all three splice variants are able to inhibit adipogenesis; Rabex-5: FL and Rabex-5: Long splice variant inhibit adipogenesis about $20-30 \%$ better than Rabex-5 Short splice variant (Figure 41a). Adipogenic cells were lysed to measure adipogenic markers $\mathrm{C} / \mathrm{EBP} \alpha$ and PPAR $\gamma$ and compared to non-adipogenic cells. Rabex-5 inhibited expression of transcription factors $\mathrm{C} / \mathrm{EBP} \alpha$ and PPAR $\gamma$ in comparison to control 3T3-L1 adipocytes (Figure 41).

In order to make sure that the observed inhibitory effect of Rabex-5 was not due to its potential ability to inhibit cellular growth and proliferation, MTT assay showed that the splice variants have no effect on the rate of cell proliferation (Figure 42). Because Rabex5: Short splice variant inhibited adipogenesis less than the other two splice variants and because Rabex-5: Short splice variant is missing two structural domains of the full length splice variant, deletion mutants of Rabex-5 were constructed in order to decipher which domain(s) were contributing to the inhibitory adipogenic effect of Rabex-5. I observed that both the $\mathrm{N}$-terminus and the Vps9 domains significantly inhibited adipogenesis of 3T3-L1 cells, but that the C-terminus had no effect (Figure 43). Because Rabex-5 
interacts with Ras, 3T3-L1 cells expressing Rabex-5: FL exhibited an increase in ubiquitination compared to control fibroblast or Day 10 adipocytes after immunoprecipitation with ubiquitin (Figure 44). On the other hand, because the Vps9 domain of Rabex-5 is the catalytic domain involved in activating Rab5, point mutations in the Vps9 domain in amino acid residues which have been shown to be important in the Rab5-Rabex-5 interaction were generated and could rescue the differentiation of 3T3-L1 cells. The effective residue was Y354 (Figure 43).

\subsubsection{Rabex-5 splice variants selectively inhibit neurite outgrowth in rat PC12 cells}

Because of the inhibitor effect on Rabex-5 splice variants on differentiation of fat cells, the same effect may be true in other cell types, such as neuronal cells. In order to assay differentiation and neurite outgrowth of PC12 cells, between 4-5 x 10 5 cells were grown on glass coverslips that were previously coated with $4 \mu \mathrm{g} / \mathrm{ml}$ Type IV Rat tail collagen, allowed the cells to adhere, and about 2 hours after attachment, changed the media from growth (DMEM supplemented with 10\% horse serum, $5 \%$ fetal calf serum and $1 \% \mathrm{pen} / \mathrm{strep}$ ) to differentiation (DMEM supplemented with $1 \%$ horse serum, $0.5 \%$ calf serum, $1 \%$ pen/strep and 50ng/ml NGF). The media.was replenished every two days and on day 6 fixed the cells with 4\% paraformaldehyde. Images of neuronal processes were taken to measure neurite outgrowth and calculate the percentage of cells that had differentiated. Differeniated cells were those that had a neuronal process that was twice the diameter of the cell body (Figure 45). I observed that Rabex-5: FL and Rabex-5: Long splice variants both inhibited differentiation of PC12 cells (respectively by approximately $25 \%$ and $30 \%$ ), whereas Rabex-5: Short splice variant inhibited differentiation by only 
about $10-15 \%$. Furthermore, PC12 cells expressing the D313A mutation (from the Vps9 domain of Rabex-5: FL) differentiated similarly to control PC12 cells (Figure 46) suggesting to the role of the Vps9 domain in PC12 differentiation.

\subsection{Discussion}

Because it has been previously shown that activation of Rab5 leads to an inhibition in adipogenesis, the effect Rabex-5, a Rab5 GEF, on differentiation of 3T3-L1 preadipocytes was assayed. First 3T3-L1 cell lines expressing Rabex-5 were generated by retroviral infection. Next, cells were grown to confluency and induced to undergo differentiation by supplementing the growth media for the first three days with insulin, dexamethasone and IBMX and then for the next six days only with insulin. Cells were then either lysed and subject to subsequent western blotting (in order to check for adipogenic markers) or fixed and stained with Oil Red-O (in order to quantify the formed liposomes). As expected, in comparison to control 3T3-L1 adipocytes, the 3T3-L1 cells expressing Rabex-5 significantly inhibited adipogenesis (Figures 40 \& 41). In an experiment with differentiating neuronal pheochromocytoma cell line by using NGF, a similar result was obtained: Rabex-5 inhibited neurite outgrowth in PC12 cells (Figure 46).

Furthermore splice variants of Rabex-5 from human placenta (Rabex-5: Short and Rabex-5: Long) in addition to Rabex-5: FL were assayed with differentiating 3T3-L1 preadipocytes; the two splice variants (Rabex-5: FL and Rabex-5: Long) which activate Rab5, also inhibit adipogenesis and neurogenesis, whereas Rabex-5: Short is also able to inhibit adipogenesis and neurogenesis, however significantly less (Figures $41 \& 46$ ). In order to rule out the possibility that the observed inhibitory effect of the selected Rabex-5 
splice variants is merely due to the fact that they may affect the growth and proliferation of these cells, 3T3-L1 cell lines expressing Rabex-5: FL, Rabex-5: Long or Rabex-5: Short splice variants were seeded at $5 \times 10^{5}$ and subject to a cell proliferative MTT assay and it was observed that none of the splice variants affect the rate of cell proliferation (Figure 42).

Because Rabex-5: Short splice variant is missing the terminal 130 amino acids (which comprise the last 5 amino acids of the Vps9 as well as the whole Coiled-Coil and Proline Rich domains), but is still able to inhibit adipogenesis by about $30 \%$, a C-terminal deletion mutant was created in order to assess whether this domain contributes to the antiadipogenic effect of Rabex-5. C-terminus of Rabex-5 did not significantly affect adipogenesis (Figure 43). Additional deletion mutants of Rabex-5 were created in order to better understand both the anti-adipogenic and anti-neurogenic activity of Rabex-5. Hence, we created an N-terminal mutant (which contains the Ras ubiquitination domains), a Vps9 domain (which activates Rab5) from Rabex-5: FL mutant as well as the truncated Vps9 domain from Rabex-5: Short splice variant and observed that although the Vps9 domain (full or truncated) inhibits adipogenesis by about $40-50 \%$ and neurite outgrowth by about 30\%, the N-terminal domain also possesses anti-adipogenic and antineurogenic properties as well (Figures $43 \& 46$ ). Because $\mathrm{ZnF}$ and MIU domains of the $\mathrm{N}$-terminus comprise the Rabex-5 ubiquitin ligase domain and essential in ubiquitination of Ras [Colicelli, 2010], it is plausible to assume that the Rabex-5 may inhibit differentiation of either fat or nerve cells via ubiquitination of Ras and thereby inhibition of Ras signaling. To support this evidence, the immunoprecipitation assay on 3T3-L1 
fibroblast, 3T3-L1 adipocytes and 3T3-L1 adipocytes cells stably expressing Rabex-5 increased ubiquitination of Ras with the Rabex-5 cell line (Figure 44). However, in order to confirm that it was the interaction of Rabex-5 with Rab5 which was causing the majority of adipogenic or neurogenic inhibition, point mutants were generated in the Vps9 domain, at amino acid residues which were previously shown to be key for intact activation of Rab5, those being D313, P317, Y354 and Y357 [Delprato et al., 2004]. The relative differentiation of 3T3-L1 pre-adipocytes as well as PC12 cells was also rescued in these mutants (Figure $43 \& 46$ ), suggesting that the interaction of Rabex-5 with Rab5 is key in controlling the adipogenesis in 3T3-L1 and neurite outgrowth in PC12 cells. In conclusion, expression of Rabex-5 significantly inhibits adipogenesis and neurogenesis because: a) because the $\mathrm{ZnF}$ domain of Rabex-5 ubiquitinates Ras and thereby inhibits Ras signaling and b) more importantly because the GEF domain of Rabex-5 activates Rab5 and in doing so inhibits formation of signaling endosome and in turn promotes the fusion with degradative endosomal trafficking route. 


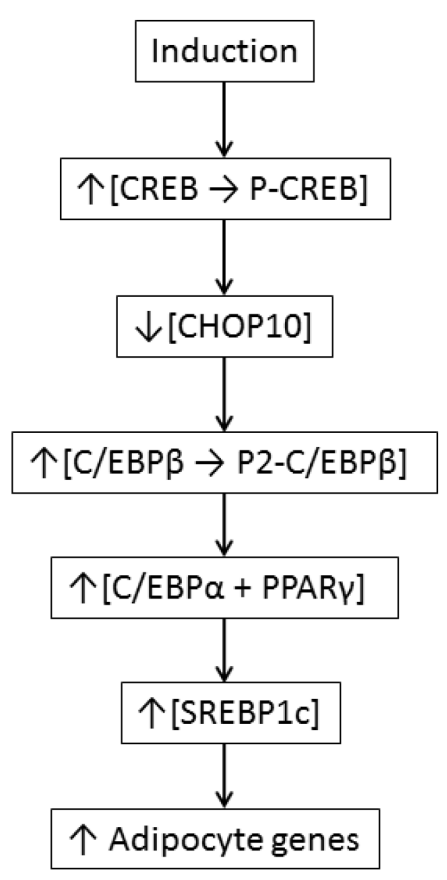

Figure 39. Summary of the adipocyte differentiation cascade. Induction of preadipocyte differentiation is initiated by supplementing the growth media with insulin/IGF, dexamethasone and IBMX, which causes and a phosphorylation of CREB (cAMP response element binding protein) and a decrease in CHOP10 (C/EBP homologous protein 10) allowing the pre-adipocytes to enter mitotic clonal expansion, which is followed by both an increase in C/EBP $\beta$ (CCAAT/enhancer-binding protein beta) as well as the dual phosphorylation of C/EBP $\beta$ first by MAPK and then by GSK3 $\beta$ (glycogen synthase kinase 3 beta). Phosphorylated C/EBP $\beta$ stimulates expression of $\operatorname{PPAR} \gamma$ (peroxisome proliferator-activated receptor $\gamma$ ) which in turn stimulates expression of $\mathrm{C} / \mathrm{EBP} \alpha$ and subsequently reduces expression of $\mathrm{C} / \mathrm{EBP} \beta$. PPAR $\gamma$ and $\mathrm{C} / \mathrm{EBP} \alpha$ then reciprocally regulate one another in order to ensure that adipocytes are maintained in a differentiated state, after which the cells exit the cell cycle, lose their fibroblast-like appearance, commence accruing cytoplasmic triglycerides, and attain the look and metabolic structures of adipocytes by activating SREBP (sterol regulatory element binding protein 1c, a helix-loop-helix leucine zipper protein which regulates lipogenesis) as well as several adipocyte genes (which include insulin receptors, GLUT4 and leptin, among others). 

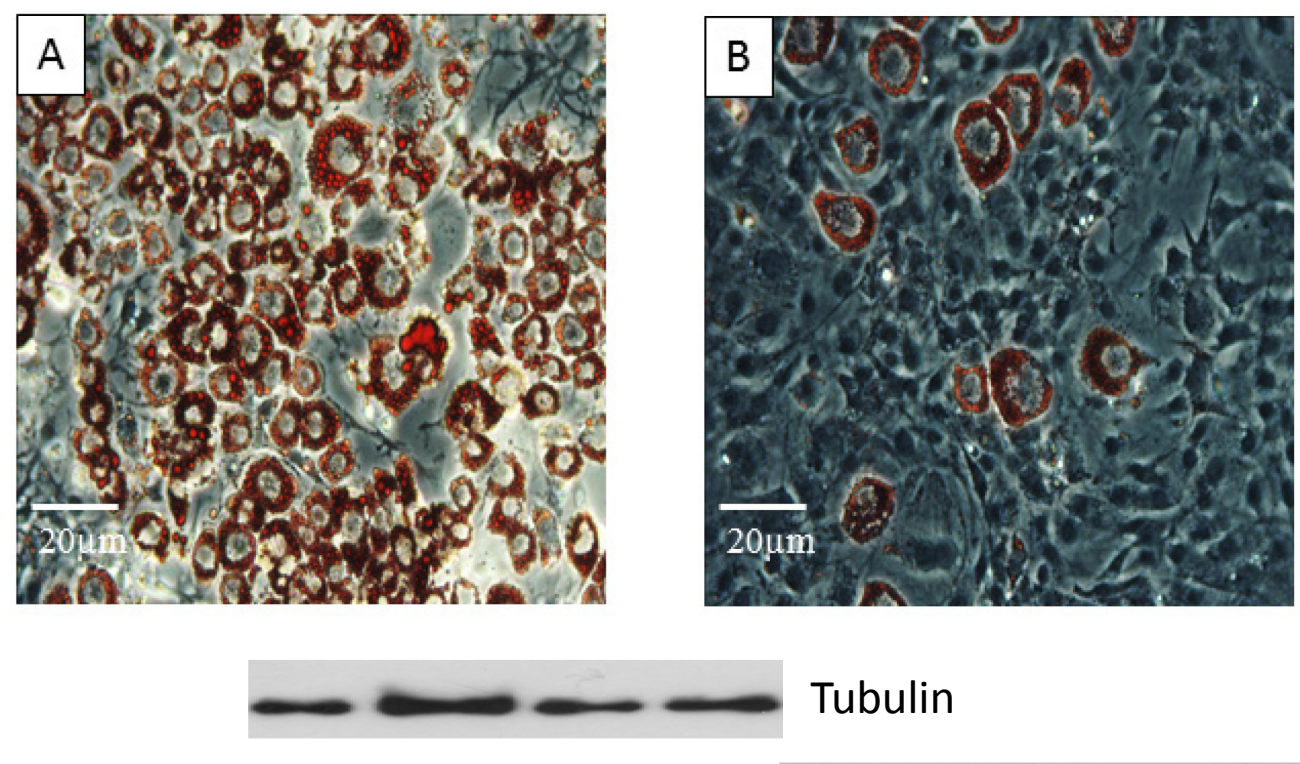

Tubulin

Rabex-5

Fibro Day 3 Day 5 Day 9

Figure 40. Expression of Rabex-5 in 3T3-L1 cells during adipogenesis. I) 3T3-L1 (A) cells or 3T3-L1 Rabex-5 cells (B) were stimulated to differentiate for 9 days after which they were fixed with paraformaldehyde, stained with Oil Red-O (ORO) and imaged using Leica phase contrast microscope, Retiga 1300R camera and QCapture imaging software. II) 3T3-L1 cells were stimulated to differentiate for 9 days and lysed at days 3, 5 and 9, subject to SDS-PAGE and subsequent western blotting with the relative expression level of Rabex-5 compared to undifferentiated 3T3-L1 fibroblasts. 

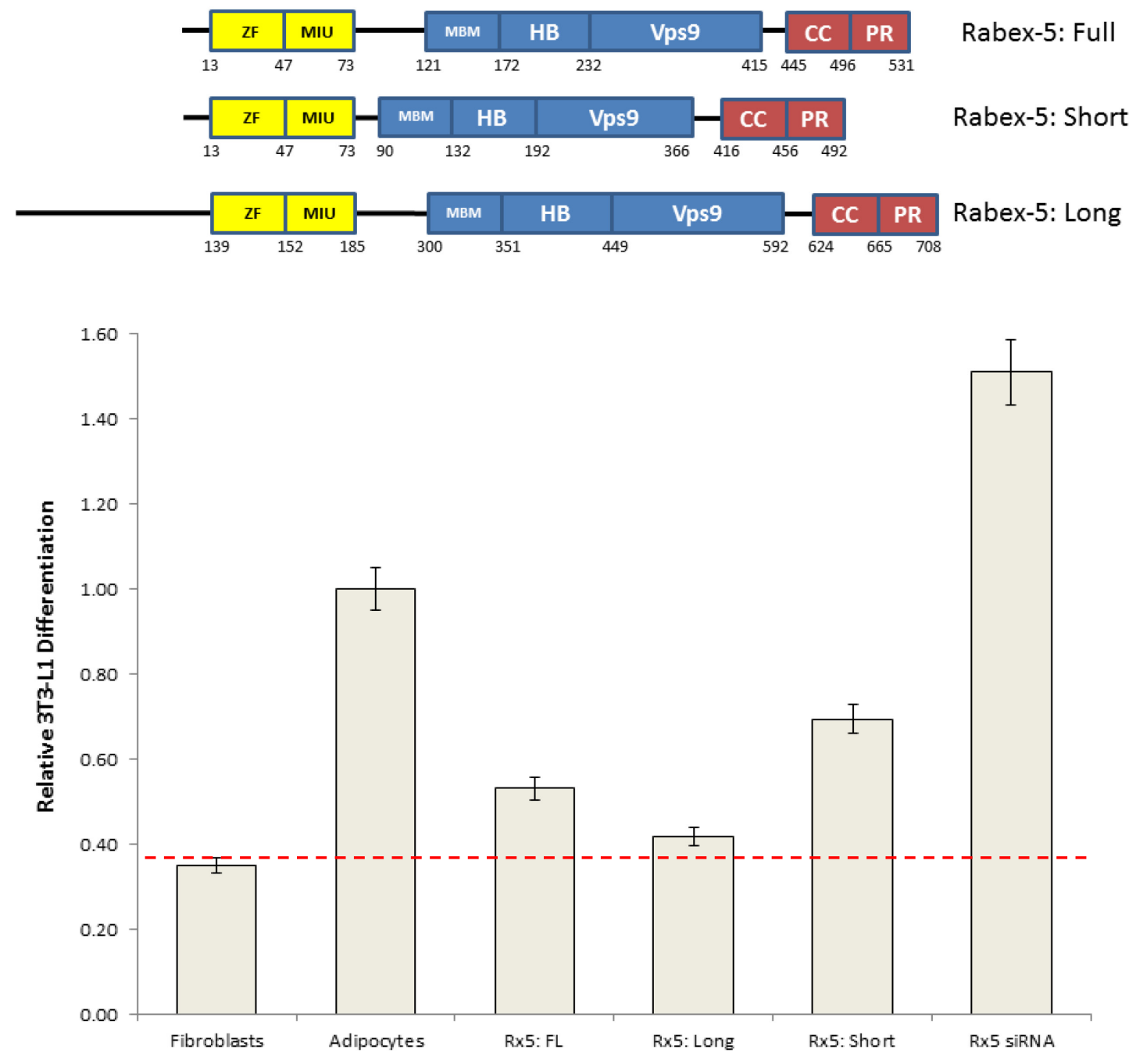

Figure 41. Rabex-5 inhibits differentiation of mouse 3T3L1 pre-adipocytes into adipocytes. Quantification of 3T3-L1 differentiation by Oil Red-O staining. 3T3-L1 cells or 3T3-L1 Rabex-5 cell lines were seeded at $5 \times 10^{5}$ in 12 well plates and grown to confluency and allowed to differentiate for 9 days. Differentiation was induced by supplementing the growth media with insulin, dexamethasone and IBMX at Days 1 and 3 , or only insulin at Days 5,7 and 9, at which point they were fixed in paraformaldehyde, washed with PBS and stained with ORO. ORO was eluted with isopropanol and relative differentiation was quantified by spectrophotometry. 


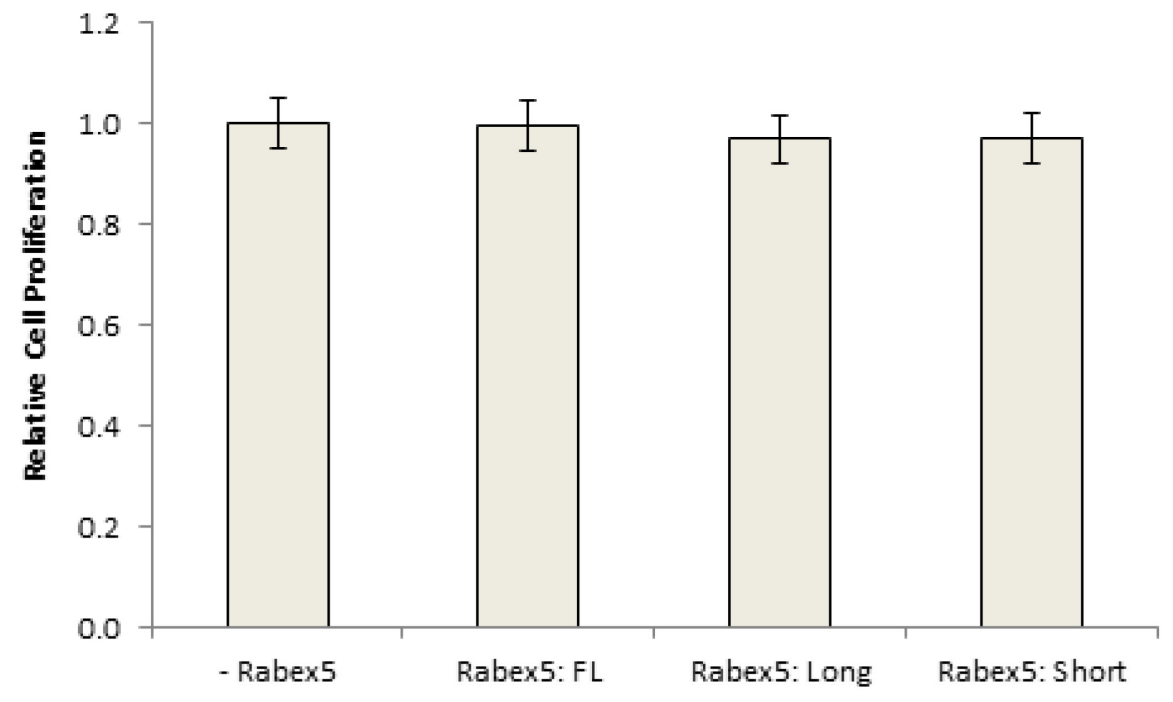

Figure 42. Rabex-5 splice variants do not affect cell proliferation. 3T3-L1 cells expressing the respective Rabex-5 splice variant were seeded onto 12 well plates at $5 \mathrm{x}$ $10^{5}$ cells and allowed to grow for $48 \mathrm{hrs}$ after which they were subject to MTT assay to quantify cell proliferation. The data represent an average of three independent experiments. 
I
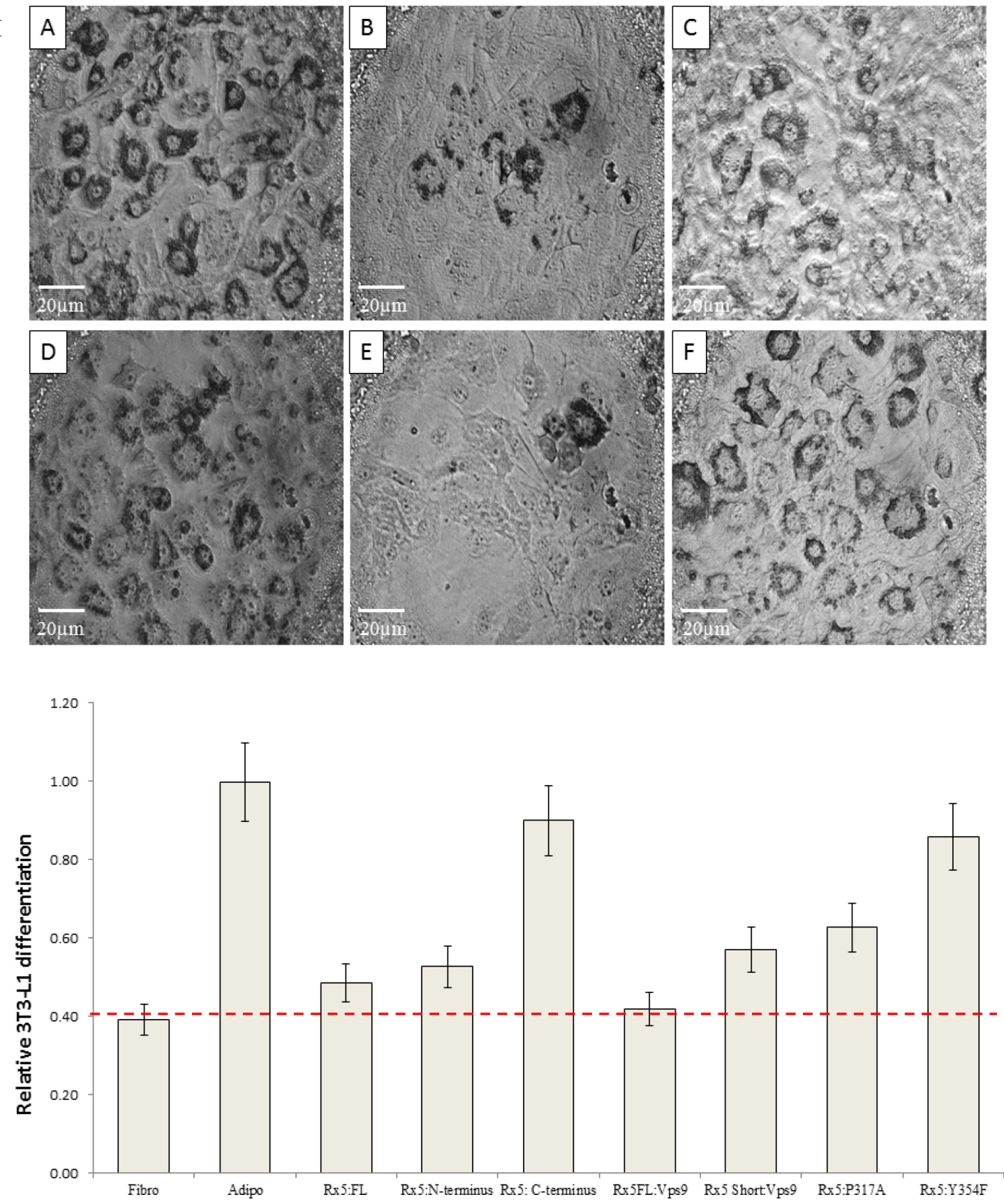

II
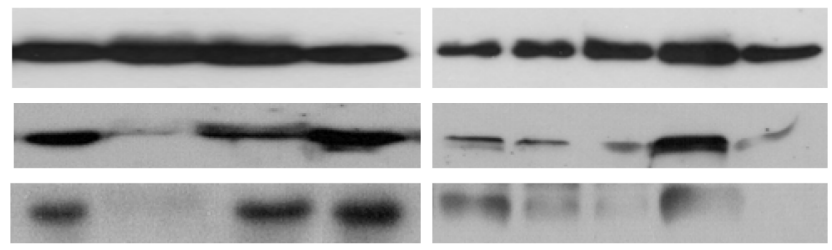

Tubulin

PPAR $-\gamma$

C/EBP- $\alpha$

Rx5:S Rx5: FL Rx5: Rx5: Vps9 Vps9 P317A Y354F

$\begin{array}{ccc}\text { Rx5: } & \text { Rx5: } & \text { Rx5: Adipo Fibro } \\ N & S & F L\end{array}$ 
Figure 43. The Vps9 domain of Rabex-5 is essential in inhibiting differentiation of 3T3-L1 pre-adipocytes into mature adipocytes. I) Quantification of 3T3-L1 differentiation by ORO staining. 3T3-L1 cells or 3T3-L1 Rabex-5 cell lines were seeded at $5 \times 10^{5}$ in 12 well plates and grown to confluency and allowed to differentiate for 9 days. Differentiation was induced by supplementing the growth media with insulin, dexamethasone and IBMX at Days 1 and 3, or only insulin at Days 5, 7 and 9, at which point they were fixed in paraformaldehyde, washed with PBS and stained with ORO. Cells were then washed with $\mathrm{H}_{2} \mathrm{O}$, imaged using phase contrast DeltaVision Elite WideField microscope and SoftWorx imaging software, after which ORO was eluted with isopropanol and relative differentiation quantified by spectrophotometry (A: Control 3T3-L1 adipocytes; B: Rabex-5: FL; C: Rabex-5: Short splice variant; D: Rabex-5: P317A; E: Rabex-5: FL Vps9; F: Rabex-5: Short Vps9). II) 3T3-L1 cells or 3T3-L1 Rabex -5 cell lines were seeded at $5 \times 10^{5}$ in 12 well plates and grown to confluency and allowed to differentiate for 9 days. Differentiation was induced by supplementing the growth media with insulin, dexamethasone and IBMX at Days 1 and 3, or only insulin at Days 5, 7 and 9. At Day 9 cells were lysed and subject to SDS-PAGE and subsequent western blotting in order to check the expression levels of adipogenic markers $\mathrm{C} / \mathrm{EBP} \alpha$ and PPAR $\gamma$ (Fibro- Control Fibroblasts; Adipo- Control adipocytes; FL- Full Length; SShort; N- N terminus). 


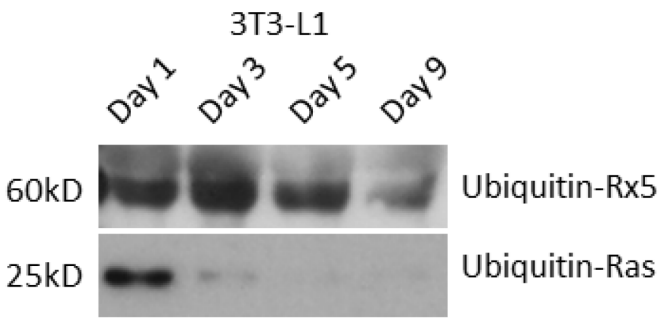

IP: Rabex-5

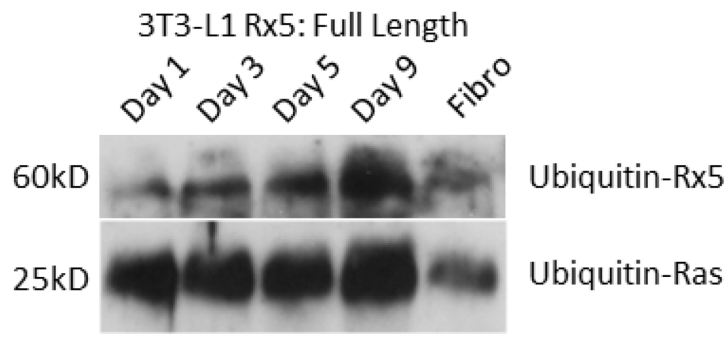

IP: Rabex-5

Figure 44. Rabex-5 increases ubiquitination of Ras in 3T3-L1 cells. 3T3-L1 cells or 3T3-L1 Rabex-5 cell lines were seeded at $5 \times 10^{5}$ in 12 well plates and grown to confluency and allowed to differentiate for 9 days. Differentiation was induced by supplementing the growth media with insulin, dexamethasone and IBMX at Days 1 and 3, or only insulin at Days 5, 7 and 9 after which they were subject to immunoprecipitation using polyclonal rabbit Rabex-5 antibody and IgG beads. Bound protein was eluted from the beads and subject to SDS-PAGE and subsequent western blotting using monoclonal ubiquitin antibody and monoclonal pan-Ras antibody. 

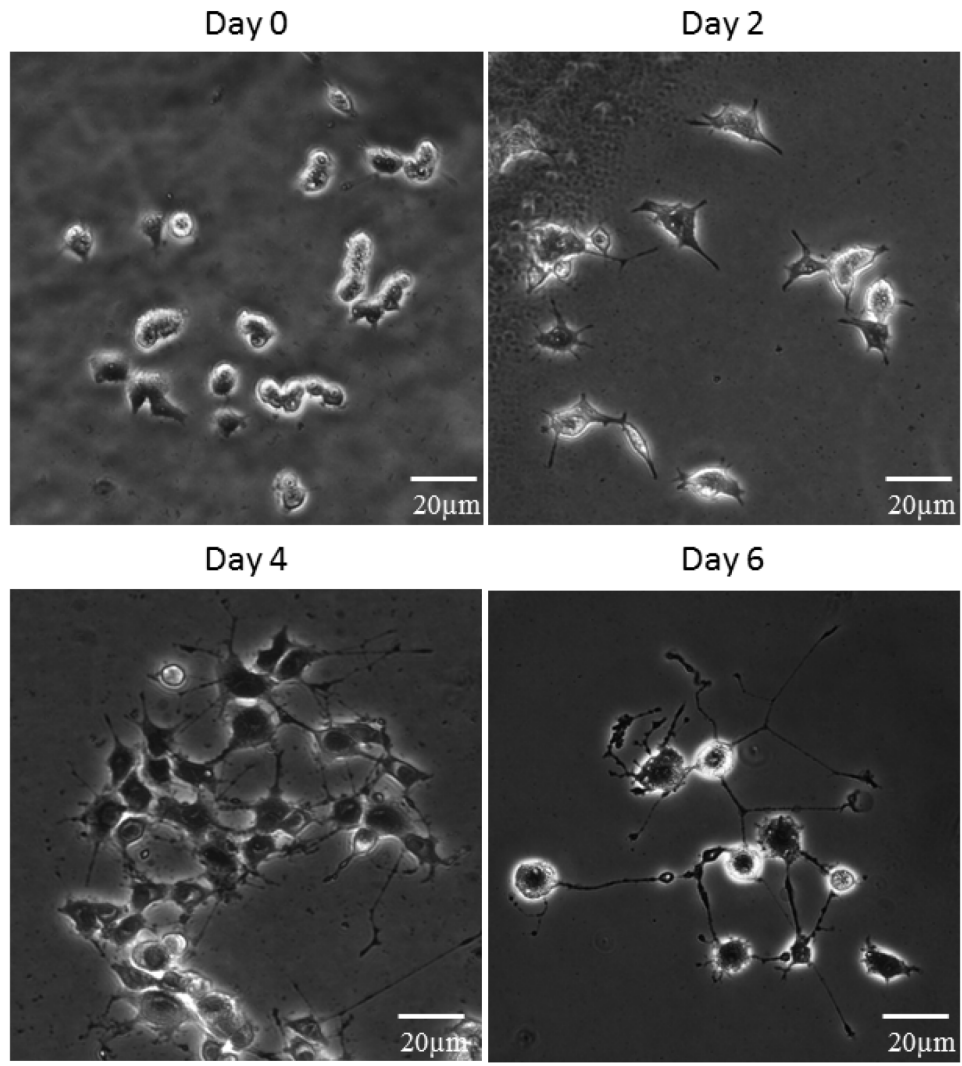

Figure 45. Neurite outgrowth in rat pheochromocytoma PC12 cells. PC12 cells were seeded at $2 \times 10^{4}$ cells $/ \mathrm{cm}^{2}$ onto collagen coated glass coverslips and allowed to differentiate for 6 days. Differentiation was induced by reducing the amount of serum in the media (from $10 \%$ to $1 \%$ ) and supplementing it with $50 \mathrm{ng} / \mathrm{ml} \mathrm{NGF}$, and replenished every 2 days for a total of 6 days. Cells were then fixed with paraformaldehyde either prior to differentiation, after two days of differentiation, after 4 days of differentiation and after 6 days of differentiation, washed with PBS and imaged using DeltaVision Elite wide field microscope under differential interference contrast illumination. 


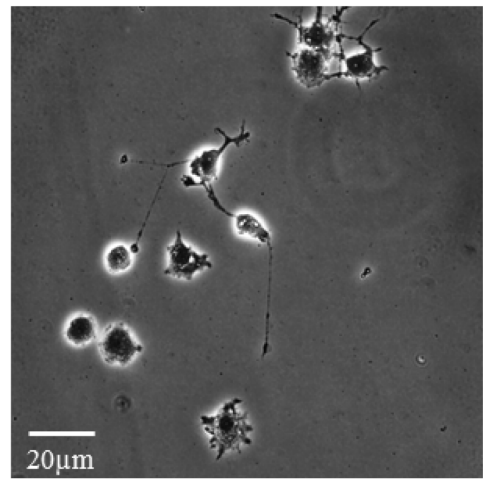

Rabex-5: FL

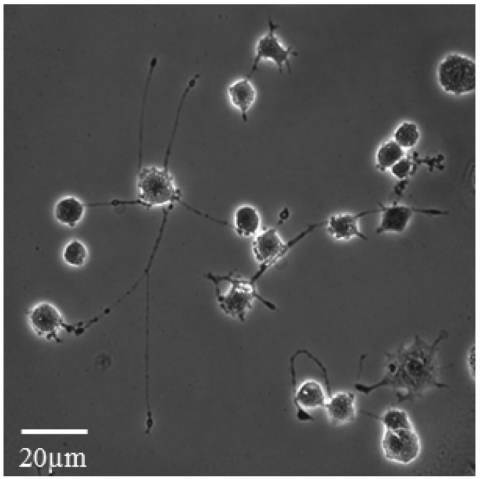

Rabex-5: Long

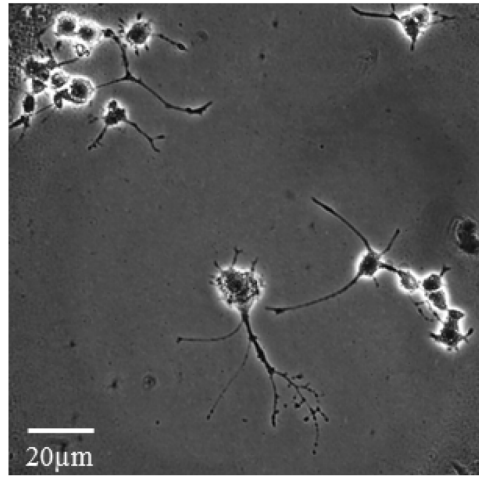

Rabex-5: Short

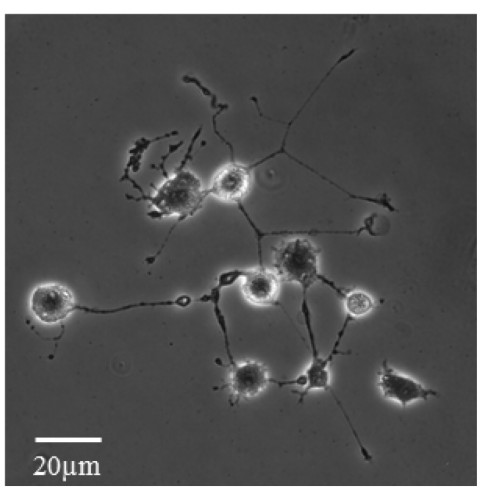

Control PC12

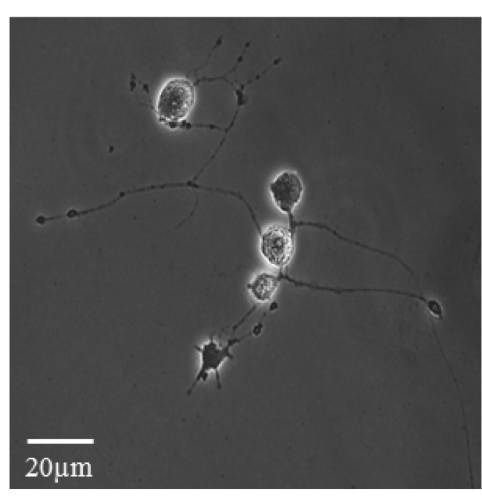

Rabex-5: D313A

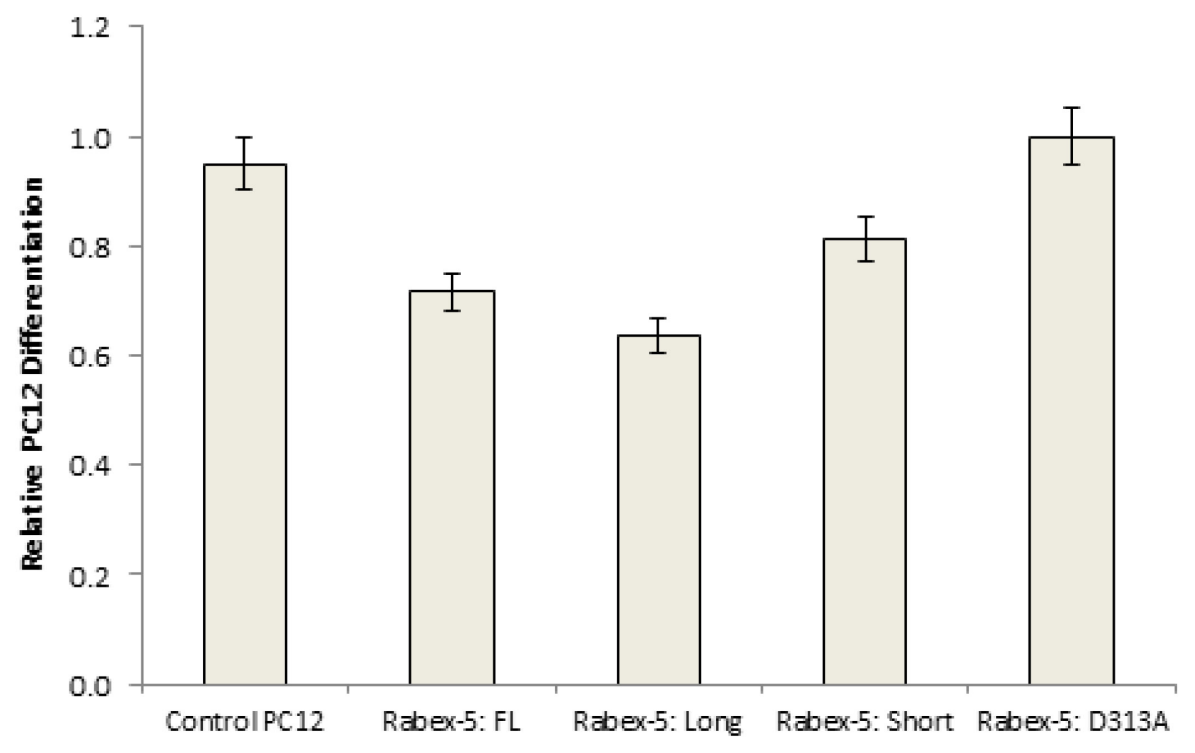


Figure 46. Rabex-5 inhibits neurite outgrowth in rat PC12 cells. PC12 cells and PC12 Rabex-5 cell lines were seeded at $2 \times 10^{4}$ cells $/ \mathrm{cm}^{2}$ on collagen coated glass coverslips and allowed to differentiate for 6 days. Differentiation was induced by reducing the amount of serum in the media (from $10 \%$ to $1 \%$ ) and supplementing it with $50 \mathrm{ng} / \mathrm{ml}$ NGF, and replenished every 2 days for a total of 6 days. Cells were then fixed with paraformaldehyde prior to differentiation, after two days of differentiation, after 4 days of differentiation and after 6 days of differentiation, washed with PBS and imaged using DeltaVision Elite wide field microscope under differential interference contrast illumination. A cell was termed differentiated, if the length of any one of its neuronal processes was twice the diameter of its cell body. 


\subsection{References}

Allen S.J., Watson J.J., Dawbarn D. 2011. The neurotrophins and their role in Alzheimer's disease. Curr. Neuropharmacol. 9(4): 559-573.

Barbieri M.A., Roberts R.L., Mukhopadhyay A., Stahl P.D. 1996. Rab5 regulates the dynamics of early endosome fusion. Biocell 20: 331-338.

Berry A., Bindocci E., Alleva E. 2012. NGF, brain and behavioral plasticity. Neural. Plast. 2012784040.

Brookmeyer R., Johnson E., Zeigler-Graham K., Arrighi H.M. 2007. Forecasting the global burden of Alzheimer's disease. Alzheimers Dement. 3(3): 186-191.

Brun R.P., Kim J.B., Hu E., Altiok S., Spiegelman B.M. 1996. Adipocyte differentiation: a transcriptional regulatory cascade. Curr. Opin. Cell Biol. 8: 826-832.

Bryant N.J., Govers R., James D.E. 2002. Regulated transport of the glucose transporter GLUT4. Nat. Rev. Mol. Cell Biol. 3: 267-277.

Bucci C., Lutcke A., Steele-Mortimer O., Olkkonen V.M., Dupree P., Chiariello M., Bruni C.B., Simons K., Zerial M. 1995. Co-operative regulation of endocytosis by three Rab5 isoforms. FEBS Letters. 366: 65-71.

Coliceli John. 2010. Signal Transduction: RABGEF1 Fingers RAS Ubiquitination. Curr Biol. 20(15): R630-R632.

Das K.P, Freudenrich T.M., Mundy W.R. 2004. Assessment of PC12 cell differentiation and neurite growth: a comparison of morphological and neurochemical measures. Neurotoxicology and Teratology. 26(3): 397-406.

Delprato A., Merithew E., Lambright D.G. 2004. Structure, exchange determinants, and family wide rab specificity of the tandem helical bundle and Vps9 domains of Rabex-5. Cell. 118: 607-617.

Fernyhough M.E., Vierck J.L., Hausman G.J., Mir P.S., Okine E.K., Dodson M.V. 2005. Primary adipocyte culture: adipocyte purification methods may lead to a new understanding of adipose tissue growth and development. Cytotechnology. 46: 163-72.

Flegal K.M., Carroll M.D., Ogden C.L., Curtin L.R. 2010. Prevalence and trends in obesity among US adults, 1999-2008. JAMA. 303: 235-41.

Green H. \& Kehinde O. 1974. Sublines of mouse 3 T3 cells that accumulate lipid. Cell. 1: 113-116.

Green H. \& Meuth M. 1974. An established pre-adipose cell line and its differentiation in culture. Cell. 3(2): 127-133. 
Greene L.A. \& Tischler A.S. 1982. PC12 pheochromocytoma cultures in neurobiological research. Adv. Cell. Neurobiol. 3: 373-414.

Hausman G.J., Dodson M.V., Ajuwon K., Azain M., Barnes K.M., Guan L.L., Jiang Z., Poulos S.P., Sainz R.D., Smith S., Spurlock M., Novakofski J., Fernyhough M.E., Bergen W.G. 2009. The biology and regulation of preadipocytes and adipocytes in meat animals. J. Anim. Sci. 87: 1218-1246.

Huang E.J. \& Reichardt L.F. 2001. Neurotrophins: roles in neuronal development and functions. Annu. Rev. Neurosci. 24: 677-736.

Hwang C.S., Loftus T.M., Mandrup S., Lane M.D. 1997. Adipocyte differentiation and leptin expression. Annu. Rev. Cell Dev. Biol. 13: 231-259.

Jovic M., Sharma M., Rahajeng M., Caplan S. 2010. The early endosome: a busy sorting station for proteins at the crossroads. Histol. Histopathol. 25(1): 99-112.

Kaestner K.H., Christy R.J., McLenithan J.C., Braiterman L.T., Cornelius P., Pekala P.H., Lane M.D. 1989. Sequence, tissue distribution, and differential expression of mRNA for a putative insulin-responsive glucose transporter in mouse 3T3-L1 adipocytes. Proc. Natl. Acad. Sci. USA. 86(9): 3150-3154.

Kalinowska-Lyszczarz A., Pawlak M.A., Michalak S., Losy J. 2012. Cognitive deficit is related to immune-cell beta-NGF in multiple sclerosis patients. J. of the Neurol. Sci. 08 August 2012 (10.1016/j.jns.2012.07.044)

Karylowski O., Zeigerer A., Cohen A., and McGraw T.E. 2004. GLUT4 is retained by an intracellular cycle of vesicle formation and fusion with endosomes. Mol. Biol. Cell 15: $870-882$.

Kelly K.L., Ruderman N.B., Chen K.S. 1992. Phosphatidylinositol-3-kinase in isolated rat adipocytes. J. Biol. Chem. 267: 3423-3428.

Kelly K.L. \& Ruderman, N.B. 1993. Insulin-stimulated phosphatidylinositol 3-kinase. Association with a $185-\mathrm{kDa}$ tyrosine-phosphorylated protein (IRS-1) and localization in a low density membrane vesicle. J. Biol. Chem. 268: 4391-4398.

Kelly T., Yang W., Chen C.S., Reynolds K., He J. 2008. Global burden of obesity in 2005 and projections to 2030. Int. J. Obes. 32:1431-1437.

Layer P.G. \& Shooter E.M. 1982. Binding and degradation of nerve growth factor by PC12 pheochromocytoma cells. J. of Biol. Chem. 258(5): 3012-3018.

Lodhi I.J., Chiang S.H., Chang L., Vollenweider D., Watson R.T., Inoue M., Pessin J.E., Saltiel A.R. 2007. Gapex-5, a Rab31 guanine nucleotide exchange factor that regulates Glut4 trafficking in adipocytes. Cell Metabol. 5: 59-72.

MacDougald O.A. \& Lane M.D. 1995. Transcriptional regulation of gene expression during adipocyte differentiation. Annu. Rev. Biochem. 64: 345-373. 
Martin S., Tellam, J. Livingstone, C. Slot, J.W. Gould, G.W. and James D.E. 1996. The glucose transporter (GLUT-4) and vesicle-associated membrane protein-2 (VAMP-2) are segregated from recycling endosomes in insulin-sensitive cells. J. Cell Biol. 134: 625635.

Mattsson C. \& Olsson T. 2007. Estrogens and glucocorticoid hormones in adipose tissue metabolism. Curr. Med. Chem. 14: 2918-2924.

Millar C.A., Shewan A., Hickson G.R.X. 1999. Differential regulation of secretory compartments containing the insulin-responsive glucose transporter 4 in 3T3-L1 adipocytes. Mol. Biol. Cell. 10: 3675-3688.

Obin M., Mesco E., Gong X., Haas A.L., Joseph J., Taylor A. 1999. Neurite outgrowth in PC12 cells. J. of Biol. Chem. 274: 11789-11795.

Poulos S.P., Dodson M.V. Hausman G.J. 2010. Cell line models for differentiation: preadipocytes and adipocytes. Exper. Biol. and Med. 235: 1185-1193.

Popkin B.M. 2010. Recent dynamics suggest selected countries catching up to US obesity. Am. J. Clin. Nutr. 91: 284S-288S.

Reed B.C. \& Lane M.D. 1980. Expression of insulin receptors during preadipocyte differentiation. Adv. Enzym. Regul. 18: 97-117.

Saxena S., Bucci C., Weis J., Kruttgen A. 2005. The small GTPase Rab5 controls endosomal trafficking and neuritogenic signaling of the nerve growth factor receptor TrkA. J. of Neurosci. 25(47): 10930-10940.

Segal R.A. 2003. Selectivity in neurotrophin signaling: theme and variations. Annu. Rev. Neurosci. 26: 299-330.

Shao Q. \& Chin K.V. 2011. Survey of American food trends and the growing obesity epidemic. Nutr. Res. Pract. 5(3): 253-259.

Student A.K., Hsu R.Y., Lane M.D.. 1980. Induction of fatty acid synthetase synthesis in differentiating 3T3-L1 preadipocytes. J. Biol. Chem. 255: 4745-4750.

Tang Q.Q. \& Lane M.D. 2000. Role of C/EBP homologous protein (CHOP-10) in the programmed activated activation of CCAAT/enhancer-binding protein- $\beta$ during adipogenesis. Proc. Natl. Acad. Sci. USA. 97: 12445-12450.

Tang Q.Q., Gronborg M., Huang H., Kim J.W., Otto T.C., Pandey A., Lane M.D. 2005. Sequential phosphorylation of CCAAT enhancer-binding protein beta by MAPK and glycogen synthase kinase 3 beta is required for adipogenesis. Proc. Natl. Acad. Sci. USA. 102: $9766-9771$.

Tang Q.Q. \& Lane M.D. 2012. Adipogenesis: from stem cell to adipocyte. Annu. Rev. Biochem. 81: 715-736. 
Van Goor H., Gerrits P.O., Grond J. 1986. The application of lipid-soluble stains in plastic-embedded sections. Histochem. 85(3): 251-253.

Yan H., Jahanshahi M., Horvath E.A., Liu H.Y., Pfleger C.M. 2010. Curr Biol. 20: 13781382.

Zeigerer A., Lampson M.A., Karylowski O., Sabatini D.D., Adesnik M., Ren M., McGraw T.E. 2002. GLUT4 retention in adipocytes requires two intracellular insulinregulated transport steps. Mol. Biol. Cell 13: 2421-2435.

Zhang J.W., Klemm D.J., Vinson C., Lane M.D. 2004. Role of CREB in transcriptional regulation of CCAAT/enhancer-binding protein beta gene during adipogenesis. J. Biol. Chem. 279: 4471-4478. 


\section{CHAPTER 5}

CONCLUSION 
The ability of cells to respond to extracellular cues and internalize various materials via different pathways of endocytosis is essential for their normal function. Endocytosis of extracellular molecules has been shown to be involved in numerous cellular activities ranging from growth and division to differentiation and signal transduction. Therefore, understanding the mechanisms controlling endocytosis could prove vital in elucidating numerous debilitating diseases which arise from misregulation of the aforementioned cellular processes ranging from diabetes and Alzheimer's to cancer.

In this dissertation I examined how a small GTPase that is involved in control of early endocytic events is activated, and what effect it may have on differentiation of either fat cells or neuronal cells. Because activation of tyrosine kinases is a key feature in receptor signaling and membrane trafficking processes, and because it has been well established that Rab5 is the master regulator of endocytosis, it was important to understand if there was a correlation between the tyrosine kinase activity of receptor tyrosine kinases and activation or Rab5. By using specific inhibitors of tyrosine kinase activity of EGF- and insulin-receptors (AG1478 for EGF-receptor or AG1024 and HNMPA-(AM) 3 for insulinreceptor), they were able to block fusion of early endosomes and then correlate that to the inhibition of activation of Rab5. Furthermore, it was shown that by using a constitutively active form of Rab5 (Q79L), It was able to rescue endosome fusion upon addition of tyrosine kinase inhibitors both in a cell free system and in intact cells. Hence, the enzymatic activity of tyrosine kinase receptors (either EGF or insulin) is at least in part required for endosome fusion via activation of Rab5. 
Depending on how much ligand is available to the receptor at the cell surface, the receptor-ligand complex can be internalized via number of distinct pathways. These pathways include the canonical clathrin mediated, caveolin mediated, clathrin and caveolin independent but dynamin dependent, clathrin/caveolin/dynamin independent, macropinocytic and phagotycic. Therefore, it was important to understand if Rab5 is responsible for endosome fusion from each of the aforementioned pathways. By using specific inhibitors and siRNA against the major coat proteins or scission proteins involved in each pathway, at least three distinct pathways through which Rab5 can be activated were identified. What's more is that if one of the guanine nucleotide exchange factors for Rab5 (known as Rabex-5) is overexpressed, Rab5 can be activated no matter which pathway is blocked and the need for ligand stimulation of receptor tyrosine kinases is also abolished. The use of three splice variants of Rabex- 5 was used to investigate the ability of each splice variant to activate Rab5. Because all three splice variants are able to activate Rab5 (although at different levels), it is plausible to assume that the three splice variants probably act as redundant activators of Rab5. Because Rab5 is such a key player in early endocytosis, it should be of no surprise that an evolutionarily conserved fail-safe mechanism of activation exists in order to make sure that this crucial protein is tightly regulated.

Next, that if a constitutively active form of Rab5 is expressed in a pre-adipocyte 3T3L1 cell line, differentiation of pre-adipocytes into mature adipocytes is inhibited between $50 \%-60 \%$. So then it was important to understand what role does the aforementioned activator of Rab5, Rabex-5, have in regulating adipogenesis. I have shown that a cell line stably expressing Rabex-5 also inhibits adipogenesis by about 50\%. Furthermore, the 
inhibition of adipogenesis was correlated to activation of Rab5 by showing that expression of Rabex-5 mutants in the Vps9 domain which were previously shown to be essential in activation of Rab5, rescued adipocyte differentiation. The $\mathrm{N}$-terminus of Rabex-5 alone, which does not have the guanine nucleotide exchange capabilities, was also able to inhibit adipogenesis. Since it is well established known that Rabex-5 can ubiquitinate Ras through the $\mathrm{ZnF}$ domain in its $\mathrm{N}$-terminus, the data presented here showsthat in 3T3-L1 cells, Rabex-5 increases ubiquitination of Ras and in doing so interrupts the signaling cascade which would ultimately lead to expression of adipogenic proteins. This was also shown in a cell line that had been commonly employed to study neurogenesis and those cells behaved in a similar manner. Furthermore, in PC12 cells, Rabex-5 inhibits neurite outgrowth by about $30 \%$ and that deletion mutants from the Vps9 domain of Rabex-5 were able to rescue neurogenesis. Therefore, it is concluded that Rabex-5 contributes to inhibition of adipocyte differentiation and neurite outgrowth by promoting degradation of the signaling endosome via: 1) activating Rab5 through the Vps9 domain and promoting fusion with early endosome, and 2) ubiquitination of Ras through the $\mathrm{ZnF}$ domain in the N-terminus and therefore inhibition of the Ras signaling cascade (Figure 47).

Although this dissertation provides better understanding of the interplay between receptor tyrosine kinases and activation of Rab5, a few questions remain to be answered. Although Rab5 is localized endosomes during fluid phase endocytosis and is activated by stimulation with a commonly used fluid phase marker HRP, this activation is much less robust, in comparison to what was observed with either EGF or insulin stimulation in this dissertation. Furthermore, in mammalian cells overexpression of Rabex-5 induces the 
formation of enlarged endosomes, as opposed to either Rin1 or RAP6, even though these guanine nucleotide exchange factors for Rab5 have been shown to be required in some endosome fusion reactions. Therefore, it is plausible that several mechanisms of activation of Rab5 are taking place during endocytosis of receptor tyrosine kinases and subsequent endosome fusion. Although Rabex-5 was shown to inhibit differentiation of fat and neuronal cells through interaction with Rab5, the possibility of its interaction with other effectors remains open, since recently it has been shown that Rab22, an effector of Rabex-5, actually promotes neurite outgrowth in PC12 cells. Better understanding of the interaction of Rab5 guanine nucleotide exchange factors with other members of the Rab5 subfamily (Rab21, Rab22 and Rab31) may prove to be crucial in understanding regulation of various cellular functions that the Rab5 subfamily has been shown to be involved in and provide specific targets for pharmaceutical agents to battle debilitating diseases. 
A

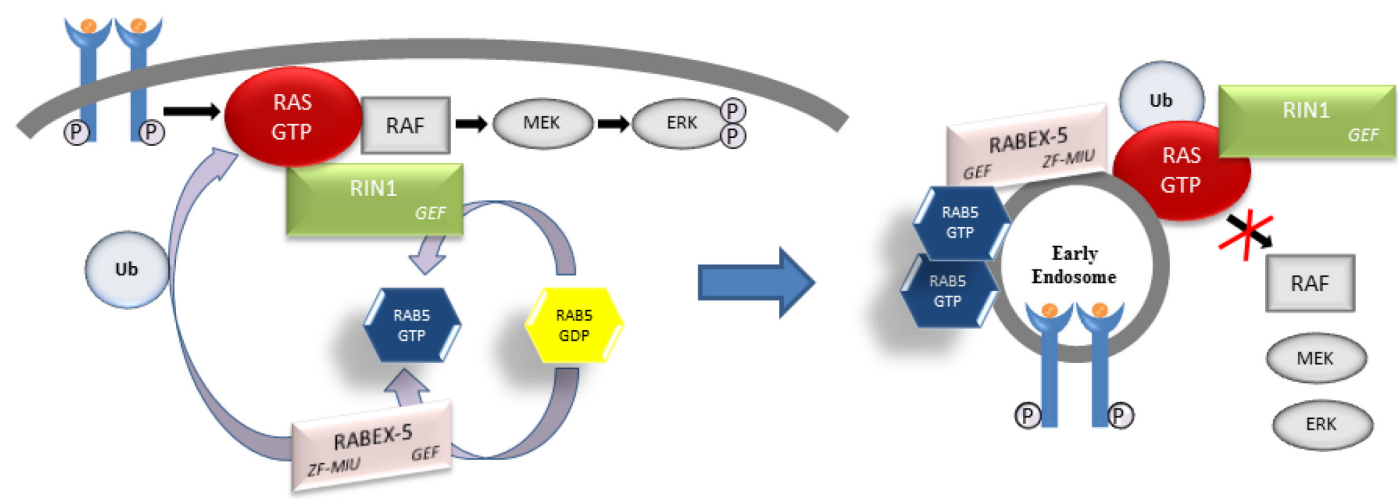

B

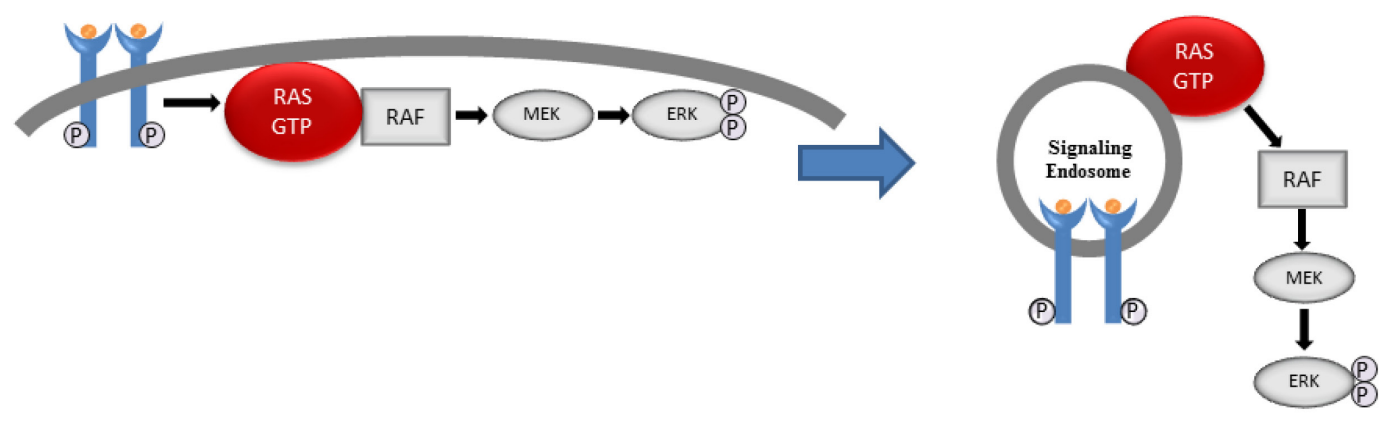

Figure 47. Model for the role of Rabex-5 in differentiation of 3T3-L1 and PC12 cells. Model for Rabex-5 dependent activation of Rab5 and ubiquitination of Ras. A) Binding of ligand to a receptor tyrosine kinase leads to autophosphorylation of the receptors and activation of Ras and the subsequent Ras signalling cascade (Raf-Mek-Erk) which in turn can lead to cellular differentiation, motility, proliferation and survival. On the other hand, Ras also interacts with Rin1 (via the SH2 domain) which in turn activates Rab5 by catalyzing the conversion of GDP for GTP (via the GEF domain). GTP-bound Rab5 then recruits Rabex-5 to the endosome which through its ZnF-MIU domain ubiquitinates Ras and therefore attenuates Ras signalling. The early endosome is then either destined to be recycled back to the cell surface or to sequentially fuse with late endosome and lysosme where the contents of theendosome will be degraded. B) When a cell is undergoing differentiation, there is a marked decrease in Rabex-5 expression, which leads to a decline in Ras ubiquitination, in turn causing an increase in Ras signaling and subsequently the establishment of a signaling endosome (which is void of early endosomal markers) stabilizing Ras signaling and therefore allowing differentiation to continue. 


\section{IVAN JOZIC}

\section{EDUCATION:}

Nova Southeastern University, Ft. Lauderdale, FL

August 2004

Bachelor of Science: Biology

May 2006

summa cum laude

Florida International University, Miami, FL

August 2007

Ph.D. Student: Cell Biology

Present

\section{RESEARCH EXPERIENCE:}

Nova Southeastern University Independent Undergraduate Research

Home Diagnostics Inc. Research Associate

Florida International University Ph.D. Student
Fall 2005

Spring 2006

March 2007

August 2007

August 2007

Present

\section{PRESENTATIONS:}

Ivan Jozic. (2005, March). The role of cyclin-dependent kinase 5 (Cdk5) in Neurodegeneration. Poster presented at the Undergraduate Research Symposium, Nova Southeastern University, Fort Lauderdale, FL.

Ivan Jozic and Vuong, T. (2006, March). The effects of Trolox (Vitamin E analogue) on Gene Expression in $S$. cerevisiae (S288C). In-progress research poster presented at the Undergraduate Research Symposium, Nova Southeastern University, Fort Lauderdale, FL.

Ivan Jozic and Vuong, T. (2006, May). The effects of Trolox (Vitamin E analogue) on Gene Expression in S. cerevisiae (S288C). Poster presented at the Biennial Tri-Beta (National Biological Honors Society) National Convention, Florida Institute of Technology, Melbourne, FL.

Jozic I., Rodriguez Silva M., Barbieri M.A. (2010, December). Structural and Functional Specificity of Rab5 Activating Protein-6 (RAP6). Poster Presentation at the $50^{\text {th }}$ Annual ASCB (American Society for Cell Biology) Conference in Philadelphia, PA. Winner of the MAC Travel Award. 
Rodriguez Silva M., Jozic I., Blanco G., Barbieri M.A. (2010, December). Effect of Tyrosine Kinase Inhibitors on Early Endosome Fusion and Activation of Rab5. Poster Presentation at the $50^{\text {th }}$ Annual ASCB (American Society for Cell Biology) Conference in Philadelphia, PA.

Jozic I., Blanco G., Barbieri, M.A. (2011, January). Effect of Tyrosine Kinase Inhibitors on Early Endosome Fusion and Activation of Rab5. Oral Presentation at $13^{\text {th }}$ Annual FIU Biology Research Symposium, Miami FL.

Jozic I., Stefancin C., Barbieri, M.A. (2011, December). Activation of Rab5 via Diverse Routes of Epidermal Growth Factor Receptor Endocytosis. Poster Presentation at $51^{\text {st }}$ Annual ASCB (American Society for Cell Biology) Conference in Denver, CO. Winner of MAC Travel Award.

Jozic I., Rodriguez Silva M., Barbieri M.A. (2012, December). Selective actiation of Rab5 by distinct endocytic pathways. Poster Presentation at 52 ${ }^{\text {nd }}$ Annual ASCB (American Society for Cell Biology) Conference in San Francisco, CA.

\section{PUBLICATIONS}

Jozic, I. and M.A. Barbieri. 2010. Rab5 and Epidermal Growth Factor Receptor During Endocytosis by Clathrin Dependent and Clathrin Independent Pathways. Current Topics in Biochemical Research. 12(2): 59-67.

Jozic, I., Blanco G., Barbieri, M.A. 2011. Inhibition of Rab5 Activation During Insulin ReceptorMediated Endocytosis. Curr. Cellular Biochem. 1(1): 20-32.

Jozic, I., Saliba S.C., Barbieri, M.A. 2012. Effect of EGF-receptor tyrosine kinase inhibitor on Rab5 function during endocytosis. Arch. Biochem. Biophys. 525(1): 16-24. 